\title{
SPECTROSCOPIC CHARACTERIZATION OF MOLECULAR INTERDIFFUSION AT A POLY(VINYL PYRROLIDONE) / VINYL ESTER INTERFACE
}

\author{
by \\ Christelle Marie Laot

\begin{abstract}
Thesis submitted to the Graduate Faculty of the
Virginia Polytechnic Institute and State University

in partial fulfillment of the requirements for the degree of
\end{abstract}

\section{MASTER OF SCIENCE}

in

Chemical Engineering

Dr. Eva Marand, Chairwoman

\author{
Dr. Richey M. Davis \\ Dr. Hideko T. Oyama \\ Dr. Thomas C. Ward
}

August 25, 1997

Blacksburg, Virginia

Keywords: FTIR-ATR spectroscopy, diffusion, interface, interphase, vinyl ester, poly(vinyl pyrrolidone), plasticization. 


\title{
SPECTROSCOPIC CHARACTERIZATION OF MOLECULAR INTERDIFFUSION AT A POLY(VINYL PYRROLIDONE) / VINYL ESTER INTERFACE
}

\author{
by \\ Christelle Marie Laot \\ Committee Chairwoman: Dr. Eva Marand \\ Chemical Engineering Department
}

(ABSTRACT)

Mechanical properties of (woven carbon fiber / vinyl ester matrix) composites can be greatly improved if the interphase between the reinforcing high-strength low-weight fiber and the thermoset resin is made more compliant. In order to improve the adhesion of the vinyl ester matrix to the carbon fiber, a thermoplastic coating such as poly(vinyl pyrrolidone) (PVP) can be used as an intermediate between the matrix and the fiber. The extent of mutual diffusion at the (sizing material / polymer matrix) interphase plays a critical role in determining the mechanical properties of the composite.

In this research, the molecular interdiffusion across a poly(vinyl pyrrolidone))/vinyl ester monomer (PVP/VE) interface is being investigated by Fourier Transform Infrared Attenuated Total Reflectance (FTIR-ATR) spectroscopy. The ATR method which can be used to characterize the transport phenomena, offers several advantages, such as the ability to monitor the diffusion in situ or to observe chemical reactions. In order to separate the effects of the vinyl ester monomer diffusion and the crosslinking reaction, ATR experiments were carried out at temperatures below the normal curing temperature. Diffusion coefficients were determined by following variations in infrared bands as a function of time, and fitting this data to a Fickian model. The values of the diffusion coefficients calculated were consistent with values found in the literature for diffusion of small molecules in 
polymers. The dependence of diffusion coefficients on temperature followed the Arrhenius equation. Hydrogen bonding interactions were also characterized. The diffusion model used in this study, however, does not seem to be appropriate for the particular (PVP/VE) system. Because the glass transition temperature of the PVP changed as diffusion proceeded, one would expect that the mutual diffusion coefficient did not stay constant. In fact, it was shown that the $\mathrm{T}_{\mathrm{g}}$ can drop by $140^{\circ} \mathrm{C}$ during the diffusion process. A more suitable model of the (PVP/VE) system should take into account plasticization, hydrogen bonding, and especially a concentration dependent diffusion coefficient. Further analysis is therefore needed. 


\section{ACKNOWLEDGMENTS}

I would like to thank the following people for their help and support in conducting this research and for making my American experience enjoyable:

Dr. Eva Marand, my advisor at Virginia Polytechnic Institute and State University, for her support and guidance throughout the past year I have worked with her on my graduate studies. I have especially appreciated her confidence in me, her enthusiasm and her outstanding pedagogical and technical qualities. I am looking forward to furthering my education with her as a PhD student.

Dr. Hideko T. Oyama for helping me with advices on the various research problems I have encountered, and for sending me e-mails, thousands miles away.

Dr. Richey M. Davis and Dr. Thomas C. Ward for serving as other members of my advisory committee.

Dr. J. R. Mahan without whom French students from the Université de Technologie de Compiègne (UTC, France) would never have been able to pursue graduate studies at Virginia Tech.

$\mathrm{M}^{\mathrm{me}}$ Nicole Jaffrin, coordinator of the exchange program between UTC and Virginia Tech, for giving me the opportunity to study in a foreign country and to discover another culture.

The National Science Foundation and Technology Center for High Performance Polymeric Adhesives and Composites at Virginia Tech for its continuous graduate student research assistantship support.

The Ministère de l'Education Nationale, de l'Enseignement Supérieur et de la Recherche, the Ministère de la Défense and the Conseil Général du Finistère for financial support. 
Hui Li, Sean Christian, Kermit Kwan, Slade Gardner and Jay Senkevich for offering valuable technical support.

My labmates for providing an interesting working environment. I am deeply indebted to Dr. Sukhtej S. Dhingra for all the time he dedicated to help me, for all his explanations and advice, for his patience, and above all for his friendship.

All my friends for constantly supporting me and for making me feel at home when times are tough. I am particularly thankful to Ningling Wang, my former classmate and officemate, my present roommate, and simply my best friend here, for her endless encouragements and her culinary support during this past year I have spent in Blacksburg.

My parents and my brothers Philippe and Gérald for their affection, their guidance and their support throughout my studies over the years. None of this would have been possible without them. 


\section{TABLE OF CONTENTS}

ACKNOWLEDGMENTS....................................................................... iv

TABLE OF CONTENTS.......................................................................

LIST OF FIGURES.........................................................................

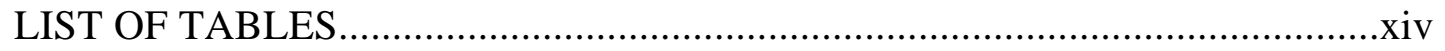

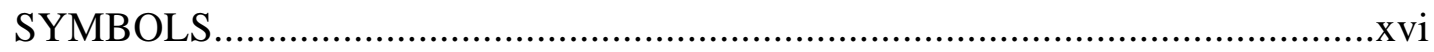

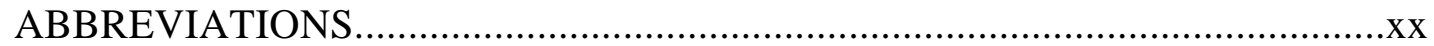

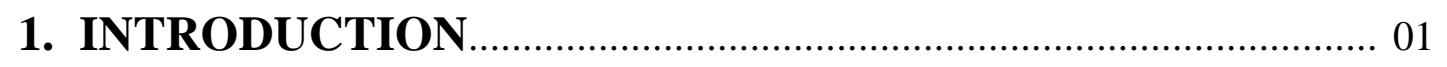

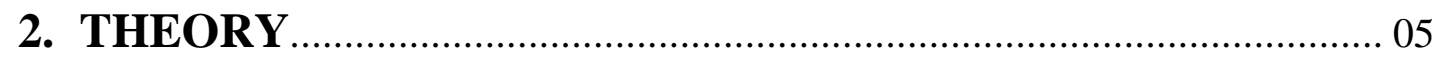

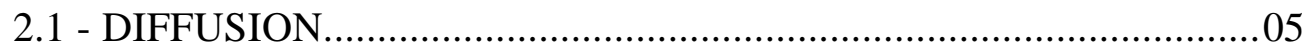

2.1.1 - Mutual Diffusion Versus Self-Diffusion............................. 06

2.1.2 - Experimental Techniques for Measuring Diffusion................06

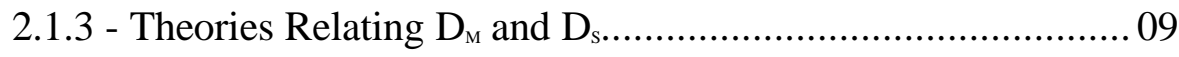

2.1.4 - Comparison Between Theories and Experiments................ 12

2.1.5 - Conclusion.................................................................. 13

2.2 - FTIR-ATR SPECTROSCOPY ................................................. 14

2.2.1 - Infrared Spectroscopy (IR) ......................................... 14

2.2.2 - FTIR Spectroscopy..................................................... 15

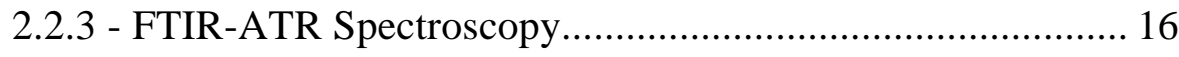




\section{4 - MEASURING DIFFUSION IN POLYMERS}

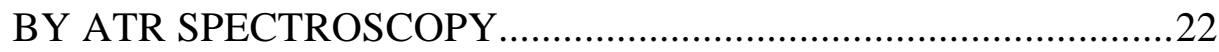

2.4.1 - Expression of the Absorbance............................................... 23

2.4.2 - Diffusion Models..................................................................26

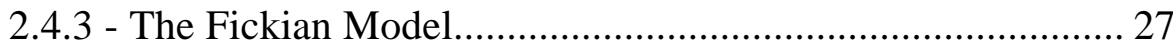

2.4.3.1 - The Interdiffusion System....................................... 27

2.4.3.2 - Fickian Diffusion................................................. 28

2.4.3.3 - Initial and Boundary Conditions................................2 29

2.4.3.4 - Concentration Profile...............................................29

2.4.3.5 - The Diffusion Model..................................................31

2.4.4 - Determination of the Diffusion Coefficients

by ATR Spectroscopy ......................................................... 41

2.4.4.1 - Small Molecule Diffusion into Polymers...................41

2.4.4.2 - Interdiffusion of Polymers........................................43

\section{5 - ADVANTAGES AND LIMITATIONS}

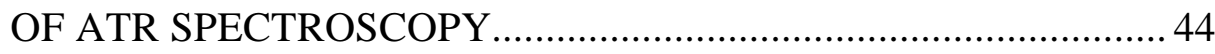

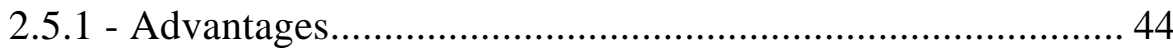

2.5.2 - Limitations..................................................................... 45

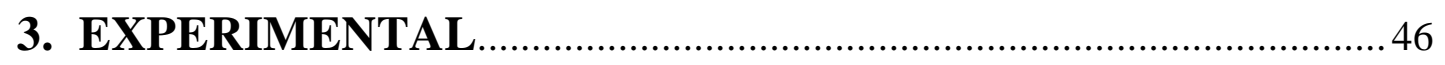

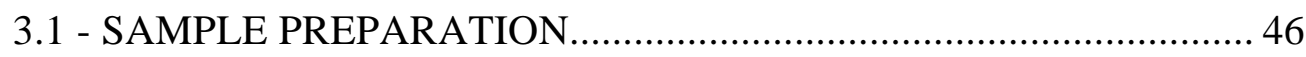

3.1.1 - Choice of the Internal Reflection Element (IRE)

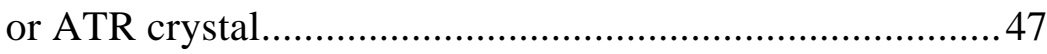

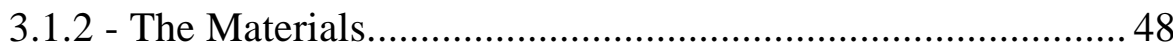

3.1.2.1 - The Poly(Vinyl Pyrrolidone) PVP K90, or PVP..... 48

3.1.2.2 - The Vinyl Ester Monomer, or VE............................51 
3.1.3 - Determination of parameters.............................................. 52

3.1.4 - Preparation of the Sample for the PVP-VE System................53

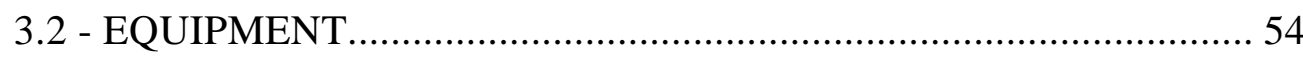

3.2.1 - Temperature Controller......................................................... 55

3.2.2 - Attachment........................................................................ 56

3.2.3 - Infrared Spectrometer...................................................... 56

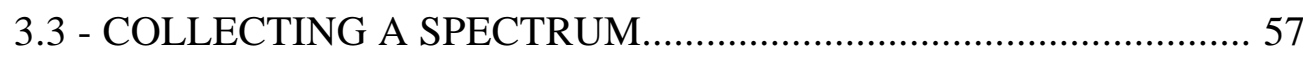

3.3.1 - Spectrometer Set Up.............................................................57

3.3.2 - Spectrum Collection............................................................ 58

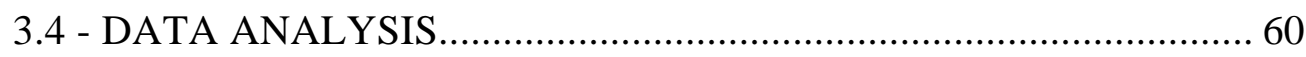

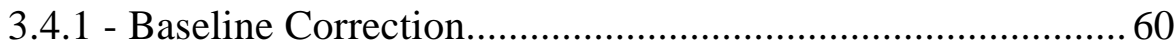

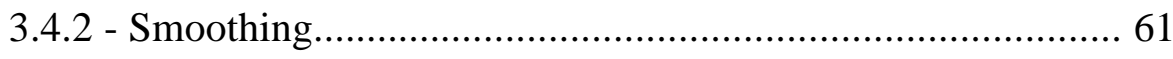

3.4.3 - Spectral Derivative ................................................................. 63

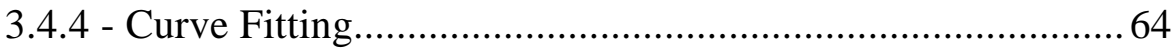

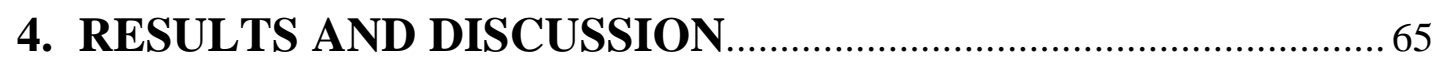

\section{1 - DIFFUSION MEASUREMENTS}

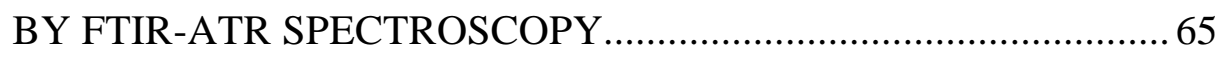

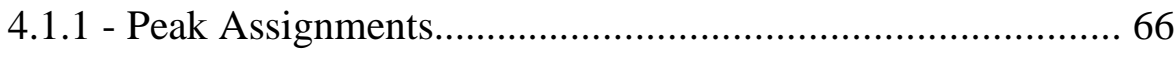

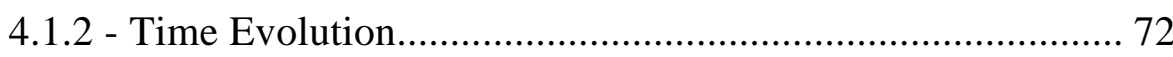

4.1.3 - Determination of the Heights of the Peaks.............................. 74

4.1.4 - Determination of the Diffusion Coefficient...............................76

4.1.5 - Effect of the Temperature.................................................... 80

4.1.6 - Determination of the Activation Energy ................................... 84

4.1.7 - Determination of the Concentration Changes with Time....... 87

4.1.8 - Estimate of the Interphase Thickness....................................... 88 
4.2 - MOLECULAR INTERACTIONS.................................................... 91

4.2.1 - The Hydrogen Bond...............................................................91

4.2.2 - Evidence of Hydrogen Bonding..............................................93

4.2.2.1 - The Carbonyl Region.............................................. 93

4.2.2.2 - Self - Association......................................................95

4.2.2.3 - The Hydroxyl Region.............................................99

4.2.3 - The Equilibrium Constant of Hydrogen Bond Formation...... 100

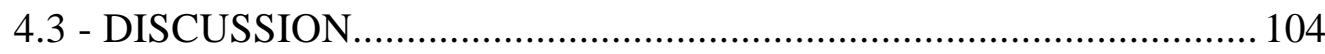

4.3.1 - Discussion of the Errors Involved in the Model..................... 105

4.3.1.1 - Experimental Errors....................................................105

4.3.1.2 - Theoretical Assumptions...........................................110

4.3.2 - How to improve the Diffusion Model?.....................................111

4.3.2.1 - Simultaneous Fickian Model..................................... 114

4.3.2.2 - Plasticization...................................................... 114

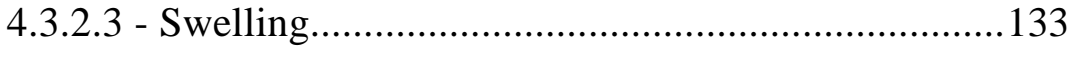

4.3.2.4 - Clustering ............................................................... 133

4.3.2.5 - Concentration Dependent Diffusion Coefficient......134

5. CONCLUSION AND FUTURE WORK ............................................ 136

REFERENCES

APPENDICES

Appendix A: Nomenclature................................................................... 153

Appendix B: Example of Results Obtained by Curvefitting

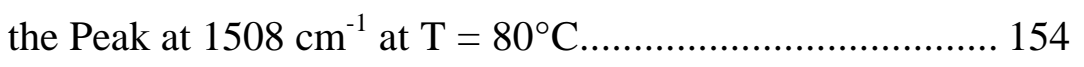


Appendix C: The Fortran Program "diff.for"............................................ 156

Appendix D: The Fortran Program “curvefit.for" .......................................162

Appendix E: The Fortran Program "conc2.for" ....................................... 164

VITA 


\section{LIST OF FIGURES}

Figure 2.1: Schematic of the slow theory of diffusion............................................ 11

Figure 2.2: Schematic of the fast theory of diffusion............................................. 11

Figure 2.3: Schematic representation of total internal reflection.................................. 17

Figure 2.4: Penetration depth, dp, versus angle of incidence $\theta$ at $1507 \mathrm{~cm}^{-1} \ldots \ldots \ldots \ldots \ldots . . . .20$

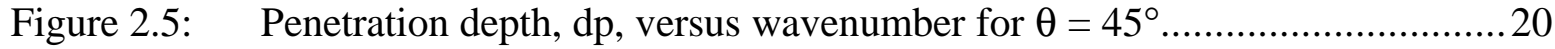

Figure 2.6: Schematic of the evanescent wave near the interface............................... 21

Figure 2.7: Schematic of the interdiffusion system..............................................2

Figure 3.1: $\quad$ Repeat unit of poly(vinyl pyrrolidone)............................................ 50

Figure 3.2: $\quad$ Structure of the vinyl ester monomer............................................. 52

Figure 3.3: Structure of 1,4-Benzoquinone..................................................... 52

Figure 3.4: Schematic of the ATR sample system.............................................54

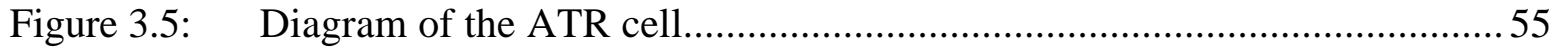

Figure 3.6: Principle of the Seagull TM attachment................................................56

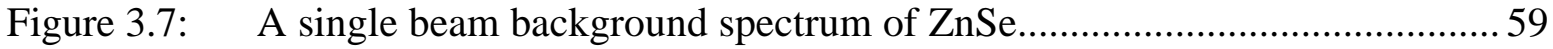

Figure 3.8: Result of baseline correction (top: baseline slope, bottom: after baseline correction)............................ 61

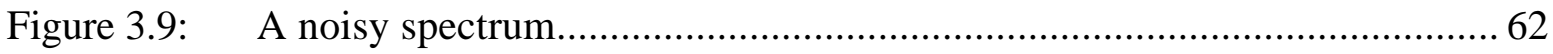

Figure 3.10: The same spectrum after a 9 point Savitsky-Golay smooth........................ 62

Figure 3.11: Example of a second derivative (bottom: original spectrum, top: the second derivative of this spectrum).....63 
Figure 4.1: Infrared spectrum of the poly(vinyl pyrrolidone) or PVP......................... 66

Figure 4.2: Infrared spectrum of the vinyl ester monomer or VE................................67

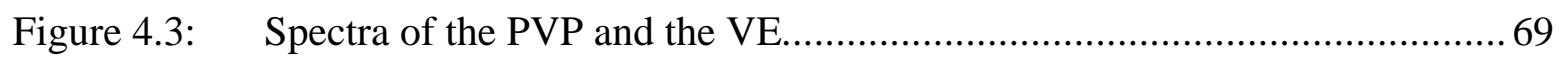

Figure 4.4: Overlapped spectra of the PVP and the VE in the hydroxyl region............. 70

Figure 4.5: Overlapped spectra of the PVP and the VE at low wavenumbers............... 70

Figure 4.6: Overlapped spectra of the PVP and the VE in the region of interest........... 71

Figure 4.7: Time evolution spectra at $\mathrm{T}=100^{\circ} \mathrm{C}$ from $1850 \mathrm{~cm}^{-1}$ to $1350 \mathrm{~cm}^{-1} \ldots \ldots \ldots \ldots .73$

Figure 4.8: Height versus interdiffusion time for the peak at $1507 \mathrm{~cm}^{-1}$ at $\mathrm{T}=80^{\circ} \mathrm{C} \ldots \ldots .75$

Figure 4.9: Height versus interdiffusion time for the peak at $1419 \mathrm{~cm}^{-1}$ at $\mathrm{T}=80^{\circ} \mathrm{C} \ldots \ldots .75$

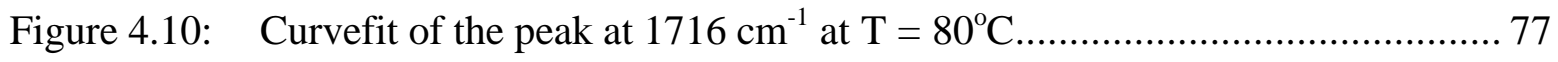

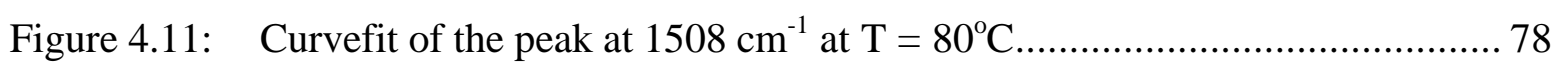

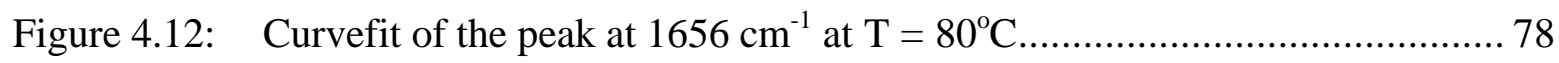

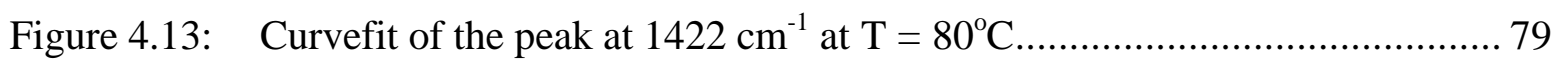

Figure 4.14: Use of the Arrhenius equation for the PVP...................................... 86

Figure 4.15: Use of the Arrhenius equation for the VE...........................................86

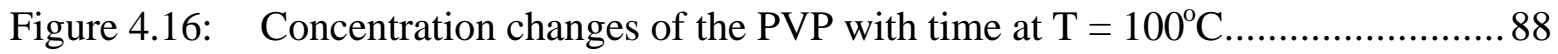

Figure 4.17: Hydrogen bond interaction between the PVP and the VE........................91

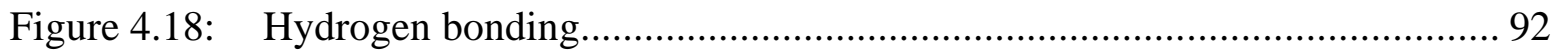

Figure 4.19: Time evolution spectra in the carbonyl region at $\mathrm{T}=100^{\circ} \mathrm{C} \ldots \ldots \ldots \ldots \ldots \ldots \ldots . . . . . . . . . .94$

Figure 4.20: Influence of the temperature $\left({ }^{\circ} \mathrm{C}\right)$ on the pure VE in the carbonyl region..... 96

Figure 4.21: Influence of the temperature $\left({ }^{\circ} \mathrm{C}\right)$ on the pure VE in the hydroxyl region.....96

Figure 4.22: Influence of temperature (from $45^{\circ} \mathrm{C}$ to $120^{\circ} \mathrm{C}$ ) on the pure PVP in the carbonyl region...................................................................99

Figure 4.23: Influence of moisture on the pure PVP in the hydroxyl region.................... 98

Figure 4.24: Influence of moisture on the pure PVP in the carbonyl region....................99

Figure 4.25: Time evolution spectra in the hydroxyl region at $\mathrm{T}=100^{\circ} \mathrm{C} \ldots \ldots \ldots \ldots \ldots \ldots \ldots . \ldots \ldots$

Figure 4.26: Evolution of a 50/50 blend with temperature in the carbonyl region.............103

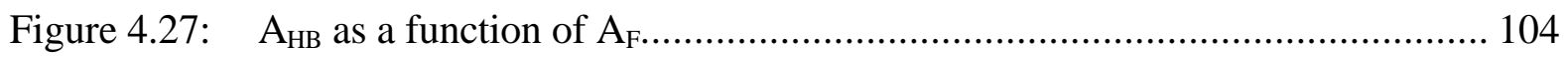

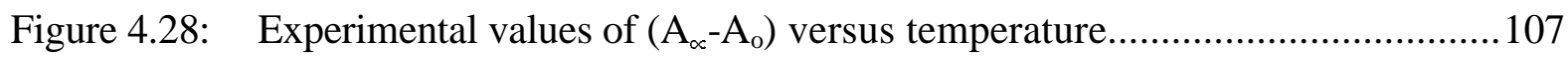




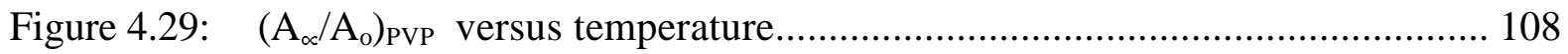

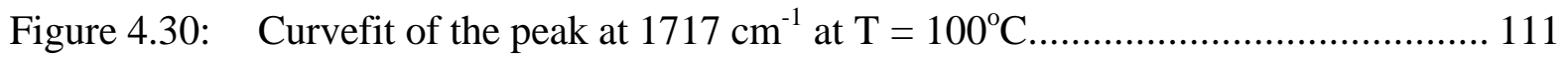

Figure 4.31: Curvefit of the peak at $1507 \mathrm{~cm}^{-1}$ at $\mathrm{T}=100^{\circ} \mathrm{C}$....................................... 112

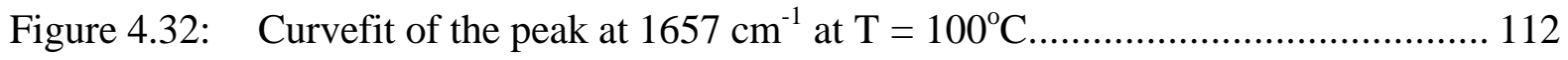

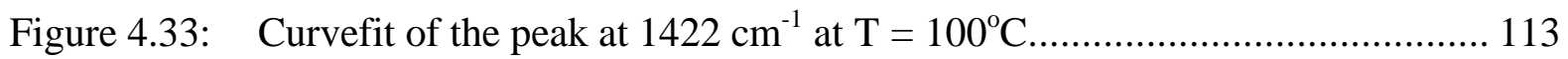

Figure 4.34: Experimental and theoretical $\mathrm{T}_{\mathrm{g}}\left({ }^{\circ} \mathrm{C}\right)$ versus the mass fraction of VE........... 116

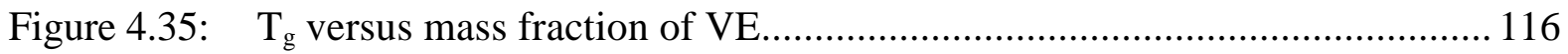

Figure 4.36: Mole fraction of VE carbonyl groups

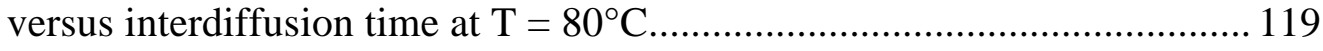

Figure 4.37: Mass fraction of VE versus mole fraction of VE carbonyl groups................. 121

Figure 4.38: Mass fraction of VE versus interdiffusion time at $\mathrm{T}=80^{\circ} \mathrm{C}$........................ 122

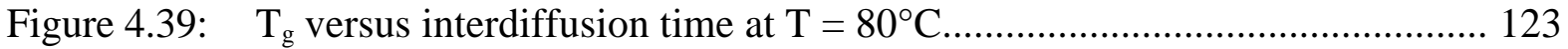

Figure 4.40: Mass fraction of VE versus time for different temperatures......................... 124

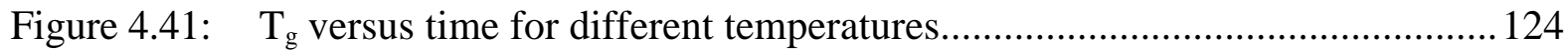

Figure 4.42: Zoom in the early part of Figure 4.30...................................................... 125

Figure 4.43: Curvefit of the $1717 \mathrm{~cm}^{-1}$ peak at $100^{\circ} \mathrm{C}$ cropping the first five data points.. 126

Figure 4.44: Curvefit of the $1717 \mathrm{~cm}^{-1}$ peak at $100^{\circ} \mathrm{C}$ cropping the first six data points... 127

Figure 4.45 Diagram illustrating free volume.......................................................... 128

Figure 4.46: Uptake versus time for the peak at $1507 \mathrm{~cm}^{-1}$ at $\mathrm{T}=100^{\circ} \mathrm{C} \ldots \ldots \ldots \ldots \ldots \ldots \ldots \ldots \ldots . . . . . . . . .130$

Figure 4.47: Uptake versus (time) ${ }^{(1 / 2)}$ for the peak at $1507 \mathrm{~cm}^{-1}$ at $\mathrm{T}=100^{\circ} \mathrm{C} \ldots \ldots \ldots \ldots \ldots \ldots . . .130$

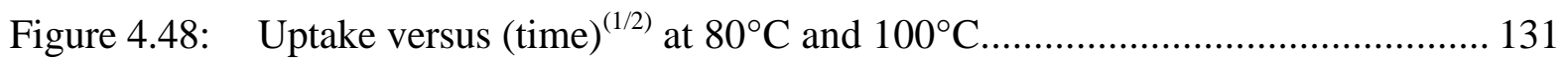

Figure 4.49: Duration time versus temperature............................................................... 131 


\section{LIST OF TABLES}

Table 2.1: Techniques for measuring diffusion at a (polymer/polymer) interface...........07

Table 2.2: $\quad$ Results which agree with the slow theory of diffusion ............................. 12

Table 2.3: $\quad$ Results which agree with the fast theory of diffusion ............................... 12

Table 2.4: $\quad$ Results which disagree with both

the slow and the fast theories of diffusion............................................... 13

Table 2.5: Non-reflection and reflection techniques......................................... 15

Table 2.6: Penetration depth and surface requirements

for some FTIR surface techniques..................................................... 16

Table 3.1: $\quad$ Characteristics of the ZnSe and Si crystals......................................... 47

Table 3.2: $\quad$ Solubility of PVP in various solvents...................................................49

Table 3.3: $\quad$ FTIR spectroscopy sampling parameters.......................................... 58

Table 3.4: $\quad$ FTIR spectroscopy scanning conditions............................................. 59

Table 4.1: $\quad$ Peak assignments of the poly(vinyl pyrrolidone) (PVP)........................... 68

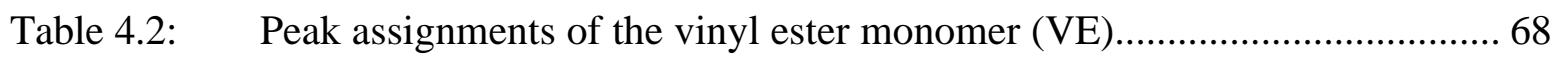

Table 4.3: $\quad$ Assignments of the chosen characteristic bands....................................... 72

Table 4.4: Penetration depths of the peaks of interest........................................ 73

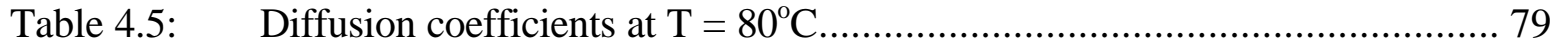

Table 4.6: Diffusion coefficients at different temperatures...................................... 81

Table 4.7: $\quad$ Ratio of diffusion coefficients based on different spectral bands.................. 82 
Table 4.8: Average diffusion coefficients at different temperatures............................83

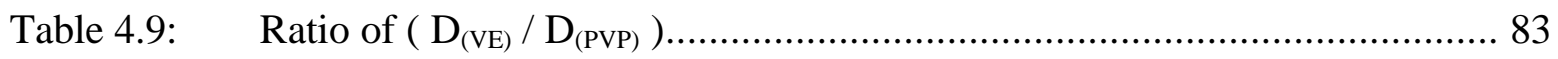

Table 4.10: Activation energies and preexponential factors....................................... 85

Table 4.11: Frequency shifts upon hydrogen bond formation.................................... 95

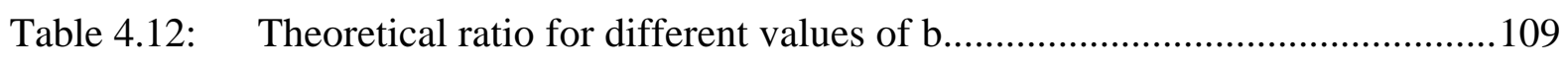

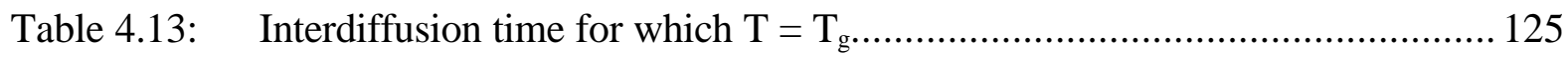




\section{$\underline{\text { SYMBOLS }}$}

a total thickness of the system

a Mark-Houwink constant

$\mathbf{a}^{\mathbf{3}} \quad$ volume per monomer

A absorbance

A(t) absorbance at any time

$\mathbf{A}_{\mathbf{F}}$ area of the band associated to the free carbonyl groups

$\mathbf{A}_{\mathbf{H B}}$ area of the band associated to the hydrogen bonded carbonyl groups

A initial absorbance

$\mathbf{A}_{\mu} \quad$ equilibrium absorbance at infinite time

b thickness of the polymer

C concentration

$\mathbf{C}(\mathbf{z}, \mathbf{t})$ concentration profile of the PVP

$\mathbf{C}_{\mathbf{0}}$ initial concentration of the PVP

$\mathbf{C}_{\mathbf{m}}(\mathbf{z}, \mathbf{t})$ concentration profile of the $\mathrm{VE}$

$\mathbf{C}_{\mathbf{m} 0}$ initial concentration of the VE

d interfacial thickness

dp penetration depth

D diffusion coefficient

$\mathbf{D}_{(\mathbf{P V P})}$ mutual diffusion coefficient of the PVP

$\mathbf{D}_{\text {(VE) }}$ mutual diffusion coefficient of the VE

$\mathbf{D}_{\mathbf{M}} \quad$ mutual diffusion coefficient

$\mathbf{D}_{\mathbf{M F}}$ interdiffusion coefficient for the fast theory

$\mathbf{D}_{\mathrm{MS}}$ interdiffusion coefficient for the slow theory

$\mathbf{D}_{\mathbf{S}} \quad$ self-diffusion coefficient 


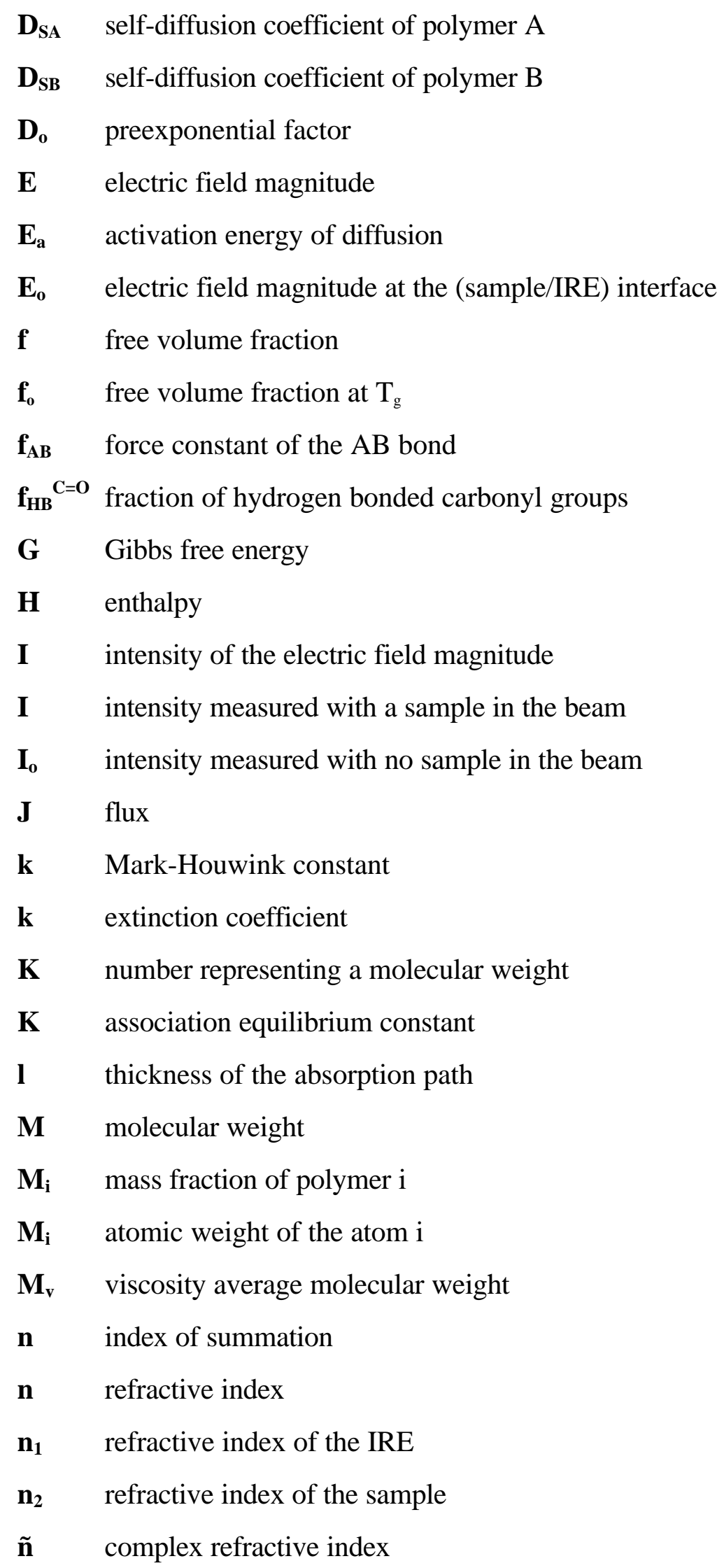




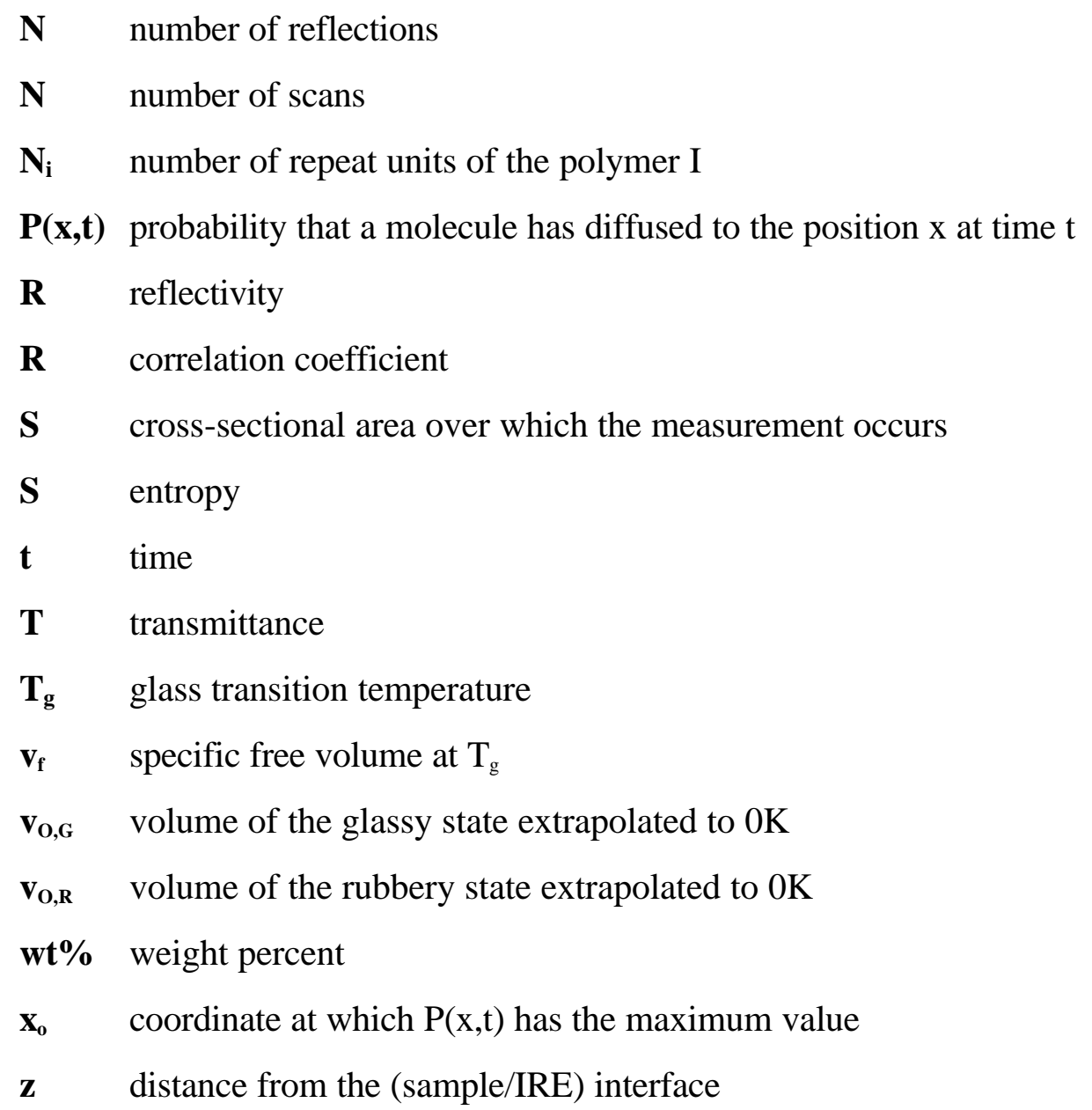




\section{Greek symbols}

$\alpha \quad$ characteristic absorptivity or oscillator strength

$\alpha \quad$ ratio of the absorptivities of the hydrogen bonded band to the one of the free band

$\alpha_{\mathrm{f}} \quad$ expansion coefficient of the free volume

$\boldsymbol{\alpha}_{\mathbf{G}} \quad$ thermal expansion coefficient in the glassy state

$\boldsymbol{\alpha}_{\mathbf{R}} \quad$ thermal expansion coefficient in the rubbery state

$\chi \quad$ Flory-Huggins interaction parameter

$\chi^{2} \quad$ reduced chi-squared parameter

$\varepsilon \quad$ absorptivity

$\boldsymbol{\varepsilon}_{\mathbf{F}} \quad$ absorptivity for the free band

$\boldsymbol{\varepsilon}_{\mathbf{H B}}$ absorptivity for the hydrogen bonded band

$\phi \quad$ volume fraction of polymer A

$\boldsymbol{\gamma}$ frequency $\left(\mathrm{Hz}\right.$ or s$\left.{ }^{-1}\right)$

$\eta_{\text {rel }}$ relative viscosity

[ๆ] intrinsic viscosity

$\lambda \quad$ wavelength $(\mu \mathrm{m})$

$\mu \quad$ chemical potential

$\mu_{\mathrm{A}} \quad$ chemical potential of polymer A

$\mu_{\mathbf{B}} \quad$ chemical potential of polymer B

$\theta \quad$ angle of incidence

$\theta_{c} \quad$ critical angle of incidence

$\sigma \quad$ wavenumber $\left(\mathrm{cm}^{-1}\right)$ 


\section{ABBREVIATIONS}

ASTM American Society for Testing and Materials

ATR Attenuated total reflectance

DTGS Deuterated triglycine sulfate

DSC Differential scanning calorimetry

EMP Electron microprobe analysis

FRES Forward recoil spectrometry

FTIR Fourier transform infrared

IR Infrared

IRE Internal reflection element

IRS Internal reflection spectroscopy

$\mathrm{KBr} \quad$ Potassium bromide

MCT Mercury-cadmium-telluride

NSF National Science Foundation

PDMS Poly(dimethyl siloxane)

PEMS Poly(ethyl methyl siloxane)

PI Polyisoprene

PMMA Poly(methyl methacrylate)

PMMS Poly(phenyl methyl siloxane)

PS Polystyrene

PVME Poly(vinyl methyl ether)

PVP Poly(vinyl pyrrolidone)

PVC Poly(vinyl chloride)

RBS Rutherford backscattering spectrometry

SBR Styrene-butadiene random copolymer 
$\mathbf{S i}$

SIMS

SNR

UTC

VE

VER

ZnSe
Silicon

Secondary ion mass spectrometry

Signal-to-noise ratio

Université de Technologie de Compiègne

Vinyl ester monomer

Vinyl ester resin (VE + 30\% styrene)

Zinc selenide 


\section{CHAPTER 1}

\section{INTRODUCTION}

Polymer composites play an increasingly important role in today's technology. Carbon fiber reinforced composites, composed of carbon fiber and vinyl ester matrix, show poor mechanical properties, which arise as a result of poor adhesion between the fiber and the matrix [1]. Mechanical properties of (woven carbon fiber/thermoset resin) composites can be greatly improved if the interphase between the reinforcing high-strength low-weight fiber and the matrix is made more compliant. In order to improve the adhesion of the vinyl ester matrix to the carbon fiber, a thermoplastic coating such as poly(vinyl pyrrolidone) (PVP) can be used as an intermediate sizing material between the matrix and the fiber [2]. The sizing material has to be compatible with the carbon fiber surface and miscible with the vinyl ester matrix in order to form a particular interphase region [3, 4]. After contact is

established between the PVP and the vinyl ester matrix, adhesion takes place by interdiffusion across the interface [5]. The extent of mutual diffusion at the (sizing material/polymer matrix) interphase plays a critical role in determining the mechanical properties of the composite. The incorporation of PVP coated carbon films in highly crosslinked thermosets improves the toughness, without compromising modulus, strength and chemical resistance [6].

The widespread use of PVP and vinyl ester materials underscores the importance of diffusion of PVP and vinyl ester from both a commercial and a technical perspective. Vinyl ester resins are inexpensive and their properties are superior to those of epoxy resins or unsaturated polyester resins [7] because of low costs and ease of processing. Vinyl ester resins are typically formulated for resin transfer molding [8]. Vinyl ester monomers diluted with styrene are mainly used in thermoset polymer matrix composites $[3,7,9]$. The vinyl 
ester monomer forms a three-dimensional network with the styrene, when crosslinked via free radical copolymerization [10] by opening of the double bonds, $\mathrm{C}=\mathrm{C}$, leading to an addition reaction with no formation of by-products. Vinyl ester monomers diluted with styrene can be fully cured at low temperature very rapidly [11]. Composites based on such materials are being employed mainly in aerospace and structural applications. In addition, thanks to the advantages of resin transfer molding, they also tend to find applications in ground transportation and infrastructure $[12,13]$. An advantage of the PVP polymers is that they are water-soluble and non-toxic, therefore interesting for environmental reasons. Carbon fibers are usually coated with PVP in amounts from 0.5 to 2 wt\% [14].

The particular (PVP/vinyl ester) system has already been studied by a number of researchers. Riffle et al. [15] focused their attention on designing the polymeric interface, employing a vinyl ester resin (VER), Derakane 441-400, commercialized by Dow Chemical Co. This resin consisted of a vinyl ester monomer (VE) diluted with $30 \mathrm{wt} \%$ of styrene in order to reduce the room temperature viscosity. Indeed, the VE does not flow well at room temperature. It is necessary to add a diluent in order for the resin to penetrate well between fibers. Styrene is a good diluent since it is miscible with the VE and is relatively cheap. A cure temperature of $150^{\circ} \mathrm{C}$ was set by the investigators. Some studies have also been done by Oyama et al. [16] focusing on the interface between VER and PVP using electron microprobe analysis (EMP) [17, 18]. Two regions with different diffusion coefficients were observed in the interphase, attributed to the change in solubility at the interface. The diffusion coefficient was estimated to be roughly equal to $10^{-7} \mathrm{~cm}^{2} \cdot \mathrm{s}^{-1}$ at $150^{\circ} \mathrm{C}$. Different (VE-styrene) compositions were studied and it was found that the interfacial thickness increased dramatically with the amount of styrene content.

Although very valuable, the EMP analysis method presents some limitations. Pretreatment of the sample is necessary and experiments have to be done in a vacuum atmosphere at low temperature in order to reduce mass loss. The error involved with the technique is quite important since the sample can be damaged during preparation and 
examination. Another easier technique needs to be used to confirm the results obtained by EMP analysis, and also to verify the existence of a discontinuity in the interphase.

Attenuated Total Reflectance (ATR) spectroscopy has been used to measure interdiffusion for a number of polymer systems $[19,20,21,22,23,24,25,26]$. This technique is attractive for several reasons. It is easy, simple and nondestructive, and the penetration depth ranges between 0.1 to $10 \mu \mathrm{m}$. Any concentration change within the penetration depth due to diffusion of one polymer in another one can be observed in situ. The diffusion of each polymer can then be followed independently, provided that the compounds have some infrared distinguishable absorption bands. It is possible to work in a broad range of temperatures (below and above the $\mathrm{T}_{\mathrm{g}}$ ) and with a wide variety of interface combinations (amorphous, crystalline, thermoplastic, thermoset polymers, different molecular weights, etc). The ATR method offers the advantage of measuring the diffusion coefficients over a wide range of values: $10^{-5}$ to $10^{-16} \mathrm{~cm}^{2} \cdot \mathrm{s}^{-1}$, as well as characterizing chemical interactions occuring within the polymeric system.

The objective of this project is to show that it is experimentally possible to measure the diffusion coefficients across a polymeric interface by Fourier Transform Infrared Attenuated Total Reflectance (FTIR-ATR) spectroscopy. The molecular interdiffusion across a poly(vinyl pyrrolidone)/vinyl ester monomer (PVP/VE) interface is being investigated in this research using ATR spectroscopy. The effect of styrene composition on the value of the diffusion coefficient has not been investigated in this research since it was not the primary objective. In order to separate the effects of the vinyl ester monomer diffusion and the crosslinking reaction, ATR experiments were carried out at temperatures below the normal curing temperature. From the value of the diffusion coefficient, one is able to predict the interphase composition profile, and hence the mechanical properties of the composite. 
This thesis is organized as follows. A background on diffusion and on the principles and the use of FTIR-ATR spectroscopy to measure interdiffusion of polymers is being provided in Chapter 2. The experimental part is discussed in Chapter 3, while the results are presented in Chapter 4. 


\section{CHAPTER 2}

\section{THEORY}

This chapter serves as a review of the pertinent literature describing the estimation of diffusion coefficients associated with a polymeric interface employing Fourier Transform Infrared Attenuated Total Reflectance (FTIR-ATR) spectroscopy.

The first section gives an introduction to the topic of diffusion and discusses the possible experimental techniques for measuring diffusion at a (polymer/polymer) interface. The second section provides a short background on infrared spectroscopy. The third section discusses the optical principles of ATR spectroscopy. The fourth section focuses on the diffusion models developed for this particular technique and refers to the work published in the literature. The final section summarizes the advantages and limitations of ATR spectroscopy.

\section{1 - DIFFUSION}

In many applications, such as those involving electronics or aerospace materials, polymers are often in contact with another material. After contact is established between two polymers, adhesion takes place by interdiffusion of the polymers across the interface [5]. The interface represents the thin plane section between the two components. In comparison, an interphase is defined as the region of finite thickness where mechanical and physical gradients occur. The interphase between two polymers is typically developed, as one polymer component diffuses into the other and vice versa. The subject of polymerpolymer interfaces is of interest because of its importance in adhesion, welding, polymer blends, crack healing, latex film formation, and other applications [27, 28]. 
After a brief definition of mutual diffusion coefficient, $D_{M}$, and self-diffusion coefficient, $\mathrm{D}_{\mathrm{S}}$, the following section presents the experimental techniques used to measure diffusion at a (polymer/polymer) interface. The section concludes with an overview of the theories relating $\mathrm{D}_{\mathrm{M}}$ and $\mathrm{D}_{\mathrm{S}}$.

\subsection{1 - MUTUAL DIFFUSION VERSUS SELF-DIFFUSION}

The mean diffusion coefficient of the binary polymeric system $(\mathrm{A} / \mathrm{B})$ consists of three diffusion coefficients: the mutual diffusion coefficient, $D_{M}$, and the two, selfdiffusion coefficients, $\mathrm{D}_{\mathrm{SA}}$, and $\mathrm{D}_{\mathrm{SB}}$, associated with the Brownian motions. The mutual diffusion coefficient, $\mathrm{D}_{\mathrm{M}}$, also called interdiffusion or collective diffusion coefficient, measures the change in concentration of a species from its average concentration with time. $\mathrm{D}_{\mathrm{M}}$ is the diffusion coefficient which has to be taken into account in the Fick's law of diffusion. The mutual diffusion coefficient of $\mathrm{A}$ in $\mathrm{B}$, and the mutual diffusion coefficient of $\mathrm{B}$ in A are considered to be identical $[29,30,31]$. The self-diffusion coefficient, $\mathrm{D}_{\mathrm{S}}$, measures the center of mass mean-square displacement of one polymer chain in the material with time. Three kinds of motions can be distinguished for $\mathrm{D}_{\mathrm{S}}$ : internal, rotational, and translational motions. As the volume fraction of $A$ approaches $1, D_{M}=D_{S B}$, and as the volume fraction of $A$ approaches zero, $D_{M}=D_{S A}$. $D_{S}$ is well-understood [28], contrary to $\mathrm{D}_{\mathrm{M}}$. It is well-established, for instance, that $\mathrm{D}_{\mathrm{S}}$ is proportional to the inverse of the square of the molecular weight for all polymers above their entanglement molecular weight as predicted by the reptation theory, and to the inverse of the molecular weight for all polymers below their entanglement molecular weight as predicted by the Rouse theory.

\subsection{2 - EXPERIMENTAL TECHNIQUES FOR MEASURING DIFFUSION}

The past fifteen years have seen the development of several techniques used to study diffusion at (polymer/polymer) interfaces [28, 30, 32]. Different experimental techniques are used to measure $\mathrm{D}_{\mathrm{M}}$ and/or $\mathrm{D}_{\mathrm{S}}$. Those techniques are summarized in Table 2.1 . 
In order to measure $D_{S}$, chains have to be labeled and this labeling may modify the value of the diffusion coefficient.

Table 2.1: Techniques for measuring diffusion at a (polymer/polymer) interface

\begin{tabular}{|c|c|c|}
\hline Techniques & $\begin{array}{l}\mathrm{D}_{\mathrm{M}} \text { and/or } \\
\mathrm{D}_{\mathrm{S}}\end{array}$ & $\begin{array}{l}\text { Spatial resolution, } \\
\text { Range for D }\left(\mathrm{cm}^{2} / \mathrm{s}\right) \text {, Comments }\end{array}$ \\
\hline $\begin{array}{l}\text { scanning infrared microscopy } \\
\text { infrared microdensitometry }[33,34,35,36] \\
\text { scanning infrared microscopy }[37,38]\end{array}$ & $\begin{array}{l}\mathrm{D}_{\mathrm{M}} \\
\mathrm{D}_{\mathrm{M}}\end{array}$ & $\begin{array}{l}100 \mu \mathrm{m}, 10^{-5} \text { to } 10^{-10} \\
10 \mu \mathrm{m}\end{array}$ \\
\hline $\begin{array}{l}\text { electron microscopy } \\
\text { scanning electron microscopy with energy- } \\
\text { dispersive x-ray spectrometry (SEM/EDS) } \\
{[39,40,41]} \\
\text { transmission electron microscopy (TEM) } \\
\text { [42] } \\
\text { TEM with electron energy-loss spectroscopy } \\
\text { (EELS) [43] } \\
\text { TEM with EDS and EELS }[44,45]\end{array}$ & $\begin{array}{c}\mathrm{D}_{\mathrm{M}} \\
\mathrm{D}_{\mathrm{M}} \\
\mathrm{D}_{\mathrm{M}} \\
\mathrm{D}_{\mathrm{M}}\end{array}$ & $\begin{array}{l}3 \mu \mathrm{m} \text {, destructive technique } \\
50 \mu \mathrm{m}\end{array}$ \\
\hline $\begin{array}{l}\text { light scattering } \\
\text { optical Schlieren technique [46] } \\
\text { spectroscopic ellipsometry [47, 48] } \\
\text { dynamic light scattering [49] }\end{array}$ & $\begin{array}{l}D_{M} \\
D_{M} \\
D_{M}\end{array}$ & $\begin{array}{l}10^{-6} \text { to } 10^{-12} \\
5 \mathrm{~nm} \\
10^{-8}\end{array}$ \\
\hline $\begin{array}{l}\text { neutron scattering } \\
\text { small-angle neutron scattering (SANS) } \\
\quad[63,64,65,66] \\
\text { neutron reflection spectroscopy (NRS) } \\
\quad[47,48,67,68]\end{array}$ & $\begin{array}{l}\mathrm{D}_{\mathrm{S}} \\
\mathrm{D}_{\mathrm{M}}\end{array}$ & $\begin{array}{l}1 \mathrm{~nm}, 10^{-20} \\
1 \mathrm{~nm}\end{array}$ \\
\hline
\end{tabular}


Table 2.1 continued

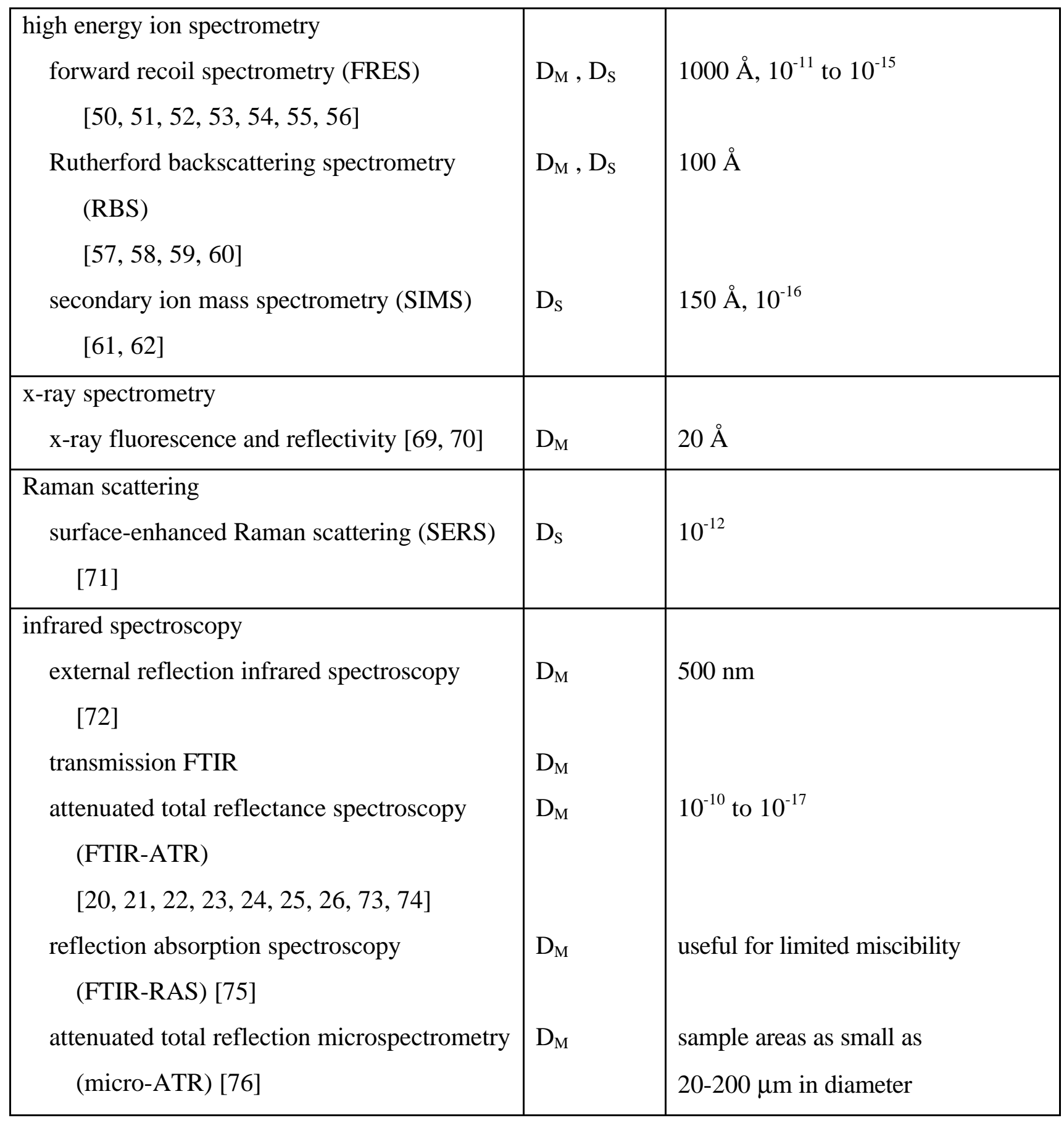


Table 2.1 continued

\begin{tabular}{|c|c|c|}
\hline other methods & & \\
\hline radioactive tracers $[77,78]$ & $\mathrm{D}_{\mathrm{M}}, \mathrm{D}_{\mathrm{S}}$ & \\
\hline $\begin{array}{l}\text { NMR pulsed field-gradient technique } \\
\quad[79,80,81,82,83,84,85]\end{array}$ & $\mathrm{D}_{\mathrm{S}}$ & $10^{-9}$ to $10^{-10}$ \\
\hline $\begin{array}{l}\text { forced Rayleigh scattering from holographic } \\
\text { gratings }[86,87,88]\end{array}$ & $\mathrm{D}_{\mathrm{M}}, \mathrm{D}_{\mathrm{S}}$ & 1 to $100 \mu \mathrm{m}$, from $10^{-5}$ to $10^{-17}$ \\
\hline $\begin{array}{l}\text { fluorescence redistribution after pattern } \\
\text { photobleaching }[89,90,91]\end{array}$ & $\mathrm{D}_{\mathrm{S}}$ & $10^{-12}$ \\
\hline photon correlation spectroscopy $[83,92]$ & $\mathrm{D}_{\mathrm{M}}$ & \\
\hline donor-acceptor fuorescence method [93] & $\mathrm{D}_{\mathrm{M}}, \mathrm{D}_{\mathrm{S}}$ & $50 \AA$ \\
\hline small-angle x-ray scattering (SAXS) [94] & $\mathrm{D}_{\mathrm{M}}$ & \\
\hline electron microprobe analysis [95] & $\mathrm{D}_{\mathrm{M}}$ & $1 \mu \mathrm{m}$ \\
\hline nuclear reaction analysis (NRA) [96] & $\mathrm{D}_{\mathrm{M}}$ & $15 \mathrm{~nm}$ \\
\hline ellipsometry $[97,98]$ & $\mathrm{D}_{\mathrm{M}}$ & \\
\hline
\end{tabular}

\subsection{3 - THEORIES RELATING $D_{M}$ AND $D_{S}$}

If a simple relationship between $D_{M}$ and $D_{S}$ can be found for a binary mixture of two compatible polymers $\mathrm{A}$ and $\mathrm{B}$, it will be possible to predict the temperature and composition dependence of $\mathrm{D}_{\mathrm{M}}$, since $\mathrm{D}_{\mathrm{S}}$ can be measured easily and with good accuracy. However, the relationship between $D_{M}$ and $D_{S}$ is not well established.

At equilibrium, thermodynamics interactions govern the composition of the interfacial region. In systems where the polymers are chemically different, the Gibbs free energy of mixing also contains a term corresponding to the enthalpy of mixing. The chemical potential gradient is considered as the driving force for diffusion. Its expression is given by the following equation [29]:

$$
\left(\frac{\partial \Delta \mu}{\partial \phi}\right)=\mathrm{kT} \cdot\left[\frac{1}{\mathrm{~N}_{\mathrm{A} .} \phi}+\frac{1}{\mathrm{~N}_{\mathrm{B} .}(1-\phi)}+2 \cdot|\chi|\right]
$$


where $\Delta \mu=\mu_{\mathrm{A}}-\mu_{\mathrm{B}}$ is the chemical potential difference

$\phi$ is the volume fraction of polymer A

$\mathrm{N}_{\mathrm{i}}$ is the number of repeat units of the polymer $\mathrm{i}$

$\chi$ is the Flory-Huggins interaction parameter between $\mathrm{A}$ and $\mathrm{B}$

Concentration and volume fraction are related by the expression $\phi=C \cdot a^{3}$, where $\mathrm{a}^{3}$ is the volume per monomer.

The first two terms in the square brackets in equation (2.1.a) represent the entropic contribution whereas the last one represents the enthalpic contribution to the molar free energy. The chemical potential gradient is directly proportional to the Flory-Huggins interaction parameter describing the molecular interactions of the two polymers. In the case of specific interactions, such as hydrogen bonding, $\chi$ is negative and as a consequence, the enthalpy of interaction is low, favorising the interdiffusion of the two species.

Two diffusion equations, which are not in agreement with each other, have been proposed in relating $D_{M}$ and $D_{S}$ for entangled chains. Their expressions are given below:

$$
\begin{aligned}
& \mathrm{D}_{\mathrm{MS}}=\frac{1}{\left(\frac{1-\phi}{\mathrm{D}_{\mathrm{SA}} \cdot \mathrm{N}_{\mathrm{A}}}+\frac{\phi}{\mathrm{D}_{\mathrm{SB}} \mathrm{N}_{\mathrm{B}}}\right)} \cdot \phi \cdot(1-\phi) \cdot \frac{1}{\mathrm{kT}} \cdot\left(\frac{\partial \mu}{\partial \phi}\right) \\
& \text { DMF }_{\mathrm{M}}=\left[\mathrm{D}_{\mathrm{SA}} \cdot \mathrm{N}_{\mathrm{A}} \cdot(1-\phi)+\mathrm{D}_{\mathrm{SB}} \cdot \mathrm{N}_{\mathrm{B}} \cdot \phi\right] \cdot \phi \cdot(1-\phi) \cdot \frac{1}{\mathrm{kT}} \cdot\left(\frac{\partial \mu}{\partial \phi}\right)
\end{aligned}
$$

where $D_{M S}$ is the interdiffusion coefficient for the slow theory $\mathrm{D}_{\mathrm{MF}}$ is the interdiffusion coefficient for the fast theory $\mathrm{D}_{\mathrm{SA}}$ is the self-diffusion coefficient of A chains $\mathrm{D}_{\mathrm{SB}}$ is the self-diffusion coefficient of $\mathrm{B}$ chains 
The equation (2.1.b) is known as the slow theory of diffusion since the diffusion is dominated by the slower diffusing component. This model has been derived by De Gennes [99], Brochard et al. [100], and Binder [101] by considering a dynamic version of the random phase approximation and by including vacancies into the lattice model. The slow theory assumes local incompressibility and also that the component fluxes are equal and opposite $\left(\mathrm{J}_{\mathrm{A}}=-\mathrm{J}_{\mathrm{B}}\right)$ during the diffusion process, i.e. that the interface remains symmetrical. This model is based on a mathematical approach and does not consider physical interactions. Figure 2.1 illustrates the mathematical concept behind the slow theory.

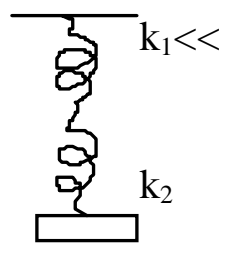

$$
\mathrm{k} \approx \mathrm{k}_{1}
$$

Figure 2.1: Schematic of the slow theory of diffusion

The equation (2.1.c) refers to the fast theory of diffusion in which the faster moving component controls the diffusion and swells the slower component. This model has been proposed by Crank [102], Kramer et al. [57], and later by Sillescu [103]. The fast diffusion theory also considers vacancies in a lattice model, but it assumes that local density inhomogeneities are rapidly relaxed, and thus the gradient of the chemical potential of the vacancies vanishes. There is an additional vacancy flux besides the fluxes of components A and B. Figure 2.2 shows the mathematical concept behind the fast theory.

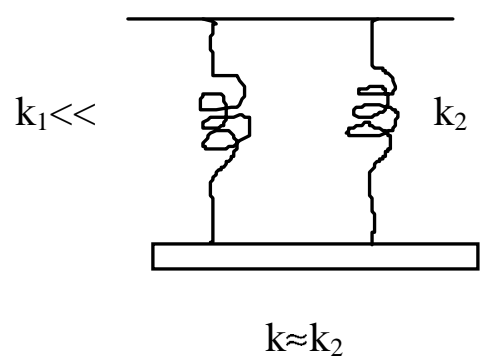

Figure 2.2: Schematic of the fast theory of diffusion 
Both theories are limiting cases. Indeed, they contain several limiting assumptions, such as no correlation factor for the self-diffusion process and the assumption that the monomeric friction coefficients are independent of composition.

\subsection{4 - COMPARISON BETWEEN THEORIES AND EXPERIMENTS}

Experimental results have been compared with the predictions of the fast and the slow theories of diffusion. There are results in the literature which agree with the slow theory of diffusion (see Table 2.2), and there are also results which agree with the fast theory of diffusion (see Table 2.3), and there are some that disagree with both theories (see Table 2.4).

Table 2.2: Results which agree with the slow theory of diffusion

\begin{tabular}{|l|l|l|}
\hline Materials & Technique & Reference \\
\hline PMMA and poly(styrene-co-acrylonitrile) & Ellipsometry & 98 \\
\hline PMMS / PS & Photon correlation spectroscopy & 92 \\
\hline Polytetramethyl carbonate / deuterated PS & Neutron scattering & 66 \\
\hline
\end{tabular}

Table 2.3: Results which agree with the fast theory of diffusion

\begin{tabular}{|l|l|l|}
\hline Materials & Technique & Reference \\
\hline PMMA and poly(styrene-co-acrylonitrile) & Forward recoil spectrometry & 55 \\
\hline PDMS / PEMS & Photon correlation spectroscopy & 83 \\
\hline PS / poly(xylenyl ether) & Forward recoil spectrometry & 56 \\
\hline $\begin{array}{l}2 \text { hydrogenated polybutadienes of different } \\
\text { molecular weights }\end{array}$ & Infrared microdensitometry & 36 \\
\hline
\end{tabular}


Table 2.4: Results which disagree with both the slow and the fast theories of diffusion

\begin{tabular}{|c|c|c|c|}
\hline Materials & Technique & Results & Reference \\
\hline $\begin{array}{l}\text { Deuterated } \\
\text { polystyrene and } \\
\text { PMMA }\end{array}$ & $\begin{array}{l}\text { Nuclear reaction } \\
\text { analysis }\end{array}$ & $\begin{array}{l}\text { Slow theory until } 10^{\circ} \mathrm{C} \text { above } \mathrm{T}_{\mathrm{g}} \text {, } \\
\text { fast theory at high temperature. } \\
\text { A function describing a thermal } \\
\text { transition between the two } \\
\text { theories was derived. }\end{array}$ & 96 \\
\hline \multirow[t]{2}{*}{$\begin{array}{l}\text { PS/PVME, PB/SBR } \\
\text { PI/SBR blends }\end{array}$} & Light scattering & $\begin{array}{l}\text { Slow mode at large molecular } \\
\text { weight, fast mode at low } \\
\text { molecular weight. } \\
\text { Good agreement with [105]. }\end{array}$ & 104 \\
\hline & $\begin{array}{l}\text { Dynamic scattering } \\
\text { experiments }\end{array}$ & $\begin{array}{l}\text { Slow theory as } T \rightarrow T_{g}, \\
\text { fast theory far above } T_{g} \text {. }\end{array}$ & 105 \\
\hline \multirow[t]{2}{*}{$\begin{array}{l}\text { PS / poly(vinyl } \\
\text { methyl ether) }\end{array}$} & $\begin{array}{l}\text { ATR-FTIR } \\
\text { spectroscopy }\end{array}$ & $\begin{array}{l}\text { A model was developed for } \\
\text { polymers with dissimilar physical } \\
\text { properties. }\end{array}$ & 73 \\
\hline & Theoretical & $\begin{array}{l}\text { Slow theory far above } T_{g} \text {. } \\
\text { A linear combination of the slow } \\
\text { and the fast modes was derived. }\end{array}$ & 106 \\
\hline
\end{tabular}

\subsection{5 - CONCLUSION}

The most frequently employed technique used to study the mutual interdiffusion at a (polymer/polymer) interface is ion-beam spectrometry, in other words Rutherford backscattering spectrometry (RBS), forward recoil spectrometry (FRES), and secondary ion mass spectrometry (SIMS). These three techniques allow the determination of the concentration profile as a function of depth, but are not always applicable to the vast 
majority of polymeric materials since the atomic compositions are not always distinguishable. Furthermore, the samples are damaged by the use of these techniques.

However, a non-destructive technique, Attenuated Total Reflectance (ATR) spectroscopy, has been successfully applied in the last decade to measure diffusion coefficients in polymers $[19,20,21,22,23,24,25,26]$. This technique will be used in this research to study the molecular interdiffusion at a poly(vinyl pyrrolidone)/vinyl ester (PVP/VE) interface.

\section{2 - FTIR-ATR SPECTROSCOPY}

The principles, practice and applications of spectroscopy, in general, are well documented in literature [107], as is polymer spectroscopy [108]. Spectroscopy is a

method studying the interaction of energy with a particular system, which produces a response that can be interpreted in terms of physical interactions in the system and chemical structure of the system.

This section provides a brief overview of infrared spectroscopy, Fourier transform infrared spectroscopy, and attenuated total reflectance spectroscopy.

\subsection{1 - INFRARED SPECTROSCOPY (IR)}

Three regions within the infrared region can be distinguished: the near-infrared region (from 13,300 $\mathrm{cm}^{-1}$ to $4,000 \mathrm{~cm}^{-1}$ ), the mid-infrared region (from 4,000 $\mathrm{cm}^{-1}$ to 400 $\mathrm{cm}^{-1}$ ) and the far-infrared region (from $400 \mathrm{~cm}^{-1}$ to $10 \mathrm{~cm}^{-1}$ ). Infrared spectroscopy measures the molecular vibrations of molecules. The molecules must have a permanent dipole moment in order to interact with the infrared radiation, to vibrate and to absorb energy. When a sample absorbs infrared radiation at a given wavenumber, there is a corresponding decrease in the radiation intensity, which can be mathematically transformed into an absorption band. The infrared spectrogram presents, indeed, the absorption characteristics of a molecule, also called bands or peaks. There is a correlation between the functional groups of a molecule and the wavenumbers at which they absorb 
infrared radiation. The carbonyl group stretch, for instance, always occurs between 1600 $\mathrm{cm}^{-1}$ and $1700 \mathrm{~cm}^{-1}$, depending upon the local environment of the carbonyl moiety. Infrared spectroscopy is, therefore, used to characterize molecular structure, and to detect and to identify the presence of intermolecular interactions which cause frequency shifts in some cases.

\subsection{2 - FTIR SPECTROSCOPY}

Fourier Transform Infrared (FTIR) spectroscopy is said to be the most versatile, fast, inexpensive, and conclusive method for surface and bulk characterization. As a result, FTIR spectrometers are found in most laboratories. The main component of the FTIR spectrometer is the Michelson interferometer [109, 110], invented in 1880 by Albert Abraham Michelson, who was awarded the Nobel Prize in Physics in 1907. The major advantage of a FTIR spectrometer over a dispersive infrared spectrometer is that the signal-to-noise ratio (SNR) is quite high. SNR is defined as the ratio of the amplitude of a spectral band at any point to the amplitude of the noise at some baseline point nearby in the spectrum. Noise is observed when there are some fluctuations in the spectrum above and below the baseline.

FTIR spectroscopy has been classified into two major areas: non-reflection techniques and reflection techniques. Table 2.5 distinguishes different techniques within both areas while Table 2.6 gives some information about their penetration depth range and surface requirements.

Table 2.5: Non-reflection and reflection techniques

Non-reflection techniques:

Emission spectroscopy (EMS)

Photothermal beam deflection spectroscopy (PBDS)

Photoacoustic spectroscopy (PAS)

Transmission spectroscopy 
Reflection techniques:

External spectroscopy (ERS)

Reflection-absorption spectroscopy (RAS)

Grazing incidence reflection (GIR)

Internal spectroscopy (IRS)

Multiple internal reflection (MIR)

Frustrated total reflection (FTR)

Attenuated total reflectance (ATR)

Diffuse reflectance (DRIFT)

Table 2.6: Penetration depth and surface requirements for some FTIR surface techniques (from [111])

\begin{tabular}{|c|c|c|}
\hline Technique & Penetration depth range & Surface \\
\hline RAS & submonolayer to $2 \mu \mathrm{m}$ & coated metallic \\
\hline PAS, PBDS & $200 \AA$ to $50 \mu \mathrm{m}$ & any shape \\
\hline EMS & $100 \AA$ to $5 \mu \mathrm{m}$ & any surface \\
\hline DRIFT & monolayer to few $\mu \mathrm{m}$ & powder, fiber \\
\hline ATR & $40 \AA$ to $3 \mu \mathrm{m}$ & smooth film \\
\hline
\end{tabular}

\subsection{3 - FTIR-ATR SPECTROSCOPY}

Attenuated Total Reflectance (ATR) spectroscopy is an internal reflection technique used in conjunction with an FTIR. The name of the technique was first given by Fahrenfort, but it has been redefined by The American Society for Testing and Materials (ASTM). Its definition is given in Appendix A. 
ATR spectroscopy is often considered as a technique to study the surface of different materials, such as thin films or opaque solids. Its principles are given in the following section.

\section{3 - OPTICAL PRINCIPLES OF ATR SPECTROSCOPY}

Harrick [112] and others $[110,113]$ have presented the fundamental principles of Attenuated Total Reflectance (ATR) spectroscopy in detail.

The ATR technique requires an internal reflection element (IRE), also called the denser or propagating medium, and often referred to as the ATR crystal. The sample, called the rarer medium, because of its lower refractive index, is in contact with this crystal. Thus an absorbing medium is in contact with a reflecting one.

As shown in Figure 2.3, an incident beam enters the ATR crystal from one of the side faces and is totally reflected at the interface with the sample. The beam then travels inside the ATR crystal and finally exits from the other side face.

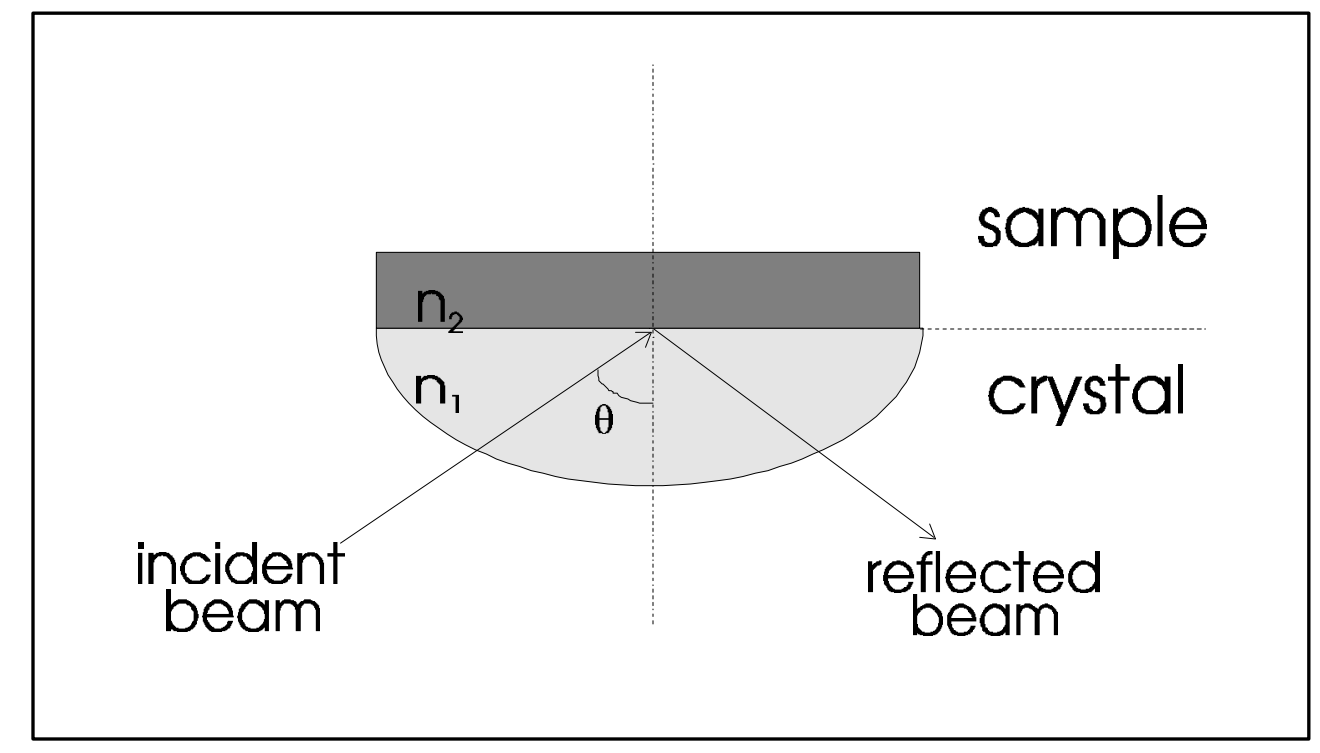

Figure 2.3: Schematic representation of total internal reflection 
The nature of the interaction at the interface depends on the refractive indices of the ATR crystal and the sample, $\mathrm{n}_{1}$ and $\mathrm{n}_{2}$ respectively.

For an absorbing material, the refractive index is a complex quantity $\tilde{\mathrm{n}}$, which is defined below:

$$
\tilde{\mathrm{n}}=\mathrm{n}+\mathrm{i} \cdot \mathrm{k}
$$

where $\mathrm{n}$ is the real component of the refractive index

(a constant at a particular frequency)

$\mathrm{k}$ is the extinction coefficient, also called the absorption index

For organic materials in the mid-infrared region, the refractive index is usually about 1.5 .

The angle of incidence, $\theta$, is defined as the angle with respect to the normal to the face of the IRE crystal.

Total internal reflection occurs only if $\theta \geq \theta_{c}$, where the critical angle $\theta_{c}$ is given by the equation:

$$
\begin{aligned}
& \qquad \vartheta_{\mathrm{c}}=\sin ^{-1}\left(\frac{\mathrm{n}_{2}}{\mathrm{n}_{1}}\right) \\
& \text { where } \mathrm{n}_{1}>\mathrm{n}_{2} \text { so that }\left(\mathrm{n}_{2} / \mathrm{n}_{1}\right)<1 \\
& \mathrm{n}_{1} \text { is the refractive index of the ATR crystal } \\
& \mathrm{n}_{2} \text { is the refractive index of the sample }
\end{aligned}
$$

In order to avoid some distortions in the band shapes, the angle of incidence has to be maintained well above the critical angle.

When the infrared radiation is totally reflected at the interface between the crystal and the sample, an evanescent wave, which is the wave of infrared radiation inside the ATR crystal, penetrates a small distance in the sample. It represents the superposition of 
the incident and reflected beams at the interface between the IRE and the sample. The total distance sampled by the evanescent wave is actually much longer than the so called penetration depth, which is defined as the distance at which the relative electric field is reduced by a factor $\mathrm{e}^{-1}$.

Assuming a small extinction coefficient (the sample is a non-absorbing medium and $\mathrm{k}=0$ in the equation (2.3.a)), the penetration depth $\mathrm{dp}$ in $\mu \mathrm{m}$ of the IR radiation is given by:

$$
\mathrm{d}_{\mathrm{p}}=\frac{\lambda}{2 \pi \cdot \mathrm{n}_{1 \cdot} \sqrt{(\sin \vartheta)^{2}-\left(\frac{\mathrm{n}_{2}}{\mathrm{n}_{1}}\right)^{2}}}
$$

where $\lambda$ is the IR wavelength of the light in free space in $\mu \mathrm{m}$

$\lambda=(10,000 / \sigma)$ where $\sigma$ is the wavenumber in $\mathrm{cm}^{-1}$

$\theta$ is the angle of incidence

$\mathrm{n}_{1}$ is the refractive index of the ATR crystal

$\mathrm{n}_{2}$ is the refractive index of the sample in contact with the ATR crystal

At $\theta \approx \theta_{c}$, the approximations made for deriving the penetration depth equation (2.3.c) are no longer valid since the denominator is equal to zero.

A knowledge of the penetration depth is very important when working with solids since the chemical composition of solids can vary with depth. By changing the angle of incidence it is possible to study surfaces at various depths. The penetration depths can be calculated with the use of equation (2.3.c). From this equation, we see that $d_{p}$ decreases as $\theta$ increases at constant $n_{1}, n_{2}$, and $\lambda$. This is illustrated in Figure 2.4 for $n_{1}=2.42$ and $n_{2}=$ 1.53. On the other hand, $\mathrm{d}_{\mathrm{p}}$ increases proportionally with $\lambda$ at constant $\theta, \mathrm{n}_{1}$ and $\mathrm{n}_{2}$, as demonstrated in Figure 2.5 for $\mathrm{n}_{1}=2.42$ and $\mathrm{n}_{2}=1.53$. 


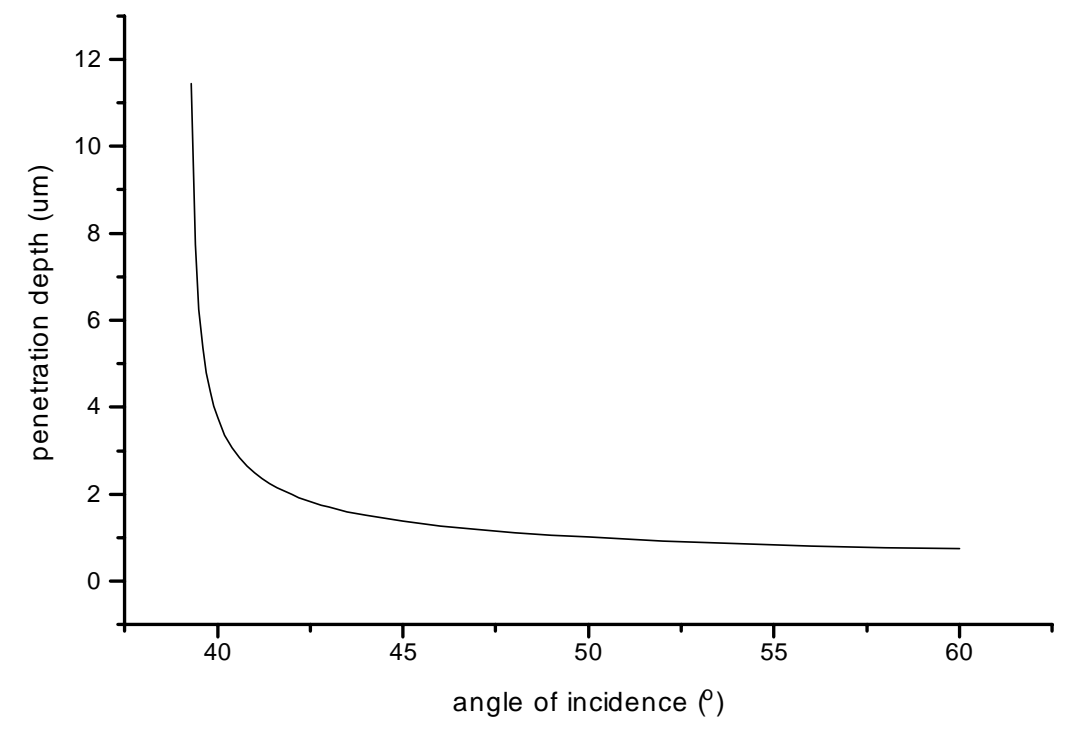

Figure 2.4: Penetration depth, $d_{p}$, versus angle of incidence $\theta$ at $1507 \mathrm{~cm}^{-1}$

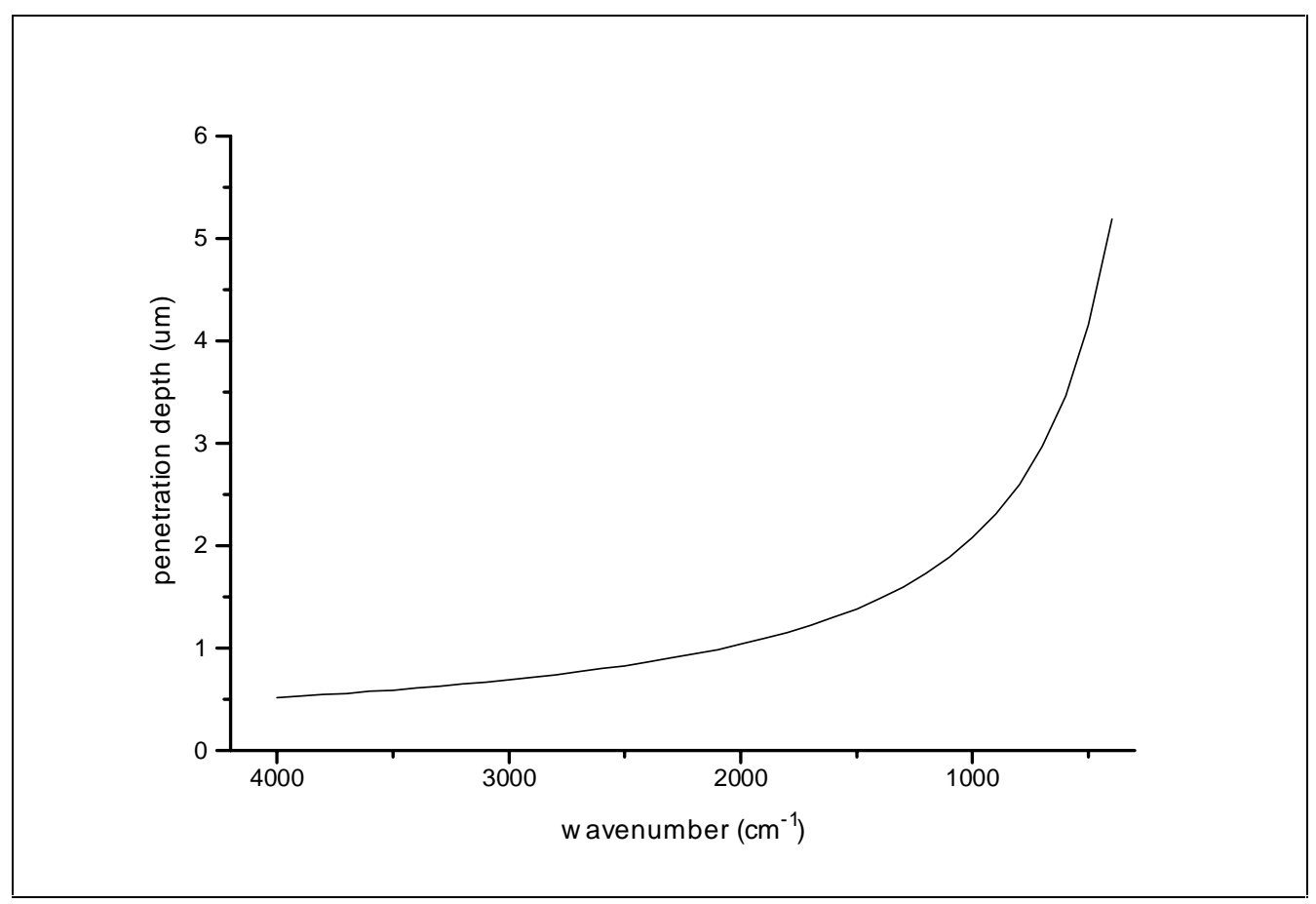

Figure 2.5: Penetration depth, $d_{p}$, versus wavenumber for $\theta=45^{\circ}$ 
As shown in Figure 2.6, the electric field magnitude E undergoes an exponential decay (the largest contribution to the absorption intensity comes, thus, from the material which is close to the surface), and the rate of decay is defined as:

$$
E=E_{o} \cdot e^{-(z / d p)}
$$

where $\mathrm{E}_{\mathrm{o}}$ is the electric field magnitude value at the (sample/ATR crystal) $\mathrm{z}$ is the distance from the (sample/ATR crystal) interface $\mathrm{dp}$ is the penetration depth

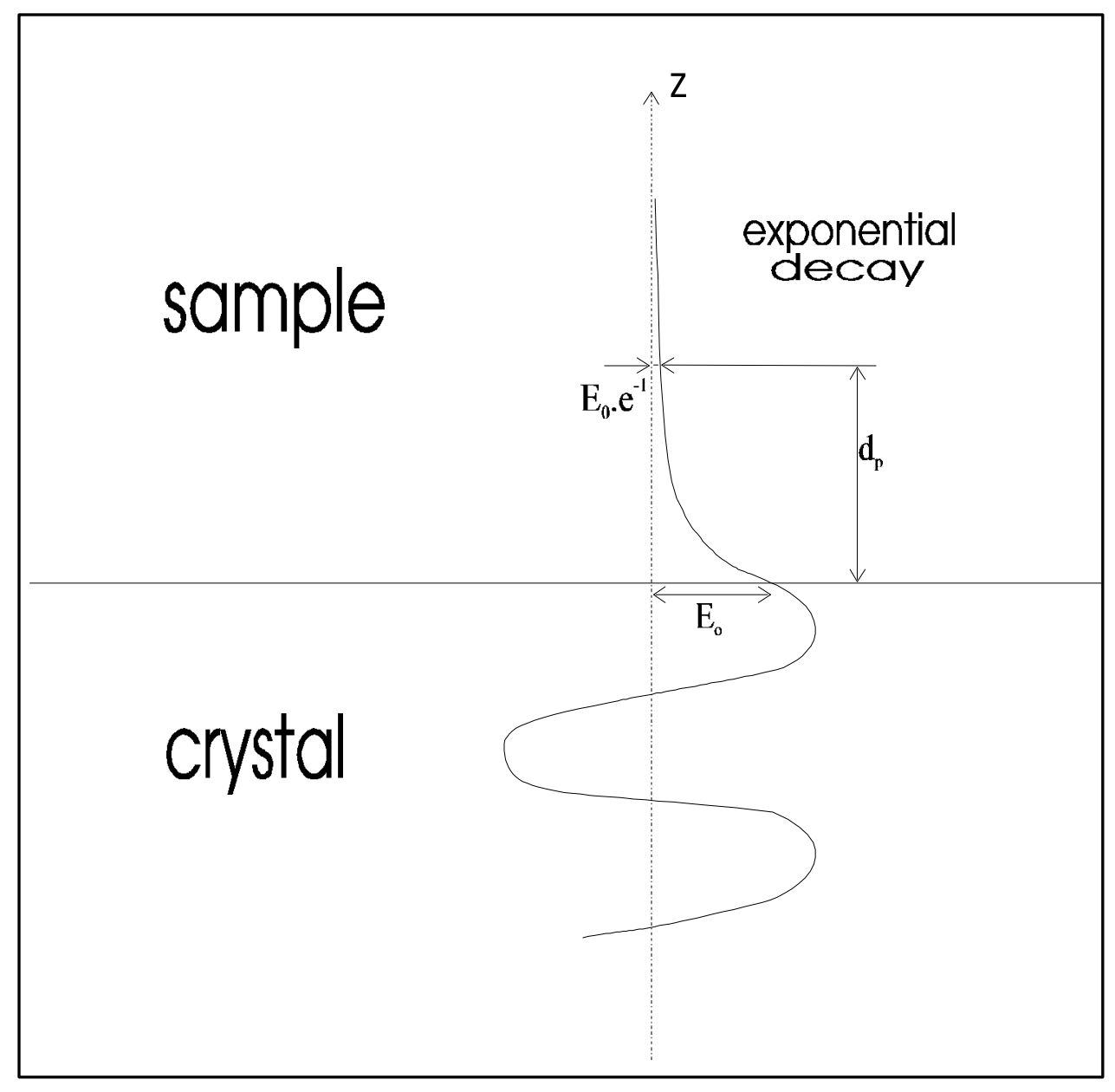

Figure 2.6: Schematic of the evanescent wave near the interface 
The square of the electric field magnitude gives us its intensity, I, which can be related to the absorbance of light [114]. This will be discussed in Subsection 2.4.1.

To summarize, the sample is able to absorb light of that of the evanescent wave at particular wavenumbers. Some energy is, thus, removed from the reflected beam and hence, the name Attenuated Total Reflectance (ATR). The decrease in the reflected beam intensity results in an absorption spectrum. The spectrum can then be interpreted in terms of the physical and chemical structure and properties of the system.

\section{4 - MEASURING DIFFUSION IN POLYMERS BY ATR SPECTROSCOPY}

The FTIR-ATR technique has been used to measure diffusion coefficients in polymers $[19,20,21,22,23,24,25,26]$ only in recent years. Its historical development can be found in the literature [19]. The first attempts $[115,116]$ were made in the early 1980 's, but they were not conclusive because valid equations were not incorporated into the model for analysis. In 1984, an article was published by Brandth et al. [117] about an ATR experiment done in situ concerning the diffusion of small molecules in polyethylene. The analysis model was then given and value of the diffusion coefficient was obtained. However, since this article was published in German and not in English, it did not attract attention. In 1988, Xu and Balik [118] described a numerical method involving measurement of the absorbance bands as a function of time, without even referencing to the work previously done by Brandth et al.. However, their experimental data could not be accurately converted to diffusion coefficient because the experiments were not done in situ. As a result, some researchers studied the diffusion behavior in other polymeric

materials. But no other articles reporting the use of the ATR technique for measuring diffusion coefficient were published until 1992.

This section starts by presenting the mathematical details inherent in the determination of the expression of the absorbance in the ATR measurement. The following subsection discusses the different transport models. One of them, the Fickian 
diffusion model, is generally valid for most of the systems and is therefore expected to model diffusion in polymers. The diffusion model is formulated in the third subsection, assuming a Fickian behavior. A review of the literature is provided in the fourth subsection for both the diffusion of small molecules into polymers and the diffusion of polymers within polymers. The final subsection summarizes the advantages and limitations of ATR spectroscopy.

\subsection{1 - EXPRESSION OF THE ABSORBANCE}

The absorbance is a measure of the quantity of energy absorbed by a sample. The Beer-Lambert law [107, 109, 110] gives a relationship between the absorbance of a band and the light intensity valid for transmission spectroscopy:

$$
\mathrm{A}=-\log \mathrm{T}=\log \left(\frac{\mathrm{I}_{0}}{\mathrm{I}}\right)=\varepsilon . \mathrm{C} .1
$$

where $\mathrm{A}$ is the absorbance

$\mathrm{T}$ is the transmittance

$I_{o}$ is the intensity measured with no sample in the beam

(from the background spectrum)

I is the intensity measured with a sample in the beam

(from the sample single beam spectrum)

$\varepsilon$ is the absorptivity

$\mathrm{C}$ is the concentration of the absorbing material

(c.C) is the absorption coefficient. It is equal to $((4 . \pi . n . \mathrm{k}) / \lambda)$

1 is the thickness of the absorption path 
From the equation (2.4.a) we get:

$$
\left(\frac{\mathrm{I}}{\mathrm{I}_{\mathrm{o}}}\right)=\exp (-\mathrm{A} \cdot \ln 10)
$$

If we assume that the maximum absorbance value of the peak is less than $(\ln 10)^{-1}$, in other words that only weak absorption occurs, then:

$\exp (-\mathrm{A} \cdot \ln 10)=1-\mathrm{A} \cdot \ln 10+\frac{(\mathrm{A} \cdot \ln 10)^{2}}{2 !}-\frac{(\mathrm{A} \cdot \ln 10)^{3}}{3 !}+\ldots \ldots \ldots \ldots \ldots \ldots(1-\mathrm{A} \cdot \ln 10)$

Substituting (2.4.c) into (2.4.b) and taking a derivative, we get:

$$
d I=-(\ln 10) \cdot(I 0) \cdot d A
$$

The Beer-Lambert law (2.4.a) is equivalent to:

$$
\log I 0-\log I=\varepsilon . C . l
$$

or $\quad-\int_{\mathrm{Io}}^{\mathrm{I}}\left(\frac{1}{\ln 10}\right) \cdot\left(\frac{1}{\mathrm{I}}\right) \cdot \mathrm{dI}=\int_{0}^{1} \varepsilon \cdot \mathrm{C} \cdot \mathrm{dz} \quad$ (assuming constant concentration)

Differentiating the equation (2.4.f) gives:

$$
\begin{gathered}
-\left(\frac{1}{\ln 10}\right) \cdot\left(\frac{1}{I}\right) \cdot d I=\varepsilon \cdot C \cdot d z \\
\quad \text { or } d I=-\varepsilon \cdot C \cdot I \cdot(\ln 10) \cdot d z
\end{gathered}
$$


Combining (2.4.d) and (2.4.h) we have:

$$
\begin{gathered}
-\varepsilon . C . I .(\ln 10) \cdot d z=-(\ln 10) \cdot(\text { Io }) \cdot d A \\
\quad \text { or } d A=\text { E.C. }\left(\frac{I}{I_{0}}\right) \cdot d z
\end{gathered}
$$

Using the ATR relations discussed in Section 2.3, $E^{2}=I$ and $E=E_{o} \cdot \exp \left(\frac{-z}{d_{p}}\right)$, we get:

$$
\left(\frac{I}{I o}\right)=\left(\frac{E o^{2}}{I o}\right) \exp \left(\frac{-2 z}{d p}\right)
$$

Substituting (2.4.j) into (2.4.i), we obtain:

$$
d A=\varepsilon . C \cdot\left(\frac{E o^{2}}{I o}\right) \cdot \exp \left(\frac{-2 z}{d p}\right) \cdot d z
$$

If several reflections occur, then: $\left(\frac{I}{I o}\right)=R^{N}$

$$
\begin{aligned}
& \text { where } \mathrm{R} \text { is the reflectivity } \\
& \mathrm{N} \text { is the number of reflections }
\end{aligned}
$$

If the concentration of the absorbing species is not distributed uniformly, then the absorbance per unit area is:

$$
A=\int_{0}^{\infty} N \cdot \varepsilon \cdot\left(\frac{E^{2}}{I o}\right) \cdot \exp \left(\frac{-2 z}{d p}\right) \cdot C(z) \cdot d z
$$


Thus, the expression of the absorbance for the ATR configuration as a function of time is given by $[19,119,120,121,122]$ :

$$
A(t)=\int_{0}^{\infty} \alpha \cdot \exp \left(\frac{-2 z}{d_{p}}\right) \cdot C(z, t) \cdot S \cdot d z
$$

where $A(t)$ is the absorbance at any time

$\mathrm{t}$ is the time

$\alpha$ is the characteristic absorptivity or oscillator strength

a constant which includes the molar extinction coefficient and the number of reflections

$\mathrm{S}$ is the cross-sectional area over which the measurement occurs $\mathrm{dp}$ is the penetration depth $\mathrm{Z}$ is the distance from the ATR crystal $\mathrm{C}(\mathrm{z}, \mathrm{t})$ is the concentration profile at a particular time $\mathrm{t}$

The exponential term in (2.4.1) represents the exponential decay of the evanescent wave. Complete expressions of the absorbance valid for polarized radiation can be found in the literature [123].

\subsection{2 - DIFFUSION MODELS}

The dissolution of a polymer into another polymer can be tracked by measuring the intensity of characteristic absorbance bands of the components as a function of time for a given temperature. By looking at the equation (2.4.1), we see that the expression of the concentration profile, in which a difusion coefficient is involved, has to be determined in order to get a relationship between the absorbance and the diffusion coefficient .

It is necessary to apply the appropriate diffusion model to the transport behavior. Two models have been considered: the case I or Fickian diffusion, and the case II diffusion $[19,120]$. A combination of both of these models was also derived and can be 
found elsewhere [24]. The case I diffusion occurs when the rate of diffusion is much lower than the relaxation rate of the polymer, whereas the case II diffusion occurs when the diffusion is very rapid compared to the polymer relaxation time. The case II diffusion is independent of the concentration profile since it depends on the relaxation of the polymer material. The interface moves linearly with time for this case. The Fickian and the case II models represent highly idealized cases of diffusion, but one has to be aware that other models of diffusion also exist. Case III diffusion for instance occurs when the diffusion and relaxation rates are comparable.

\subsection{3 - THE FICKIAN MODEL}

The Fickian model is usually a good approximation. Indeed, most (polymer/polymer) and (polymer/solute) systems obey this behavior. Moreover, it is the easiest model to use. Example calculations can be found in the literature for this model [102].

This subsection starts by describing the interdiffusion system. The expressions of the Fickian behavior is then derived. Once the initial and boundary conditions have been established, the expression of the concentration profile is calculated. Finally, the mathematical details inherent in the determination of the diffusion model are presented.

\subsubsection{1 - The Interdiffusion System}

The experimental interdiffusion system is prepared by casting a thin polymeric film onto an ATR crystal and sandwiching it with a monomer sample. Figure 2.7 illustrates the schematic interdiffusion system, where $\mathrm{z}$ represents the distance from the crystal, $\mathrm{b}$ is the thickness of the polymer, and (a-b) the thickness of the monomer.

The interdiffusion direction is expected to be along the z-axis only. The polymer is so thin, covering the entire ATR crystal area, that a negligible amount of the monomer enters through the edges. 


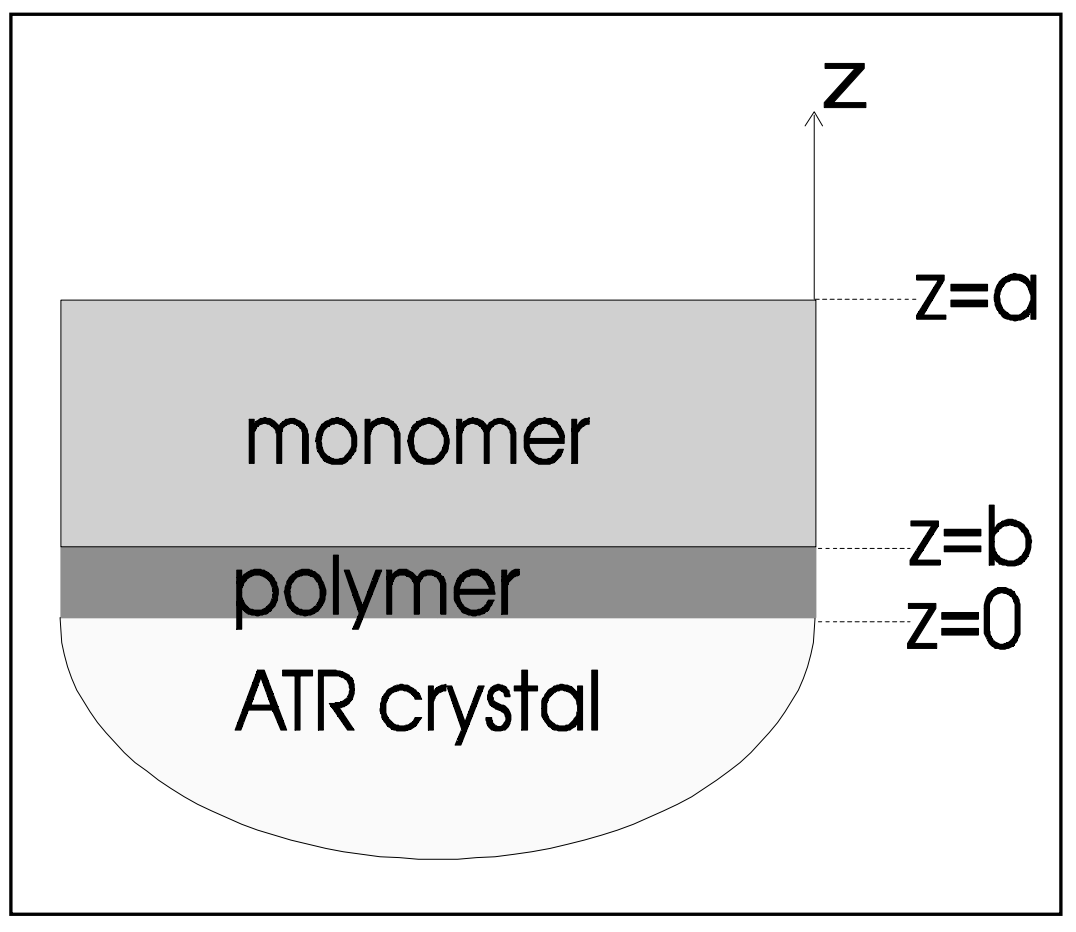

Figure 2.7: Schematic of the interdiffusion system

\subsubsection{2 - Fickian Diffusion}

The combination of Fick's second law for unsteady state and the continuity equation for one-dimensional molecular diffusion reduces to:

$$
\left(\frac{\partial \mathrm{C}}{\partial \mathrm{t}}\right)=\mathrm{D} \cdot\left(\frac{\partial^{2} \mathrm{C}}{\partial \mathrm{z}^{2}}\right)
$$

assumming that the diffusion coefficient $\mathrm{D}$ is constant. The equation has been simplified for the case where $\mathrm{D}$ depends only on temperature, and is independent of other parameters such as concentration, position, and thermal history.

The parameter $\mathrm{C}(\mathrm{z}, \mathrm{t})$ describes the change in the concentration profile of the polymer with time along the z-axis. 


\subsubsection{3 - Initial and Boundary Conditions}

In order to solve the equation (2.4.m), the appropriate initial and boundary conditions have to be established. Since the two layers are initially unmixed and since there is no flux across the boundaries (impermeable surfaces), initial and boundary conditions are defined as follows for the polymer:

$$
\begin{aligned}
& \mathrm{C}=0 \text { at } \mathrm{t}=0, \mathrm{~b}<\mathrm{z} \leq \mathrm{a} \\
& \mathrm{C}=\mathrm{C}_{\mathrm{o}} \text { at } \mathrm{t}=0,0 \leq \mathrm{z} \leq \mathrm{b} \\
& \frac{\partial \mathrm{C}}{\partial \mathrm{z}}(0, \mathrm{t})=\frac{\partial \mathrm{C}}{\partial \mathrm{z}}(\mathrm{a}, \mathrm{t})=0
\end{aligned}
$$

Assuming that the reduction of $\mathrm{C}(\mathrm{z}, \mathrm{t})$ is due to the intrusion of the monomer, the concentration of the monomer $\mathrm{C}_{\mathrm{m}}(\mathrm{z}, \mathrm{t})$ can be evaluated with the relation:

$$
\frac{C_{m}(z, t)}{C_{m o}}=1-\frac{C(z, t)}{C_{o}}
$$

where $\mathrm{C}_{\text {mo }}$ is the initial concentration of the monomer for $b<z \leq a$

\subsubsection{4 - Concentration Profile}

The solutions of the Fickian diffusion equation (2.4.m) can be obtained either by a Laplace transform or by the method of separation of the variables. It has been shown that the concentration profile of the polymer, within the penetration depth, given the initial and boundary conditions previously defined, can be expressed [102, 124] by the following equation:

$$
\frac{\mathrm{C}(\mathrm{z}, \mathrm{t})}{\mathrm{C}_{0}}=\frac{\mathrm{b}}{\mathrm{a}}+\frac{2}{\pi} \sum_{\mathrm{n}=1}^{\infty}\left\{\left(\frac{1}{\mathrm{n}}\right) \cdot \sin \left(\frac{\mathrm{n} \pi \mathrm{b}}{\mathrm{a}}\right) \cdot \cos \left(\frac{\mathrm{n} \pi \mathrm{z}}{\mathrm{a}}\right) \cdot \exp \left(\frac{-\mathrm{n}^{2} \pi^{2} \mathrm{Dt}}{\mathrm{a}^{2}}\right)\right\}
$$


where $\mathrm{C}(\mathrm{z}, \mathrm{t})$ is the concentration profile of the polymer

$\mathrm{C}_{0}$ is the initial concentration of the polymer

$\mathrm{Z}$ is the distance from the crystal

$\mathrm{t}$ is the time

$\mathrm{n}$ is the index of summation

$\mathrm{D}$ is the diffusion coefficient

a is the total thickness of the polymer and the monomer

$\mathrm{b}$ is the thickness of the polymer

Another equivalent expression of the concentration profile, convenient for small times, has been given [102] by the relation:

$$
C(z, t)=\frac{C_{0}}{2} \sum_{n=-\infty}^{\infty}\left\{\operatorname{erf} \frac{b+2 n a-z}{2 \sqrt{D t}}+\operatorname{erf} \frac{b-2 n a+z}{2 \sqrt{D t}}\right\}
$$

In order to get the equation (2.4.o), several assumptions were made. The two phases were assumed to be completely miscible. The interface was expected to remain parallel to the boundaries. Finally, the total thickness of the system was considered to stay constant during the course of the experiments; that is no volume changes were associated with the diffusion of the monomer into the polymer and thus, there was no volume change upon mixing. The equation (2.4.o) does not consider any difference in size between the two components of the system. 


\subsubsection{5 - The Diffusion Model}

FTIR spectrometers measure the absorbance and not the concentration. Nevertheless, absorbance and concentration can be related via the equation (2.4.1).

We have

$$
A(t)=\int_{0}^{a} \alpha \cdot S \cdot \exp \left(\frac{-2 z}{d p}\right) \cdot C(z, t) \cdot d z
$$

where $C(z, t)=\left(C_{0}\right) \cdot\left\{\frac{b}{a}+\frac{2}{\pi} \sum_{n=1}^{\infty}\left\{\left(\frac{1}{n}\right) \cdot \sin \left(\frac{n \pi b}{a}\right) \cdot \cos \left(\frac{n \pi z}{a}\right) \cdot \exp \left(\frac{-n^{2} \pi^{2} D t}{a^{2}}\right)\right\}\right\}$

By substituting the expression of the concentration profile of the polymer (2.4.0) in the integral of (2.4.1), the value of the absorbance at any time can be obtained.

Here, a, b, dp, D, $\alpha$ and S are assumed to be constant.

The initial absorbance and the absorbance at infinite time for the polymer were calculated.

$$
\begin{aligned}
A o= & \int_{0}^{b} \alpha \cdot C(z, 0) \cdot S \cdot \exp \left(\frac{-2 z}{d p}\right) \cdot d z+\int_{b}^{a} \alpha \cdot C \cdot(z, 0) \cdot S \cdot \exp \left(\frac{-2 z}{d p}\right) \cdot d z \\
= & \alpha \cdot C o \cdot S \cdot \int_{0}^{\mathrm{b}} \exp \left(\frac{-2 \mathrm{z}}{\mathrm{dp}}\right) \cdot \mathrm{dz} \\
= & \alpha \cdot \operatorname{Co\cdot S} \cdot\left(\frac{\mathrm{dp}}{2}\right) \cdot\left(1-\exp \left(\frac{-2 \mathrm{~b}}{\mathrm{dp}}\right)\right) \\
A_{\infty}= & \int_{0}^{a} \alpha \cdot S \cdot \exp \left(\frac{-2 z}{d p}\right) \cdot C(z, \infty) \cdot d z \\
& \quad \text { but } C(z, \infty)=\left(C_{0}\right) \cdot\left(\frac{b}{a}\right) \text { since } \exp \left(\frac{-n^{2} \pi^{2} D t}{a^{2}}\right)=0 \text { in equation (2.4.o) }
\end{aligned}
$$


Hence $A_{\infty}=\alpha \cdot C_{0} \cdot S \cdot\left(\frac{b}{a}\right) \cdot \int_{0}^{a} \exp \left(\frac{-2 z}{d p}\right) \cdot d z$

$$
=\alpha \cdot C_{0} \cdot S \cdot\left(\frac{b}{a}\right) \cdot\left(\frac{d p}{2}\right) \cdot\left(1-\exp \left(\frac{-2 a}{d p}\right)\right)
$$

Thus we get: $\quad\left(\frac{A_{\infty}}{A_{o}}\right)=\left(\frac{b}{a}\right) \cdot \frac{\left[1-\exp \left(\frac{-2 a}{d p}\right)\right]}{\left[1-\exp \left(\frac{-2 b}{d p}\right)\right]}$

We notice that $A_{t}=A_{\infty}+\alpha \cdot C_{0} \cdot S \cdot\left(\frac{2}{\pi}\right) \cdot K_{1}$

$$
\text { where } \mathrm{K}_{1}=\int_{0}^{\mathrm{a}} \exp \left(\frac{-2 \mathrm{z}}{\mathrm{dp}}\right) \cdot \sum_{\mathrm{n}=1}^{\infty}\left\{\left(\frac{1}{\mathrm{n}}\right) \cdot \sin \left(\frac{\mathrm{n} \pi \mathrm{b}}{\mathrm{a}}\right) \cdot \cos \left(\frac{\mathrm{n} \pi \mathrm{z}}{\mathrm{a}}\right) \cdot \exp \left(\frac{-\mathrm{n}^{2} \pi^{2} \mathrm{Dt}}{\mathrm{a}^{2}}\right)\right\} \cdot d \mathrm{z}
$$

We calculate the ratio $\frac{A(t)-A_{o}}{A_{\infty}-A_{o}}$ :

$$
\frac{A(t)-A_{0}}{A_{\infty}-A_{0}}=1+\left(\frac{\alpha \cdot C_{o} \cdot S \cdot\left(\frac{2}{\pi}\right) \cdot K_{1}}{A_{\infty}-A_{\circ}}\right)
$$

But $\quad \mathrm{A}_{\infty}-\mathrm{A}_{\circ}=-\alpha . \mathrm{C}_{\mathrm{o} . S} \mathrm{~S} \cdot\left(\frac{\mathrm{dp}}{2}\right) \cdot\left[\left(1-\exp \left(\frac{-2 \mathrm{~b}}{\mathrm{dp}}\right)\right)-\left(\frac{\mathrm{b}}{\mathrm{a}}\right) \cdot\left(1-\exp \left(\frac{-2 \mathrm{a}}{\mathrm{dp}}\right)\right)\right]$

$$
\text { Then } \frac{A(t)-A_{0}}{A_{\infty}-A_{0}}=1+\left(\frac{\left(\frac{2}{\pi}\right) \cdot K_{1}}{\left(\frac{-d p}{2}\right) \cdot \text { deno min ator }}\right)
$$


where denominator $=\left[\left(1-\exp \left(\frac{-2 b}{d p}\right)\right)-\left(\frac{b}{a}\right) \cdot\left(1-\exp \left(\frac{-2 a}{d p}\right)\right)\right]$

We integrate $K_{1}$ by parts and after some mathematical manipulations we find that:

$$
\begin{aligned}
& \mathrm{K}_{1}=\left(\frac{\mathrm{dp}}{2}\right) \cdot \mathrm{K}_{2}-\left(\frac{\mathrm{dp}}{2}\right) \cdot\left(\frac{\pi}{\mathrm{a}}\right) \cdot \mathrm{K}_{3} \\
& \text { where } \quad K_{2}=\sum_{n=1}^{\infty}\left\{\left(\frac{1}{n}\right) \cdot \sin \left(\frac{n \pi b}{a}\right) \cdot \exp \left(\frac{-n^{2} \pi^{2} D t}{a^{2}}\right) \cdot\left[1-(-1)^{n} \cdot \exp \left(\frac{-2 a}{d p}\right)\right]\right\} \\
& \text { and } \quad \mathrm{K}_{3}=\int_{0}^{\mathrm{a}} \exp \left(\frac{-2 \mathrm{z}}{\mathrm{dp}}\right) \cdot \sum_{\mathrm{n}=1}^{\infty}\left\{\sin \left(\frac{\mathrm{n} \pi \mathrm{b}}{\mathrm{a}}\right) \cdot \sin \left(\frac{\mathrm{n} \pi \mathrm{z}}{\mathrm{a}}\right) \cdot \exp \left(\frac{-\mathrm{n}^{2} \pi^{2} \mathrm{Dt}}{\mathrm{a}^{2}}\right)\right\} \cdot d \mathrm{dz} \\
& =1-\left(\frac{\left(\frac{2}{\pi}\right) \cdot\left(\mathrm{K}_{2}-\left(\frac{\pi}{\mathrm{a}}\right) \cdot \mathrm{K}_{3}\right)}{\text { deno min ator }}\right)
\end{aligned}
$$

Continuing integrating by parts we get:

$$
\mathrm{K}_{3}=\left(\frac{\mathrm{dp}}{2}\right) \cdot\left(\frac{\pi}{\mathrm{a}}\right) \cdot \mathrm{K}_{4}
$$




$$
\text { where } \mathrm{K}_{4}=\int_{0}^{\mathrm{a}} \exp \left(\frac{-2 \mathrm{z}}{\mathrm{dp}}\right) \cdot \sum_{\mathrm{n}=1}^{\infty}\left\{\mathrm{n} \cdot \sin \left(\frac{\mathrm{n} \pi \mathrm{b}}{\mathrm{a}}\right) \cdot \cos \left(\frac{\mathrm{n} \pi \mathrm{z}}{\mathrm{a}}\right) \cdot \exp \left(\frac{-\mathrm{n}^{2} \pi^{2} \mathrm{Dt}}{\mathrm{a}^{2}}\right)\right\} \cdot d \mathrm{z}
$$

Then

$$
\frac{\mathrm{A}(\mathrm{t})-\mathrm{A}_{0}}{\mathrm{~A}_{\infty}-\mathrm{A}_{0}}=1-\left(\frac{\left(\frac{2}{\pi}\right) \cdot\left(\mathrm{K}_{2}-\left(\frac{\pi}{\mathrm{a}}\right)^{2} \cdot\left(\frac{\mathrm{dp}}{2}\right) \cdot \mathrm{K}_{4}\right)}{\text { deno min ator }}\right)
$$

$$
=1-\left(\frac{\left(\frac{2}{\pi}\right) \cdot \mathrm{K}_{2}-\left(\frac{\pi \cdot \mathrm{dp}}{\mathrm{a}^{2}}\right) \cdot \mathrm{K}_{4}}{\text { deno min ator }}\right)
$$

Integrating $\mathrm{K}_{4}$ by parts we obtain:

$$
\begin{aligned}
& \mathrm{K}_{4}=\left(\frac{\mathrm{dp}}{2}\right) \cdot \mathrm{K}_{5}-\left(\frac{\mathrm{dp}}{2}\right) \cdot\left(\frac{\pi}{\mathrm{a}}\right) \cdot \mathrm{K}_{6} \\
& \text { where } K_{5}=\sum_{n=1}^{\infty}\left\{n \cdot \sin \left(\frac{n \pi b}{a}\right) \cdot \exp \left(\frac{-n^{2} \pi^{2} D t}{a^{2}}\right) \cdot\left[1+(-1)^{n+1} \cdot \exp \left(\frac{-2 a}{d p}\right)\right]\right\} \\
& \text { and } \quad \mathrm{K}_{6}=\int_{0}^{\mathrm{a}} \exp \left(\frac{-2 \mathrm{z}}{\mathrm{dp}}\right) \cdot \sum_{\mathrm{n}=1}^{\infty}\left\{\mathrm{n}^{2} \cdot \sin \left(\frac{\mathrm{n} \pi \mathrm{b}}{\mathrm{a}}\right) \cdot \sin \left(\frac{\mathrm{n} \pi \mathrm{z}}{\mathrm{a}}\right) \cdot \exp \left(\frac{-\mathrm{n}^{2} \pi^{2} \mathrm{Dt}}{\mathrm{a}^{2}}\right)\right\} \cdot d \mathrm{z} \\
& \frac{A(t)-A_{0}}{A_{\infty}-A_{0}}=1-\left(\frac{\left(\frac{2}{\pi}\right) \cdot K_{2}-\left(\frac{\pi \cdot d p^{2}}{2 a^{2}}\right) \cdot K_{5}+\left(\frac{\pi^{2} \cdot d p^{2}}{2 a^{3}}\right) \cdot K_{6}}{\text { deno min ator }}\right)
\end{aligned}
$$

Then 


$$
\text { But }\left(\frac{2}{\pi}\right) \cdot \mathrm{K}_{2}-\left(\frac{\pi \cdot \mathrm{dp}^{2}}{2 \mathrm{a}^{2}}\right) \cdot \mathrm{K}_{5}=\mathrm{K}_{7}
$$

with

$$
\mathrm{K}_{7}=\left(\frac{2}{\pi}\right) \cdot \sum_{\mathrm{n}=1}^{\infty}\left\{\left(\frac{1}{\mathrm{n}}\right) \cdot\left[1-\left(\frac{\mathrm{n} \cdot \pi \cdot \mathrm{dp}}{2 \mathrm{a}}\right)^{2}\right] \cdot \sin \left(\frac{\mathrm{n} \pi \mathrm{b}}{\mathrm{a}}\right) \cdot \exp \left(\frac{-\mathrm{n}^{2} \pi^{2} \mathrm{Dt}}{\mathrm{a}^{2}}\right) \cdot\left[1+(-1)^{\mathrm{n}+1} \cdot \exp \left(\frac{-2 \mathrm{a}}{\mathrm{dp}}\right)\right]\right\}
$$

Then

$$
\frac{A(t)-A_{0}}{A_{\infty}-A_{0}}=1-\left(\frac{K_{7}+\left(\frac{\pi^{2} \cdot d p^{2}}{2 a^{3}}\right) \cdot K_{6}}{\text { denomin ator }}\right)
$$

Integrating $\mathrm{K}_{6}$ by parts we get:

$$
\mathrm{K}_{6}=\left(\frac{\mathrm{dp}}{2}\right) \cdot\left(\frac{\pi}{\mathrm{a}}\right) \cdot \mathrm{K}_{8}
$$

$$
\text { where } \quad K_{8}=\int_{0}^{a} \exp \left(\frac{-2 z}{d p}\right) \cdot \sum_{n=1}^{\infty}\left\{n^{3} \cdot \sin \left(\frac{n \pi b}{a}\right) \cdot \cos \left(\frac{n \pi z}{a}\right) \cdot \exp \left(\frac{-n^{2} \pi^{2} D t}{a^{2}}\right)\right\} \cdot d z
$$

Thus

$$
\frac{A(t)-A_{0}}{A_{\infty}-A_{0}}=1-\left(\frac{K_{7}+\left(\frac{\pi^{3} \cdot d p^{3}}{4 a^{4}}\right) \cdot K_{8}}{\text { denomin ator }}\right)
$$

Continuing integrating by parts we obtain:

$$
\mathrm{K}_{8}=\left(\frac{\mathrm{dp}}{2}\right) \cdot \mathrm{K}_{9}-\left(\frac{\mathrm{dp}}{2}\right) \cdot\left(\frac{\pi}{\mathrm{a}}\right) \cdot \mathrm{K}_{10}
$$


where $K_{9}=\sum_{n=1}^{\infty}\left\{n^{3} \cdot \sin \left(\frac{n \pi b}{a}\right) \cdot \exp \left(\frac{-n^{2} \pi^{2} D t}{a^{2}}\right) \cdot\left[1+(-1)^{n+1} \cdot \exp \left(\frac{-2 a}{d p}\right)\right]\right\}$

and $\quad \mathrm{K}_{10}=\int_{0}^{\mathrm{a}} \exp \left(\frac{-2 \mathrm{z}}{\mathrm{dp}}\right) \cdot \sum_{\mathrm{n}=1}^{\infty}\left\{\mathrm{n}^{4} \cdot \sin \left(\frac{\mathrm{n} \pi \mathrm{b}}{\mathrm{a}}\right) \cdot \sin \left(\frac{\mathrm{n} \pi \mathrm{z}}{\mathrm{a}}\right) \cdot \exp \left(\frac{-\mathrm{n}^{2} \pi^{2} \mathrm{Dt}}{\mathrm{a}^{2}}\right)\right\} \cdot d \mathrm{z}$

Then

$$
\frac{A(t)-A_{0}}{A_{\infty}-A_{0}}=1-\left(\frac{K_{7}+\left(\frac{\pi^{3} \cdot d p^{4}}{8 a^{4}}\right) \cdot K_{9}-\left(\frac{\pi^{4} \cdot d p^{4}}{8 a^{5}}\right) K_{10}}{\text { deno min ator }}\right)
$$

But $\quad \mathrm{K}_{7}+\left(\frac{\mathrm{dp}^{4} \cdot \pi^{3}}{8 \mathrm{a}^{4}}\right) \cdot \mathrm{K}_{9}=\left(\frac{2}{\pi}\right) \cdot \sum_{\mathrm{n}=1}^{\infty}\left\{\mathrm{K}_{11} \cdot \mathrm{K}_{12}\right\}$

where $\mathrm{K}_{11}=\left(\frac{1}{\mathrm{n}}\right) \cdot \sin \left(\frac{\mathrm{n} \pi \mathrm{b}}{\mathrm{a}}\right) \cdot \exp \left(\frac{-\mathrm{n}^{2} \pi^{2} \mathrm{Dt}}{\mathrm{a}^{2}}\right) \cdot\left[1+(-1)^{\mathrm{n}+1} \cdot \exp \left(\frac{-2 \mathrm{a}}{\mathrm{dp}}\right)\right]$

and $\quad \mathrm{K}_{12}=1-\left(\frac{\mathrm{n} \cdot \pi \cdot \mathrm{dp}}{2 \mathrm{a}}\right)^{2}+\left(\frac{\mathrm{n} \cdot \pi \cdot \mathrm{dp}}{2 \mathrm{a}}\right)^{4}$

Then

$$
\frac{A(t)-A_{0}}{A_{\infty}-A_{0}}=1-\left(\frac{\left(\frac{2}{\pi}\right) \cdot \sum_{n=1}^{\infty}\left\{K_{11} \cdot K_{12}\right\}-\left(\frac{\pi^{4} \cdot d p^{4}}{8 a^{5}}\right) K_{10}}{\text { deno min ator }}\right)
$$

If we continue integrating by parts, $\mathrm{K}_{12}$ will become:

$$
\mathrm{K}_{13}=1-\left(\frac{\mathrm{n} \cdot \pi \cdot \mathrm{dp}}{2 \mathrm{a}}\right)^{2}+\left(\frac{\mathrm{n} \cdot \pi \cdot \mathrm{dp}}{2 \mathrm{a}}\right)^{4}-\left(\frac{\mathrm{n} \cdot \pi \cdot \mathrm{dp}}{2 \mathrm{a}}\right)^{6}+\left(\frac{\mathrm{n} \cdot \pi \cdot \mathrm{dp}}{2 \mathrm{a}}\right)^{8}-\ldots
$$


This is a geometric series with 1 for first term and $-\left[\left(\frac{\mathrm{n} \cdot \pi \cdot \mathrm{dp}}{2 \mathrm{a}}\right)^{2}\right]$ for the multiplication factor.

The expression of $\mathrm{K}_{13}$ is thus equivalent to:

$$
\mathrm{K}_{13}=1 *\left[\frac{1-\left(-\left(\frac{\mathrm{n} \cdot \pi \cdot \mathrm{dp}}{2 \mathrm{a}}\right)^{2}\right)^{\mathrm{k}}}{1-\left(-\left(\frac{\mathrm{n} \cdot \pi \cdot \mathrm{dp}}{2 \mathrm{a}}\right)^{2}\right)}\right]=\left[\frac{1-\left[(-1)^{\mathrm{k}} \cdot\left(\frac{\mathrm{n} \cdot \pi \cdot \mathrm{dp}}{2 \mathrm{a}}\right)^{2 \mathrm{k}}\right]}{1+\left(\frac{\mathrm{n} \cdot \pi \cdot \mathrm{dp}}{2 \mathrm{a}}\right)^{2}}\right]
$$

where $\mathrm{k}$ is the number of terms in the summation and is infinite

Then

$$
\frac{A(t)-A_{0}}{A_{\infty}-A_{0}}=1-\left(\frac{\left(\frac{2}{\pi}\right) \cdot \sum_{n=1}^{\infty}\left\{K_{11} \cdot K_{13}\right\}+\text { int egral }}{\text { deno min ator }}\right)
$$

Neglecting the integral that remains, assuming that $\left(\frac{\mathrm{n} \cdot \pi \cdot \mathrm{dp}}{2 \mathrm{a}}\right)<<1$ for small $\mathrm{n}$, and noticing that $\left(\frac{1}{n}\right) \cdot \exp \left(\frac{-n^{2} \pi^{2} \mathrm{Dt}}{\mathrm{a}^{2}}\right) \cdot\left[-(-1)^{\mathrm{k}} \cdot\left(\frac{\mathrm{n} \cdot \pi \cdot \mathrm{dp}}{2 \mathrm{a}}\right)^{2 \mathrm{k}}\right]$ becomes zero when $\mathrm{n}$ is great, we finally get the following model [20] for the Fickian diffusion of our system: 


$$
\frac{A(t)-A_{0}}{A_{\infty}-A_{0}}=1-\left\{\left[\frac{\frac{2}{\pi} \cdot \sum_{n=1}^{\infty}\left(\frac{1}{n}\right) \cdot \sin \left(\frac{n \pi b}{a}\right) \cdot \exp \left(\frac{-n^{2} \pi^{2} D t}{a^{2}}\right) \cdot\left(\frac{1+(-1)^{n+1} \cdot \exp \left(\frac{-2 a}{d_{p}}\right)}{1+\left(\frac{n \pi d_{p}}{2 a}\right)^{2}}\right)}{1-\exp \left(\frac{-2 b}{d_{p}}\right)-\frac{b}{a}\left[1-\exp \left(\frac{-2 a}{d_{p}}\right)\right]}\right]\right\}
$$

where $\mathrm{A}(\mathrm{t})$ is the absorbance at any time

$\mathrm{A}_{\mathrm{o}}$ is the initial absorbance

$A_{\propto}$ is the equilibrium absorbance at infinite time

$\mathrm{t}$ is the time

$\mathrm{dp}$ is the penetration depth

$\mathrm{D}$ is the diffusion coefficient

$\mathrm{b}$ is the thickness of the polymer

$\mathrm{a}$ is the total thickness of the polymer and the monomer

$\mathrm{n}$ is the index of summation

Thus we see that the absorbance data can be related to the diffusion coefficient $\mathrm{D}$, which is the only unknown parameter on the right hand side. From the knowledge of the diffusion coefficient, it will be possible to substitute its value in the Fickian model (equation (2.4.o)) to get the concentration profile of the polymer.

Van Alsten and Lustig [20] presented the above equation, (2.4.s), without the assumptions. However, while this equation applies only to the polymer, it was used by Van Alsten and Lustig to measure the uptake of a component diffusing into a polymer, not 
realizing that this equation was specific to the polymer in contact with the ATR crystal. However, it will be shown below that this equation is also valid in modeling the monomer diffusion.

According to the equation (2.4.n), we have:

$$
\mathrm{Cm}(\mathrm{z}, \mathrm{t})=\mathrm{C}_{\mathrm{mo}} .\left(1-\left(\frac{\mathrm{C}(\mathrm{z}, \mathrm{t})}{\mathrm{C}_{\mathrm{o}}}\right)\right)
$$

where $C_{m}(z, t)$ is the concentration profile of the monomer

$\mathrm{C}(\mathrm{z}, \mathrm{t})$ is the concentration profile of the polymer

$\mathrm{C}_{\mathrm{mo}}$ is the initial concentration of the monomer

$\mathrm{C}_{\mathrm{o}}$ is the initial concentration of the polymer

This equation does not take into consideration the density changes and volume changes upon mixing.

Substituting (2.4.n) into (2.4.1) we get the expression of the absorbance of the monomer at any time:

$$
A(t)=\int_{0}^{a} \alpha \cdot S \cdot \exp \left(\frac{-2 z}{d p}\right) \cdot C_{m o}(z, t) \cdot\left\{1-\left(\frac{b}{a}\right)-\frac{2}{\pi} \sum_{n=1}^{\infty}\left\{\left(\frac{1}{n}\right) \cdot \sin \left(\frac{n \pi b}{a}\right) \cdot \cos \left(\frac{n \pi z}{a}\right) \cdot \exp \left(\frac{-n^{2} \pi^{2} D t}{a^{2}}\right)\right\}\right\} d z
$$

The expression of the initial and final absorbances of the monomer are:

$$
\mathrm{A}_{0}=\int_{0}^{\mathrm{b}} \alpha \cdot \mathrm{C}_{\mathrm{mo}}(\mathrm{z}, 0) \operatorname{S} \cdot \exp \left(\frac{-2 \mathrm{z}}{\mathrm{dp}}\right) \cdot \mathrm{dz}+\int_{\mathrm{b}}^{\mathrm{a}} \alpha \cdot \mathrm{C}_{\mathrm{mo}} \cdot(\mathrm{z}, 0) \operatorname{S} \cdot \exp \left(\frac{-2 \mathrm{z}}{\mathrm{dp}}\right) \cdot \mathrm{dz}
$$




$$
\begin{aligned}
& =\alpha \cdot C \text { mo.S. } \int_{b}^{a} \exp \left(\frac{-2 z}{d p}\right) \cdot d z \\
& =\alpha \cdot C_{\text {mo.S }} \cdot\left(\frac{d p}{2}\right) \cdot\left(\exp \left(\frac{-2 b}{d p}\right)-\exp \left(\frac{-2 a}{d p}\right)\right) \\
& A_{\infty}=\int_{0}^{a} \alpha \cdot S \cdot \exp \left(\frac{-2 z}{d p}\right) \cdot C_{m}(z, \infty) \cdot d z \\
& \text { but } \mathrm{C}_{\mathrm{m}}(\mathrm{z}, \infty)=\left(\mathrm{C}_{\mathrm{m} 0}\right) \cdot\left(1-\left(\frac{\mathrm{b}}{\mathrm{a}}\right)\right) \text { since } \exp \left(\frac{-n^{2} \pi^{2} D t}{a^{2}}\right)=0 \\
& \mathrm{~A}_{\infty}=\alpha \cdot \mathrm{C}_{\mathrm{m} 0} \cdot \mathrm{S} \cdot\left(1-\frac{\mathrm{b}}{\mathrm{a}}\right) \cdot \int_{0}^{\mathrm{a}} \exp \left(\frac{-2 \mathrm{z}}{\mathrm{dp}}\right) \cdot \mathrm{dz} \\
& =\alpha \cdot C_{m 0} \cdot S \cdot\left(1-\frac{b}{a}\right) \cdot\left(\frac{d p}{2}\right) \cdot\left(1-\exp \left(\frac{-2 a}{d p}\right)\right) \\
& \left(\frac{A_{\infty}}{A_{o}}\right)=\frac{\left[\left(1-\frac{b}{a}\right)\left(1-\exp \left(\frac{-2 a}{d p}\right)\right)\right]}{\left[\exp \left(\frac{-2 b}{d p}\right)-\exp \left(\frac{-2 a}{d p}\right)\right]} \\
& \mathrm{A}_{\infty}-\mathrm{A}_{\circ}=\alpha . \mathrm{C}_{\mathrm{mo}} . \mathrm{S} .\left(\frac{\mathrm{dp}}{2}\right) .(\text { deno min ator }) \\
& \text { where denominator }=\left[\left(1-\exp \left(\frac{-2 b}{d p}\right)\right)-\left(\frac{b}{a}\right) \cdot\left(1-\exp \left(\frac{-2 a}{d p}\right)\right)\right]
\end{aligned}
$$

We notice that $A_{t}=A_{\infty}+\alpha \cdot C_{m o} \cdot S \cdot\left(-\frac{2}{\pi}\right) \cdot K_{1}$ 


$$
\text { where } \quad K_{1}=\int_{0}^{a} \exp \left(\frac{-2 z}{d p}\right) \cdot \sum_{n=1}^{\infty}\left\{\left(\frac{1}{n}\right) \cdot \sin \left(\frac{n \pi b}{a}\right) \cdot \cos \left(\frac{n \pi z}{a}\right) \cdot \exp \left(\frac{-n^{2} \pi^{2} D t}{a^{2}}\right)\right\} \cdot d z
$$

Thus we get

$$
\frac{\mathrm{A}(\mathrm{t})-\mathrm{A}_{0}}{\mathrm{~A}_{\infty}-\mathrm{A}_{0}}=1+\left(\frac{\left(-\frac{2}{\pi}\right) \cdot \mathrm{K}_{1}}{\left(\frac{\mathrm{dp}}{2}\right) \cdot(\text { deno min ator })}\right)
$$

The above equation is equivalent to what we have derived for the polymer and therefore the equation (2.4.s) is valid for both components under the assumptions previously outlined.

\subsection{4 - DETERMINATION OF THE DIFFUSION COEFFICIENTS BY ATR SPECTROSCOPY}

Many authors have focused their attention on the diffusion behavior of small molecules in polymers $[119,120,121,122,125,126,127,128,129,130,131]$. This is discussed in the first part of this subsection. In the past years, several studies have also been concerned with the diffusion of polymers within polymers $[20,21,22,23,24,25,26$, 74], as presented in the second part of this subsection.

\subsubsection{1 - Small Molecule Diffusion into Polymers}

ATR measurements of small molecule diffusion have been carried out by placing the polymer in contact with a circulating fluid in order to keep a constant concentration at the surface of the polymer. Since the initial and boundary conditions are different from the ones we have assumed in Subsection 2.4.3.3, equation (2.4.s) is not valid in this case. The appropriate corresponding expression can be found in the literature [125], assuming also a one-dimensional Fickian diffusion with a constant diffusion coefficient. 
Some investigators have focused on the influence of the polymer morphology on the diffusion. Furlan [127] studied the diffusion of n-decyl alcohol into hydrogenated polybutadienes and underlined the influence of polymer morphology on the diffusion, especially the importance of crystallinity on transport. Indeed, diffusion through an amorphous material is significantly greater than in a crystalline material. Van Alsten and Coburn [121] studied the influence of cure conditions and backbone chemistry of polyimides on the diffusion of heavy water. As the chain backbone stiffness increased, and also as the density of the amorphous phase increased, for a given backbone composition, the diffusivity decreased. Among crystallinity, backbone orientation and density of the glass, the latter was found to be the most critical factor.

FTIR-ATR experiments were found to be accurate compared to other techniques. Fieldson and Barbari [125] measured the diffusion of water in polyacrylonitrile below and above the glass transition temperature of the polymer and found a good agreement with the values reported in the literature employing other techniques. Aging behavior was reported: the higher the temperature treatment of the polymer, the greater the diffusion coefficient, due to the loss of residual solvent incorporated during film casting. Good agreement between gravimetric sorption, nuclear magnetic resonance and ATR measurements [120] was found for the acetone-polypropylene, methanol-polystyrene and methanol-poly(methyl methacrylate) systems. A model for case II diffusion was also developed. Farinas et al. [126] analyzed the diffusion of urea into a silicone polymer and showed that the results were consistent with bulk transport techniques. Semwal et al. [128] also reported a good agreement between the ATR method and weight gain methods for the diffusion of sulfur mustard and oxygen mustard in polypropylene and biaxialoriented polypropylene. Hong et al. [129] measured the diffusion of methyl ethyl ketone in polyisobutylene by vapor sorption FTIR-ATR spectroscopy and underlined that the method led to coherent results, compared to a conventional gravimetric sorption balance.

Other investigators who have successfully employed the ATR method, include: Skourlis and McCullough [119] who have studied the diffusion of liquid diamine into a thermoset polymer (epoxy) over a wide temperature range; Immordino et al. [122, 130] who have studied the diffusion of both epoxy and amine prepolymers in polysulfone and 
incorporated the curing kinetics in the diffusion model and; Kwan and Ward [131] who have tried to correlate penetrant diffusion in an epoxy laminate with the solubility parameters and the molecular size of diverse penetrants.

\subsubsection{2 - Interdiffusion of Polymers}

When studying the diffusion of polymers within polymers, the schematic of the polymeric system is exactly the same as the one previously illustrated in Figure 2.7, except that a polymer and not a monomer is used as a second layer on the ATR crystal.

Van Alsten and Lustig derived the equations for measuring mutual diffusion coefficients of polymers in melts, provided that the diffusion behavior was totally Fickian. Results for polystyrene and poly(methyl methacrylate) were then presented [20]. The influence of crystallinity on interdiffusion was also studied; for example as in the diffusion of amorphous poly(ether imide) into amorphous and semicrystalline poly(aryl ether ketone ketone) [22] and the diffusion of atactic polystyrene-d into atactic and semicrystalline isotactic polystyrene [23]. It was reported that crystallization retarded transport and a non-Fickian behavior for semicrystalline polymers at low temperature and high penetrant molecular weight was observed.

Jabbari and Peppas concentrated their analysis on interdiffusion of polystyrene (PS) and poly(vinyl methyl ether) (PVME) [21, 24, 26]. It was found that after contact was established between the two polymers, the faster diffusing component swelled the slower diffusing component prior to interdiffusion across the interface, therefore, in good agreement with the fast theory of diffusion previously mentioned. The results were analyzed with a combination of Fickian and case II models at temperatures just below and above the glass transition temperature. The lower the temperature, the greater the percent of non-Fickian behavior. It was the first time that case II diffusion was observed or treated as such above the entanglement molecular weight and for below and above the glass transition temperature of the slower diffusing component. The effects of the molecular weight, the polydispersity, and the temperature were evaluated. The diffusion coefficient was independent of PS molecular weight but strongly depended on the PVME molecular 
weight, while polydispersity seriously affected the diffusion. Comparison of this system with the system consisting of polystyrene and poly(isobutyl vinyl ether) [25] was also carried out. The first system involved compatible polymers whereas the second system consisted of incompatible polymers. It was shown that the compatibility between the two polymers controlled the extent of interdiffusion.

Rajagopalan et al. [74] studied the diffusion at the epoxy/amorphous poly(aryl ether ether ketone) interface. In this case, an equation for sorption was used, in spite of the fact that such an equation is essentially used to model changes of mass with time. It is based upon certain limiting constraints [102] and is less accurate than the diffusion model developed in Subsection 2.4.3.

\section{5 - ADVANTAGES AND LIMITATIONS OF ATR SPECTROSCOPY}

This section discusses the main advantages and limitations associated with the ATR technique.

\subsection{1 - ADVANTAGES}

The ATR method is attractive for several reasons:

- The data are collected in situ and in real time.

- It is a nondestructive technique. The samples are not damaged by the use of this technique, and they can be used later for other kinds of analysis. In fact, a physical contact is just required; there is no need for dissolving, labeling or thermally fragmenting the material and therefore the sampling error is reduced.

- It is possible to work with small penetration depths (from 0.1 to $10 \mu \mathrm{m}[112,119]$ ).

- It is applicable to solids, liquids, and thin films, provided that the different components have infrared distinguishable absorption bands. 
- It is an excellent technique for polymer films because the thickness of the film is not important.

- Co-current chemical reactions or physical interactions, such as hydrogen bonding, can be monitored by studying changes in the infrared spectrum.

- Any change on the surface due to diffusion can be observed and the diffusion coefficients can be measured in a wide range, from $10^{-5}$ to $10^{-16} \mathrm{~cm}^{2} \cdot \mathrm{s}^{-1}$ [19].

- The equipment, whose cost is reasonable, is quite simple to use and does not require vacuum.

\subsection{2 - LIMITATIONS}

However ATR spectroscopy presents some limitations:

- It is not possible to see more than $10 \mu \mathrm{m}$ of the sample, which is a problem when the chemical composition of the surface is different from that of the bulk or if the interphase areas are large.

- A good contact between the sample and the ATR crystal, especially right in the center, is necessary to ensure that the evanescent wave penetrates into the sample.

- Although the composition profile is calculated and not measured directly, it is still a pretty good estimate, assuming that the diffusion is well-described by a Fickian model.

- It is difficult to have reproductible (crystal/sample) contact. Positioning the ATR crystal in the sampling accessory, keeping the crystal clean, flat, smooth, and scratch free, and putting a constant and uniform pressure on the sample are necessary in order to keep the penetration depth constant. 


\section{CHAPTER 3}

\section{EXPERIMENTAL}

As mentioned in the introduction of this thesis, mechanical properties of composites can be greatly improved by using an intermediate sizing material between carbon fiber and thermoset resin. Poly(vinyl pyrrolidone) (PVP) is being used as a sizing material for composites composed of carbon fiber and vinyl ester matrix. Since the extent of mutual diffusion between the PVP and the vinyl ester matrix plays a critical role in determining the mechanical properties of the composite, the molecular diffusion across the (PVP/vinyl ester matrix) interface has to be determined. The diffusion between PVP and vinyl ester monomer (VE) was studied in this research by FTIR-ATR spectroscopy. The present chapter provides some information concerning the experimental details of this study.

The first section of this chapter focuses on the experimental procedure for the sample preparation. The equipment used in this research is then presented. The third section explains how to generate infrared spectra. The spectral manipulations which can be performed are discussed in the final section.

\section{1 - SAMPLE PREPARATION}

The proper choice of the ATR crystal is essential to a successful experiment. This section starts with describing the ATR crystal used in this research, followed by detailed characteristics of the PVP and the VE materials used. Parameters, such as the refractive indices of the materials and the angle of incidence chosen in the experiments, are given before detailing the preparation of the (PVP/VE) sample. 


\subsection{1 - CHOICE OF THE INTERNAL REFLECTION ELEMENT (IRE) OR ATR CRYSTAL}

Several requirements have to be considered in the choice of the IRE: refractive index, spectral range, price, surface quality, toughness, chemical inertness, temperature, toxicity, etc. Table 3.1 summarizes the information found in the literature $[110,112,113]$ for zinc selenide $(\mathrm{ZnSe})$ and silicon $(\mathrm{Si})$ crystals that were bought from Harrick Scientific Corporation.

Table 3.1: Characteristics of the ZnSe and Si crystals

\begin{tabular}{|l|r|r|}
\hline material for ATR & ZnSe (or Irtran-4) & Si \\
\hline color & yellow & grey \\
\hline refractive index $\mathrm{n}_{1}$ & 2.42 & 3.42 \\
\hline useful transmission range $\left(\mathrm{cm}^{-1}\right)$ & $20,000-700$ & $9500-1500$ \\
\hline hardness (psi) & 150 & 1,150 \\
\hline chemical properties & 1788 & 1683 \\
\hline melting point $(\mathrm{K})$ & soluble in strong acids & soluble in HF and $\mathrm{HNO}_{3}$ \\
\hline thermal conductivity $\left(\mathrm{cgs} \times 10^{-2}\right)$ & 3.1 at $327 \mathrm{~K}$ & 39 at $313 \mathrm{~K}$ \\
\hline linear thermal expansion $\left(\mathrm{x} 10^{-6}\right)$ & 8 at $300 \mathrm{~K}$ & 3.9 at $250 \mathrm{~K}$ \\
\hline price $($ Harrick Scientific Corporation) & & $\$ 825$ \\
\hline hemisphere & & $\$ 850$ \\
\hline
\end{tabular}

Note that Irtran is a registered trade mark of the Eastman Kodak Co.

$\mathrm{ZnSe}$ is insoluble in water and most organic solvents but dissolves in strong acids. The surface becomes etched during prolonged exposure to strong acids and alkalies. Because of the ionic sites on the surface of the material, there is adsorption of polar and ionic species. With other oxidizing agents there is formation of selenium dioxide [113]. An important fact is that sometimes the spectra obtained with $\mathrm{ZnSe}$ may present an impurity band at $1100 \mathrm{~cm}^{-1}$. But the main disadvantage of $\mathrm{ZnSe}$ is that it cuts off at about $600 \mathrm{~cm}^{-1}$, masking a small part of the mid-infrared region. $\mathrm{Si}$ is not affected by mild acids, is insoluble in water and organic solvents, but soluble in alkalies. The absorbance of silicon decreases considerably with increasing temperature [110], therefore it is usable up to $300^{\circ} \mathrm{C}$ only. 
$\mathrm{ZnSe}$ was chosen as the IRE since it was planned to work in the mid-infrared spectral region (from $4,400 \mathrm{~cm}^{-1}$ to $400 \mathrm{~cm}^{-1}$ ). The molecular weight of the crystal used was $144.34 \mathrm{~g} / \mathrm{mol}$ and its density $5.27 \mathrm{~g} / \mathrm{cm}^{3}$. A Seagull ${ }^{\mathrm{TM}}-\mathrm{ATR}$ attachment bought from Harrick Scientific Corporation was used, requiring a hemisphere, a single reflection IRE, whose diameter was $25 \mathrm{~mm}$ and height $12.5 \mathrm{~mm}$. The hemisphere had a plane and polished reflecting surface.

\subsection{2 - THE MATERIALS}

The materials used in this study were poly(vinyl pyrrolidone) (PVP) and vinyl ester monomer (VE). The PVP K90 was obtained from BASF Corporation, while the vinyl ester monomer was supplied by Dow Chemical Co. Their characteristics are given below.

\subsubsection{1 - The Poly (Vinyl Pyrrolidone) PVP K90, or PVP}

Other chemical names for the PVP can be found in the literature: they are poly(Nvinyl pyrrolidone) or poly(1-vinyl-2-pyrrolidone). The PVP is an amorphous thermoplastic and a brittle material. It has been used for various purposes [132, 133], such as an additive in ultrafiltration membranes, as a stabilizer in suspension polymerization, as a thickener in the cosmetic or food industries, as a coating aid in the photographic industry, and more recently as a sizing material for fiber reinforced composites. The molecular weight of the PVP repeat unit is equal to $111 \mathrm{~g} \cdot \mathrm{mol}^{-1}$. The chemical structure of the PVP repeat unit is given in Figure 3.1. At this point, it is interesting to point out some infrared sensitive groups, such as the carbonyl group $\mathrm{C}=\mathrm{O}$ and the $\mathrm{N}-\mathrm{C}$ vibration group. The PVP is soluble in water and in organic liquids. Table 3.2 gives some information about its solubility in various solvents. 
Table 3.2: Solubility of PVP in various solvents (from [132])

\begin{tabular}{|c|c|}
\hline SOLUBLE & INSOLUBLE \\
\hline $\begin{array}{l}\begin{array}{l}\text { water } \\
\text { alcohols } \\
\\
\text { methanol } \\
\text { ethanol } \\
\text { propanol } \\
\text { butanol }\end{array} \\
\text { acids } \\
\\
\text { formic acid } \\
\text { acetic acid } \\
\text { propionic acid } \\
\text { esters } \\
\text { ethyl lactate } \\
\text { ketones } \\
\text { methyl cyclohexanone } \\
\text { chlorinated hydrocarbons } \\
\text { methylene dichloride } \\
\text { chloroform } \\
\text { ethylene dichloride } \\
\text { amines } \\
\text { ethylene diamine } \\
\text { triethanolamine } \\
\text { glycerol } \\
\text { glycols } \\
\text { diethylene glycol } \\
\text { polyethylene glycol } 400 \\
\text { lactams } \\
\text { nitroparaffins }\end{array}$ & 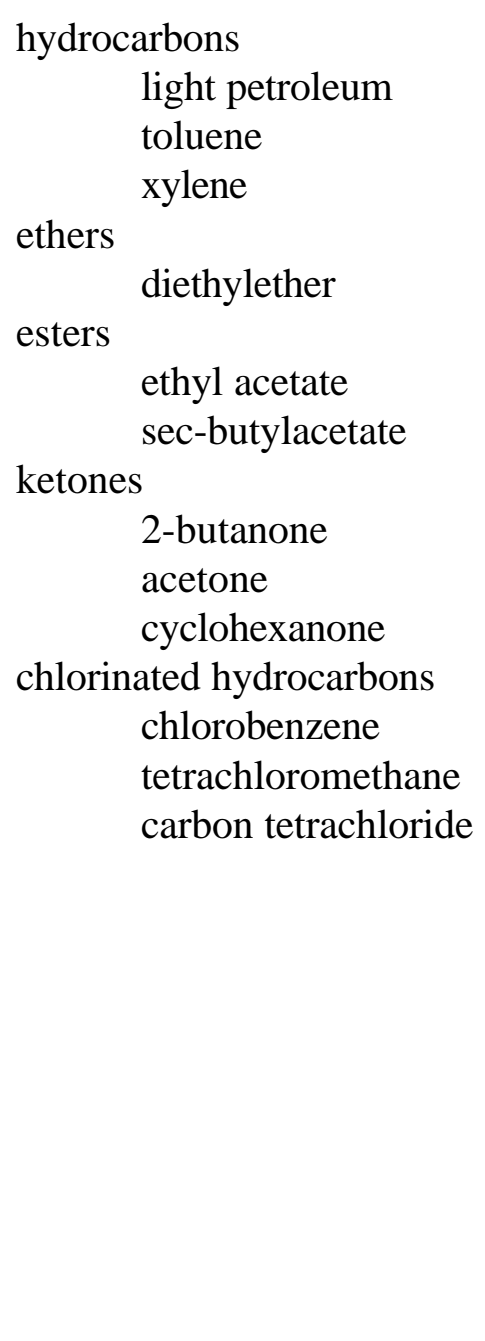 \\
\hline
\end{tabular}




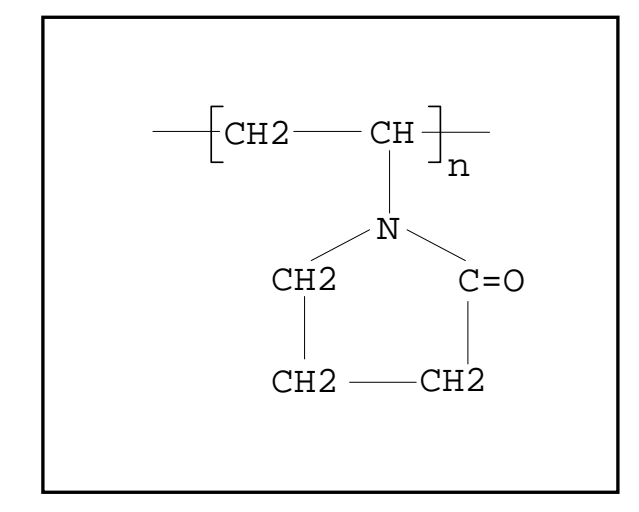

Figure 3.1: Repeat unit of poly(vinyl pyrrolidone)

PVP can be obtained at different molecular weights, which are distinguished by a K number. For example, PVP K90 was used in this research. The K-value is usually determined at $1 \% \mathrm{wt} / \mathrm{vol}$ of a given PVP sample in an aqueous solution. Its expression [132] is derived from the Fikentscher's equation:

$$
\log \left(\frac{\eta_{\mathrm{rel}}}{\mathrm{C}}\right)=\left(\frac{75 \mathrm{~K}_{\mathrm{o}}{ }^{2}}{1+1.5 \mathrm{~K}_{\mathrm{o} . \mathrm{C}}}\right)+\mathrm{K}_{\mathrm{o}}
$$

$$
\begin{aligned}
& \text { where } \mathrm{K}=1000 \mathrm{~K}_{\mathrm{o}} \\
& \qquad \begin{aligned}
\eta_{\text {rel }} & =\text { relative viscosity } \\
\mathrm{C} & =\text { concentration of the solution in } \mathrm{g} / 100 \mathrm{ml}
\end{aligned}
\end{aligned}
$$

(3.1.a) is equivalent to:

$$
K=\left(\frac{\sqrt{300 C \log \left(\eta_{r e l}\right)+\left(C+1.5 C \log \left(\eta_{r e l}\right)\right)^{2}}+1.5 C \log \left(\eta_{r e l}-C\right)}{0.15 C+0.003 C^{2}}\right)
$$


The intrinsic viscosity $[\eta]$ is defined as:

$$
[\eta]=\lim _{\mathrm{C} \rightarrow 0}\left(\frac{\ln \left(\eta_{\text {rel }}\right)}{C}\right)
$$

Then the viscosity average molecular weight $\mathrm{M}_{\mathrm{v}}$ is calculated from the Mark-Houwink equation [134]:

$$
[\eta]=\mathrm{k} \cdot \mathrm{M}_{\mathrm{v}}{ }^{\mathrm{a}}
$$

where $\mathrm{k}$ and a are constant for a particular polymer-solvent pair at a particular temperature

The properties of the materials, as the $T_{\mathrm{g}}$, vary according to the average molecular weight. The $T_{g}$ of linear PVP [133] for instance varies according to the equation:

$$
\mathrm{Tg}\left({ }^{\circ} \mathrm{C}\right)=175-\left(\frac{9685}{\mathrm{~K}^{2}}\right)
$$

The white powder PVP K90 had a viscosity-average molecular weight $M_{v}$ of $1,100,000 \mathrm{~g} / \mathrm{mol}$ and a glass transition temperature $\mathrm{T}_{\mathrm{g}}$ of $178^{\circ} \mathrm{C}$ [95].

\subsubsection{2 - The Vinyl Ester Monomer, or VE}

The structure of the VE is shown in Figure 3.2. The infrared sensitive functional groups of the VE are the carbonyl groups $\mathrm{C}=\mathrm{O}$, the alcohol groups $\mathrm{OH}$ and the benzene rings. 


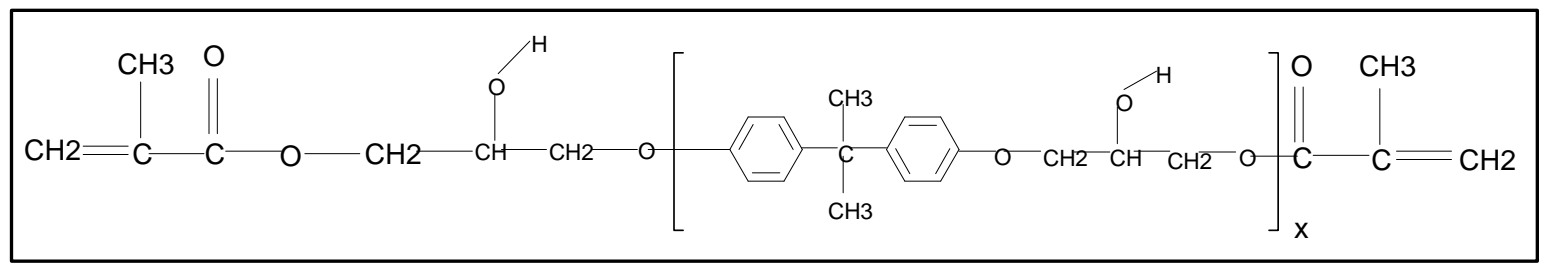

Figure 3.2: Structure of the vinyl ester monomer

The vinyl ester oligomer had a number average molecular weight equal to $690 \mathrm{~g} / \mathrm{mol}$ ("x" was thus equal to 1.65), and was not diluted with styrene. However, it contained an inhibitor, 1,4-Benzoquinone, whose structure is shown in Figure 3.3, and which produced a pale straw color. The inhibitor was added in order to prevent eventual gelation at ambient temperature. This inhibitor reacts with the unavoidable free radical of the vinyl ester monomer and thus avoids polymerization.

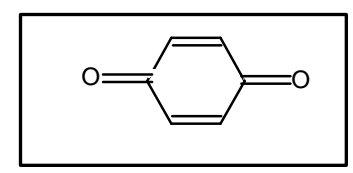

Figure 3.3: Structure of 1,4-Benzoquinone

The VE was a transparent solid at the temperature of $5^{\circ} \mathrm{C}$, and therefore had to be heated to a temperature of about $50^{\circ} \mathrm{C}$ in order to take a sample. This temperature was assumed not to modify the VE. It should be noted that the VE became sticky after some time at room temperature; thus its $\mathrm{T}_{\mathrm{g}}$ was probably between $5^{\circ} \mathrm{C}$ and $25^{\circ} \mathrm{C}$.

\subsection{3 - DETERMINATION OF PARAMETERS}

In order to carry out ATR measurements, parameters such as the refractive indices of the materials and the critical angle have to be determined. The refractive index of PVP is 
1.53 as listed in the polymer handbook [135]. The refractive index of the $\mathrm{ZnSe}$ crystal is $2.42[110,112,113,114]$. The critical angle, $\theta c$, was calculated by the use of the equation (2.3.b). The critical angle was equal to $39.21^{\circ}$ for the given refractive indices. Since the angle of measurement cannot be too close to the critical angle, the angle of incidence was chosen as $45^{\circ}$. Therefore, as the spectra were free of distortions, the band heights could be used for quantitative analysis. The same IRE crystal and an angle of incidence of $45^{\circ}$ were kept for all the experiments.

\subsection{4 - PREPARATION OF THE SAMPLE FOR THE PVP-VE SYSTEM}

The thin film of PVP was cast directly from solution in order to develop a good contact between the polymer and the ATR crystal. The following procedure was used. Since the PVP was soluble in water, a small amount of the white dried PVP was dissolved in water at a concentration of $5.885 \%$ by weight. The system was stirred 2 hours to insure complete mixing. Using a micropipette, a drop of the liquid PVP was placed on the crystal layed horizontally. This drop was left about 6 hours at room temperature in order to allow the water to evaporate. The film of PVP was clear, transparent, glassy and hard. Then the thickness of the film was measured via profilometry [136]. Briefly, the profilometry technique gives the map of the surface via a stylus, which makes repeated and parallel traces over the surface and records the contour lines. The thickness of the PVP was typically on the order of $3 \mu \mathrm{m}$.

A thick film of the monomer was put on an aluminium foil in order to avoid contact directly with the heater. Its thickness was measured with the naked eye and was about $0.5 \mathrm{~mm}$.

Then the thin PVP film was pressed against the thick monomer layer. The area of the PVP was always larger than that of the VE in order to protect the ATR crystal from being exposed to and damaged by the VE.

The system shown in Figure 3.4 was contained in the ATR attachment equipped with a heating cell and the experiments started. Initially the interface was located at $\mathrm{z}=\mathrm{b}$, 
which was outside the penetration depth. As the VE migrated into the PVP, the relative concentration of the components changed within the penetration depth.

The FTIR-ATR technique allows one to follow the diffusion process by sampling the concentration changes of the two components with time at a given temperature. The concentration changes are obtained directly from variations in the height of the characteristic absorption bands.

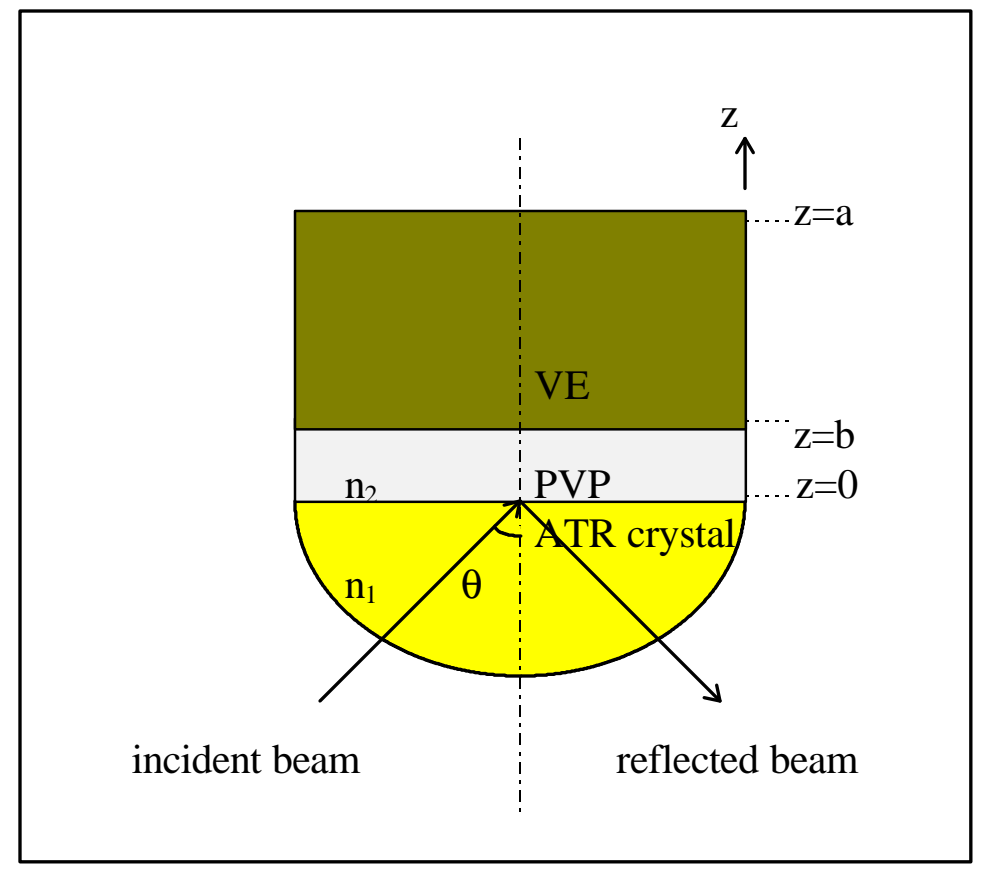

Figure 3.4: Schematic of the ATR sample system

\section{2 - EQUIPMENT}

A description of the temperature controller, the attachment, and the infrared spectrometer used in this investigation is given in this section. 


\subsection{1 - TEMPERATURE CONTROLLER}

The dependence of diffusion on temperature can be evaluated by controlling the temperature of the sample via a heating cell. The sample holder was equipped with a heater. A thermocouple and a heat cartridge $(1 / 4 \times 1.25,100 \mathrm{~W})$ were connected from the heater to a temperature controller, which maintained a constant temperature. An OMEGA® Model CN-2011TC-DC1 programmable controller was used to regulate the given temperature of the experiment within $\pm 0.1^{\circ} \mathrm{C}$, up to a maximum of $200^{\circ} \mathrm{C}$.

The system represented in Figure 3.5, consisting of the ATR crystal, the two samples, the aluminium foil and the heating unit, was sandwiched together and placed in the Seagull ATR attachment.

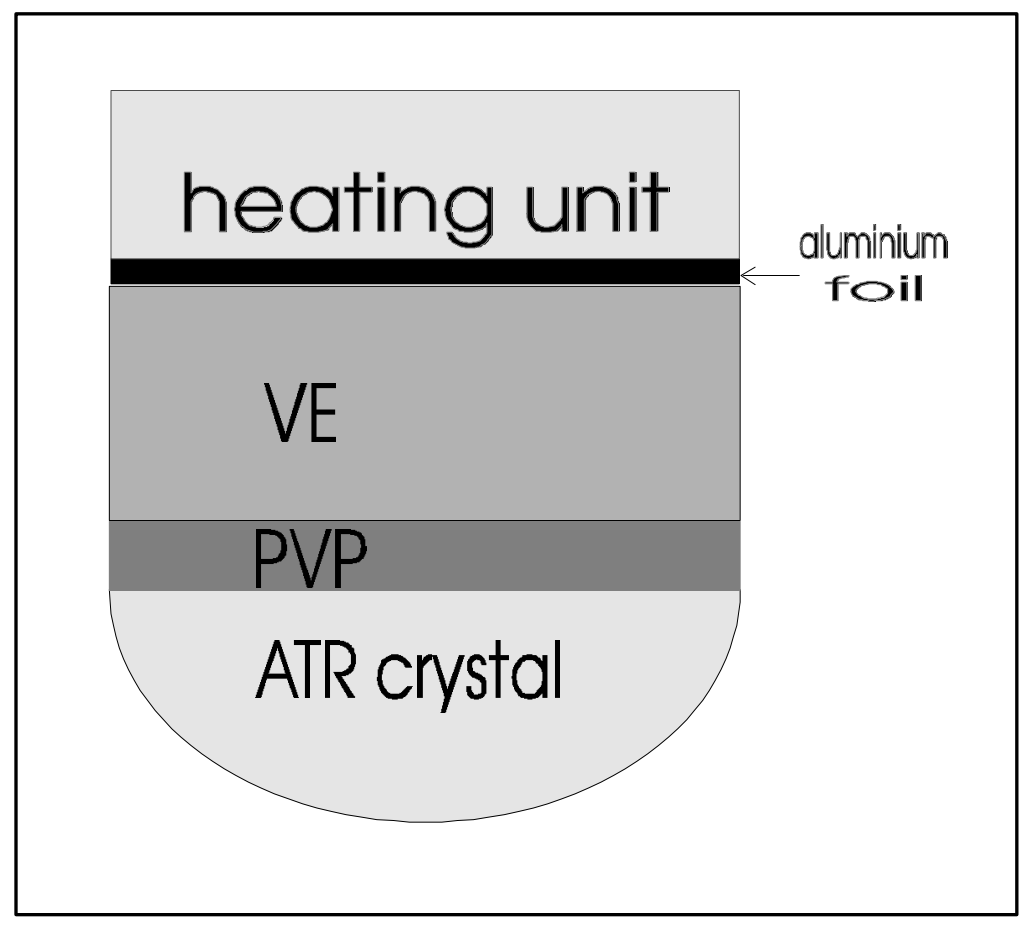

Figure 3.5: Diagram of the ATR cell 


\subsection{2 - ATTACHMENT}

A Seagull ${ }^{\mathrm{TM}}$ ATR attachment allows the sampling of one specific area as a function of the penetration depth. This depth is controlled by the angle of incidence, which can be varied from $5^{\circ}$ ot $85^{\circ}$. In the Seagull ${ }^{\mathrm{TM}}$ attachment, the light is reflected from the source to the IRE by the use of mirrors and then back from the IRE to the detector, as illustrated in Figure 3.6.

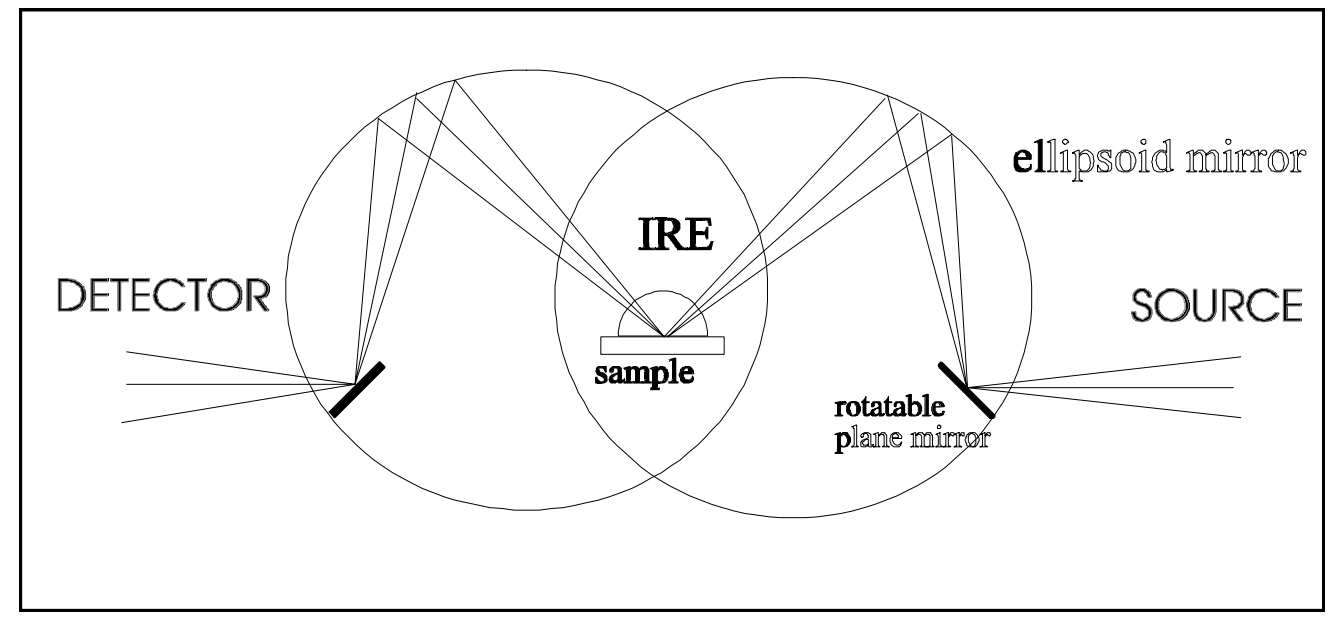

Figure 3.6: Principle of the Seagull ${ }^{\mathrm{TM}}$ attachment (from [137])

\subsection{3 - INFRARED SPECTROMETER}

The infrared spectra were obtained on a BIO-RAD FTS-40A spectrometer purchased from Bio-Rad Laboratories, Inc. The spectrometer is equipped with a liquid nitrogen cooled mercury-cadmium-telluride ( $\mathrm{HgCdTe})$ or "MCT" detector. The detector acts as a transducer, by transforming the infrared intensity into an electrical signal, which is subsequently Fourier transformed into a spectrum. The MCT detector is a semiconductor consisting of an alloy of three elements. The infrared photons which hit the detector cause electrons to be promoted from the valence band to the conduction band, where they can 
respond to an applied voltage. The resulting electrical current is directly proportional to the number of infrared photons and thus to the infrared intensity. MCT detectors are very sensitive and very fast compared to the common deuterated triglycine sulfate (DTGS) detectors. MCT detectors must be cooled with liquid nitrogen in order not to detect noise due to the heat of the detector. Liquid nitrogen has to be added every six hours. The BIORAD FTS-40A spectrometer in our laboratory includes a dynamic alignment in order to erase scattered radiation coming from optical misalignment [110]. The compartment of the spectrometer was gas purged in order to avoid water sorption by the $\mathrm{KBr}$ beamsplitter and to minimize the amount of water vapor and carbon dioxide within the compartment. The spectrometer is connected to a BIO-RAD data station, running a BIO-RAD Laboratories software. The computer converts interferograms into spectra by performing the Fourier transform.

\section{3 - COLLECTING A SPECTRUM}

This section explains how the infrared spectra were performed.

\subsection{1 - SPECTROMETER SET UP}

Only one metal mesh screen and a rail mount of the attachment were inside the compartment for the set up. The sampling parameters used for the set up are given in Table 3.3 .

A scan corresponds to one translation of the moving mirror back and forth. The scan speed selection selects the laser modulation frequency, which controls the velocity of the moving mirror. The delay is the interval of time between the command execution and the first scan. An aperture decreases the effects of angular divergence and thus increases the signal-to-noise ratio (SNR). Angular divergence is a phenomenon which describes the nonparallel nature of the light rays, hence the beam shape is actually a cone. The low pass filter cancels distortion due to noise in the spectrum; its value is always equal to $4.5 \mathrm{kHz}$ for a 
MCT detector. Placing an aperture and a metal mesh screen in the beam eliminates detector saturation which occurs when there is too much infrared radiation striking the detector.

Once the set up program has been run, other commands, such as autoalignment, autoscale, and calibration were performed. Then a $100 \%$ line, which represents the ratio of two consecutive spectra, was run. Ideally this line should be straight at $100 \%$ transmittance, and without noise. Any large curvature indicates beamsplitter misalignment.

Table 3.3: FTIR spectroscopy sampling parameters

\begin{tabular}{|l|l|}
\hline Y-axis & autoscaled \\
\hline detector & sample compartment \\
\hline scan speed selection & $20 \mathrm{kHz}$ \\
\hline delay & $3 \mathrm{~s}$ \\
\hline aperture & $0.5 \mathrm{~cm}^{-1}$ \\
\hline sample beam & not installed \\
\hline low pass filter & $4.5 \mathrm{kHz}$ \\
\hline collect sensitivity & 1 \\
\hline
\end{tabular}

\subsection{2 - SPECTRUM COLLECTION}

The Seagull ${ }^{\mathrm{TM}}$ attachment was placed in the compartment of the spectrometer. The spectrum of the clean ATR crystal without a sample was used as the background, shown in Figure 3.7, thereby accounting for and eliminating any contributions of the instrument and the environment to the spectra. The scanning conditions, outlined in Table 3.4, were assumed to be sufficient to obtain a high signal-to-noise ratio (SNR) and a good resolution to resolve all the bands in the spectrum.

A $4 \mathrm{~cm}^{-1}$ resolution spectrum contains a data point every $4 \mathrm{~cm}^{-1}$. The experiments were done in the mid-IR spectral region, from $4,400 \mathrm{~cm}^{-1}$ to $400 \mathrm{~cm}^{-1}$. For this particular region, the number of data points is: $\left((4000-400) \mathrm{cm}^{-1}\right) /\left(4 \mathrm{~cm}^{-1} /\right.$ data point $)=900$ data points. The number of data points collected depends thus on the resolution. A $4 \mathrm{~cm}^{-1}$ 
spectrum will show many sharp features and is considered a high resolution spectrum. Spectra at high resolution are noisier than those obtained with low resolution. The signalto-noise ratio (SNR) is lower since SNR is directly proportional to the resolution. Each spectrum is the average of a certain number of scans $\mathrm{N}$, in this case 64. SNR is also directly proportional to $(\mathrm{N})^{(1 / 2)}$, while the time spent measuring a spectrum is directly proportional to $\mathrm{N}$. The undersampling ratio (UDR) controlls the frequency of the interferogram sampling; it has to be set to 2 for the mid-IR spectral region.

Table 3.4: FTIR spectroscopy scanning conditions

\begin{tabular}{|l|l|}
\hline spectrum type & background \\
\hline resolution & 4 \\
\hline number of scans & 64 \\
\hline spectral region start & $4,400 \mathrm{~cm}^{-1}$ \\
\hline spectral region end & $400 \mathrm{~cm}^{-1}$ \\
\hline undersampling ratio (UDR) & 2 \\
\hline
\end{tabular}

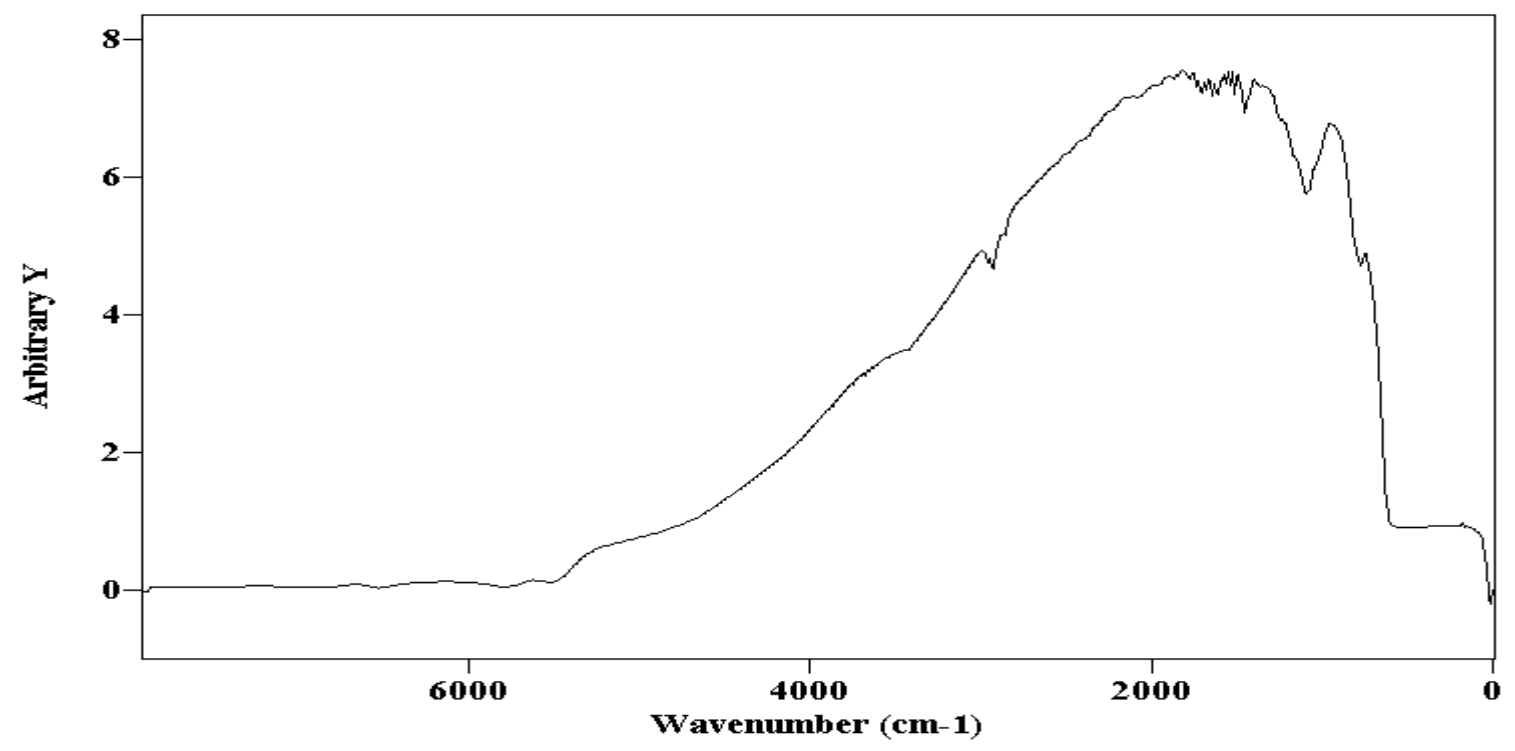

Figure 3.7: A single beam background spectrum of $\mathrm{ZnSe}$ 
Once the background had been collected, the \% absorbance spectrum of the sample could be collected in situ by giving a spectrum name for the spectrum type and by choosing the background file previously stored. The \% transmittance could also be obtained, but quantitative analysis requires absorbance units.

It is extremely important to have very low noise data which are free from water vapor and carbon dioxide. Indeed, the sharp peaks from these sources produce anomalous peaks in the resolution-enhanced spectra. The bands at $2350 \mathrm{~cm}^{-1}$ and $667 \mathrm{~cm}^{-1}$ are due to carbon dioxide, whereas peaks spanning $3900 \mathrm{~cm}^{-1}$ to $3400 \mathrm{~cm}^{-1}$, and $1850 \mathrm{~cm}^{-1}$ to 1350 $\mathrm{cm}^{-1}$ are due to atmospheric water vapor [109]. $\mathrm{N}_{2}$ and $\mathrm{O}_{2}$ molecules do not absorb infrared radiation because of their symmetry. When the sample compartment is opened and closed, the concentrations of $\mathrm{CO}_{2}$ and $\mathrm{H}_{2} \mathrm{O}$ increase and purging the spectrometer with dry nitrogen cannot always reproduce exactly the same $\mathrm{CO}_{2}$ and $\mathrm{H}_{2} \mathrm{O}$ concentrations evident in the background.

\section{4 - DATA ANALYSIS}

The BIO-RAD station was used for data acquisition. Since another software developed by Galactic Industries Corporation called GRAMS/386 ${ }^{\mathrm{TM}}$ was used for the analysis, all the data given by the BIO-RAD software were transfered to another computer. Spectral manipulations, which are discussed in this section, could then be performed.

\subsection{1 - BASELINE CORRECTION}

A sloping or curved baseline can be corrected in order to get a flat baseline, as shown in Figure 3.8. A line segment is drawn between two chosen data points. 


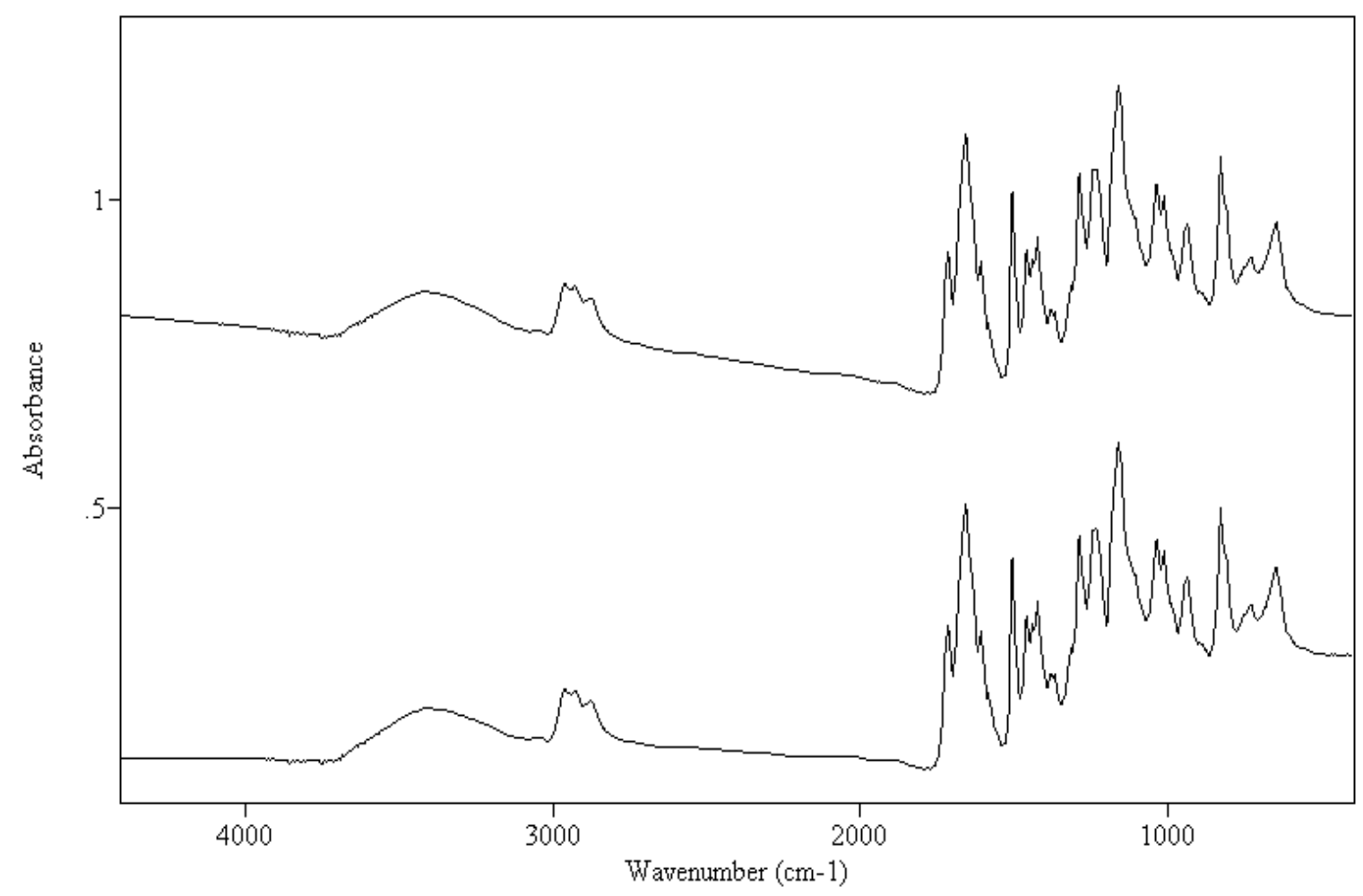

Figure 3.8: Result of baseline correction

(top: baseline slope, bottom: after baseline correction)

\subsection{2 - SMOOTHING}

Smoothing improves the overall appearance of noisy spectra. Noise is reduced and absorbance bands previously unobservable can be apparent. The Savitsky-Golay smoothing algorithm fits a polynomial function to the set of data points in a smoothing window containing $\mathrm{n}$ data points. In our case, a 9 point smooth with a polynomial value of 2 was used. These values were assumed to prevent oversmoothing. Examples of a noisy spectrum and the resulting smooth spectrum are presented in Figures 3.9 and 3.10, respectively. 


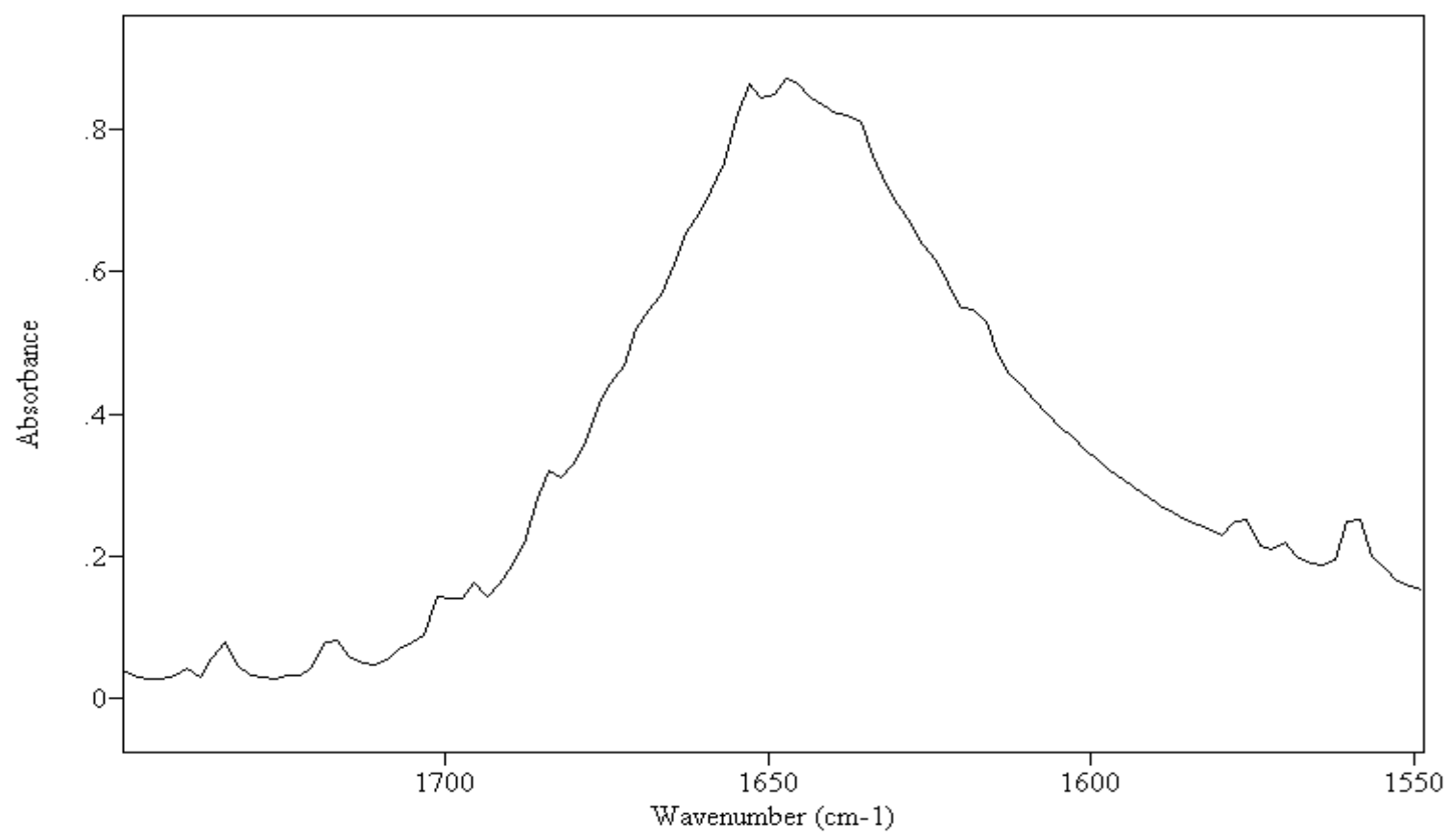

Figure 3.9: A noisy spectrum

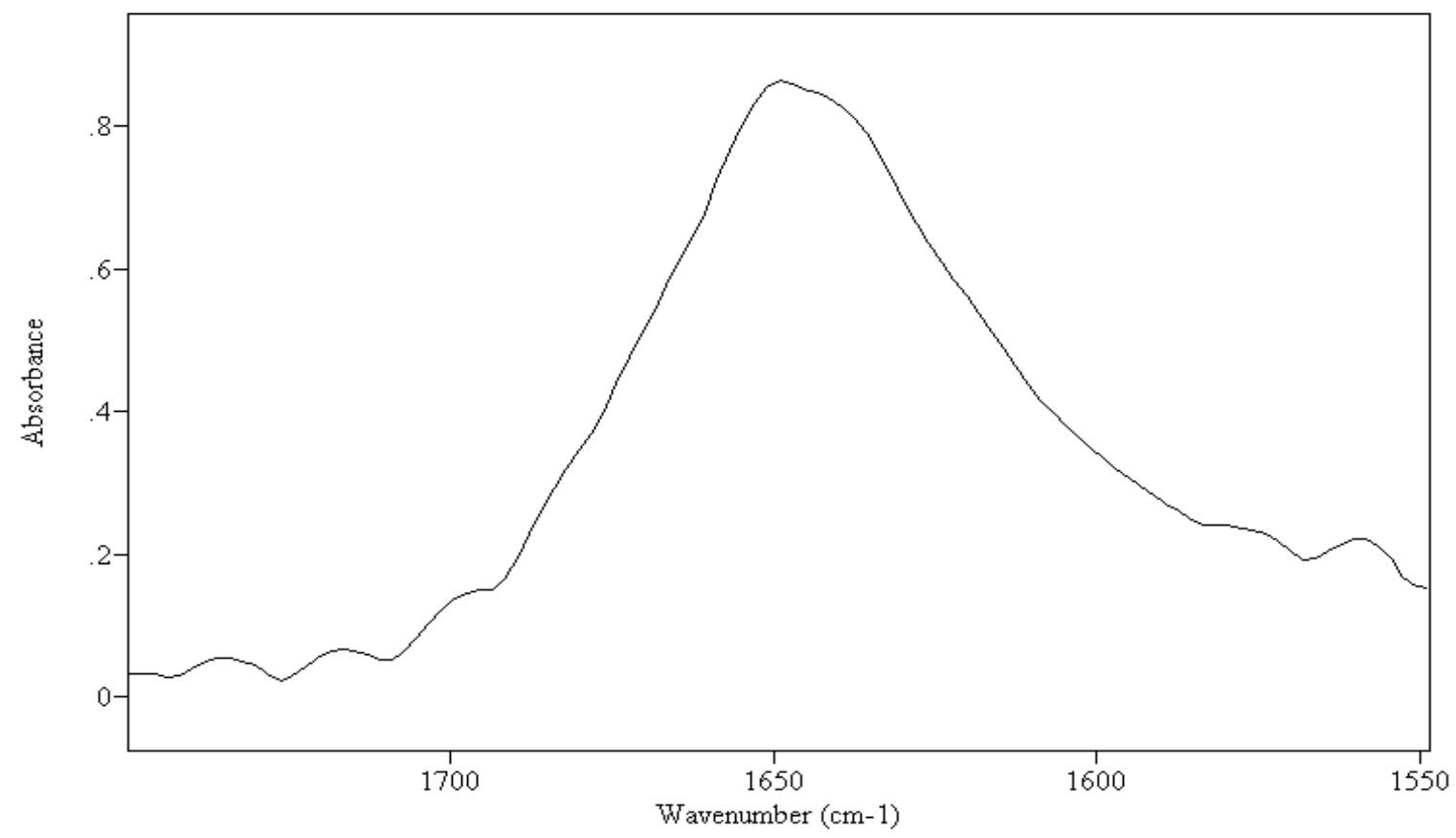

Figure 3.10: The same spectrum after a 9 point Savitsky-Golay smooth 


\subsection{3 - SPECTRAL DERIVATIVE}

The position of the bands can be determined using a second derivative analysis [109]. Since an infrared spectrum is a mathematical function, a second order derivative can be calculated. The second derivative gives a good estimate of the number of bands in the region of interest. In fact, for each band, there are one downward and two upward pointing features in the derivative. The center frequency of the band corresponds exactly to the downward pointing feature of the second derivative. There are several methods to calculate derivatives. In our case, the second derivative was obtained using the Savitsky-Golay method with a degree of polynomial of 2 and a number of convolution points of 5. An example is shown in Figure 3.11.

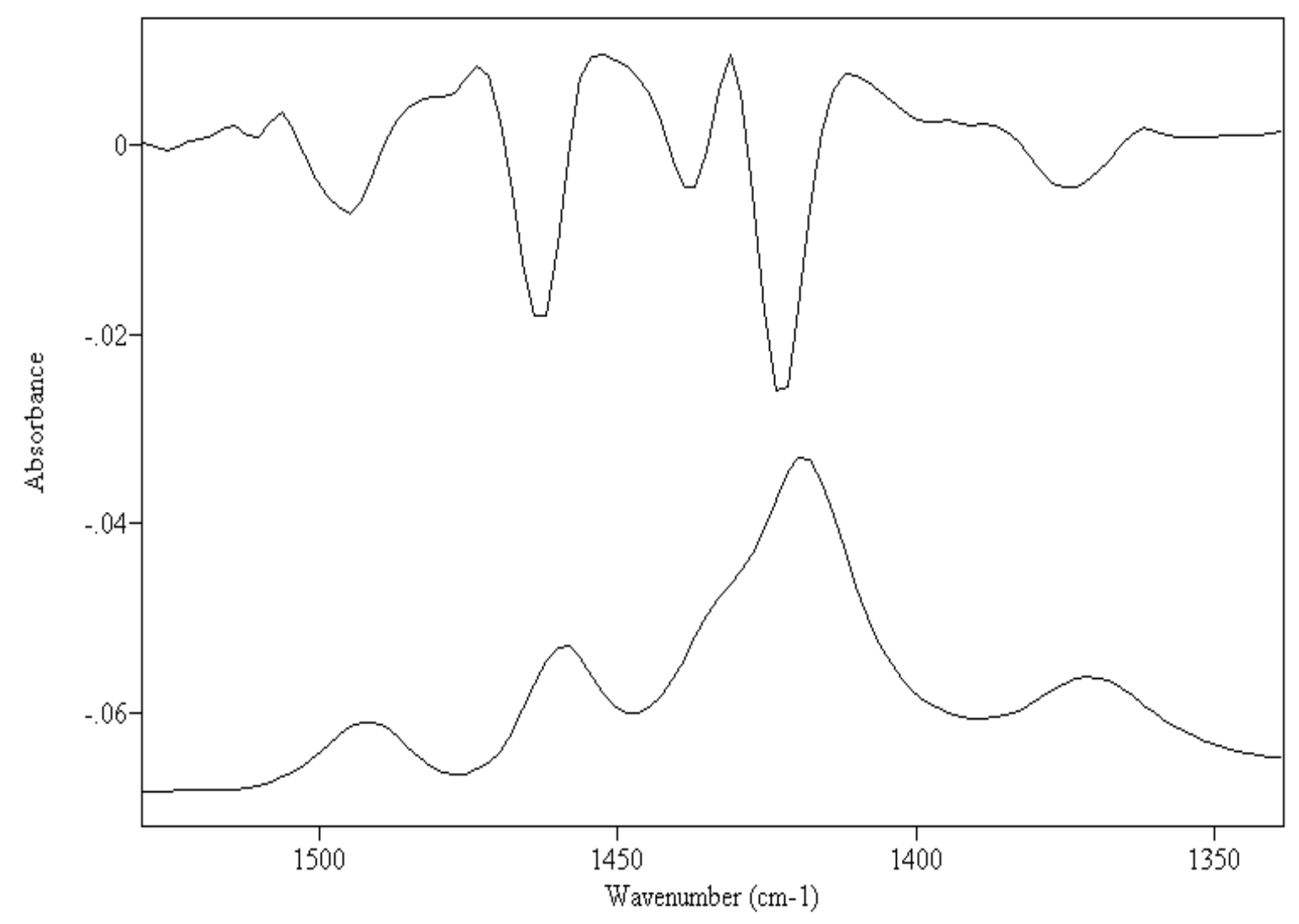

Figure 3.11: Example of a second derivative (bottom: original spectrum, top: the second derivative of this spectrum) 


\subsection{4 - CURVE FITTING}

Curve fitting, which is often called deconvolution by mistake, is a least-squares optimization routine for finding the best collection of individual peaks whose sum closely matches the original spectrum of overlapped bands. It determines the position and intensity of several individual peaks which are overlapped. The program developed for GRAMS uses the Levenberg-Marquardt method. After the spectral region of interest is selected and the baseline is determined, the program then starts by asking the number of peaks and the approximate shape of the peaks (Gaussian, Lorenzian, etc...), their individual frequency positions, and widths at half height. The number of peaks can be determined by looking at the second derivative of the spectrum: the presence of minima indicates the presence of bands. As far as the shapes of the bands are concerned, a sum of Gaussian and Lorenzian functions seems to be the best choice initially. Nevertheless, if the computer finds a better choice, it asks us to change our initial guess. The program then tries different combinations of intensities and widths of these bands, with the best combination being the one whose sum spectrum most closely matches the original spectrum. The result is considered as correct when the fit converges and when $\chi^{2}$, the reduced chi-squared parameter, which represents the difference between the calculated and measured data, is less than 3 .

This curvefitting program has to be used very cautiously since more than one seemingly correct result can be obtained. Different sets of parameters can give equally good results, suggesting that the solution is not unique. One has to carefully select the parameters and the best result, and then determine if that result is reasonable. 


\section{CHAPTER 4}

\section{RESULTS AND DISCUSSION}

The FTIR-ATR technique, detailed in Section 2.3, allows one to observe in situ any spectral change due to the diffusion of a component within the penetration depth. ATR spectroscopy monitors changes in the characteristic absorbance bands of each component of a binary system as a function of time. Diffusion coefficients for each component can then be obtained by fitting the absorbance-time data to a Fickian diffusion model, which is formulated in Subsection 2.4.3.5. The ATR method offers the advantage of measuring diffusion coefficients, as well as characterizing chemical interactions. The ATR technique has been applied in this research to study the molecular diffusion across a poly(vinyl pyrrolidone)/vinyl ester monomer (PVP/VE) interface.

The first section of this chapter focuses on the methodology used in estimating diffusion coefficients by FTIR-ATR spectroscopy and summarizes the results obtained. The following section describes the molecular interactions occuring in the system. The final section provides a discussion, emphasizing the necessity to develop a more appropriate, not-necessarily simply Fickian model for the particular (PVP/VE) system.

\section{1 - DIFFUSION MEASUREMENTS BY FTIR-ATR SPECTROSCOPY}

In this study, a series of ATR diffusion experiments was carried out at three different temperatures, namely $80^{\circ} \mathrm{C}, 90^{\circ} \mathrm{C}$ and $100^{\circ} \mathrm{C}$, in order to assess the temperature dependence of the diffusion coefficient. 
The first subsection in this chapter is devoted to the identification of distinguishable infrared bands for the PVP and the VE. Subsection 4.1.2 describes the evolution of the spectra with time. The following sections provide the methodology used in determining heights of the peaks and diffusion coefficients. The temperature dependence of diffusion coefficients is treated in Subsection 4.1.5, while the activation energy is calculated in Subsection 4.1.6. The concentration profile is determined in Subsection 4.1.7 and finally the interphase thickness is estimated in Subsection 4.1.8.

\subsection{1 - PEAK ASSIGNMENTS}

In order to follow the diffusion of specific components, characteristic frequencies had to be identified. The ATR spectra of the PVP and the VE on a ZnSe crystal at $\theta=45^{\circ}$ were obtained at room temperature. These are shown in Figures 4.1 and 4.2.

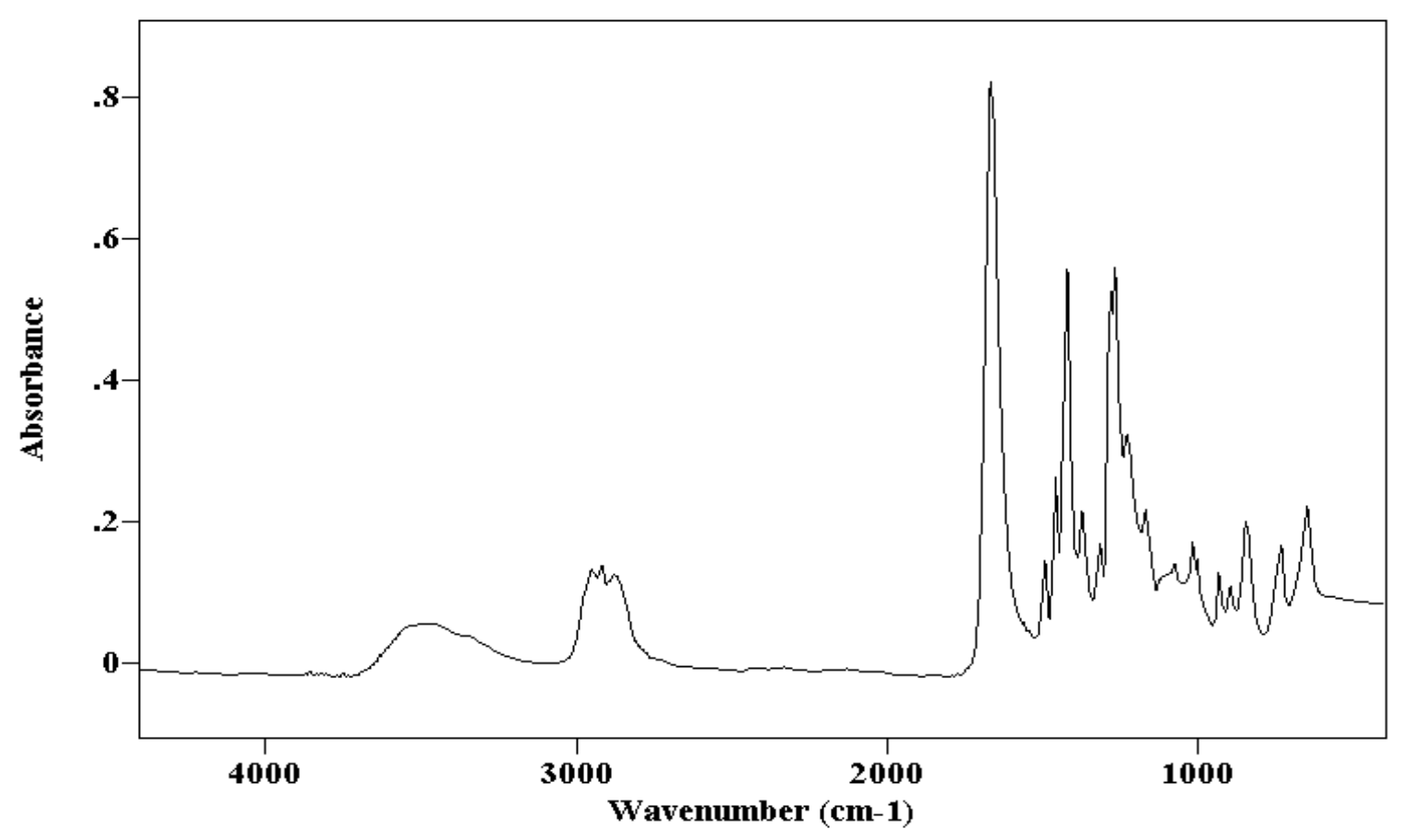

Figure 4.1: Infrared spectrum of the poly(vinyl pyrrolidone) or PVP 


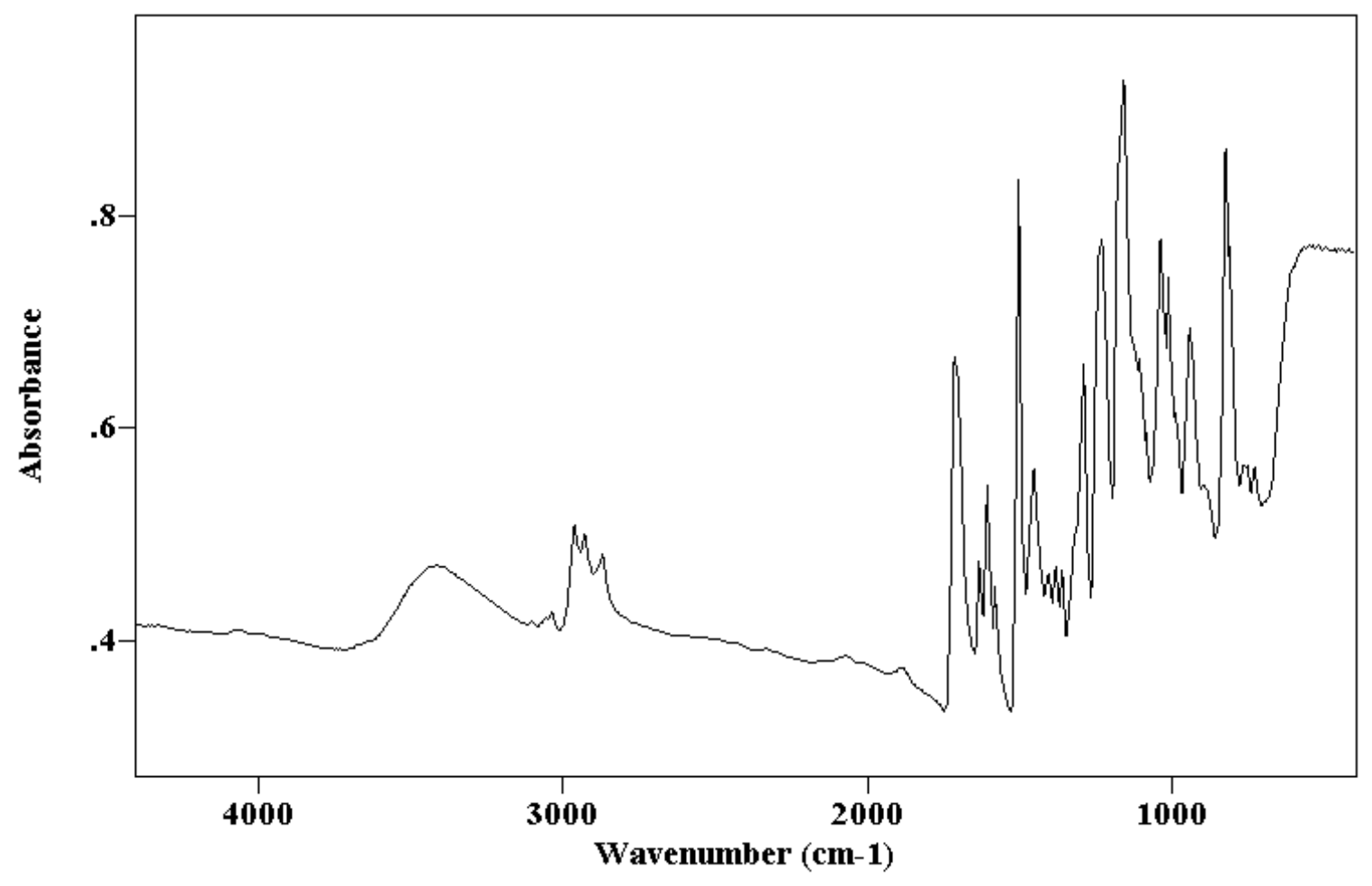

Figure 4.2: Infrared spectrum of the vinyl ester monomer or VE

The probable assignments of the bands in these two spectra were determined for each component. The assignments of the bands of the PVP and those of the VE are given in Table 4.1 and Table 4.2, respectively $[138,139]$. The PVP is hydrophilic and thus some evidence of water can be present in the spectrum. Although all attempts have been made to exclude moisture from the sample, one can not definitely state that there is zero contribution from moisture in the hydroxyl region. In fact, the peak at $3469 \mathrm{~cm}^{-1}$ is assigned to $\mathrm{OH}$ stretching vibrations. 
Table 4.1: Peak assignments of the poly(vinyl pyrrolidone) (PVP)

\begin{tabular}{|r|l|}
\hline position $\left(\mathrm{cm}^{-1}\right)$ & \multicolumn{1}{|c|}{ probable assignment } \\
\hline 3469 & $\mathrm{OH}$ stretch \\
\hline $2948 / 2918 / 2875$ & $\mathrm{CH}$ and $\mathrm{CH}_{2}$ stretch \\
\hline 1664 & contribution from $\mathrm{C}=\mathrm{O}$ and $\mathrm{N}-\mathrm{C}$ stretching vibrations \\
\hline $1492 / 1459 / 1419 / 1371$ & $\mathrm{CH}$ deformation of cyclic $\mathrm{CH}_{2}$ groups \\
\hline $1282 / 1267$ & amide III band $(\mathrm{C}-\mathrm{N}$ stretch $)$ \\
\hline 732 & amide V band or $\mathrm{CH} 2$ rock \\
\hline 648 & amide IV band \\
\hline
\end{tabular}

Table 4.2: Peak assignments of the vinyl ester monomer (VE)

\begin{tabular}{|c|c|}
\hline position $\left(\mathrm{cm}^{-1}\right)$ & probable assignment \\
\hline 3416 & OH stretching vibrations \\
\hline 3036 & $\mathrm{CH}$ stretch of the benzene ring \\
\hline $2962 / 2929 / 2871$ & $\mathrm{CH}, \mathrm{CH}_{2}, \mathrm{CH}_{3}$ stretch \\
\hline 1889 & aromatic ring vibration \\
\hline 1712 & $\mathrm{C}=\mathrm{O}$ stretch \\
\hline 1635 & $\mathrm{C}=\mathrm{C}$ stretch \\
\hline $1606 / 1581 / 1507$ & aromatic ring stretch \\
\hline 1467 & $\mathrm{CH}_{2}, \mathrm{CH}_{3}$ scissors \\
\hline 1454 & aromatic ring stretch \\
\hline 1405 & $\mathrm{CH}_{2}$ around the double band \\
\hline 1382 & $\mathrm{CH}_{2}, \mathrm{CH}_{3}$ scissors \\
\hline 1293 & C-O stretch \\
\hline 1233 & C-O-C stretch \\
\hline 1160 & C-CO-O stretch \\
\hline 1038/1011 & aromatic $\mathrm{C}-\mathrm{H}$ bend \\
\hline 940 & $\mathrm{C}=\mathrm{C}$ stretch \\
\hline 894 & $\mathrm{CH}$ deformations around the double band \\
\hline $767 / 756 / 736$ & aromatic ring stretch \\
\hline 727 & aromatic ring stretch or $\mathrm{CH}_{2}$ rock \\
\hline
\end{tabular}


Once the peak assignments were carried out, distinguishable infrared bands were identified for the two components. Figures 4.3, 4.4 and 4.5 show the comparison between both spectra at different spectral regions.

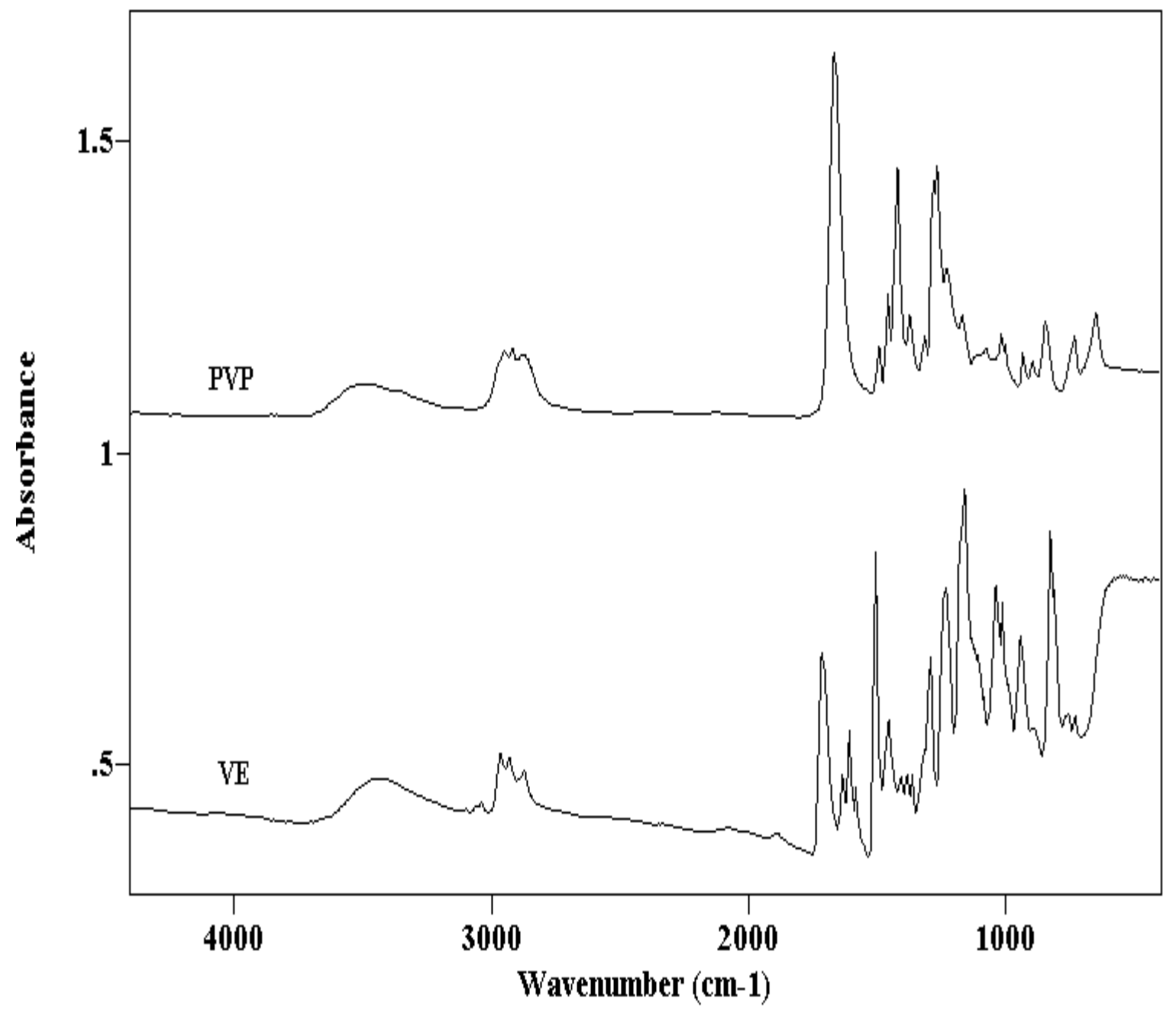

Figure 4.3: Spectra of the PVP and the VE 


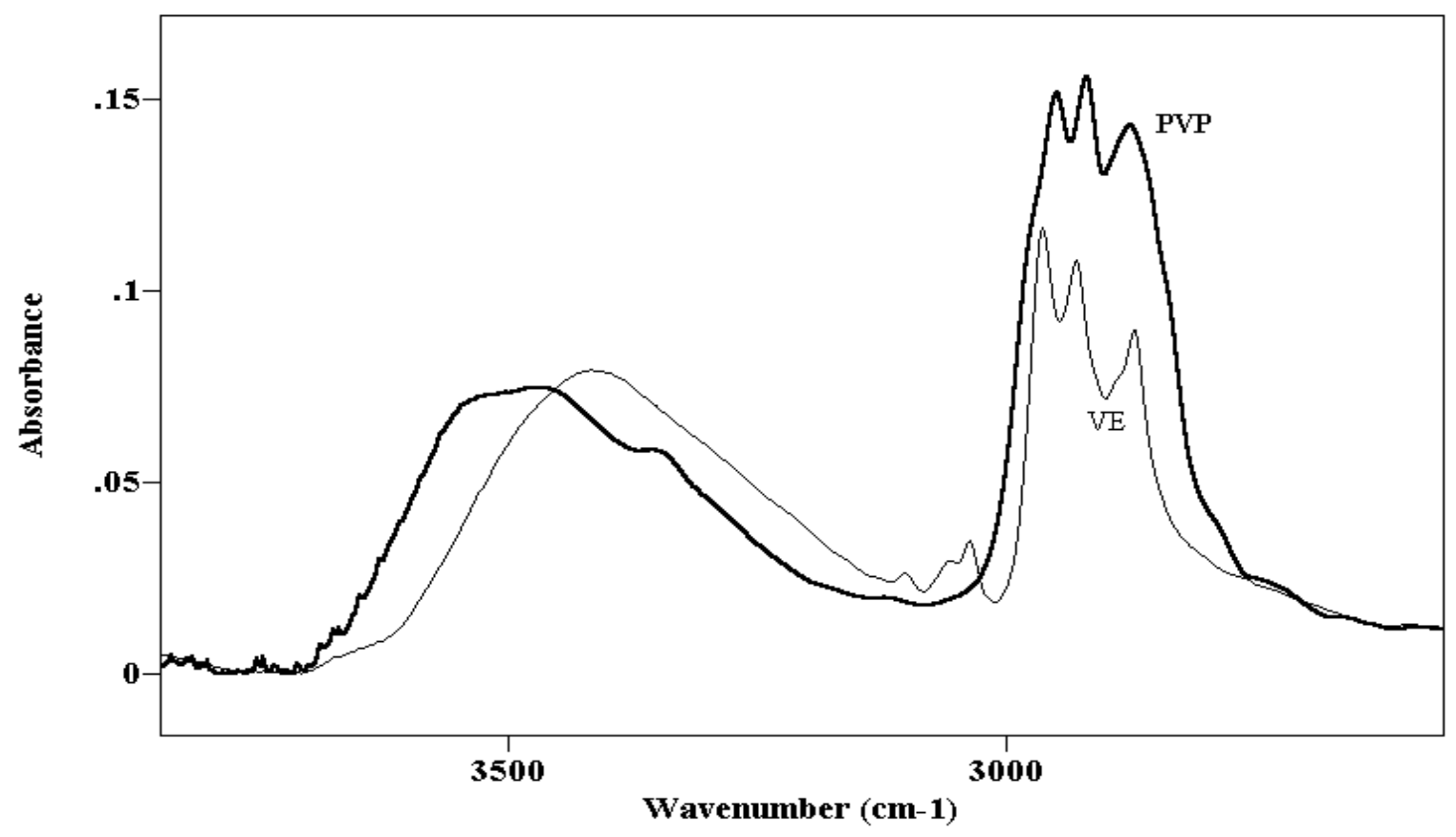

Figure 4.4: Overlapped spectra of the PVP and the VE in the hydroxyl region

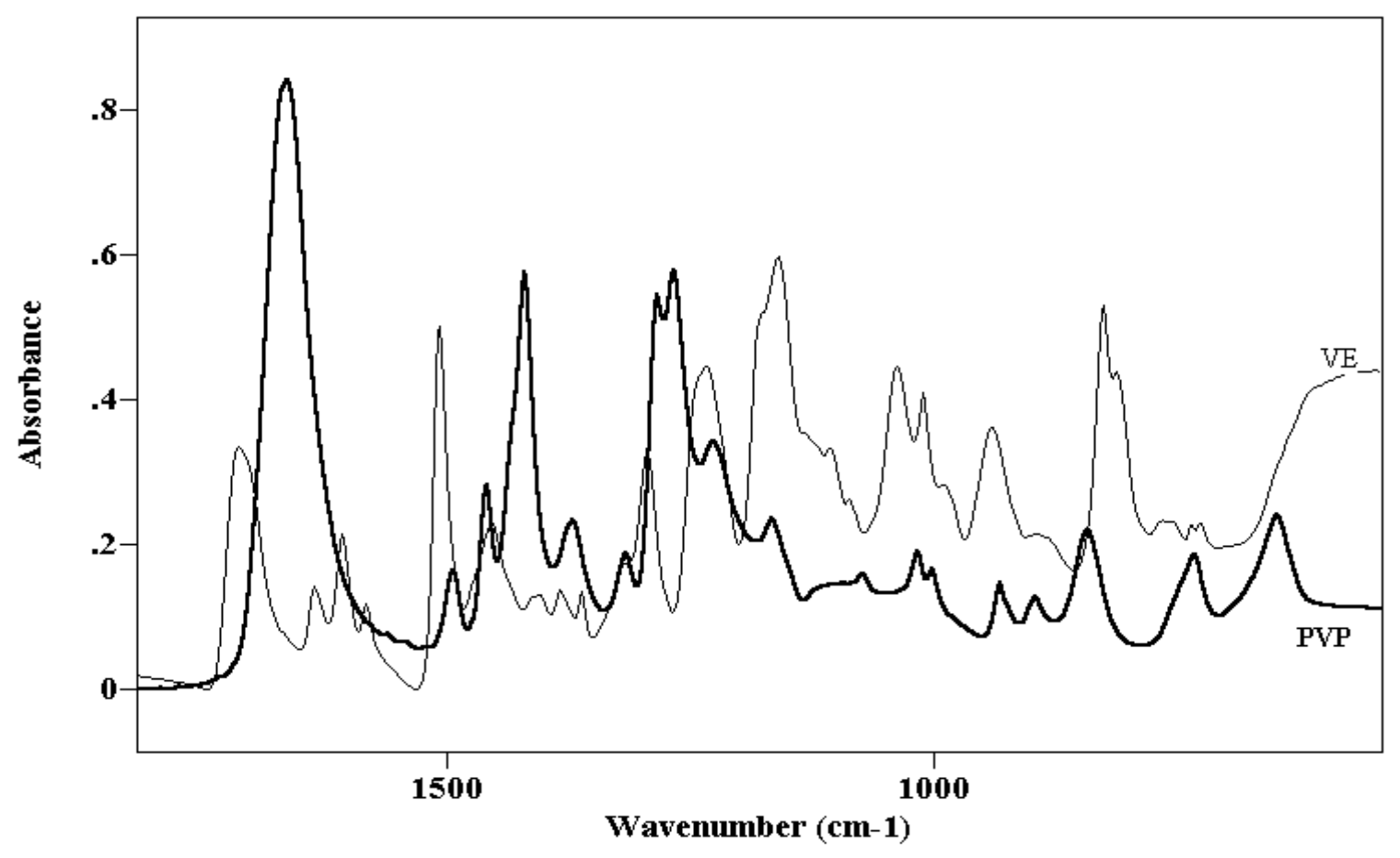

Figure 4.5: Overlapped spectra of the PVP and the VE at low wavenumbers 
The analysis was restricted to typical group vibrations in the characteristic spectral region. The nonoverlapping characteristic bands were selected from one of the principal absorptions of the pure compounds, such as a backbone or skeletal vibrational bands. The bands at $1717 \mathrm{~cm}^{-1}$ and $1507 \mathrm{~cm}^{-1}$ (for the vinyl ester monomer) and the bands at $1664 \mathrm{~cm}^{-1}$ and $1419 \mathrm{~cm}^{-1}$ (for the poly(vinylpyrrolidone)) were used for quantitative analysis of the spectra. The assignments of the peaks of interest are summarized in Table 4.3, while Figure 4.6 shows the overlapped spectra of the PVP and the VE in the region of interest. The peak at $940 \mathrm{~cm}^{-1}$, representative of a $\mathrm{C}=\mathrm{C}$ stretch which disappear with polymerization [140], was not included in the analysis since the bands of the PVP and the VE overlapped at this given wavenumber.

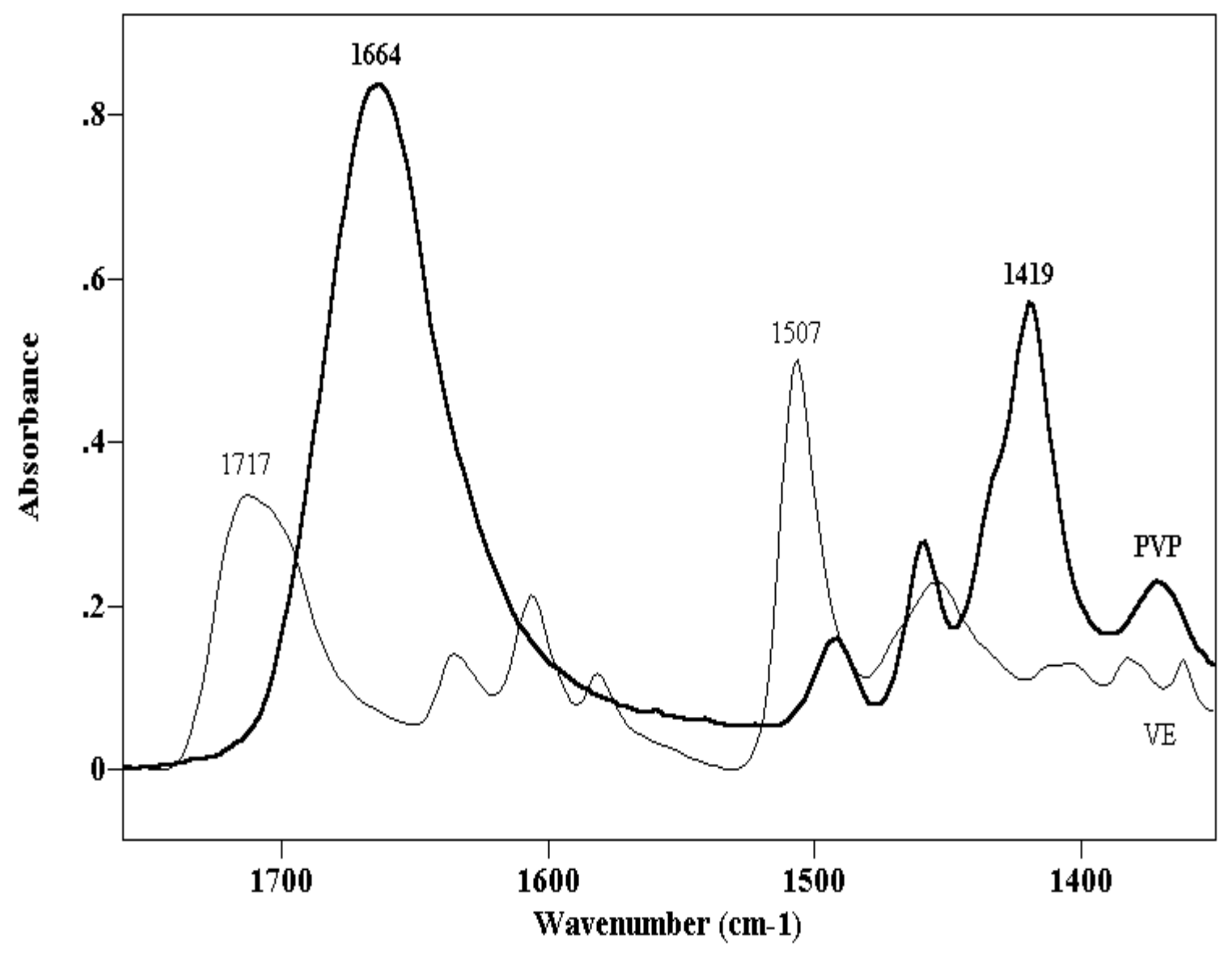

Figure 4.6: Overlapped spectra of the PVP and the VE in the region of interest 
Table 4.3: Assignments of the chosen characteristic bands

\begin{tabular}{|l|l|l|}
\hline compound & position $\left(\mathrm{cm}^{-1}\right)$ & probable assignment \\
\hline VE & 1717 & $\mathrm{C}=\mathrm{O}$ stretch \\
\hline PVP & 1664 & $\mathrm{C}=\mathrm{O}$ and $\mathrm{N}-\mathrm{C}$ stretching vibrations \\
\hline VE & 1507 & aromatic ring stretch \\
\hline PVP & 1419 & $\mathrm{CH}$ deformation of cyclic $\mathrm{CH}_{2}$ groups \\
\hline
\end{tabular}

\subsection{2 - TIME EVOLUTION}

The system consisting of the ATR crystal and the two components was heated to the desired interdiffusion temperature. The diffusion process was then followed by studying the variations of the absorbance of the PVP and the VE infrared bands with time. The temperature was generally controlled to within $2^{\circ} \mathrm{C}$. Spectra were collected every 50 s at the beginning of the experiment (the minimum time necessary between 2 spectra) and then at irregular intervals until the system reached equilibrium. Initial spectra contained some noise as a result of water vapor and carbon dioxide. These spectra were not included in the quantitative analysis.

The changes appeared first at lower wavenumbers of the spectrum. As already mentioned in Section 2.3 and illustrated in Figure 2.5, the light penetrates further into the sample when the wavenumber is lower, and as a result the bands corresponding to the intrusion of the VE are more intense at low wavenumbers than at high wavenumbers. The penetration depths of the chosen characteristics bands, calculated from (2.3.c), are given in Table 4.4.

Figure 4.7 shows the evolution of the spectra with time when $\mathrm{T}=100^{\circ} \mathrm{C}$. As interdiffusion proceeded, the bands at $1507 \mathrm{~cm}^{-1}$ and $1717 \mathrm{~cm}^{-1}$ increased with time, whereas the bands at $1422 \mathrm{~cm}^{-1}$ and $1664 \mathrm{~cm}^{-1}$ decreased with time. As the VE migrated towards the ATR crystal, the VE became more detectable by the evanescent beam. 
Table 4.4: Penetration depths of the peaks of interest

\begin{tabular}{|r|r|}
\hline peak (in cm & dp $($ in $\mu \mathrm{m})$ \\
\hline 1717 & 1.209 \\
\hline 1664 & 1.248 \\
\hline 1507 & 1.378 \\
\hline 1419 & 1.463 \\
\hline
\end{tabular}

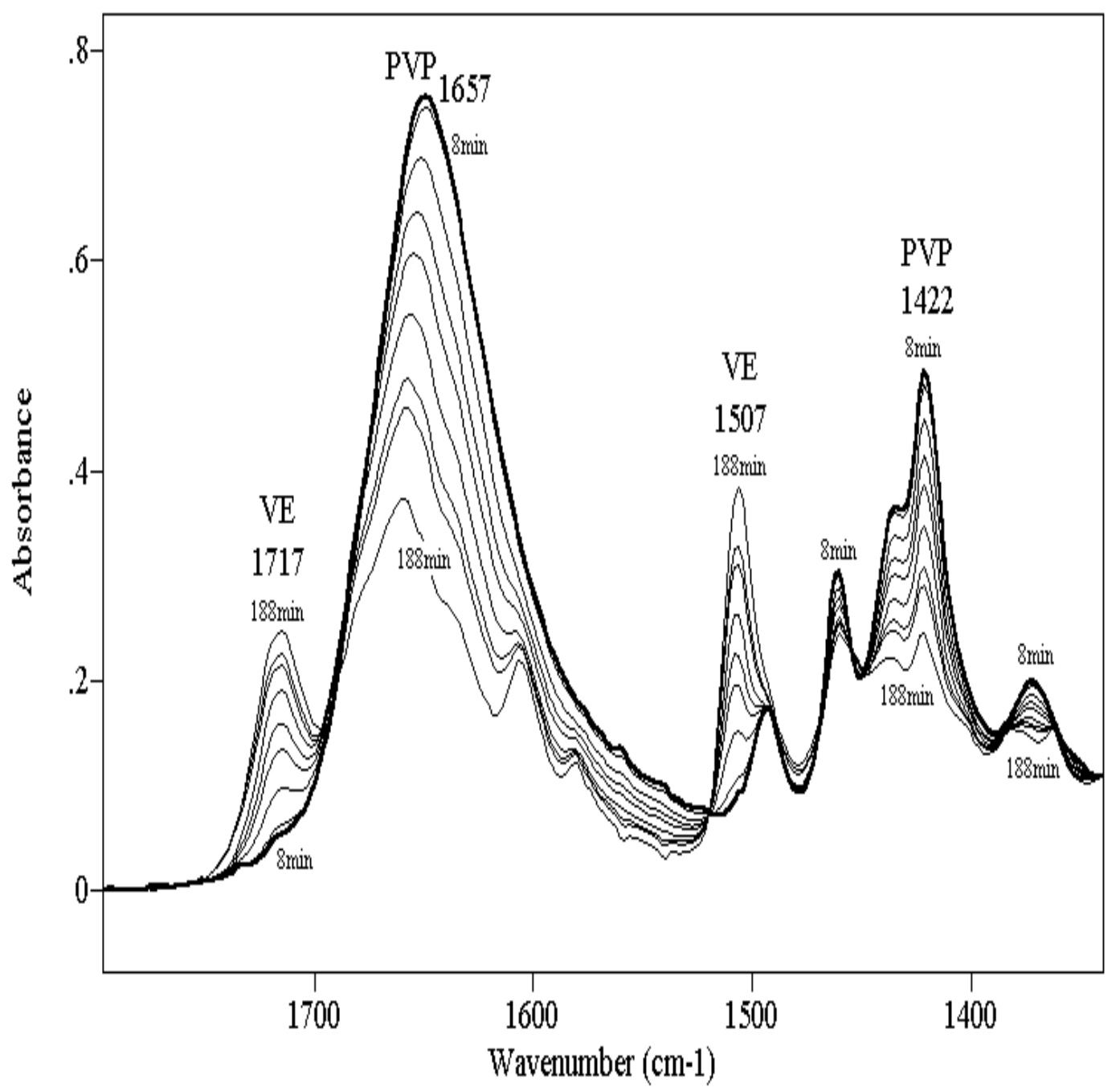

Figure 4.7: Time evolution spectra at $\mathrm{T}=100^{\circ} \mathrm{C}$ from $1850 \mathrm{~cm}^{-1}$ to $1350 \mathrm{~cm}^{-1}$ The interdiffusion times (in minutes) are: 8, 9, 10, 11, 12, 13.7, 21, 32 and 188 


\subsection{3 - DETERMINATION OF THE HEIGHTS OF THE PEAKS}

The heights of the peaks of interest were measured as a function of time. The heights in absorbance units were evaluated for each spectrum by either a peak or a curvefitting program.

The heights of the peaks at $1717 \mathrm{~cm}^{-1}$ and $1507 \mathrm{~cm}^{-1}$, characteristic of the VE, were determined using the GRAMS curve fitting program. An example of the results obtained for the peak at $1507 \mathrm{~cm}^{-1}$ at $\mathrm{T}=80^{\circ} \mathrm{C}$ is given in Appendix B. To verify the consistency of the results obtained by the program, the fit of a particular spectrum was done three times with three different initial guesses for the positions and widths at half height, and the heights were plotted versus time. All data were fit with a master curve and only those values falling directly or close to the master curve were kept for further analysis. Figure 4.8 shows the height of peak $1507 \mathrm{~cm}^{-1}$ as a function of time at $\mathrm{T}=80^{\circ} \mathrm{C}$.

The heights of the peaks at $1664 \mathrm{~cm}^{-1}$ and $1419 \mathrm{~cm}^{-1}$, characteristic of the PVP, were evaluated with respect to baseline points at $1762 \mathrm{~cm}^{-1}$ and $1477 \mathrm{~cm}^{-1}$, respectively. These reference points did not vary in intensity with time. Figure 4.9 shows the results obtained for the peak at $1419 \mathrm{~cm}^{-1}$ at $\mathrm{T}=80^{\circ} \mathrm{C}$.

The results show that with diffusion, the absorbance of the VE peaks increased with time, as the VE chains migrated into the penetration depth, while the concentration of PVP in the same volume decreased with time.

From the peak height versus the interdiffusion time plots, it is possible to obtain $\mathrm{A}(\mathrm{t}), \mathrm{A}_{\mathrm{o}}$, and $\mathrm{A}_{\infty}$, parameters which are needed in the diffusion model formulated by equation (2.4.s). $\mathrm{A}_{\propto}$ was determined from the asymptotic value of the peak height at infinitely long time. 


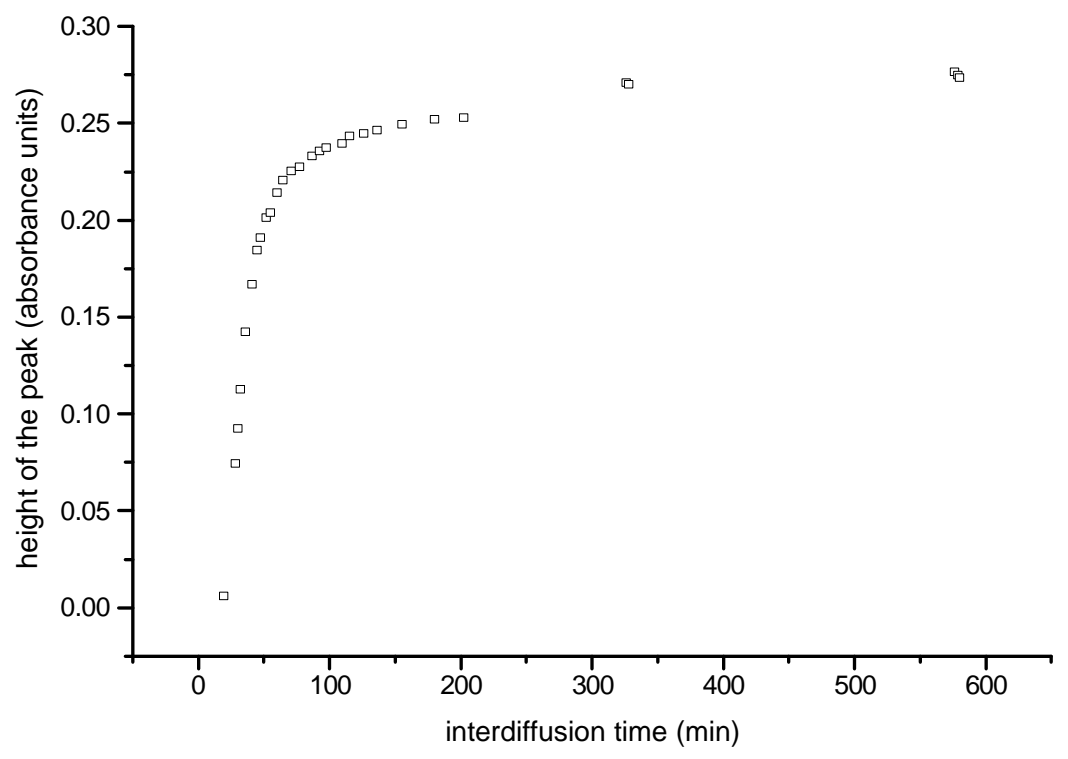

Figure 4.8: Height versus interdiffusion time for the peak at $1507 \mathrm{~cm}^{-1}$ at $\mathrm{T}=80^{\circ} \mathrm{C}$

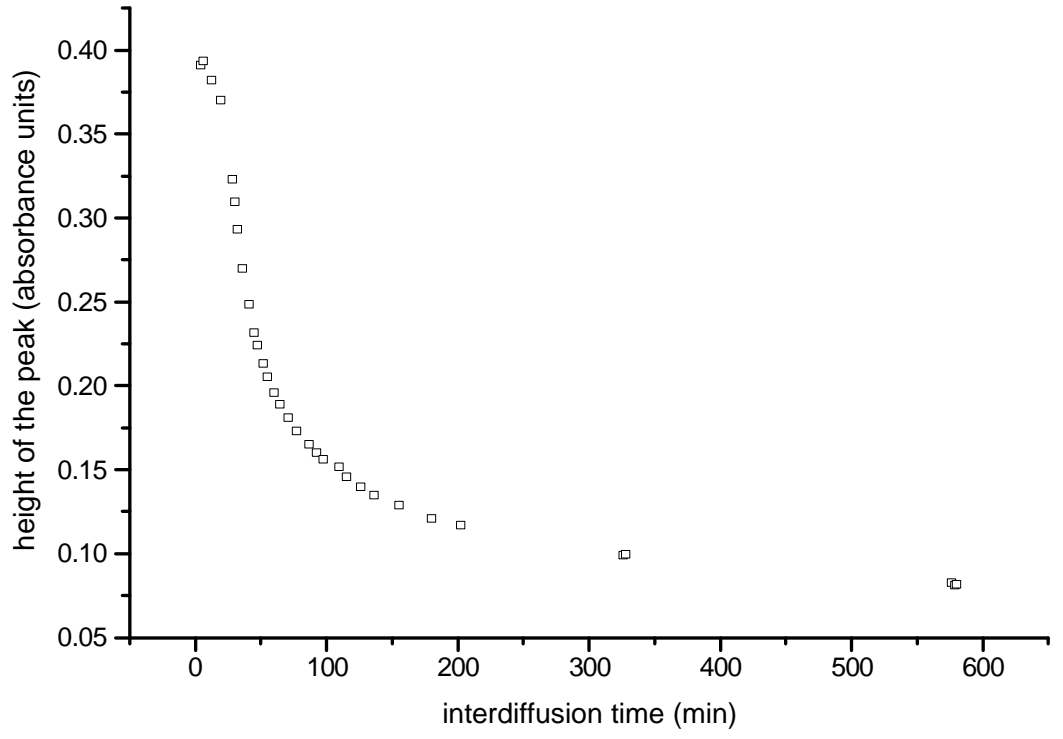

Figure 4.9: Height versus interdiffusion time for the peak at $1419 \mathrm{~cm}^{-1}$ at $\mathrm{T}=80^{\circ} \mathrm{C}$ 
A lapse of time was observed before seeing some changes in the heights of the peaks attributed to the VE. This interval corresponded to the time necessary for the VE to reach the penetration depth. At $\mathrm{T}=80^{\circ} \mathrm{C}$ for instance, the lapse of time was equal to $25 \mathrm{~min}$. Thus 25 min were substracted from all the time values. When studying the disappearance of the PVP, 25 min were also substracted from all the time values for $\mathrm{T}=80^{\circ} \mathrm{C}$, and $\mathrm{A}_{\mathrm{o}}$ was taken as $\mathrm{A}(\mathrm{t})$ at the new time origin. The data obtained before 25 min were not considered. Before the new time origin, the height of the peak of the PVP did not remain exactly constant, as will be discussed in Subsection 4.3.1.

\subsection{4 - DETERMINATION OF THE DIFFUSION COEFFICIENT}

The absorbance-time data could be fit assuming a Fickian diffusion model to obtain the diffusion coefficient of the two components. In order to solve the diffusion model and to get the value of the diffusion coefficient, the experimental data were fit to the equation (2.4.s) by using the simplex optimization algorithm [141] "diff.for" given in Appendix C. In this Fortran program, initial estimations of $\mathrm{D}, \mathrm{A}_{\mathrm{o}}, \mathrm{A}_{\propto}, \mathrm{dp}$, a and $\mathrm{b}$ have to be entered manually. The program then uses these values to curvefit the data, converging to the point for which the error is minimum. The error, defined as:

$$
\text { Error }=\sum_{\mathrm{t}}(\mathrm{A}(\mathrm{t}) \text { exp erimental }-\mathrm{A}(\mathrm{t}) \text { calculated })^{2}
$$

represents the squared difference between the experimental and the calculated absorbance summed over the entire period of time. Optimization of the curve fitting program "diff.for" returns the value of the diffusion coefficient $\mathrm{D}$, as well as $\mathrm{A}_{\propto}$. The program calculates $\mathrm{A}_{\propto}$, which can be compared with $\mathrm{A}_{\propto}$ obtained experimentally. As will be discussed in Subsection 4.3.1, $\mathrm{A}_{\propto}$ obtained experimentally was more accurate than $\mathrm{A}_{\propto}$ calculated from equations (2.4.q) or (2.4.t). Taking the values of $\mathrm{D}$ and $\mathrm{A} \propto$ for which the error was minimum, initial values of $\mathrm{D}$ and $\mathrm{A}_{\propto}$ were adjusted in the computer program and the step of convergence was changed. These steps were repeated until the error was considered as acceptable. 
Plots of the experimental data, along with the curvefit results, were obtained by the use of the Fortran program "curvefit.for" given in Appendix D. Reasonable agreement between the experimental data and the curvefit results were observed. Figures 4.10, 4.11, 4.12, and 4.13 show the plots for the VE and the PVP bands, along with the curvefitting parameters, at $\mathrm{T}=80^{\circ} \mathrm{C}$.

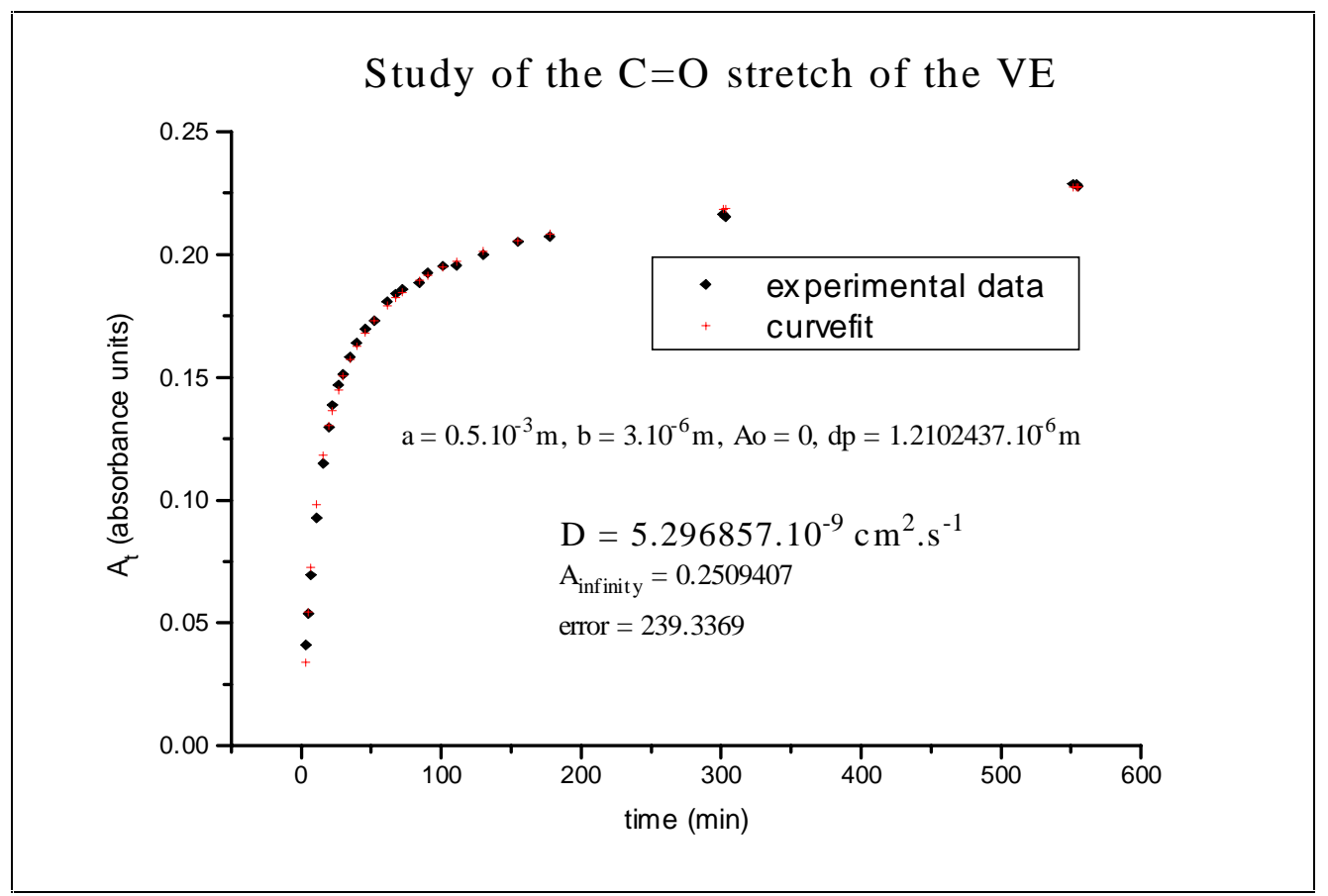

Figure 4.10: Curvefit of the peak at $1716 \mathrm{~cm}^{-1}$ at $\mathrm{T}=80^{\circ} \mathrm{C}$ 


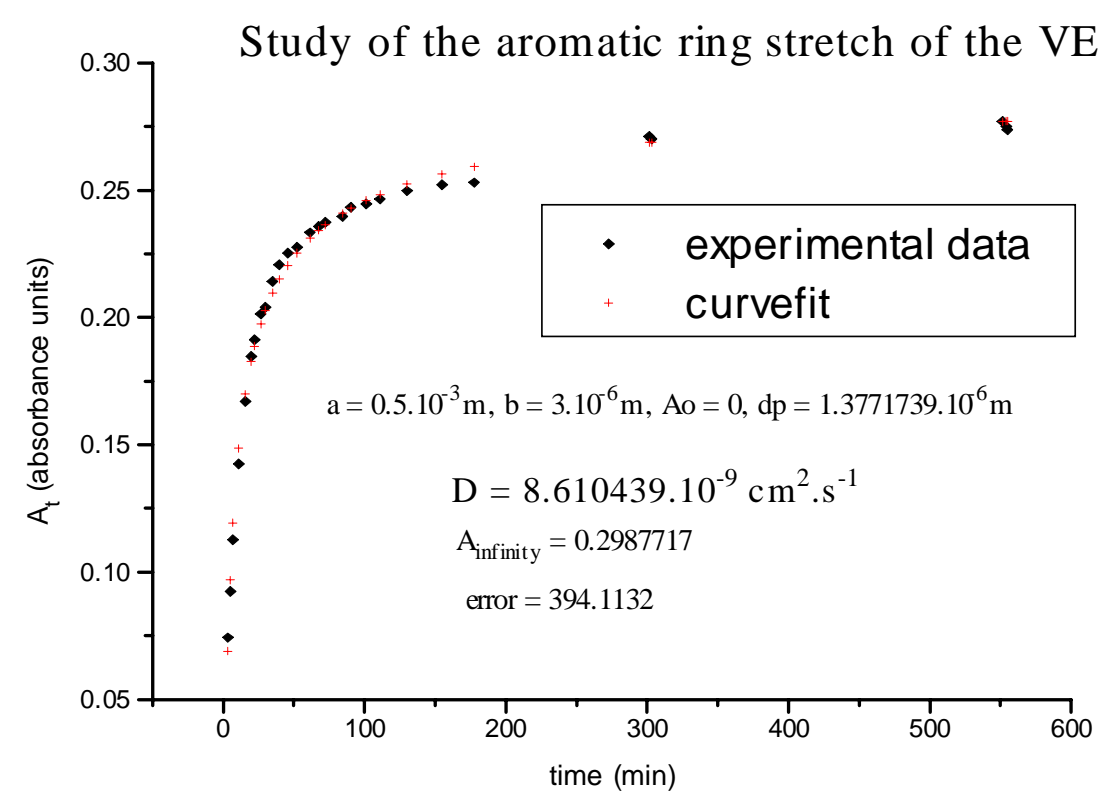

Figure 4.11: Curvefit of the peak at $1508 \mathrm{~cm}^{-1}$ at $\mathrm{T}=80^{\circ} \mathrm{C}$

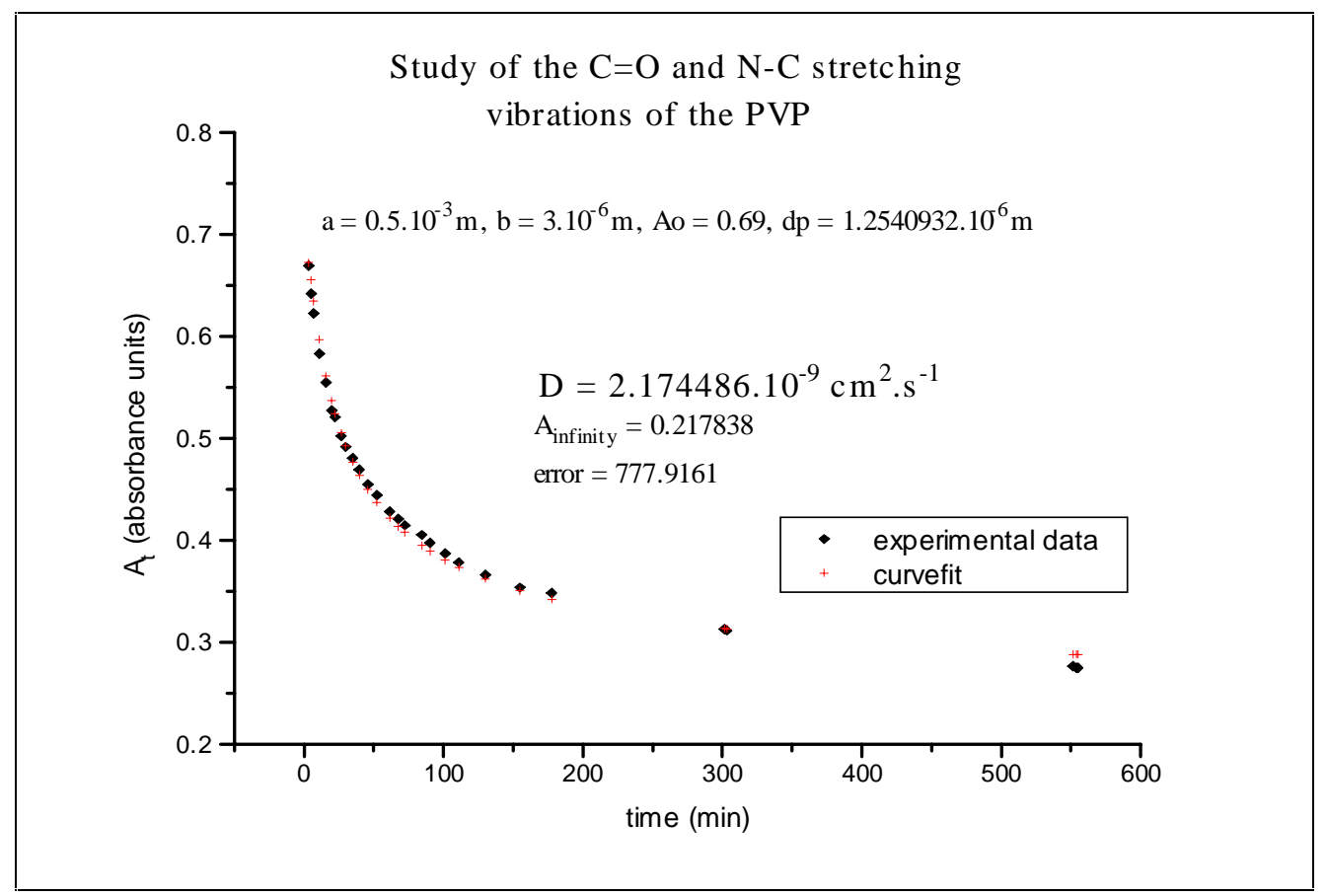

Figure 4.12: Curvefit of the peak at $1656 \mathrm{~cm}^{-1}$ at $\mathrm{T}=80^{\circ} \mathrm{C}$ 


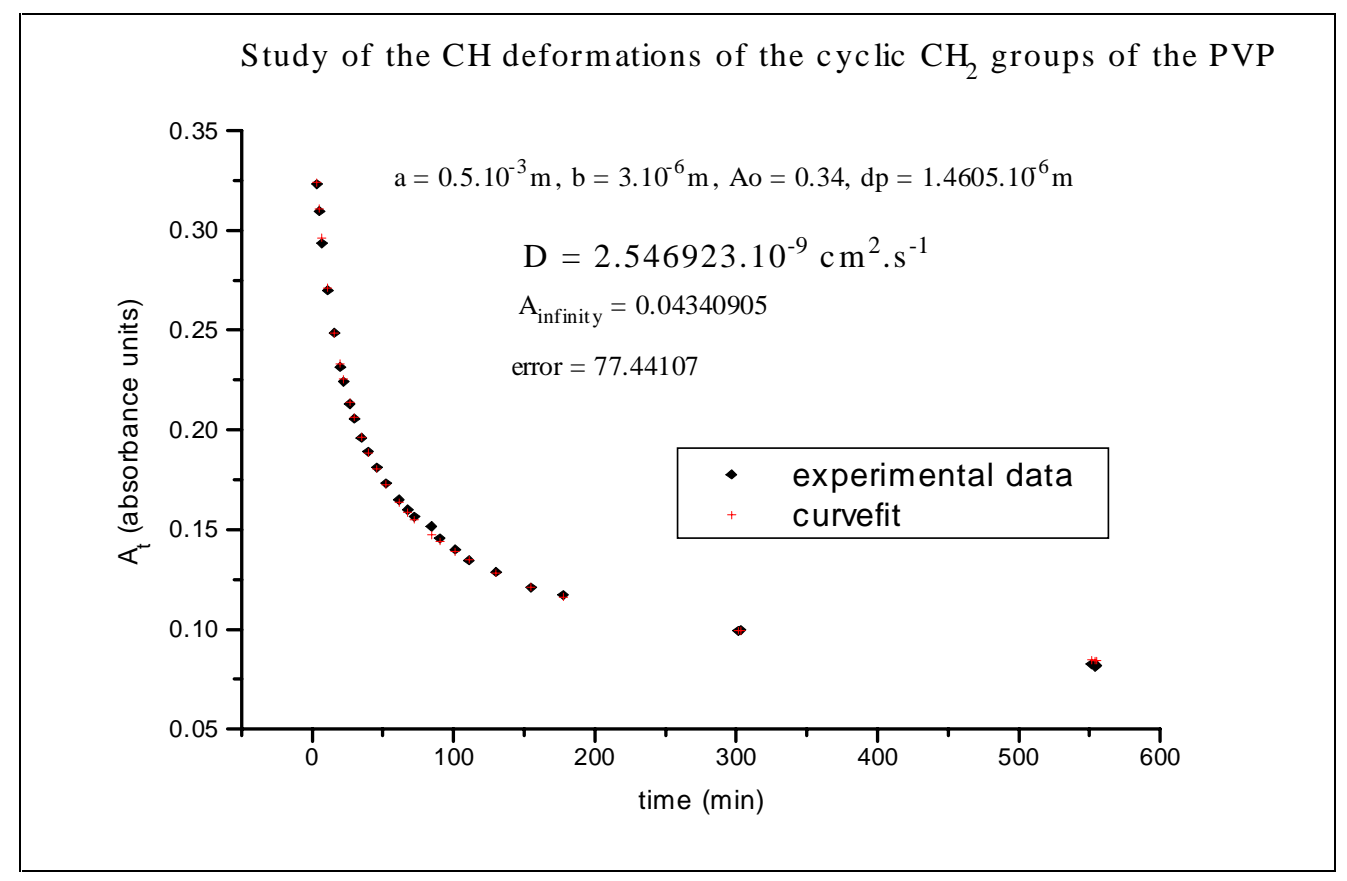

Figure 4.13: Curvefit of the peak at $1422 \mathrm{~cm}^{-1}$ at $\mathrm{T}=80^{\circ} \mathrm{C}$

The diffusion coefficients obtained at $\mathrm{T}=80^{\circ} \mathrm{C}$ are reported in Table 4.5.

Table 4.5: Diffusion coefficients at $\mathrm{T}=80^{\circ} \mathrm{C}$

\begin{tabular}{|l|r|l|}
\hline compound & peak $\left(\mathrm{cm}^{-1}\right)$ & $\mathrm{D}\left(\mathrm{cm}^{2} . \mathrm{s}^{-1}\right)$ \\
\hline VE & 1716 & $8.61 .10^{-9}$ \\
\hline VE & 1508 & $5.3 .10^{-9}$ \\
\hline PVP & 1656 & $2.17 .10^{-9}$ \\
\hline PVP & 1422 & $2.55 .10^{-9}$ \\
\hline
\end{tabular}

The arithmetic mean for the diffusion coefficient of each compound at $\mathrm{T}=80^{\circ} \mathrm{C}$ was calculated. The results are given as follows: $\mathrm{D}_{\mathrm{VE}(80)}=6.95 .10^{-9} \mathrm{~cm}^{2} \cdot \mathrm{s}^{-1}$ and $\mathrm{D}_{\mathrm{PVP}(80)}=$ 2.36.10 $\mathrm{cm}^{2} \cdot \mathrm{s}^{-1}$. $\mathrm{D}_{\mathrm{VE}(80)}$ stands for the diffusion coefficient obtained from the peaks 
characteristic of the $\mathrm{VE}$ at $\mathrm{T}=80^{\circ} \mathrm{C}$, whereas $\mathrm{D}_{\mathrm{PVP}(80)}$ stands for the one obtained from those of the PVP.

$$
\text { Thus: } \quad \frac{\operatorname{DVE}(80)}{\operatorname{DpvP}(80)}=2.95
$$

The fact that the diffusion coefficients of the two components are not the same (which should be the case if one is measuring mutual diffusion coefficients, as is implicit in the Fickian model), suggests that our model is not correct. This topic will be further addressed in the discussion section.

\subsection{5 - EFFECT OF THE TEMPERATURE}

The temperature dependence on the diffusion coefficient of the two components VE and PVP was evaluated by carrying out diffusion experiments at several temperatures and by repeating the analysis of the four characteristic peaks. The position and the shape of those characteristic bands did not change significantly with temperature.

The properties of the interphase depend on the rate of diffusion of the two components, as well as the rate of reaction of the vinyl ester monomer. In order to separate the effects of the VE diffusion and the crosslinking reaction on the infrared spectra, ATR experiments were carried out at temperatures below the normal curing temperature of $150^{\circ} \mathrm{C}$ for the (PVP/VE) system.

A question arises as to whether or not polymerization of the VE could occur during the experiments. In order to check if the VE monomer polymerizes by itself without initiator at high temperature, FTIR transmission spectra of the pure VE were collected. The monomer was sandwiched between two $\mathrm{KBr}$ windows and the spectra were obtained as a function of temperature, ranging from $30^{\circ} \mathrm{C}$ to $113^{\circ} \mathrm{C}$. A spike was observed at the upper temperature for the peak located at $830 \mathrm{~cm}^{-1}$, thus making impossible the continuation of the 
experiments. No changes corresponding to the break of the double bonds were observed before $113^{\circ} \mathrm{C}$.

Differential Scanning Calorimetry (DSC) measurements determined that the polymerization temperature was about $128^{\circ} \mathrm{C}$ at a heating rate of $10^{\circ} \mathrm{C} / \mathrm{min}$. Thus, above $125^{\circ} \mathrm{C}$ in the absence of free radicals, the vinyl groups can still open and form a thermoset polymer.

Respective diffusion coefficients of PVP and VE were obtained at different temperatures, namely 80,90 and $100^{\circ} \mathrm{C}$. The results are summarized in Table 4.6. The diffusion process was strongly influenced by the temperature, which increased the diffusion rates.

The upper temperature of $100^{\circ} \mathrm{C}$ was chosen as a limit. Indeed, the diffusion occurs too rapidly at higher temperatures and cannot be accurately measured with the necessary temporal resolution. One way to study the diffusion at temperatures higher than $100^{\circ} \mathrm{C}$ would be to increase the thickness of the PVP. However, a thicker film of PVP would not have a good contact with the ATR crystal. Furthermore, at higher temperatures, the final compound could crosslink, making removal of the sample from the ATR crystal without damage difficult.

Table 4.6: Diffusion coefficients at different temperatures

\begin{tabular}{|c|c|c|c|c|c|}
\hline & & $\mathrm{T}=80^{\circ} \mathrm{C}$ & $\mathrm{T}=80^{\circ} \mathrm{C}$ & $\mathrm{T}=90^{\circ} \mathrm{C}$ & $\mathrm{T}=100^{\circ} \mathrm{C}$ \\
\hline & & old crystal & new crystal & new crystal & old crystal \\
\hline \multicolumn{2}{|l|}{ time origin } & $\mathrm{t}=60.17 \mathrm{~min}$ & $\mathrm{t}=25 \mathrm{~min}$ & $\mathrm{t}=32.7 \mathrm{~min}$ & $\mathrm{t}=8 \mathrm{~min}$ \\
\hline compound & peak $\left(\mathrm{cm}^{-1}\right)$ & $\mathrm{D}\left(\mathrm{cm}^{2} \cdot \mathrm{s}^{-1}\right)$ & $\mathrm{D}\left(\mathrm{cm}^{2} \cdot \mathrm{s}^{-1}\right)$ & $\mathrm{D}\left(\mathrm{cm}^{2} \cdot \mathrm{s}^{-1}\right)$ & $\mathrm{D}\left(\mathrm{cm}^{2} \cdot \mathrm{s}^{-1}\right)$ \\
\hline $\mathrm{VE}$ & 1717 & $3.07 .10^{-9}$ & $8.61 .10^{-9}$ & $9.25 .10^{-9}$ & $2.15 .10^{-8}$ \\
\hline VE & 1507 & $4.05 .10^{-9}$ & $5.3 .10^{-9}$ & $1.40 .10^{-8}$ & $2.19 .10^{-8}$ \\
\hline PVP & 1657 & $1.63 .10^{-9}$ & $2.17 .10^{-9}$ & $4.89 .10^{-9}$ & $1.39 .10^{-8}$ \\
\hline PVP & 1422 & $1.69 .10^{-9}$ & $2.55 .10^{-9}$ & $4.98 .10^{-9}$ & $1.51 .10^{-8}$ \\
\hline
\end{tabular}


Diffusion coefficients were determined by monitoring two distinguishable infrared bands for each of the PVP and the VE components. The diffusion coefficients obtained from those four specific bands were named $\mathrm{D}_{(\mathrm{PVP})}$ and $\mathrm{D}_{(\mathrm{VE})}$, respectively. Ideally, the value of the diffusion coefficient should be the same for a given component at a fixed temperature, whatever the characteristic band. Ratios of the two characteristic bands of a given compound are presented in Table 4.7.

Table 4.7: Ratio of diffusion coefficients based on different spectral bands

\begin{tabular}{|l|l|r|r|}
\hline & & $\mathrm{D}_{(\mathrm{PVP} / 1422 \mathrm{~cm}-1)} / \mathrm{D}_{(\mathrm{PVP} / 1657 \mathrm{~cm}-1)}$ & $\mathrm{D}_{(\mathrm{VE} / 1507 \mathrm{~cm}-1)} / \mathrm{D}_{(\mathrm{VE} / 1717 \mathrm{~cm}-1)}$ \\
\hline $\mathrm{T}=80^{\circ} \mathrm{C}$ & old crystal & 1.037 & 1.319 \\
$\mathrm{~T}=80^{\circ} \mathrm{C}$ & new crystal & 1.175 & 0.616 \\
\hline $\mathrm{T}=90^{\circ} \mathrm{C}$ & new crystal & 1.018 & 1.513 \\
\hline $\mathrm{T}=100^{\circ} \mathrm{C}$ & old crystal & 1.086 & 1.019 \\
\hline
\end{tabular}

The results obtained for $\mathrm{D}_{(\mathrm{PVP})}$ seem more accurate than the ones obtained for $\mathrm{D}_{(\mathrm{VE})}$. Indeed, the ratio of the two values of $\mathrm{D}_{(\mathrm{PVP})}$ obtained for a given temperature is closer to 1 , as shown in Table 4.7. The difference observed between the two values of $\mathrm{D}_{(\mathrm{VE})}$ can be due to experimental artifacts, such as the curvefitting technique. The discrepancies in the ratio may also reflect the selective diffusion of particular structural elements or groups.

When using FTIR-ATR spectroscopy to measure mutual diffusion coefficients, at least two peaks for each component should be selected, and therefore at least four values of diffusion coefficients for a given temperature should be obtained. Typical diffusion studies cited in the literature show that only one peak is generally analyzed [20, 22, 23], and this peak is the one corresponding to the uptake of a component, i. e. the vinyl ester monomer in our case. However, the results obtained for the polymer directly in contact with the ATR crystal seem more accurate, based on the quality and consistency of the fit. 
An arithmetic mean of the diffusion coefficients for each component was calculated for each temperature. The results are shown in Table 4.8. The diffusion coefficient of the peak at $1507 \mathrm{~cm}^{-1}$ at $90^{\circ} \mathrm{C}$ was not considered in the calculations since the plot of the height of the peak as a function of time was not consistent with the rest of the data. Table 4.9 summarizes the ratio of the diffusion coefficients of the two components

Table 4.8: Average diffusion coefficients at different temperatures

\begin{tabular}{|l|l|l|l|l|}
\hline & $\mathrm{T}=80^{\circ} \mathrm{C}$ & $\mathrm{T}=80^{\circ} \mathrm{C}$ & $\mathrm{T}=90^{\circ} \mathrm{C}$ & $\mathrm{T}=100^{\circ} \mathrm{C}$ \\
\hline $\mathrm{D}\left(\mathrm{cm}^{2} \cdot \mathrm{s}^{-1}\right)$ & old crystal & new crystal & new crystal & old crystal \\
\hline $\mathrm{D}_{\text {(VE) }}$ & $3.56 .10^{-9}$ & $6.95 .10^{-9}$ & $9.25 .10^{-9}$ & $2.17 .10^{-8}$ \\
\hline $\mathrm{D}_{(\mathrm{PVP})}$ & $1.66 .10^{-9}$ & $2.36 .10^{-9}$ & $4.93 .10^{-9}$ & $1.45 .10^{-8}$ \\
\hline
\end{tabular}

Table 4.9: Ratio of $\left(\mathrm{D}_{(\mathrm{VE})} / \mathrm{D}_{(\mathrm{PVP})}\right)$

\begin{tabular}{|l|l|r|}
\hline & & $\mathrm{D}_{(\mathrm{VE})} / \mathrm{D}_{(\mathrm{PVP})}$ \\
\hline $\mathrm{T}=80^{\circ} \mathrm{C}$ & old crystal & 2.14 \\
\hline $\mathrm{T}=80^{\circ} \mathrm{C}$ & new crystal & 2.95 \\
\hline $\mathrm{T}=90^{\circ} \mathrm{C}$ & new crystal & 1.88 \\
\hline $\mathrm{T}=100^{\circ} \mathrm{C}$ & old crystal & 1.5 \\
\hline
\end{tabular}

Table 4.8 indicates that the two sets of results obtained at $\mathrm{T}=80^{\circ} \mathrm{C}$ are not reproducible. One set of results was obtained with an old IRE crystal, whereas a new crystal was used for the other set of results. The lapse of time observed before seeing some changes in the spectrum was 2.4 times less in the case of the new crystal, as reported in Table 4.6, due to different thicknesses of the PVP intermediate layer. In addition, the difference between the values of the diffusion coefficients may be attributed to the difficulty of having a reproducible constant and uniform pressure on the sample. 
The fact that the ratio and $\left(\mathrm{D}_{(\mathrm{VE})} / \mathrm{D}_{(\mathrm{PVP})}\right)$ is much greater than 1 , at a given temperature (see Table 4.9), cannot be entirely due to experimental errors. As a reminder, $\mathrm{D}_{(\mathrm{PVP})}$ and $\mathrm{D}_{\text {(VE) }}$ represent mutual diffusion coefficients of the PVP and the VE, respectively. Diffusion in the (PVP/VE) system takes place in both directions, the VE into the PVP, and vice versa. Hence $\mathrm{D}_{(\mathrm{PVP})}$ and $\mathrm{D}_{(\mathrm{VE})}$ should be identical $[29,30,142,31]$. As already mentioned in Subsection 2.1.1, three distincts diffuson coefficients can be measured for a binary system: the mutual diffusion coefficient, and the two self-diffusion coefficients. The self-diffusion coefficient of the PVP is expected to be many orders of magnitude lower than that of the VE, due to molecular weight differences [31]. However, the mutual diffusion coefficient of the PVP in the VE, and that of the VE in the PVP, should have the same value. Chih-ch'iian [142] demonstrated the fact that there is only one coefficient of molecular diffusion for both components for a binary system. The active diffusion coefficient of one component in one direction is compensated by the passive diffusion of the other component in the opposite direction. Since the two components are transferred simultaneously, there is a single interdiffusion coefficient. That is the reason why only one value of the mutual diffusion coefficient can be found in the literature. At $80^{\circ} \mathrm{C}, \mathrm{D}_{(\mathrm{VE})}$ has been found to be almost three times greater than $\mathrm{D}_{(\mathrm{PVP})}$ (see Table 4.9). Even at $100^{\circ} \mathrm{C}$, temperature for which the results seem quite accurate (see Table 4.7$), \mathrm{D}_{(\mathrm{VE})}$ is still 1.5 times greater than $\mathrm{D}_{(\mathrm{PVP})}$ (see Table 4.9).

Nevertheless, the order of magnitude of the diffusion coefficients is consistent with the range found in the literature for diffusion in polymers. The polymer handbook [143] gives a value of $9.6 .10^{-7} \mathrm{~cm}^{2} . \mathrm{s}^{-1}$ for the diffusion coefficient of water in PVP at $\mathrm{T}=25^{\circ} \mathrm{C}$. Dr. Hideko Oyama [144] estimated the diffusion coefficient for the (PVP/VE) system to be on the order of $10^{-7} \mathrm{~cm}^{2} \cdot \mathrm{s}^{-1}$ at $\mathrm{T}=150^{\circ} \mathrm{C}$.

\subsection{6 - DETERMINATION OF THE ACTIVATION ENERGY}

The diffusion results obtained at different temperatures were fit with an Arrhenius type equation $[28,134]$ : 


$$
D=D_{0} \cdot \exp \left(\frac{-E_{a}}{R T}\right)
$$

where $E_{a}$ is the activation energy of diffusion

$\mathrm{D}_{\mathrm{o}}$ is the preexponential factor

The slope of $(\operatorname{lnD})$ versus $(1 / \mathrm{RT})$ gives us $\left(-\mathrm{E}_{\mathrm{a}}\right)$ and the intercept $\left(\ln \mathrm{D}_{\mathrm{o}}\right)$. The fit of the Arrhenius equation is demonstrated in Figures 4.14 and 4.15 for the PVP and the VE, respectively. The dependence of diffusion coefficients on temperature follows the Arrhenius equation quite well, especially for the PVP. The results obtained for the activation energies and the preexponential factors are summarized in Table 4.10. A fit of the data with the Arrhenius equation gave a value of $79 \mathrm{~kJ} / \mathrm{mol}$ for the activation energy of diffusion of VE, and $108 \mathrm{~kJ} / \mathrm{mol}$ for that of the PVP. The difference in the activation energies of both components is thus equal to $28.67 \mathrm{~kJ} / \mathrm{mol}$.

Table 4.10: Activation energies and preexponential factors

\begin{tabular}{|l|r|r|}
\hline & \multicolumn{1}{|l|}{ VE } & PVP \\
\hline $\mathrm{D}_{\mathrm{o}}\left(\mathrm{cm}^{2} . \mathrm{s}^{-1}\right)$ & 2545.67 & $177.33 .10^{5}$ \\
\hline $\mathrm{E}_{\mathrm{a}}(\mathrm{kJ} / \mathrm{mol})$ & 79.23 & 107.9 \\
\hline
\end{tabular}

The value of the activation energy is consistent with the literature. Of course, a given value cannot be strictly compared with the (PVP/VE) system. Nevertheless, the literature can provide some ideas on the range usually observed for the activation energy of diffusion in polymers. The activation energy of various solvents in PMMA ranged from 19 to 35 $\mathrm{kcal} / \mathrm{mol}$ [145], and that of bis(2-ethylhexyl)phthalate in PVC was measured as $29 \mathrm{kcal} / \mathrm{mol}$ [146]. One example of activation energy for the molecular transport of middle-size molecules on the order of $337 \mathrm{~g} / \mathrm{mol}$ was for instance $100 \mathrm{~kJ} / \mathrm{mol}$, determined for the diffusion of erucamide in isotactic polypropylene [147]. As far as the diffusion of polymers within polymers is concerned, an activation energy of $11.7 \mathrm{kcal} / \mathrm{mol}$ was found for the (PVC/poly(c-caprolactone))/system [148]. 


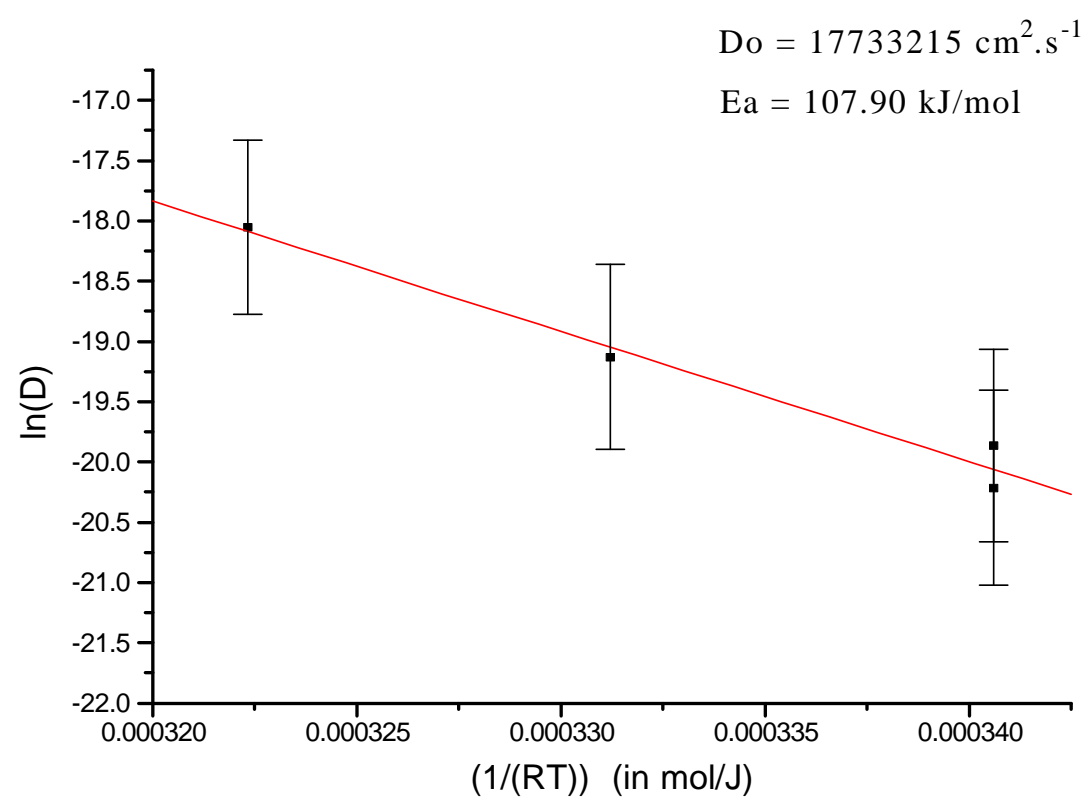

Figure 4.14: Use of the Arrhenius equation for the PVP

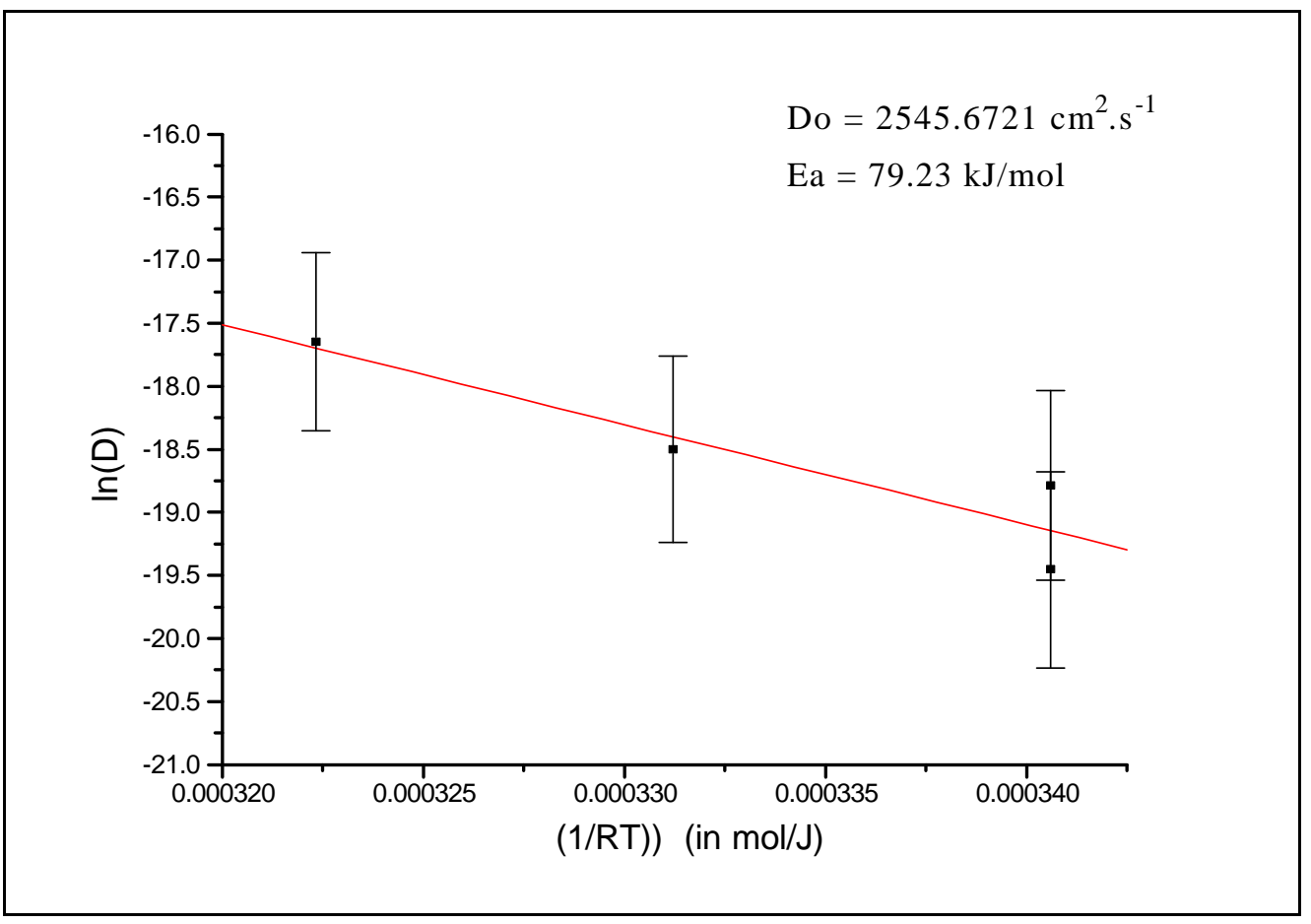

Figure 4.15: Use of the Arrhenius equation for the VE 


\subsection{7 - DETERMINATION OF THE CONCENTRATION CHANGES WITH TIME}

Once the value of the diffusion coefficient assuming a Fickian model is known, it is possible to obtain the concentration profile of the interphase by the equation (2.4.o). In our case, concentration profiles were not calculated, because the actual diffusion coefficients varied with concentration and distance. This effect is discussed in Subsection 4.3.2. However, one can estimate the change in concentration with time at a given penetration depth.

The arithmetic mean of the penetration depths of the two PVP characteristic bands was calculated. The change in concentration at a given point, the point located at the limit of the mean penetration depth, could thus be followed. The value of $\mathrm{D}_{(\mathrm{PVP})}$ was input in equation (2.4.o). The Fortran program "conc2.for" given in Appendix E was used to calculate the concentration profile. The concentration profile obtained at $\mathrm{T}=100^{\circ} \mathrm{C}$ is shown in Figure 4.16. As soon as the VE reached the mean penetration depth (at $\mathrm{t}=8 \mathrm{~min}$ at $\mathrm{T}=100^{\circ} \mathrm{C}$ ), the concentration of the PVP at the mean penetration depth decreased significantly. 


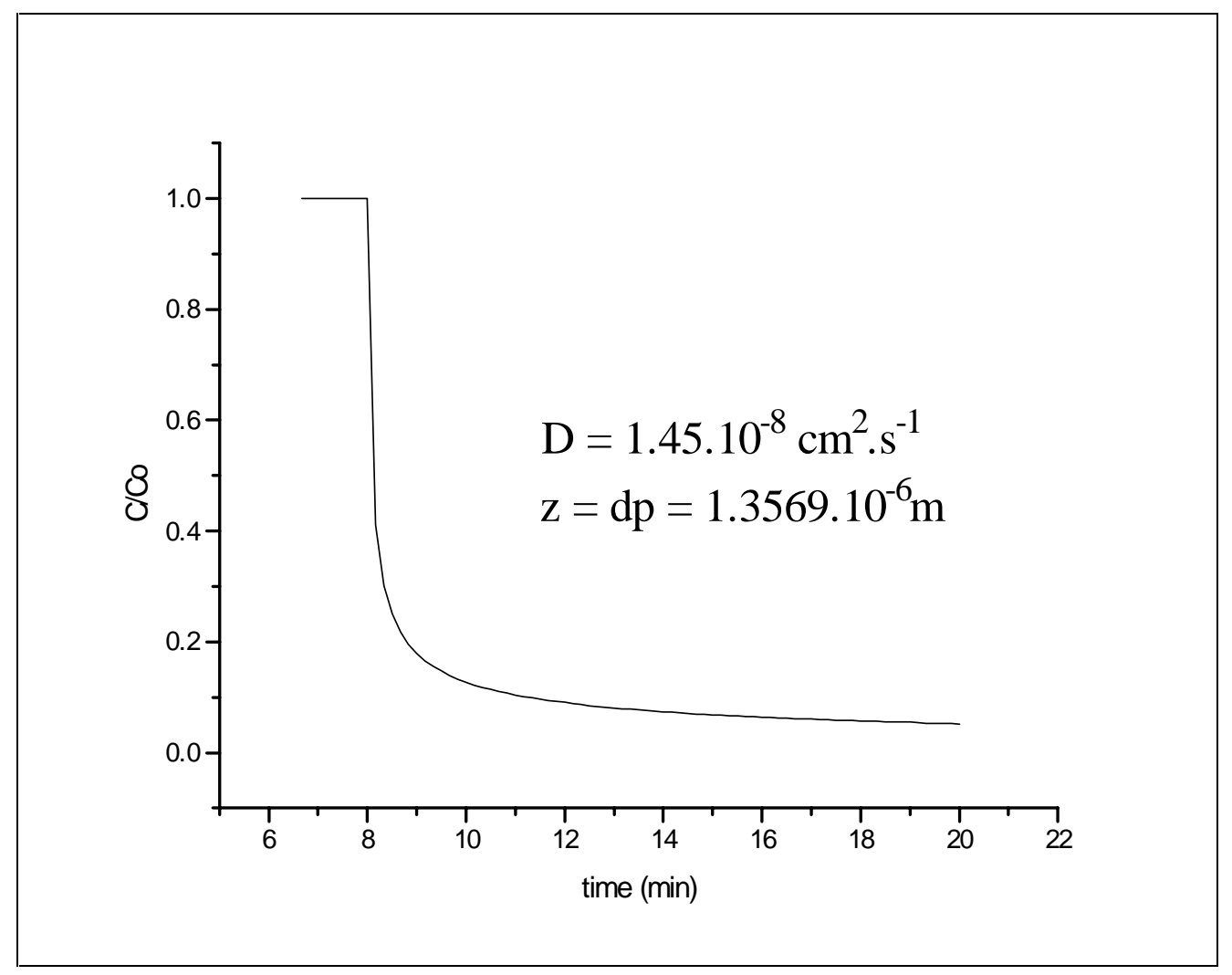

Figure 4.16: Concentration changes of the PVP with time at $\mathrm{T}=100^{\circ} \mathrm{C}$

\subsection{8 - ESTIMATE OF THE INTERPHASE THICKNESS}

An interface represents the thin plane section between two compounds. It is the boundary where properties show a point of discontinuity. On the other hand, an interphase is defined as the region of finite thickness where mechanical and physical gradients occur. The interfacial thickness of a polymer interphase has been derived [149] as follows.

The probability $\mathrm{P}(\mathrm{x}, \mathrm{t})$ that a molecule has diffused to the position $\mathrm{x}$ at time $\mathrm{t}$ is defined as: $P(x, t)=\frac{\partial w(x, t)}{\partial x}$, where $\mathrm{w}$ is the interfacial profile. 
The mean-square interfacial thickness is given by:

$$
d^{2}=\frac{4 \int_{-\infty}^{+\infty}\left(x-x_{0}\right)^{2} \cdot P(x) \cdot d x}{\int_{-\infty}^{+\infty} P(x) \cdot d x}
$$

where $\mathrm{d}$ is the interfacial thickness

$\mathrm{x}_{\mathrm{o}}$ is the coordinate at which $\mathrm{P}(\mathrm{x}, \mathrm{t})$ has the maximum value

$$
\frac{\partial \mathrm{P}\left(\mathrm{x}_{\mathrm{o}}, \mathrm{t}\right)}{\partial \mathrm{x}}=0=\frac{\partial^{2} \mathrm{w}\left(\mathrm{x}_{\mathrm{o}}, \mathrm{t}\right)}{\partial \mathrm{x}^{2}}
$$

Applying those equations to our system, whose concentration profile was given by equation (2.4.o), leads to these results:

$$
\begin{gathered}
P(z, t)=\left(\frac{\partial C(z, t)}{\partial z}\right)=\left(\frac{-2 \cdot C_{0}}{a}\right) \cdot \sum_{n=1}^{\infty} \sin \left(\frac{n \pi b}{a}\right) \cdot \exp \left(\frac{-n^{2} \pi^{2} D \cdot t}{a^{2}}\right) \cdot \sin \left(\frac{n \pi z}{a}\right) \\
\left(\frac{\partial P(z, t)}{\partial z}\right)=\left(\frac{-2 \pi \cdot C_{o}}{a^{2}}\right) \cdot \sum_{n=1}^{\infty} n \cdot \sin \left(\frac{n \pi b}{a}\right) \cdot \exp \left(\frac{-n^{2} \pi^{2} D \cdot t}{a^{2}}\right) \cdot \cos \left(\frac{n \pi z}{a}\right)
\end{gathered}
$$

Since the expressions are quite complicated, another method was considered in order to get a rough estimate of the interphase thickness.

Yukioka et al. [97, 98] reported that the interfacial thickness, d, is given by: $d=2 . \sqrt{\text { D.t }}$, where $\mathrm{D}$ the mutual diffusion coefficient, and $\mathrm{t}$ is the interdiffusion time. In order to have a better understanding of how this equation was obtained, one needs to go back to the original reference cited by Yukioka. This reference paper by Brochard et al. [100] discusses a theory which does not apply to the mutual diffusion coefficient, but to the self-diffusion coefficient. Furthermore, the interfacial thickness was given in this case by the following equation: $\langle d\rangle^{2}=2$. D.t, where $D$ is the self-diffusion coefficient, and $\langle d\rangle^{2}$ is the center-of- 
mass motion. $\langle\mathrm{d}\rangle$ is the overlap distance and corresponds to the average Brownian displacement in time.

Wu et al. [149] stated that, in the case where the interfacial profile is symmetrical, the interfacial thickness is given by the relation: $d=2 \cdot \sqrt{2 . D . t}$, where $d$ is the interfacial thickness and D is the mutual diffusion coefficient. The above equation was actually derived by Crank [102]. Nevertheless, this equation was derived considering both sides of the interface as contributing to the interphase thickness. In the present analysis, this equation would be:

$$
\mathrm{d}=\sqrt{2 . \text { D.t }}
$$

This simple expression applies to a symmetrical profile only, i. e. for the case where the diffusion coefficients for both components are equal. From Table 4.9, one can see that as the temperature increases, the difference between $D_{(P V P)}$ and $D_{(V E)}$ decreases. The temperature at which $\mathrm{D}_{(\mathrm{PVP})}$ and $\mathrm{D}_{(\mathrm{VE})}$ are exactly identical was calculated by the Arrhenius equation with the values of $\mathrm{D}_{\mathrm{o}}$ and $\mathrm{E}_{\mathrm{a}}$ given in Subsection 4.1.6. This temperature was equal to $116.55^{\circ} \mathrm{C} \mp 6^{\circ} \mathrm{C}$ given the $20 \%$ absolute error in determining the diffusion coefficient, and the value of the resulting diffusion coefficient was equal to $6.11 .10^{-8} \mathrm{~cm}^{2} . \mathrm{s}^{-1} \mp 2.36 .10^{-8}$ $\mathrm{cm}^{2} \cdot \mathrm{s}^{-1}$. As previously mentioned in Subsection 4.1.5, the monomer did not yet polymerize at $116.55^{\circ} \mathrm{C}$.

Estimates of the thickness of the polymer interphase based on the Fickian diffusion model yielded values on the order of $60 \mu \mathrm{m}$ after $5 \mathrm{~min}$ at $116.55^{\circ} \mathrm{C}$. The estimate of the interphase thickness is consistent with the result obtained by Dr. Hideko Oyama [144] using Electron Microprobe Analysis (EMP). The thickness was then found to be equal to $30 \mu \mathrm{m}$ when the (PVP/VE) system was polymerized at $150^{\circ} \mathrm{C}$ (the diffusion time was roughly estimated to be 5 min during this process). 


\section{2 - MOLECULAR INTERACTIONS}

In addition to quantitative information concerning concentration variations, the FTIR-ATR technique also allows one to characterize molecular interactions occuring within the (PVP/VE) system.

This section begins by giving an introduction to the physical principles of the hydrogen bond. Then, in the following subsection, evidence of hydrogen bonding is pointed out, in the carbonyl and the hydroxyl regions of the (PVP/VE) sample, as well as in the selfassociation of the VE. An attempt is made in the last subsection to calculate the equilibrium constant of hydrogen bond formation for this system.

\subsection{1 - THE HYDROGEN BOND}

Hydrogen bonding interactions occuring between the PVP and the VE were examined by ATR spectroscopy. Indeed, it is well known that the amide carbonyl group of the PVP is a strong hydrogen bond acceptor $[132,150]$. Figure 4.17 shows the hydrogen bond interaction between the PVP and the VE.

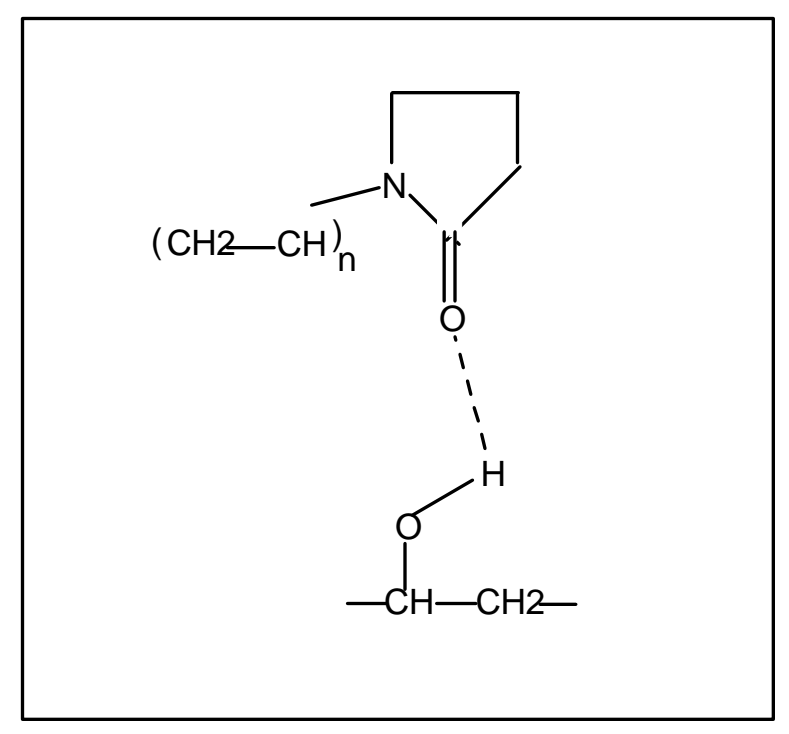

Figure 4.17: Hydrogen bond interaction between the PVP and the VE 
Hydrogen bonding, a particular type of molecular interaction, occurs when a hydrogen atom lies in between two highly electronegative atoms such as $\mathrm{O}, \mathrm{F}$ and $\mathrm{N}$, as suggested in Figure 4.18. Hydrogen bonds are dynamic bonds: they break and reform continuously. The mean lifetime of a hydrogen bond is on the order of $10^{-11}$ seconds [151]. Hydrogen bond is sufficient to hold two molecules together so that they behave as one unit. However, its strength is quite weak: 4 to $40 \mathrm{~kJ} / \mathrm{mol}$ [151]. For comparison, covalent bonds have strengths of the order of $200 \mathrm{~kJ} / \mathrm{mol}$ and Van der Waals attractions of the order of 0.8 $\mathrm{kJ} / \mathrm{mol}$.

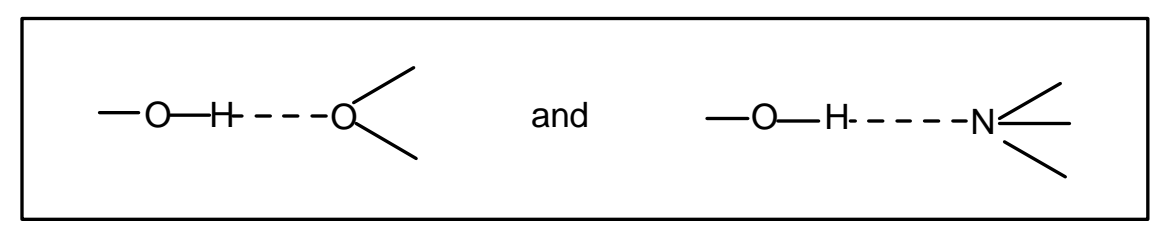

Figure 4.18: Hydrogen bonding

Since the strength of the hydrogen bond O---H is relatively weak, its stretching mode appears at very low frequencies (or very low wavenumbers since the frequency is directly proportional to the wavenumber), and can be measured only in the far-infrared region ( from $400 \mathrm{~cm}^{-1}$ to $10 \mathrm{~cm}^{-1}$ ). However, the analysis of the carbonyl $\mathrm{C}=\mathrm{O}$ stretching region $\left(1750 \mathrm{~cm}^{-1}\right.$ to $\left.1600 \mathrm{~cm}^{-1}\right)$ and also of the hydroxyl $\mathrm{OH}$ stretching region (between $3500 \mathrm{~cm}^{-1}$ and $3000 \mathrm{~cm}^{-1}$ ) in the mid-infrared region can give us information about the nature of the hydrogen bond. Hydrogen bond formation results in a decrease of the strength of the $\mathrm{C}=\mathrm{O}$ and $\mathrm{OH}$ stretching vibrations and thus the bands associated with the hydrogen bonded $\mathrm{C}=\mathrm{O}$ and $\mathrm{OH}$ groups appear at lower wavenumbers than the bands associated with the free $\mathrm{C}=\mathrm{O}$ and $\mathrm{OH}$ groups. As a reminder, the vibrational frequency of a diatomic molecule A-B in simple harmonic motion is given by the expression [107]: 


$$
\gamma=\left(\frac{1}{2 \pi}\right) \cdot \sqrt{f_{A B}\left(\frac{M_{A}+M_{B}}{M_{A} \cdot M_{B}}\right)}
$$

where $\gamma$ is the frequency in $\mathrm{s}^{-1}$

$\mathrm{M}_{\mathrm{i}}$ is the atomic weight of the atom $\mathrm{i}$

$f_{A B}$ is the force constant of the $A B$ bond

Thus, by measuring the band shifts in the $\mathrm{C}=\mathrm{O}$ and $\mathrm{OH}$ bands, it is possible to evaluate the average strength of the hydrogen bond.

\subsection{2 - EVIDENCE OF HYDROGEN BONDING}

Hydrogen bonding interactions occuring in the (PVP/VE) system were characterized by ATR spectroscopy. These interactions may have some implications on the diffusion coefficient and the interphase thickness.

Hydrogen bonding between the PVP and the VE was clearly observed in the carbonyl region. Self-association of the VE was also evident. The unavoidable presence of moisture makes the evaluation of hydrogen bond interactions between the PVP and the VE in the hydroxyl region difficult.

\subsubsection{1 - The Carbonyl Region}

During annealing of the $(\mathrm{PVP} / \mathrm{VE})$ sample at $\mathrm{T}=100^{\circ} \mathrm{C}$, the $\mathrm{C}=\mathrm{O}$ stretching region of the PVP underwent very drastic peak shifts and intensity changes, as shown in Figure 4.19. The $\mathrm{C}=\mathrm{O}$ band of the pure $\mathrm{PVP}$ at $\mathrm{T}=100^{\circ} \mathrm{C}$ occured at $1669 \mathrm{~cm}^{-1}$. This wavenumber is quite low for a carbonyl band, but this is due to the fact that this band contains not only contributions from $\mathrm{C}=\mathrm{O}$ stretching vibrations, but also N-C stretching contributions [150]. While studying the diffusion, another band appeared at $1650 \mathrm{~cm}^{-1}$ at $\mathrm{t}=8 \mathrm{~min}$, time for which the VE reached the penetration depth at $\mathrm{T}=100^{\circ} \mathrm{C}$. A substantial shift to lower wavenumbers occured, which could be attributed to intermolecular hydrogen bonding, or 
inter-association, between the $\mathrm{OH}$ groups of the VE and the carbonyl groups of the PVP $(\mathrm{C}=\mathrm{O}---\mathrm{OH})$.

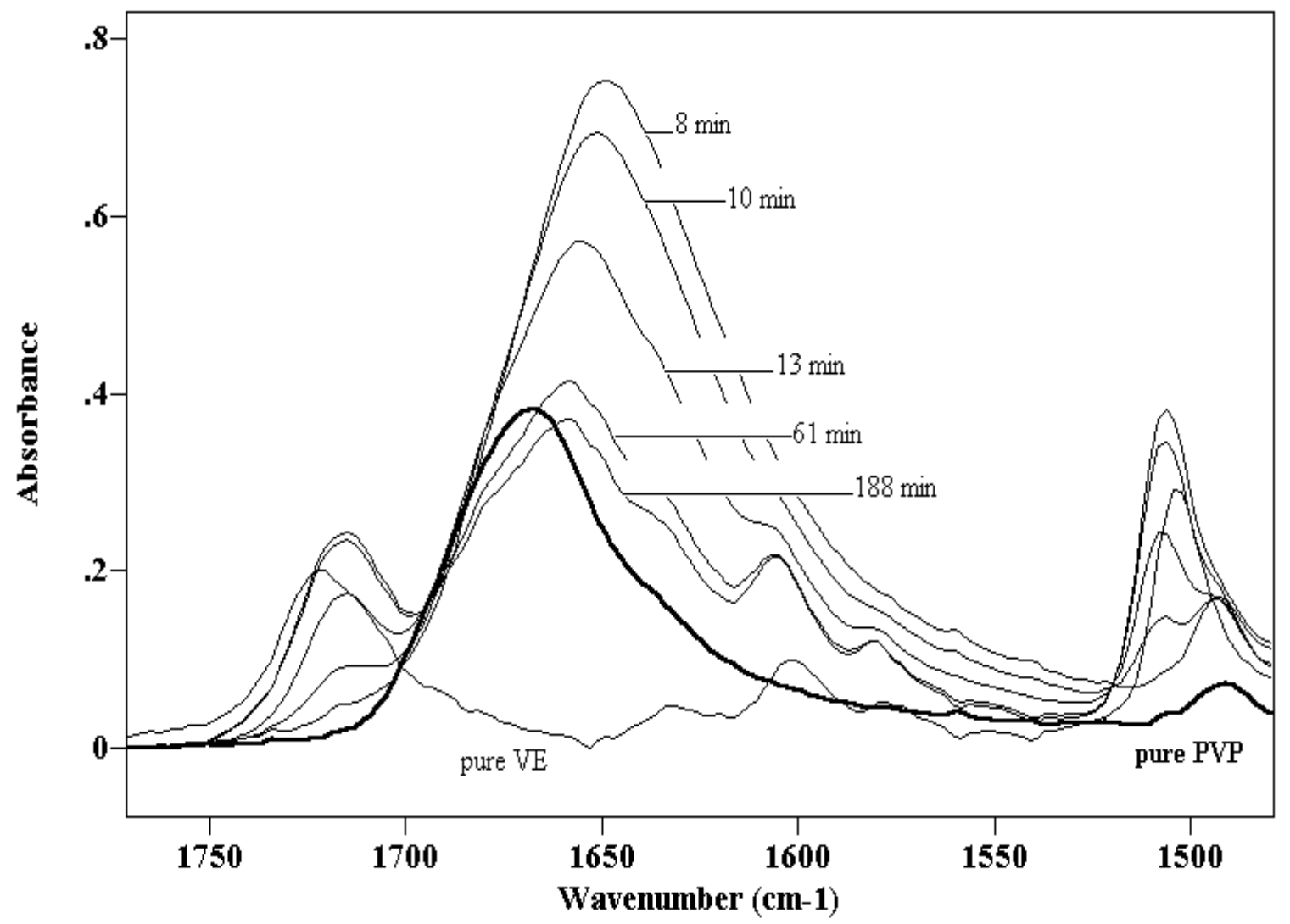

Figure 4.19: Time evolution spectra in the carbonyl region at $\mathrm{T}=100^{\circ} \mathrm{C}$

The strength of the hydrogen bond has been related to the magnitude of the frequency shift [151]. This information can be found in Table 4.11. Since the shift was about $20 \mathrm{~cm}^{-1}$ in the present case, the hydrogen bonds can be qualified as quite weak. 
Table 4.11: Frequency shifts upon hydrogen bond formation (from [151])

\begin{tabular}{|l|r|r|l|}
\hline \multicolumn{1}{|c|}{ strength of } & IR frequency & enthalpy of the bond & examples \\
\hline hydrogen bond & shift $\left(\mathrm{cm}^{-1}\right)$ & $(\mathrm{kcal} / \mathrm{mol})$ & \\
\hline weak & 10 to 50 & 1 & PVC-Polyesters \\
\hline medium & 300 & 5 & $-\mathrm{OH}$, amide, urethane \\
\hline intermediate & 600 & 6 to 8 & -COOH \\
\hline strong & 800 to 2000 & $>8$ & acid salts \\
\hline
\end{tabular}

\subsubsection{2 - Self-Association}

The VE may also self-associate since its structure, shown in Figure 3.2, contains functional groups which hydrogen bond to one another. The carbonyl and the hydroxyl groups of the VE ( $\mathrm{C}=\mathrm{O}---\mathrm{OH})$ and the hydroxyl groups alone ( $\mathrm{HO}---\mathrm{OH}$ ) could interact via the formation of weak hydrogen bonds. The self-association could lead to the formation of linear or cyclic complexes.

In order to check the assumption of self-association, the variation of the spectrum of the VE monomer itself with temperature was followed by ATR spectroscopy. Studying the change in the infrared spectrum with temperature can provide useful information about the nature of the interactions.

As shown in Figure 4.20, two bands could be identified in the $\mathrm{C}=\mathrm{O}$ stretching frequency region: one band centred at $1722 \mathrm{~cm}^{-1}$ attributed to the free carbonyl groups, and another band at $1717 \mathrm{~cm}^{-1}$ assigned to the hydrogen bonded carbonyl groups. Evidence of selfassociation was thus observed. It implies that all the $\mathrm{OH}$ groups of the VE interact not only with the $\mathrm{C}=\mathrm{O}$ groups of the PVP, but also with those of the VE. As the temperature increased, the intensity of the free band increased compared to that of the hydrogen bonded band. The strength of the hydrogen bond decreased with temperature. Coleman et al. [151] explained that as the thermal motion increases, the volume of the polymer and the average intermolecular distance between chains increases, leading to the decrease of the strength of the hydrogen bond. 


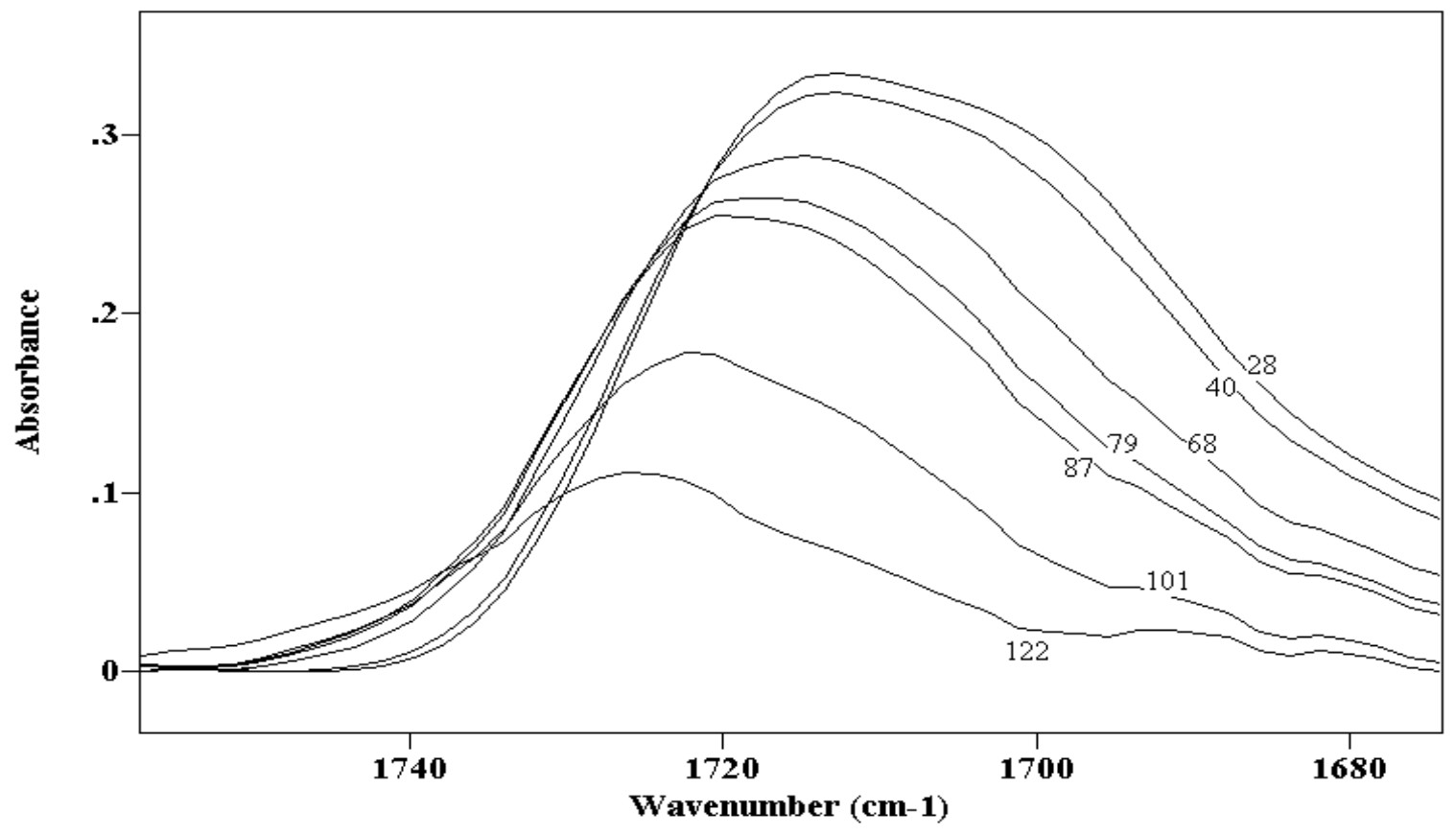

Figure 4.20: Influence of the temperature $\left({ }^{\circ} \mathrm{C}\right)$ on the pure VE in the carbonyl region

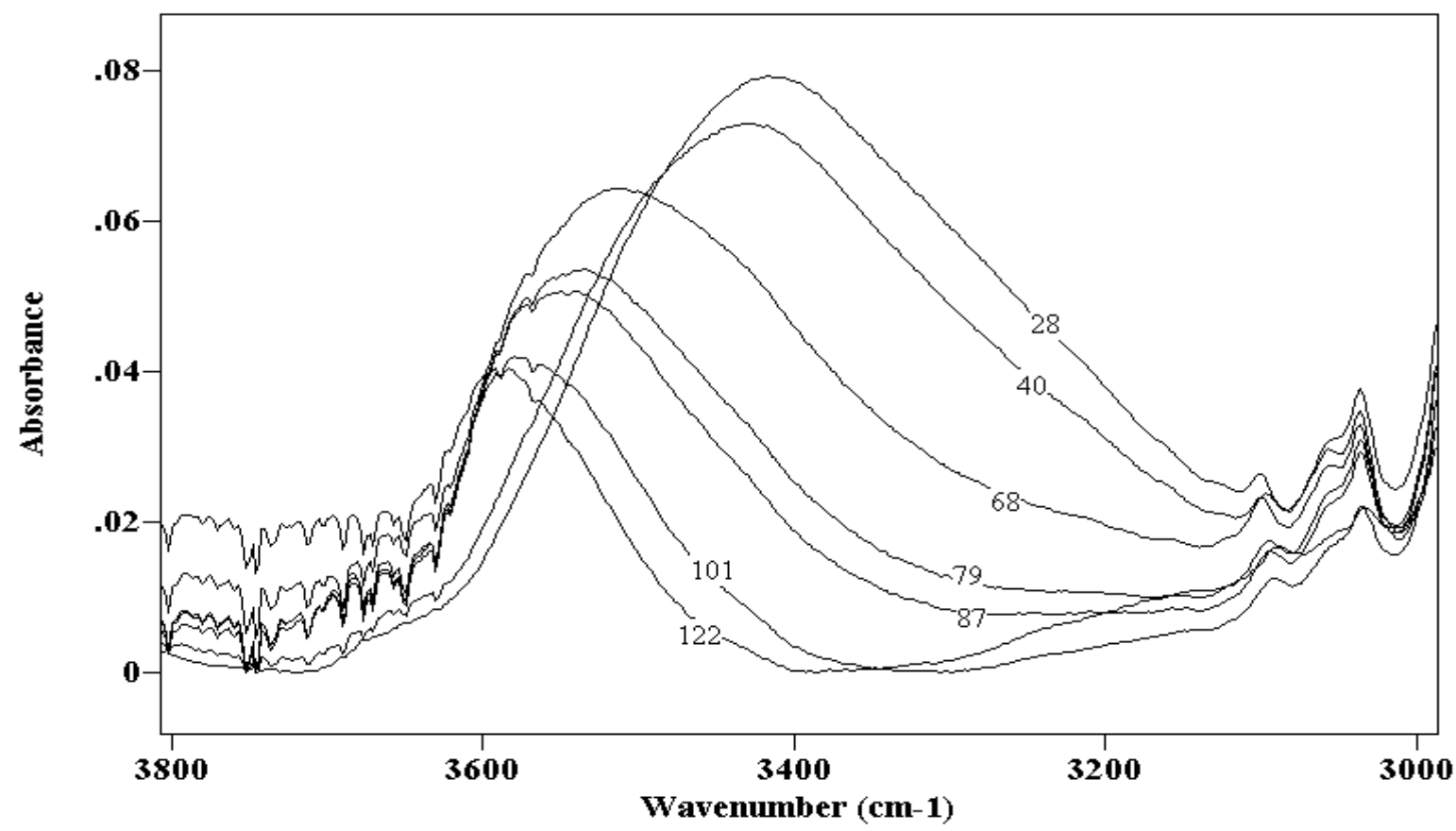

Figure 4.21: Influence of the temperature $\left({ }^{\circ} \mathrm{C}\right)$ on the pure VE in the hydroxyl region 
As shown in Figure 4.21, a free hydroxyl band was located in the $\mathrm{OH}$ stretching frequency region at $3512 \mathrm{~cm}^{-1}$, and a hydrogen bonded hydroxyl band at $3418 \mathrm{~cm}^{-1}$. The $\mathrm{OH}$ groups could interact with $\mathrm{OH}$ as well as $\mathrm{C}=\mathrm{O}$ groups. Unfortunately, identification of the position of the bands by using the second derivative analysis has actually failed. As the temperature increased, the strength of the hydrogen bond decreased with temperature again.

No significant changes in the peak position or the shape of the bands of the pure PVP was observed with temperature by ATR spectroscopy, as illustrated in Figure 4.22. Since the nitrogen atom of the PVP is not linked directly with a hydrogen atom, no hydrogen bonding interactions are possible with the carbonyl groups. Therefore, the pure PVP cannot self-associate and the band characteristic of the carbonyl groups remains constant with increasing temperature.

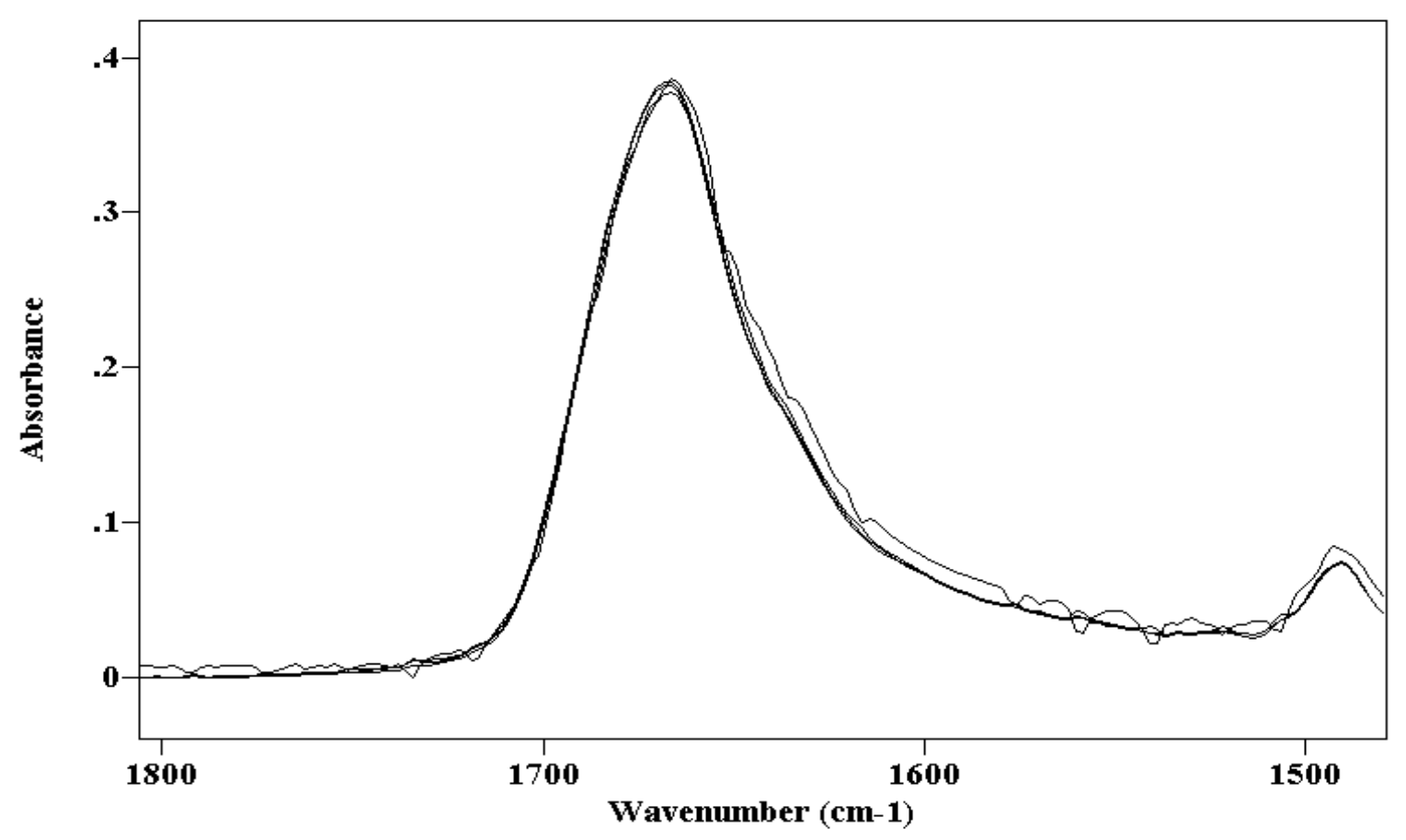

Figure 4.22: Influence of temperature (from $45^{\circ} \mathrm{C}$ to $120^{\circ} \mathrm{C}$ ) on the pure PVP in the carbonyl region 
However, infrared transmission experiments suggested that moisture influences the position of the carbonyl band of the pure PVP, as shown in Figures 4.23 and 4.24. The more moisture in the PVP, the more hydrogen bond interactions, and the lower the wavenumber in the carbonyl region.

In conclusion, the PVP cannot self-associate but has a functional group capable of forming hydrogen bonds with the other component, VE. The second component, the VE, can hydrogen bond to itself in the pure state since it had both donor and acceptor groups. One would expect that these interactions would also influence the diffusion mechanism of the two components in the interphase. Some possible explanations of hydrogen bonding participation during interdiffusion are offered in the discussion section of this thesis.

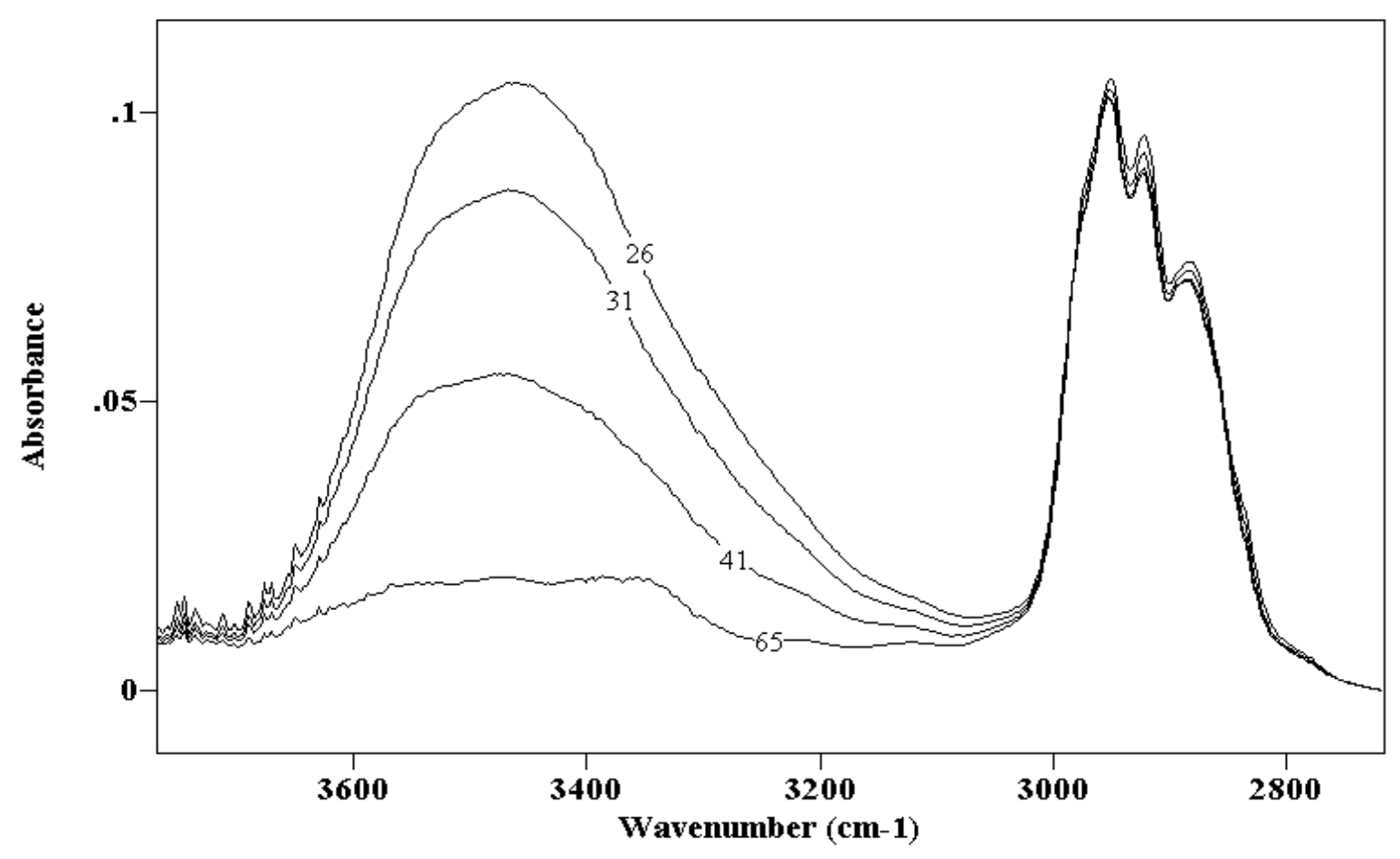

Figure 4.23: Influence of moisture on the pure PVP in the hydroxyl region (The moisture content decreased as the temperature increased from 26 to $65^{\circ} \mathrm{C}$ ) 


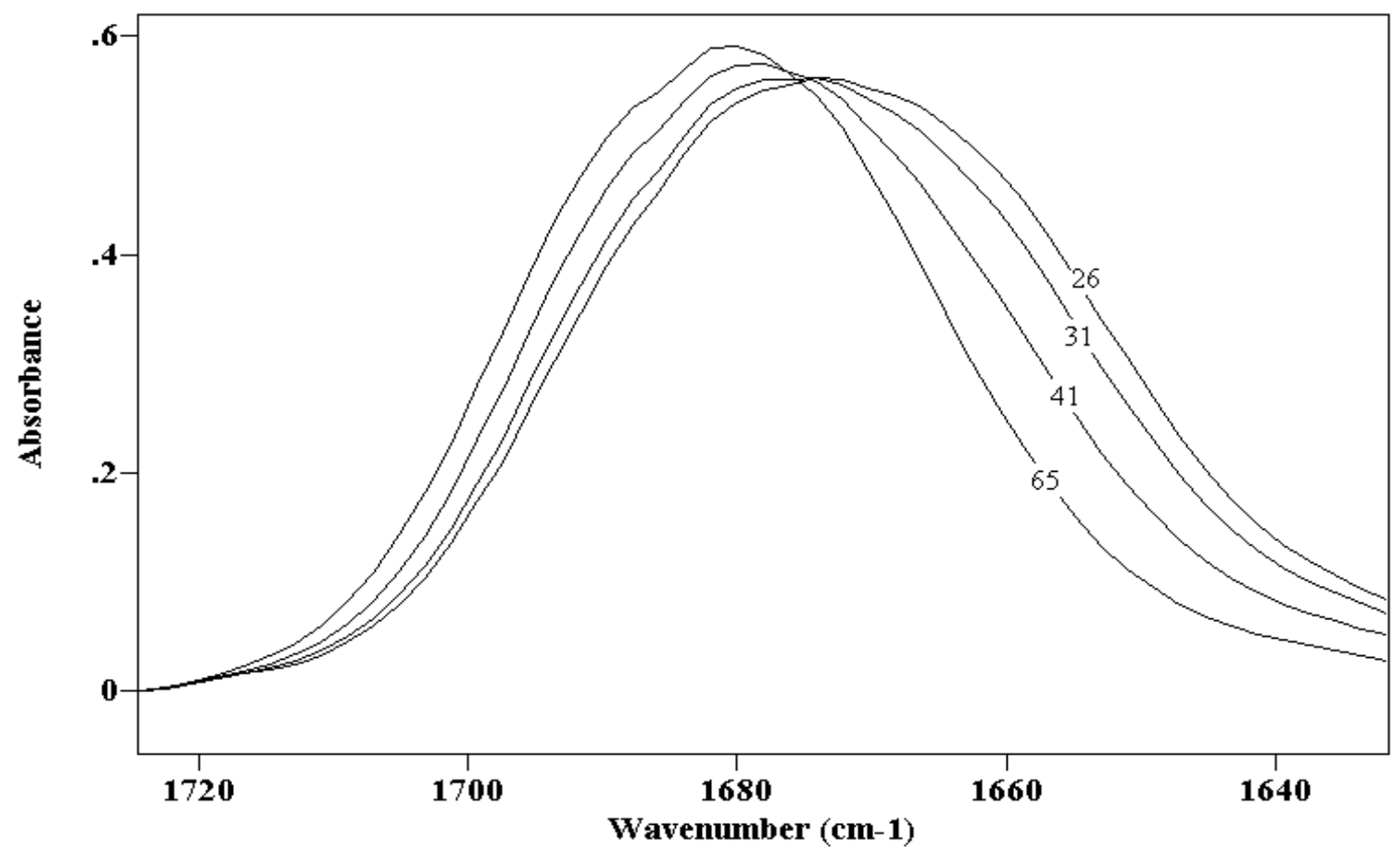

Figure 4.24: Influence of moisture on the pure PVP in the carbonyl region (The moisture content decreased as the temperature increased from 26 to $65^{\circ} \mathrm{C}$ )

\subsubsection{3 - The Hydroxyl Region}

When following the diffusion between the PVP and the VE at $\mathrm{T}=100^{\circ} \mathrm{C}$ by ATR spectroscopy by looking at the hydroxyl region, three bands were distinguished: an unassociated $\mathrm{OH}$ band at $3512 \mathrm{~cm}^{-1}$ and a self-associated $\mathrm{OH}$ band at $3418 \mathrm{~cm}^{-1}$, as stated in Subsection 4.2.2.2, and also a hydrogen bonded $\mathrm{OH}$ band at $3230 \mathrm{~cm}^{-1}$. Figure 4.25 shows the evolution in the hydroxyl region at $\mathrm{T}=100^{\circ} \mathrm{C}$.

Nevertheless, moisture was probably present in the system since the areas of the bands located between $3600 \mathrm{~cm}^{-1}$ and $3100 \mathrm{~cm}^{-1}$ decreased with time instead of increasing with time, as it should do with the intrusion of the VE. Therefore, one cannot exclude the possibility that the hydrogen bonded $\mathrm{OH}$ could be attributed to $\mathrm{H}_{2} \mathrm{O}$. 


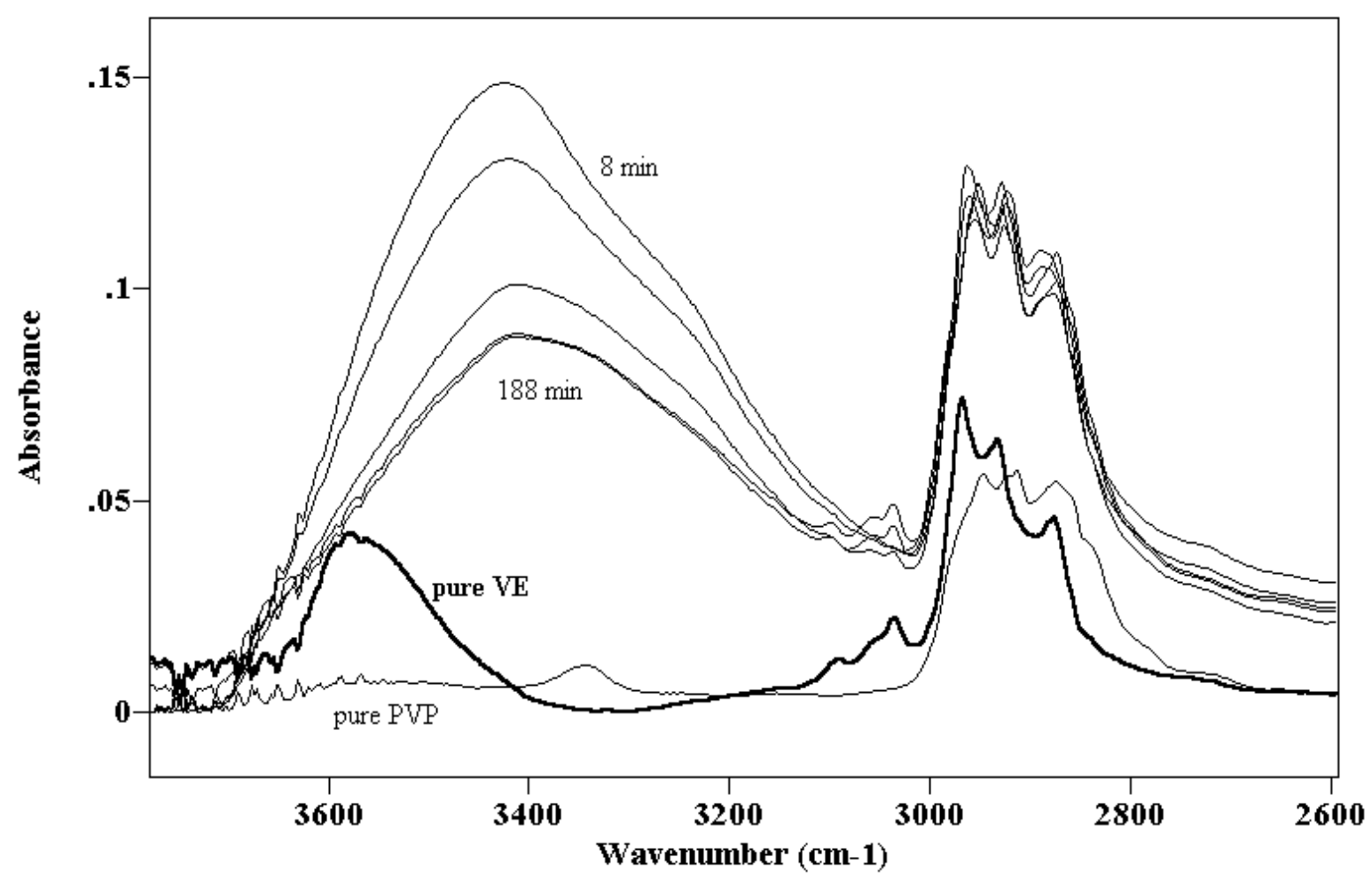

Figure 4.25: Time evolution spectra in the hydroxyl region at $\mathrm{T}=100^{\circ} \mathrm{C}$ The interdiffusion times (in minutes) are: 8, 10, 13, 61 and 188

\subsection{3 - THE EQUILIBRIUM CONSTANT OF HYROGEN BOND FORMATION}

As developed in Subsection 4.2.2, hydrogen bonding is occuring between the two components of the (PVP/VE) system. This interaction will have some effects on the value of the diffusion coefficient. In order to see how the hydrogen bonding affects the activation energy for diffusion, it would be interesting to evaluate the enthalpy of the system. This enthalpy can be calculated from the value of the equilibrium constant of the (PVP/VE) system. 
The equilibrium for the formation of a hydrogen bond is represented by:

$$
[\mathrm{C}=\mathrm{O}]+[\mathrm{OH}] \leftrightarrow[\mathrm{C}=\mathrm{O}---\mathrm{OH}]
$$

The association equilibrium constant $\mathrm{K}$ is then given by:

$$
\mathrm{K}=\frac{[\mathrm{C}=\mathrm{O}---\mathrm{OH}]}{[\mathrm{C}=\mathrm{O}] \cdot[\mathrm{OH}]}
$$

At equilibrium, the Gibbs free energy $\Delta \mathrm{G}$ is defined as:

$$
\Delta \mathrm{G}=-\mathrm{RT} .(\ln \mathrm{K})=\Delta \mathrm{H}-\mathrm{T} \cdot \Delta \mathrm{S}
$$

Thus

$$
\ln \mathrm{K}=\left(\frac{\Delta \mathrm{S}}{\mathrm{R}}\right)-\left(\frac{1}{\mathrm{~T}}\right) \cdot\left(\frac{\Delta \mathrm{H}}{\mathrm{R}}\right)
$$

By plotting $(\operatorname{lnK})$ versus $(1 / \mathrm{T})$, the entropy $\Delta \mathrm{S}$ can be obtained from the intercept, and the enthalpy $\Delta \mathrm{H}$ from the slope.

The fraction of hydrogen bonded carbonyl groups $\mathrm{f}_{\mathrm{HB}}^{\mathrm{C}=\mathrm{O}}$ has to be determined in order to calculate K. Assuming that the Beer-Lambert law (equation 2.4.a) is valid, the fraction of hydrogen bonded carbonyl groups $\mathrm{f}_{\mathrm{HB}} \mathrm{C}=\mathrm{O}$ is equal to [151]:

$$
\mathrm{f}_{\mathrm{HB}}{ }^{\mathrm{C}=\mathrm{O}}=\frac{\mathrm{A}_{H B}}{\mathrm{~A}_{H B}+\alpha \cdot \mathrm{AF}_{\mathrm{F}}}
$$


where $\mathrm{f}_{\mathrm{HB}}^{\mathrm{C}=\mathrm{O}}$ is the fraction of hydrogen bonded carbonyl groups

$A_{F}$ is the area of the band associated to the free carbonyl groups

$\mathrm{A}_{\mathrm{HB}}$ is the area of the band associated to the hydrogen bonded carbonyl groups

$\alpha$ is the ratio of the absorptivities of the hydrogen bonded band to the one of the free band, and $\alpha=\left(\varepsilon_{\mathrm{HB}} / \varepsilon_{\mathrm{F}}\right)$.

The ratio of the absorptivities, $\alpha$, can be determined by varying the temperature of the sample, assuming that these absorption coefficients remain constant over the range of temperature tested. It has been shown [151] that: $\left(\mathrm{A}_{\mathrm{HB}}\right)_{\mathrm{T}}+\alpha \cdot\left(\mathrm{A}_{\mathrm{F}}\right)_{\mathrm{T}}=$ constant. Thus by collecting the spectra of a sample at different temperatures and plotting $\mathrm{A}_{\mathrm{HB}}$ as a function of $A_{F}$ for each temperature, the slope will give us $-\alpha$.

A solution of 50/50 composition by weight of (PVP/VE) was diluted in methanol. A thin film of solution was casted on a $\mathrm{KBr}$ window for FTIR transmission spectroscopy. In order to remove any residual solvent, the sample was first dried slowly at room temperature and then placed in a vacuum oven for one day. The spectrum of the methanol $\mathrm{CH}_{3} \mathrm{OH}$ was found in the literature [152], and it was verified that no characteristic bands of methanol appeared on the spectrum of the blend. Transmission spectroscopy was used to monitor the chemical changes of the blend, for temperatures ranging from $50^{\circ} \mathrm{C}$ to $81^{\circ} \mathrm{C}$. ATR spectroscopy could not be used as a technique in this analysis since (4.2.c) was derived assuming the Beer-Lambert law for transmission. The expression of the fraction of hydrogen bonded carbonyl groups could be derived for ATR spectroscopy using (2.4.1), but diffusion within the penetration depth would complicate the analysis.

The carbonyl region between $1765 \mathrm{~cm}^{-1}$ and $1590 \mathrm{~cm}^{-1}$, shown in Figure 4.26, was curvefitted with four peaks, namely $1722 \mathrm{~cm}^{-1}, 1690 \mathrm{~cm}^{-1}, 1650 \mathrm{~cm}^{-1}$ and $1607 \mathrm{~cm}^{-1}$. The peak at $1690 \mathrm{~cm}^{-1}$ corresponded to the free groups of the PVP whereas the peak at $1650 \mathrm{~cm}^{-1}$ corresponded to the hydrogen bonded groups. It was determined in Subsection 4.2.2.1 that 
the $\mathrm{C}=\mathrm{O}$ band of the pure PVP occured at $1670 \mathrm{~cm}^{-1}$. A shift of the carbonyl band to higher frequencies is expected for transmission spectroscopy, compared to ATR spectroscopy [153].

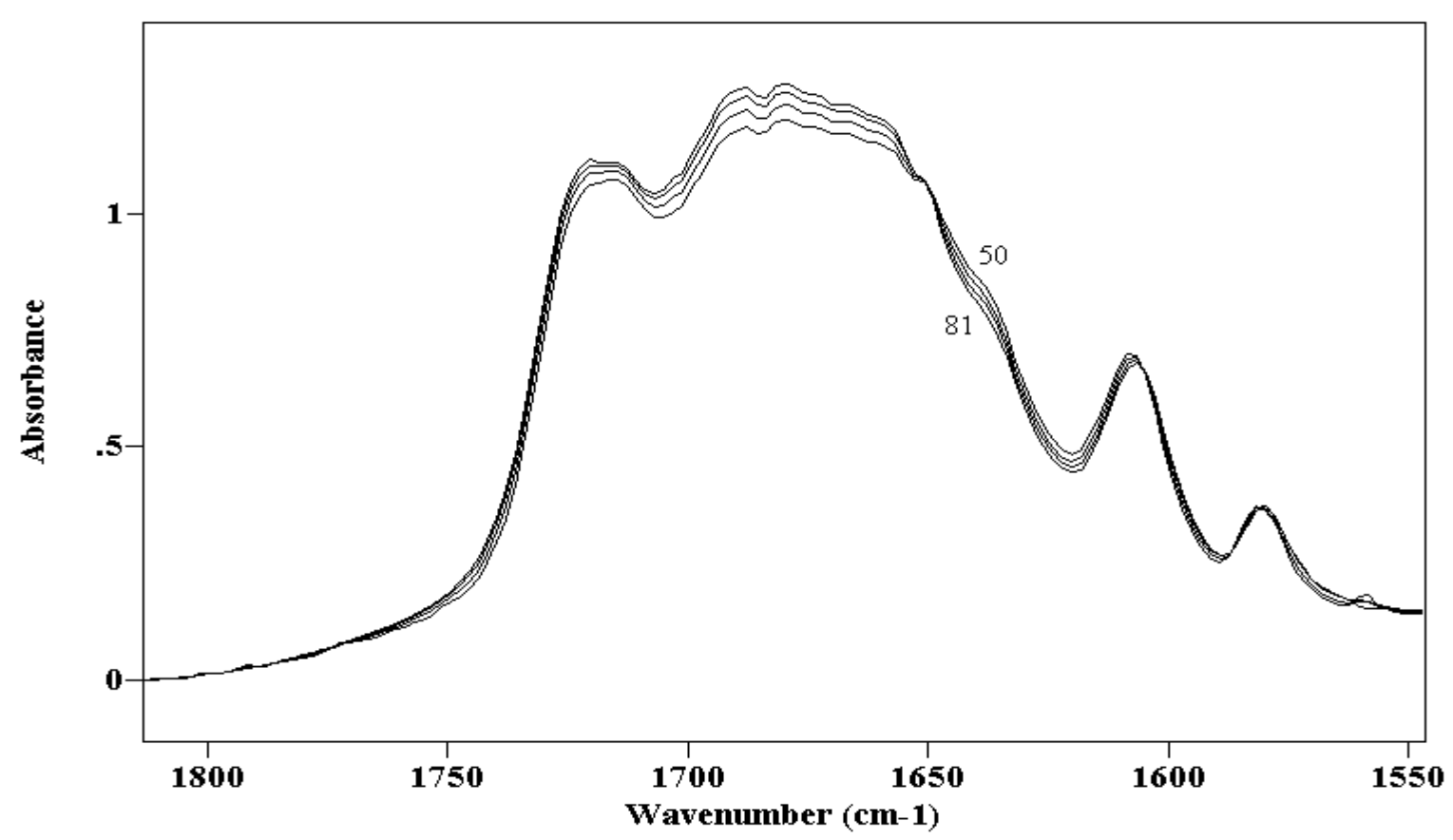

Figure 4.26: Evolution of a 50/50 blend with temperature in the carbonyl region The temperatures (in ${ }^{\circ} \mathrm{C}$ ) are: 50, 60, 70 and 81

The areas of the bands associated with the stretching of the free and the hydrogen bonded carbonyl groups of the PVP were determined by curvefitting. Figure 4.27 shows $A_{H в}$ as a function of $A_{F}$. Unfortunately, the slope gave us $\alpha=0.7$. Usually the ratio is equal to 1.2 for the carbonyl bond of an amide [151]. Since the ratio has to be greater than 1 [151], it was impossible to pursue the analysis. Further analysis would indeed lead to a negative entropy. The error may have had origin in the subjective nature of the curvefitting procedure. Nevertheless, it is clear that hydrogen bonding interactions between PVP and VE play a 
significant role in the development of the interphase and provide interphase-matrix adhesion.

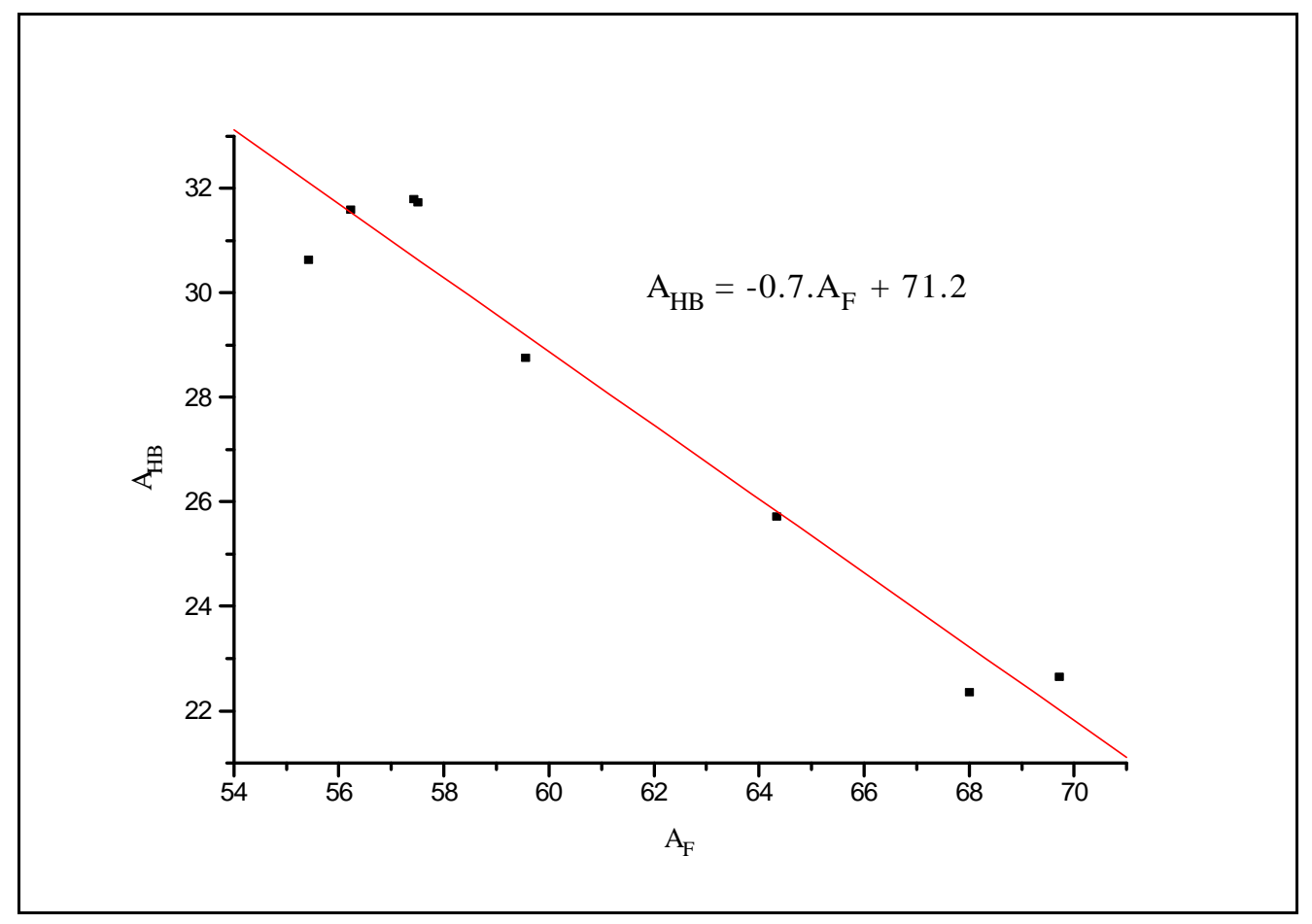

Figure 4.27: $\mathrm{A}_{\mathrm{HB}}$ as a function of $\mathrm{A}_{\mathrm{F}}$

\section{3 - DISCUSSION}

Now that the experimental results have been provided, the next step is to discuss the errors involved in the diffusion model and to suggest some parameters which have not been taken into account in the investigation. 


\subsection{1 - DISCUSSION OF THE ERRORS INVOLVED IN THE MODEL}

This subsection begins with evaluating the experimental errors and concludes by discussing the theoretical assumptions made in formulating the diffusion model (2.4.s).

\subsubsection{1 - Experimental Errors}

The parameters leading to experimental errors are the thicknesses of the polymer and the monomer, the refractive indices of the various components, the wavenumber, the penetration depth, the time, the temperature, the height of the bands and the computer analysis. Since it was impossible to evaluate the errors by manipulating the equation (2.4.s), the crucial parameters had to be evaluated independently.

The values of the refractive indices of the ZnSe crystal and the PVP, $n_{1}$ and $n_{2}$ respectively, were found in the literature, as already stated in Subsection 3.1.3. However, it is well established that the index of refraction of a material changes with frequency and temperature $[154,155,156]$. No mention of those parameters was made with respect to the value of $n_{1}[110,112,113,114]$, while the value of $n_{2}$ was obtained for $\lambda=5893 \AA$ at an unreported temperature [135].

The measure of the heights of the peaks characteristic of the VE as a function of time generated some errors. As mentioned in Subsection 3.4.4, the number of peaks, the shapes of the peaks, their positions, and their widths at half height had to be estimated in the curvefitting program developed for the GRAMS software. Furthermore, two different initial

guesses gave two different results, which were within 3\%. The height of the carbonyl band of the PVP was measured with respect to the highest intensity point of the band, and not at a constant wavenumber. However, this peak shifted by three wavenumbers as the diffusion was going on.

In order to fit the absorbance-time data to the Fickian diffusion model (2.4.s), the starting time had to be adjusted by substracting a lag time from the actual start time of the experiment, as explained in Subsection 4.1.3. This lag time was obtained by extrapolating the plot of the peak heights of the VE versus time (as shown in Figure 4.8) to zero height. 
However, it should be pointed out that the absorbance of the PVP decreased even before the adjusted starting time. This may be due to the fact that the carbonyl peak characteristic of the PVP, occured at lower wavenumbers than the VE carbonyl peak, as summarized in Table 4.3. However, since the penetration depth is higher at lower wavenumbers, as illustrated in Figure 2.5, it is possible that the light penetrated further into the PVP sample and changes in the PVP spectra were observed before changes in the VE spectra.

There was considerable uncertainty in the assignment of the effective film thickness of the VE. Since the surface of the rigid VE was not flat, an average value for the thickness was taken. Furthermore, the VE softened as the temperature increased and, as a consequence, the thickness changed. Nevertheless, it was noticed by changing the value of the thickness in the Fortran program "diff.for", given in Appendix C, that the value of the diffusion coefficient did not vary significantly. An experimental error of $43 \%$ in the thickness of the VE led to an error of only $0.7 \%$ at $100^{\circ} \mathrm{C}$ in the value of the diffusion coefficient, determined with respect to the peak at $1422 \mathrm{~cm}^{-1}$. However, the thickness of the PVP measured via profilometry was a crucial parameter. An experimental error of $17 \%$ in the thickness measurement corresponded to an error of $29 \%$ in the value of the diffusion coefficient.

The determination of the thickness of the PVP was the major source of uncertainties. In order to verify theoretically the value of the thicknesses of the PVP and the VE, results obtained by ATR spectroscopy can be used. This analysis is discussed below.

On one hand, one can use equations (2.4.r) and (2.4.u), which relate the absorbance values to the PVP thickness, to calculate $\left(\mathrm{A}_{\infty}-\mathrm{A}_{\mathrm{o}}\right)$ for the PVP and the VE respectively, and to compare this difference with the experimental data. However, the difference between $\mathrm{A}_{\propto}$ and $\mathrm{A}_{\mathrm{o}}$ could not be predicted from the mathematical equations (2.4.r) and (2.4.u) since parameters such as $\mathrm{C}_{\mathrm{o}}$ and $\mathrm{C}_{\mathrm{mo}}$ were unknown. Nevertheless, for a given peak, it was verified that, the experimental difference $\left(\mathrm{A}_{\propto}-\mathrm{A}_{\mathrm{o}}\right)$ remained almost constant with temperature, as demonstrated in Figure 4.28. This difference was positive for the VE and negative for the PVP, as suggested by the equations (2.4.r) and (2.4.u). 


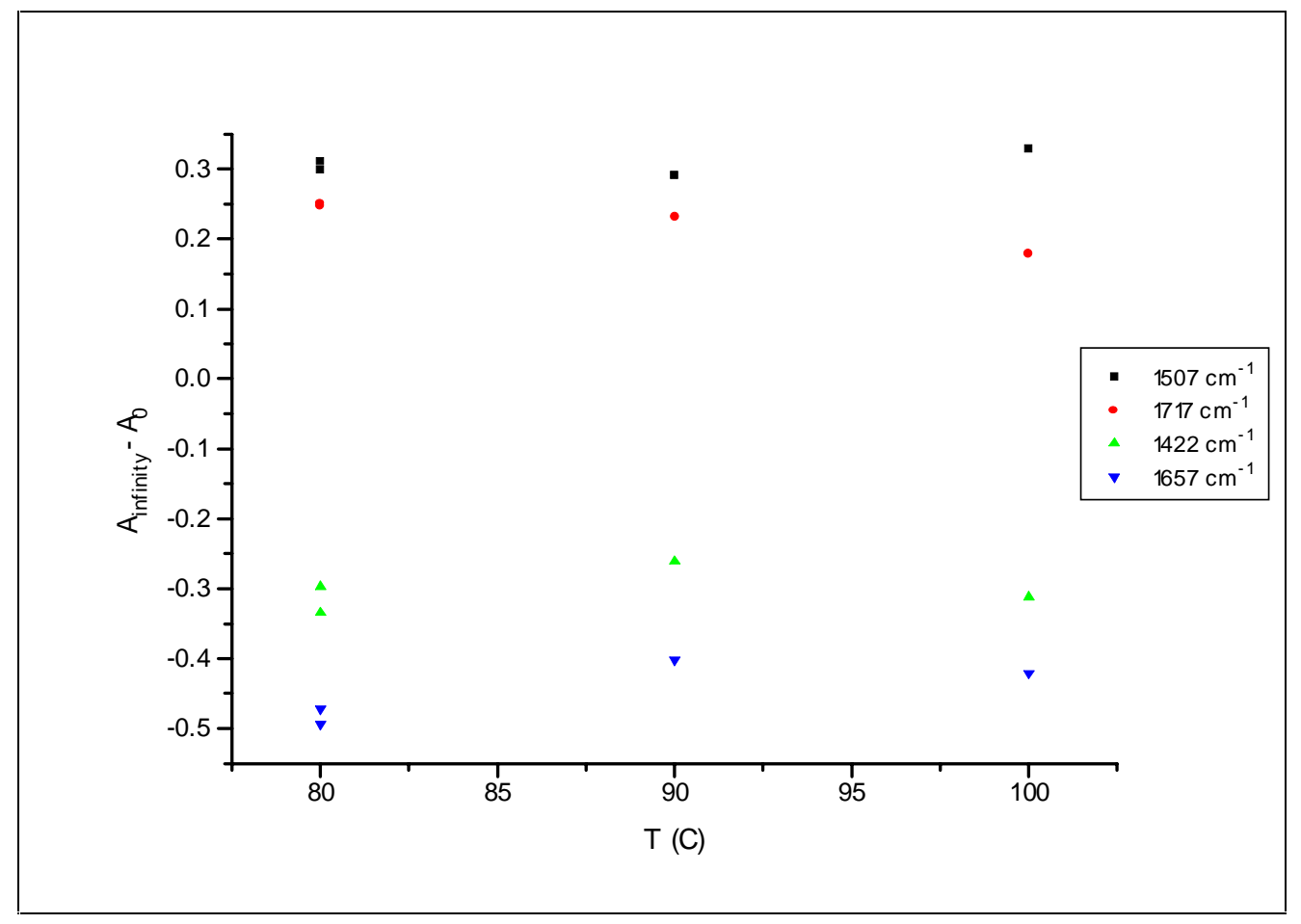

Figure 4.28: Experimental values of $\left(\mathrm{A}_{\propto}-\mathrm{A}_{\mathrm{o}}\right)$ versus temperature

On the other hand, the ratios $\left(\mathrm{A}_{\propto} / \mathrm{A}_{\mathrm{o}}\right)$ for the PVP and the VE could also be determined by the equations (2.4.q) and (2.4.t), respectively. Since initially the VE was outside the penetration depth, $\mathrm{A}_{\mathrm{o}}$ was equal to zero for this particular component. It was thus impossible to obtain the ratio $\left(\mathrm{A}_{\propto} / \mathrm{A}_{\mathrm{o}}\right)$ for the VE. Experimental values of $\left(\mathrm{A}_{\propto} / \mathrm{A}_{\mathrm{o}}\right)$ based on the two peaks characteristic of the PVP were plotted as a function of temperature, as shown in Figure 4.29. Contrary to what was expected by (2.4.q), the ratio $\left(\mathrm{A}_{\circ} / \mathrm{A}_{\mathrm{o}}\right)$ did not remain constant for a given band of the PVP. 


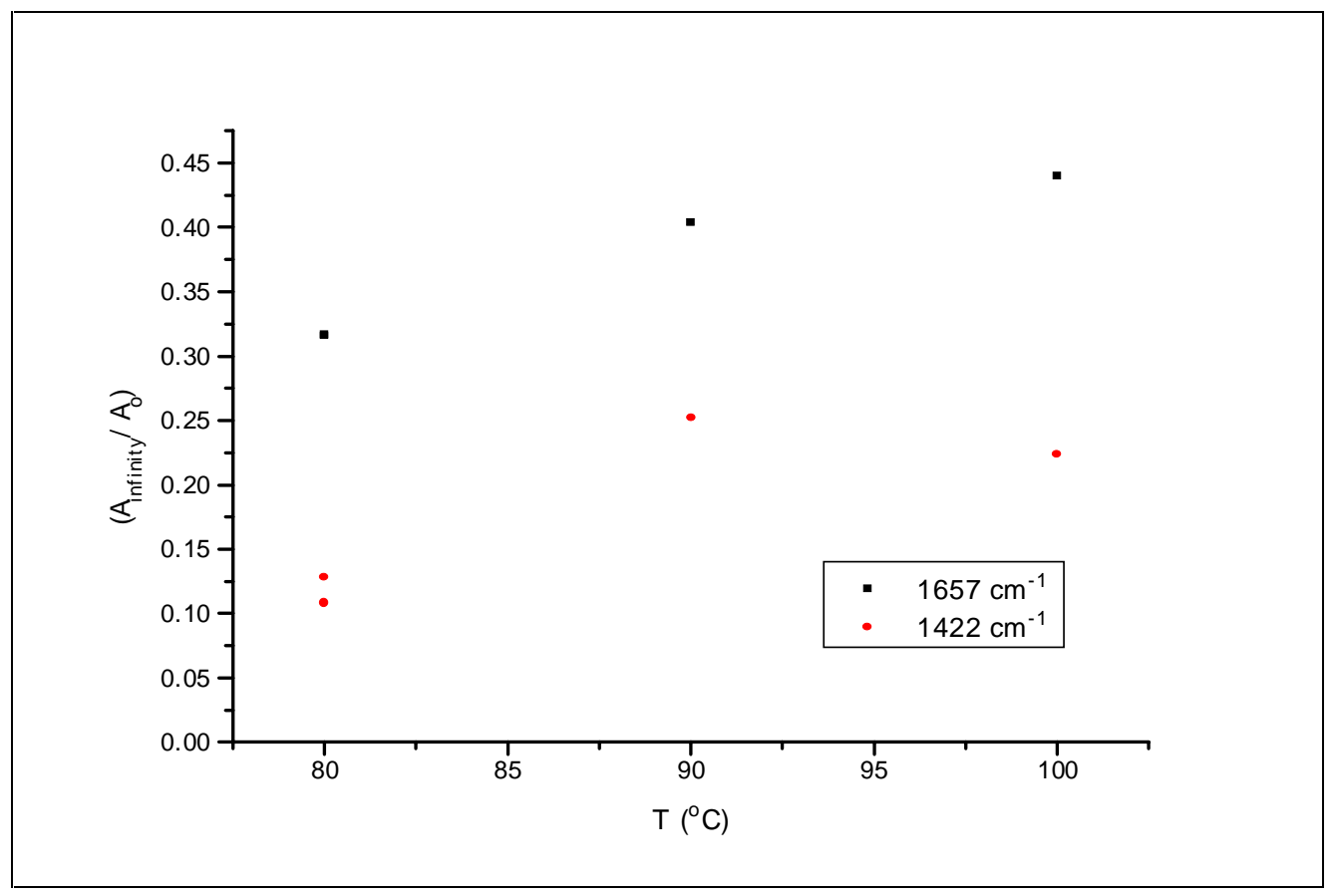

Figure 4.29: $\left(\mathrm{A}_{\propto} / \mathrm{A}_{\mathrm{o}}\right)_{\mathrm{PVP}}$ versus temperature

From the experimental values of $\mathrm{A}_{\propto}$ and $\mathrm{A}_{\mathrm{o}}$, it is theoretically possible to obtain the values of $\mathrm{a}$ and $\mathrm{b}$ from the equation (2.4.q). As a reminder, " $\mathrm{b}$ " represent the thickness of the PVP, whereas "a" is the total thickness of the interdiffusion system, the thickness of the VE being therefore $(a-b)$.

Since $(\mathrm{a}>>\mathrm{dp}), \exp \left(\frac{-2 \mathrm{a}}{\mathrm{dp}}\right) \approx 0$, and thus the equation (2.4.q) reduces to:

$$
\left(\frac{\mathrm{A}_{\infty}}{\mathrm{A}_{\mathrm{o}}}\right) \mathrm{PVP}=\left(\frac{\mathrm{b}}{\mathrm{a}}\right) * \frac{1}{\left[1-\exp \left(\frac{-2 \mathrm{~b}}{\mathrm{dp}}\right)\right]}
$$


For a given temperature, we get:

$$
\frac{\left(\frac{A_{\infty}}{A_{o}}\right)^{1}}{\left(\frac{A_{\infty}}{A_{o}}\right)^{2}}=\frac{\left[1-\exp \left(\frac{-2 b}{d_{2}}\right)\right]}{\left[1-\exp \left(\frac{-2 b}{d p_{1}}\right)\right]}
$$

where the subscripts 1 and 2 stand for a given characteristic band of the PVP

The equation (4.3.b) can be solved for b. Unfortunately, the attempts were unsuccessful. The software Mathematica was unable to solve numerically (4.3.b) in a reasonable time. It may have had origin in either the nonexistence or the multiplicity of solutions.

Knowing $\mathrm{dp}_{1}$ and $\mathrm{dp}_{2}$, it is also possible to guess a value for $\mathrm{b}$ and to compare the experimental ratio $\left[\left(\mathrm{A}_{\propto} / \mathrm{A}_{\circ}\right)_{1} /\left(\mathrm{A}_{\propto} / \mathrm{A}_{\circ}\right)_{2}\right]$, to the one obtained by (4.3.b) with this given value of $b$. At $T=100^{\circ} \mathrm{C}$ for instance, the experimental ratio $\left[\left(A_{\propto} / A_{\circ}\right)_{1} /\left(A_{\propto} / A_{\circ}\right)_{2}\right]$ was equal to 1.96635 , whereas the calculated ratio was about 1 . Table 4.12 summarizes some of the results obtained with different values of $b$. This data demonstrates that this ratio method is not very sensitive to changes in $b$.

Table 4.12: Theoretical ratio for different values of $b$

\begin{tabular}{|r|c||}
\hline $\mathrm{b}(\mathrm{in} \mu \mathrm{m})$ & \multicolumn{1}{c|}{ ratio } \\
\hline 1 & 0.68078 \\
\hline 3 & 0.98338 \\
\hline 10 & 0.99999 \\
\hline 80 & 1 \\
\hline
\end{tabular}




\subsubsection{2 - Theoretical Assumptions}

The diffusion model was formulated with some limiting assumptions.

The penetration depth, dp, was assumed to remain constant throughout the experiment, in other words the refractive index was considered as constant. However, the value of the refractive index of the PVP, $\mathrm{n}_{2}$, may have changed as the composition of the system changed with time. The index of refraction of the VE was unknown. Fortunately, the refractive indices of most organic compounds are very similar. The penetration depth could then be considered to be independent of the sample, all other factors being equal. Furthermore, by looking at equation (2.3.c), it is obvious that dp is not a strong function of $\mathrm{n}_{2}$. As a conclusion, the assumption of a constant penetration depth seems reasonable.

In order to get the expression of the concentration profile (equation (2.4.o)), the two phases were assumed to be completely miscible. Indeed, it has been shown by Dr. Hideko Oyama [144] that the PVP and the VE are totally miscible, independent of temperature. Equation (2.4.o) was also derived assuming no volume change upon mixing. This is probably wrong in the case of the strong (PVP/VE) interactions. The Fickian model does not consider the chemical interactions (Section 4.2) taking place in the system.

Both $\mathrm{D}_{(\mathrm{PVP})}$ and $\mathrm{D}_{(\mathrm{VE})}$ were obtained from equation (2.4.s). But only the diffusion coefficients obtained from study of the two peaks of the PVP seem to lead to accurate results, as pointed out in Subsection 4.1.5. One more assumption has been made in the derivation of the equation relating $\mathrm{D}_{(\mathrm{VE})}$ to the absorbance. This additional relation, expressed mathematically by equation (2.4.n), assumes that the reduction in the concentration profile of the PVP is due exclusively to the intrusion of the VE. This assumption is wrong when the volume does not remain constant. Furthermore, the mathematical model does not consider any difference in sizes between the two components of the system. 


\subsection{2 - HOW TO IMPROVE THE DIFFUSION MODEL?}

The ideal Fickian model has not been fully successful in modeling the experimental

data points. At $100^{\circ} \mathrm{C}$ for instance, this empirical model did not fit the data, especially in the early part of the diffusion. This is demonstrated in Figures 4.30, 4.31, 4.32 and 4.33, which show the curvefits of four peaks at $\mathrm{T}=100^{\circ} \mathrm{C}$.

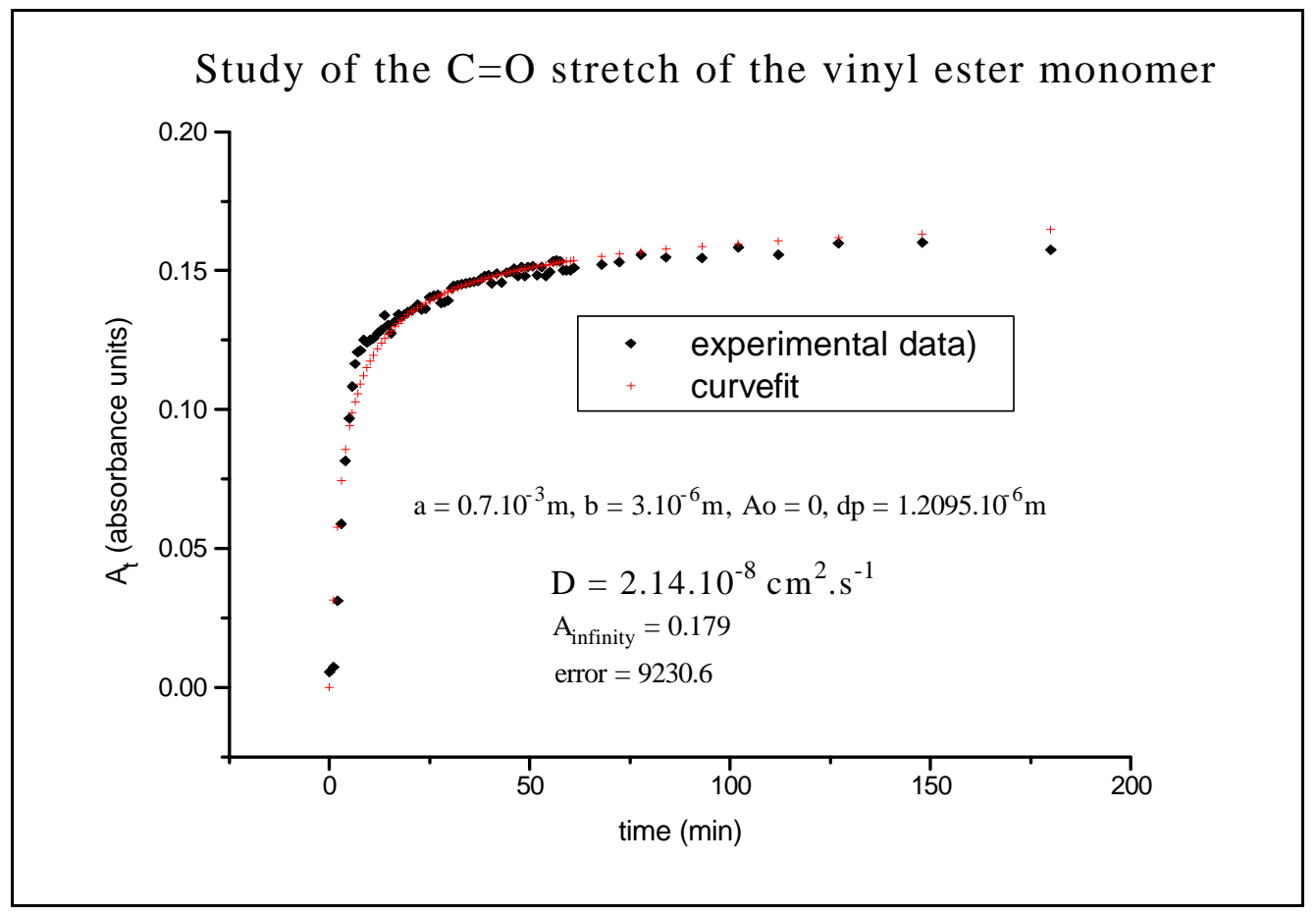

Figure 4.30: Curvefit of the peak at $1717 \mathrm{~cm}^{-1}$ at $\mathrm{T}=100^{\circ} \mathrm{C}$ 


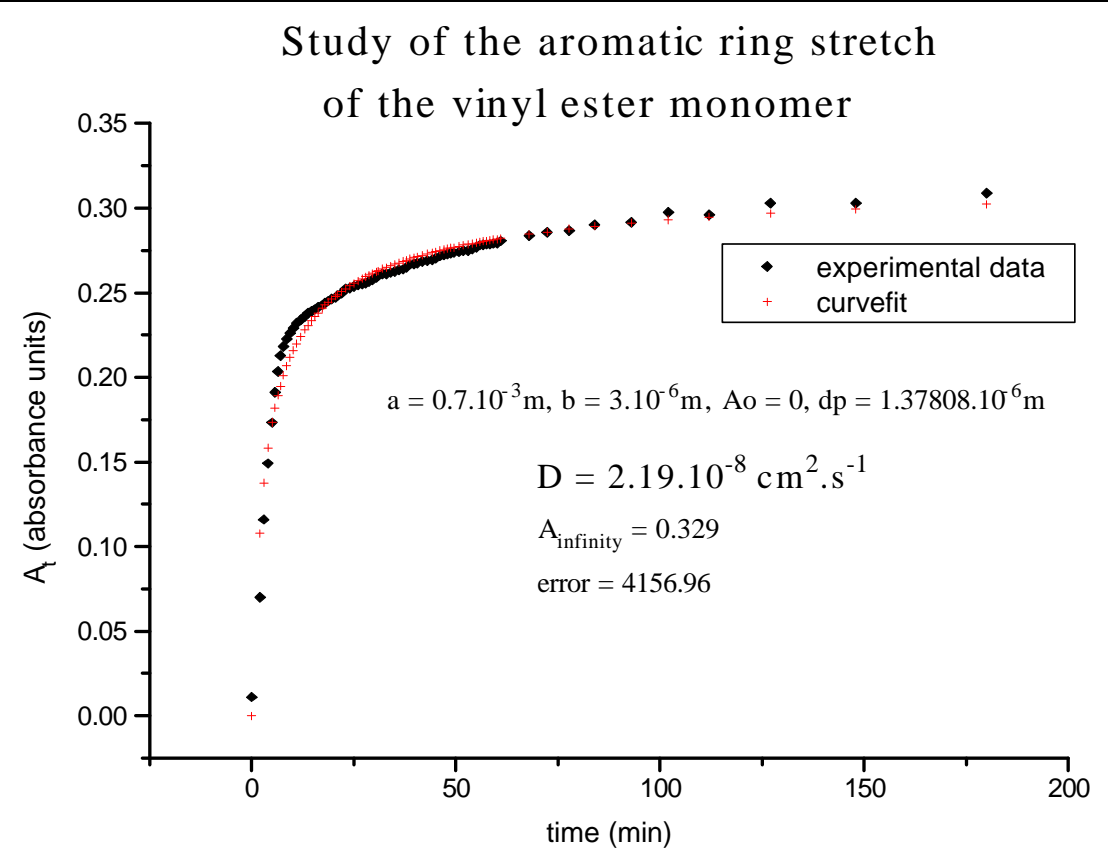

Figure 4.31: Curvefit of the peak at $1507 \mathrm{~cm}^{-1}$ at $\mathrm{T}=100^{\circ} \mathrm{C}$

Study of the $\mathrm{C}=\mathrm{O}$ and $\mathrm{N}-\mathrm{C}$ stretching vibrations of the PVP

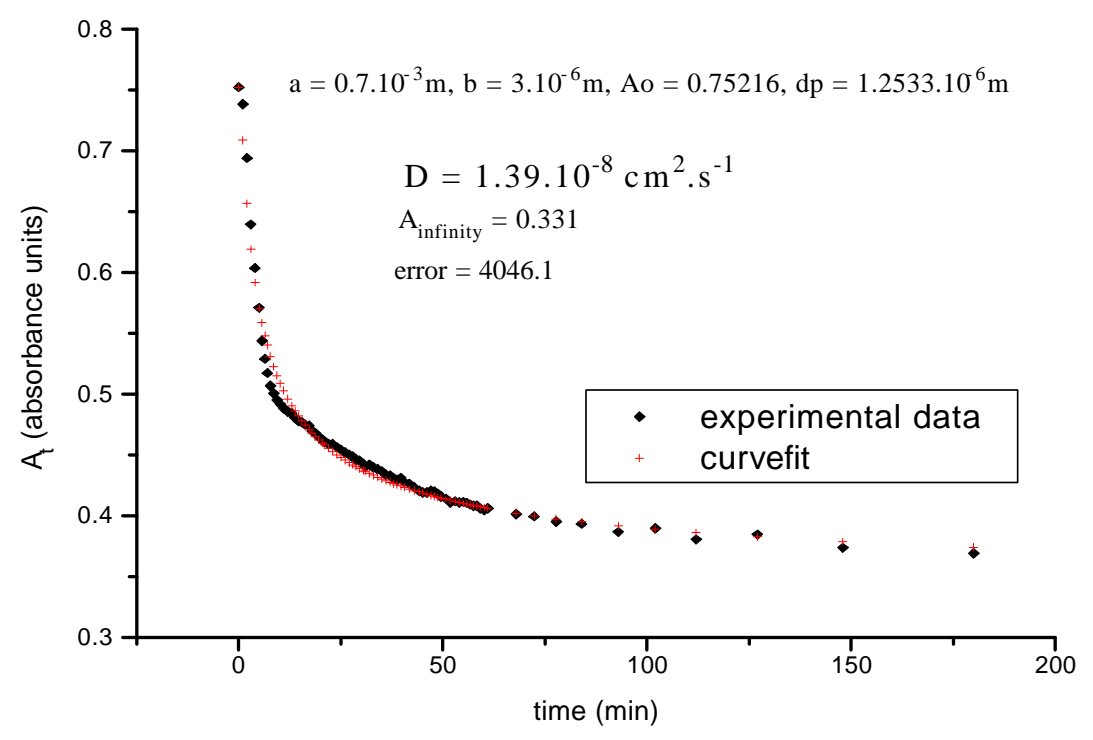

Figure 4.32: Curvefit of the peak at $1657 \mathrm{~cm}^{-1}$ at $\mathrm{T}=100^{\circ} \mathrm{C}$ 


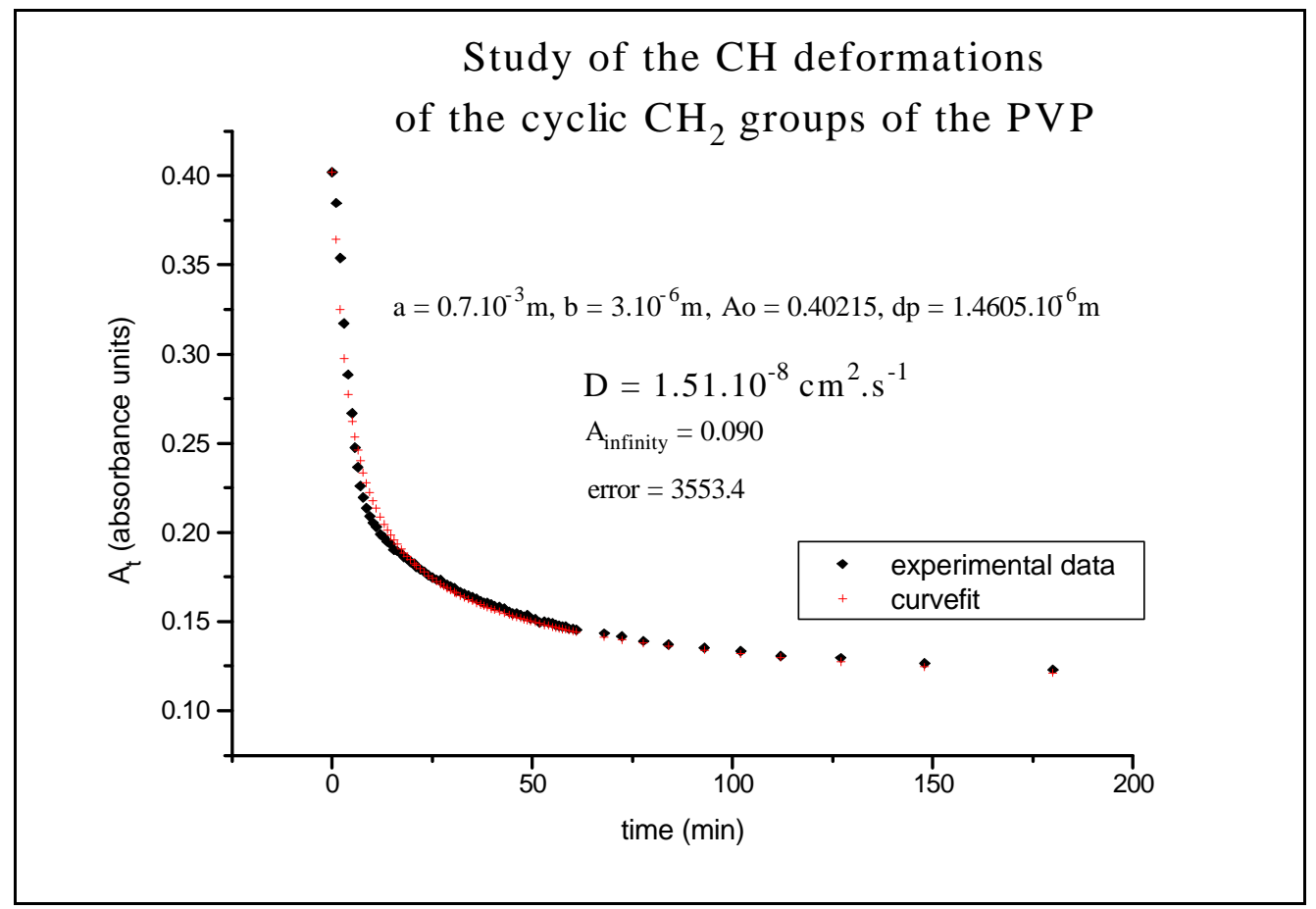

Figure 4.33: Curvefit of the peak at $1422 \mathrm{~cm}^{-1}$ at $\mathrm{T}=100^{\circ} \mathrm{C}$

Although some errors can be expected in fitting the data, it is unlikely that such large deviations between the experimental data and the curvefit would be observed entirely because of experimental errors. The analysis contains several limiting assumptions, such as the fact that the diffusion coefficients are independent on concentration. However, for a binary polymer mixture, the mutual diffusion coefficient is dependent on composition, temperature, compatibility, interactions, molecular weight, solubility, molecular weight distribution, chain orientation, size and shape, molecular structure, etc. Furthermore, the mutual diffusion coefficients of the two components are different. The simple Fickian model used as a first approximation in this study does not seem to be appropriate for the (PVP/VE) system. The diffusion process itself is probably more complex.

More suitable models have to be presented to accurately fit the data and therefore obtain a better agreement between experimental results and theoretical approach. A number of mechanisms can be invoked. These could include simultaneous Fickian models, 
plasticization, swelling, clustering, and a concentration dependent diffusion coefficient. The effect of these factors on the diffusion coefficient will be discussed in the following subsections.

\subsubsection{1 - Simultaneous Fickian Model}

An inspection of Figures 4.30 and 4.31 shows that at short interdiffusion times, the measured absorbance is lower than the one predicted by the Fickian model, while at longer times, the absorbance is higher. Consequently, a two stage-diffusion process may be better in modelling this data. For example, two simultaneous Fickian processes, assuming a constant diffusion coefficient, were employed for the (erucamide/isotactic polypropylene) system above $\mathrm{T}_{\mathrm{g}}$ by Quijada-Garrido et al. [147]. Furthremore, Dr. Hideko Oyama [144] showed using electron microprobe analysis (EMP), that for the (PVP/VER) bilayer films, the concentration profile could be divided into two parts. Each part was fit by a different constant diffusion coefficient assuming Fick's law.

\section{$\underline{4.3 .2 .2 ~-~ P l a s t i c i z a t i o n ~}$}

It was observed that at the end of the diffusion experiments, the PVP had changed from a rigid solid to a white soft gel. Actually, the VE has acted as a plasticizer of exceptionally high molecular weight $[157,158]$. Briefly, plasticizers are low molecular weight compounds added to soften a polymer by lowering its glass transition temperature and its rubbery plateau modulus, as well as its hardness, stiffness, and tensile strength [157, $159,160]$.

The Fox equation [134] gives the expression of the glass transition temperature, $\mathrm{T}_{\mathrm{g}}$, assuming that for each component, the product of the heat capacity at constant pressure by the $\mathrm{T}_{\mathrm{g}}$ remains constant. The Fox equation is defined below: 


$$
\left(\frac{1}{T_{g}}\right)=\left(\frac{M_{1}}{T_{g 1}}\right)+\left(\frac{M_{2}}{T_{g 2}}\right)
$$

where $M_{i}$ is the mass fraction of polymer $i$

The change in the glass transition temperature, as a function of the mass fraction of VE, was determined by Differential Scanning Calorimetry (DSC). DSC measurements were conducted using a Perkin-Elmer differential calorimeter. Solutions of PVP and VE in methanol were mixed together to achieve different compositions and dried under vacuum to evaporate all the solvent. The sample was held for $3 \mathrm{~min}$ at $-80^{\circ} \mathrm{C}$, heated from $-80^{\circ} \mathrm{C}$ to $70^{\circ} \mathrm{C}$ at a heating rate of $10^{\circ} \mathrm{C} / \mathrm{min}$, held for $3 \mathrm{~min}$ at $70^{\circ} \mathrm{C}$, cooled from $70^{\circ} \mathrm{C}$ to $-80^{\circ} \mathrm{C}$ at $100^{\circ} \mathrm{C} / \mathrm{min}$, held for $3 \mathrm{~min}$ at $-80^{\circ} \mathrm{C}$, and heated again from $-80^{\circ} \mathrm{C}$ to $70^{\circ} \mathrm{C}$ at $10^{\circ} \mathrm{C} / \mathrm{min}$.

$\mathrm{T}_{\mathrm{g}}$ 's of the blends were obtained from the second heating and plotted as a function of the mass fraction of the VE, as shown in Figure 4.34. A single $\mathrm{T}_{\mathrm{g}}$ was observed for the blends, confirming the miscibility of PVP and VE. The $\mathrm{T}_{\mathrm{g}}$ 's of the pure PVP and the pure VE were determined to be $\mathrm{T}_{\mathrm{g}(\mathrm{PVP})}=178^{\circ} \mathrm{C}$ and $\mathrm{T}_{\mathrm{g}(\mathrm{VE})}=9^{\circ} \mathrm{C}$, respectively. Theoretical values of $T_{g}$ were obtained by the Fox equation (4.3.c). The change in the $T_{g}$ with composition of (PVP/VE) blends followed the Fox equation over the range of investigation.

The change in the $\mathrm{T}_{\mathrm{g}}$ over the entire range of the mass fraction of $\mathrm{VE}$, is shown in Figure 4.35. This Figure shows the effects of the intrusion of the VE into the PVP. As the VE penetrates into the PVP, there is a decrease in $T_{g}$ with plasticization. The $T_{g}$ of the PVP was constantly evolving during the experiment. The polymer softened while the amount of VE increased. Since the polymer could imbibe a penetrant only to a given concentration, the polymer changed drastically from glassy to rubbery at a critical concentration of small molecules $[157,161]$. At $\mathrm{T}=100^{\circ} \mathrm{C}$ for instance, this change was observed for a mass fraction of VE equal to 0.35 . 


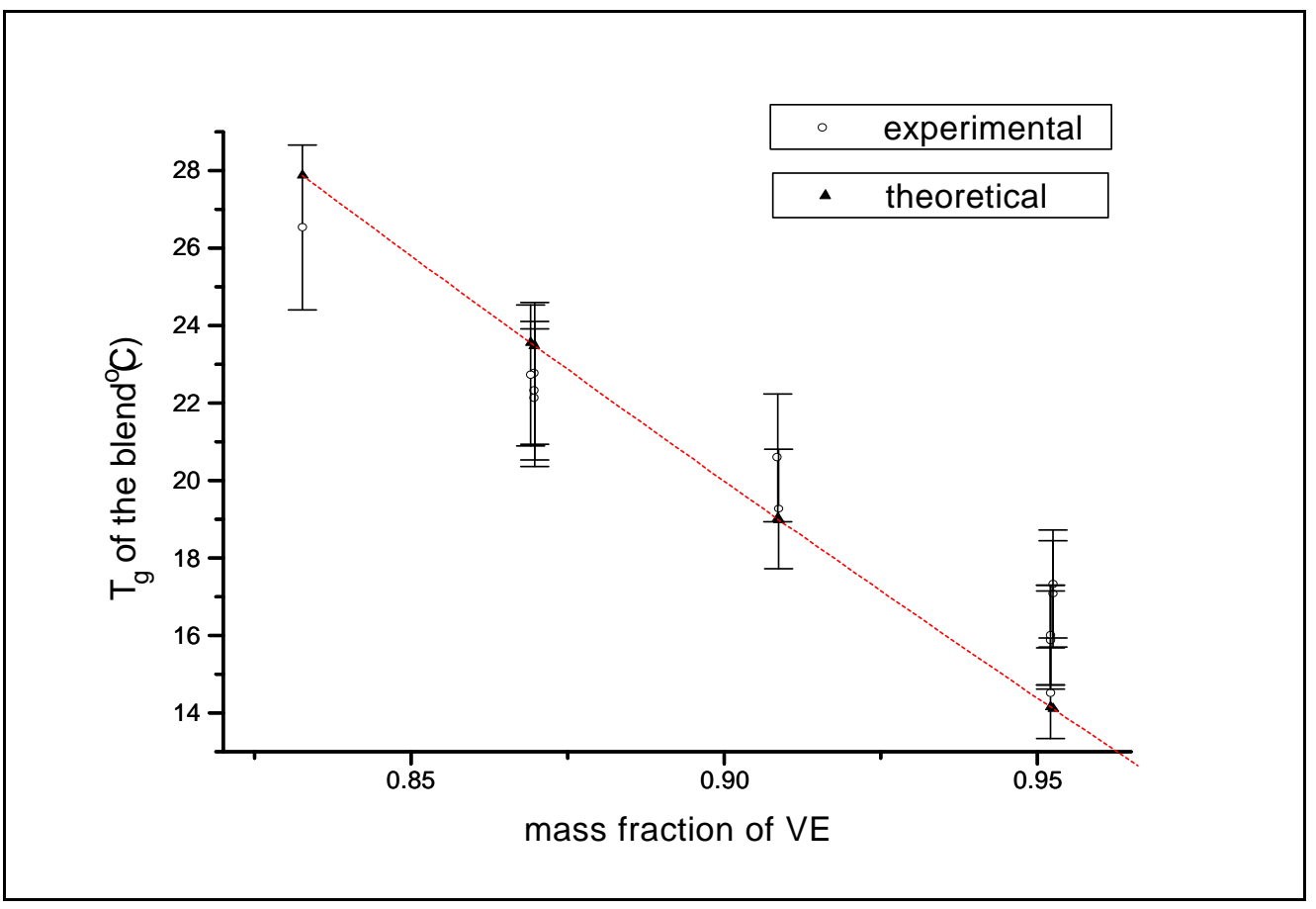

Figure 4.34: Experimental and theoretical $\mathrm{T}_{\mathrm{g}}\left({ }^{\circ} \mathrm{C}\right)$ versus the mass fraction of VE

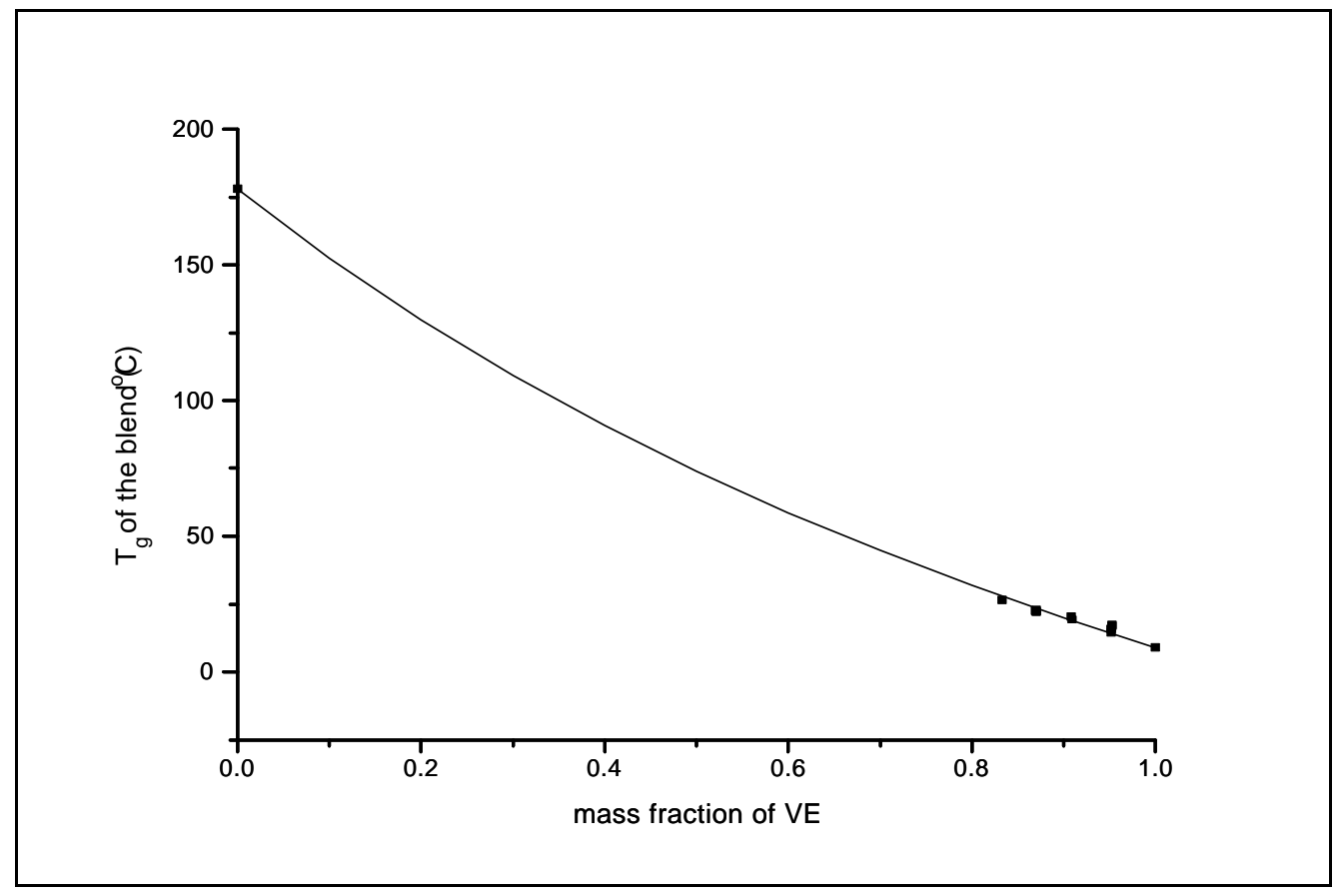

Figure 4.35: $\mathrm{T}_{\mathrm{g}}$ versus mass fraction of VE 
The change in the $T_{g}$ as a function of the interdiffusion time could also be determined from the results obtained by FTIR-ATR spectroscopy. Once the concentrations of the PVP and the VE as a function of time are known, it is possible to calculate the mole fraction and the mass fraction of the $\mathrm{VE}$, and then to deduct the $\mathrm{T}_{\mathrm{g}}$ from equation (4.3.c).

The mean concentration of the PVP within the penetration depth at a given time, $<\mathrm{C}(\mathrm{t})>_{\text {PVP }}$, can be calculated as follows:

$$
<\mathrm{C}(\mathrm{t})\rangle_{\mathrm{PVP}}=\left[\frac{\int_{0}^{\infty} \operatorname{CPVP}(\mathrm{z}, \mathrm{t}) \cdot \exp \left(\frac{-2 \mathrm{z}}{\mathrm{dp}}\right) \cdot \alpha \cdot \operatorname{S} \cdot \mathrm{dz}}{\int_{0}^{\infty} \exp \left(\frac{-2 \mathrm{z}}{\mathrm{dp}}\right) \cdot \alpha \cdot \mathrm{S} \cdot \mathrm{dz}}\right]
$$

where $\langle\mathrm{C}(\mathrm{t})\rangle_{\mathrm{PVP}}$ is the mean concentration of the PVP

$\mathrm{C}_{\mathrm{PVP}}(\mathrm{z}, \mathrm{t})$ is the concentration profile of the PVP

$\mathrm{z}$ is the distance from the ATR crystal

$\mathrm{dp}$ is the penetration depth

$\alpha$ is the characteristic absorptivity

$\mathrm{S}$ is the cross-sectional area

The numerator corresponds to the expression of the absorbance of the PVP, $A_{P V P}(t)$, for a given time, as defined by the equation (2.4.1).

Then

$$
<\mathrm{C}(\mathrm{t})\rangle_{\mathrm{PVP}}=\left[\frac{\operatorname{APVP}(\mathrm{t})}{-\alpha_{\text {PVP.SPVP. }}\left(\frac{\mathrm{dp}}{2}\right) \cdot\left[\exp \left(\frac{-2 \mathrm{z}}{\mathrm{dp}}\right)\right]_{0}^{\infty}}\right]
$$

assuming that $\mathrm{dp}, \alpha_{\mathrm{PVP}}$, and $\mathrm{S}_{\mathrm{PVP}}$ remain constant 


$$
\text { Finally } \quad<\mathrm{C}(\mathrm{t})>_{\mathrm{PVP}}=\left[\frac{2 \cdot \operatorname{APVP}(\mathrm{t})}{\alpha_{\mathrm{PVP} .} \operatorname{SPVP}_{\mathrm{PVPPVP}} \cdot \mathrm{dp}_{\mathrm{P}}}\right]
$$

Similarly, the cumulative concentration of the $\mathrm{VE},\langle\mathrm{C}(\mathrm{t})\rangle_{\mathrm{VE}}$, can be defined as:

$$
<\mathrm{C}(\mathrm{t})\rangle_{\mathrm{VE}}=\left[\frac{2 \cdot \operatorname{Ave}(\mathrm{t})}{\alpha_{\text {VE. }} \operatorname{SVE}_{\text {Ve. }} \mathrm{dpve}_{\mathrm{VE}}}\right]
$$

The mole fraction of the VE at a particular interdiffusion time, molf $\mathrm{VE}_{\mathrm{E}}(\mathrm{t})$, can then be calculated easily from (4.3.d) and (4.3.e). Its expression is:

$$
\begin{aligned}
& \operatorname{molfve}(t)=\left[\frac{<\mathrm{C}(\mathrm{t})\rangle \mathrm{VE}}{\{\langle\mathrm{C}(\mathrm{t})\rangle \mathrm{VE}\}+\{<\mathrm{C}(\mathrm{t})>\mathrm{PVP}\}}\right] \\
& =\left[\frac{\operatorname{AvE}(t)}{\{\operatorname{AvE}(t)\}+\left\{\left(\frac{d p v E}{d p P V P}\right) \cdot\left(\frac{\alpha_{V E}}{\alpha_{P V P}}\right) \cdot\left(\frac{S_{V E}}{S_{P V P}}\right) \cdot \operatorname{APVP}(t)\right\}}\right]
\end{aligned}
$$

Since the cross-sectional area is the same, $\mathrm{S}_{\mathrm{VE}}=\mathrm{S}_{\mathrm{PVP}}$, (4.3.f) reduces to:

$$
\operatorname{mol} f_{V E}(t)=\left[\frac{\operatorname{Ave}(t)}{\{\operatorname{AvE}(t)\}+\left\{\left(\frac{d p v E}{d p_{P V P}}\right) \cdot\left(\frac{\alpha_{V E}}{\alpha_{P V P}}\right) \cdot \operatorname{APVP}(t)\right\}}\right]
$$

Let's apply equation (4.3.g) to the calculation of the mole fraction of the carbonyl groups in the PVP and in the VE. These groups have vibrational frequencies at $1664 \mathrm{~cm}^{-1}$ and $1717 \mathrm{~cm}^{-1}$, respectively, as reported in Table 4.3. As a first approximation, one can 
assume that $\alpha_{\mathrm{PVP}}=\alpha_{\mathrm{VE}}$. It is well-known that the absorptivity $\alpha$ changes with the wavenumber and the molecule [109]. However, when studying the same functional groups, one can assume that the absorptivities will not change that much [151].

The mole fraction of VE carbonyl groups, $\operatorname{molf}_{\mathrm{VE}(\mathrm{C}=\mathrm{O})}(\mathrm{t})$, can then be expressed by:

$$
\operatorname{molfvE}(C=0)(t)=\left[\frac{\operatorname{AvE}(C=0)(t)}{\operatorname{AvE}(C=0)(t)+\left(\frac{d p v E}{d p_{P V P}}\right) \cdot \operatorname{APVP}(C=0)(t)}\right]
$$

The results obtained at $80^{\circ} \mathrm{C}$ based on the carbonyl groups of the PVP and the VE are shown in Figure 4.36.

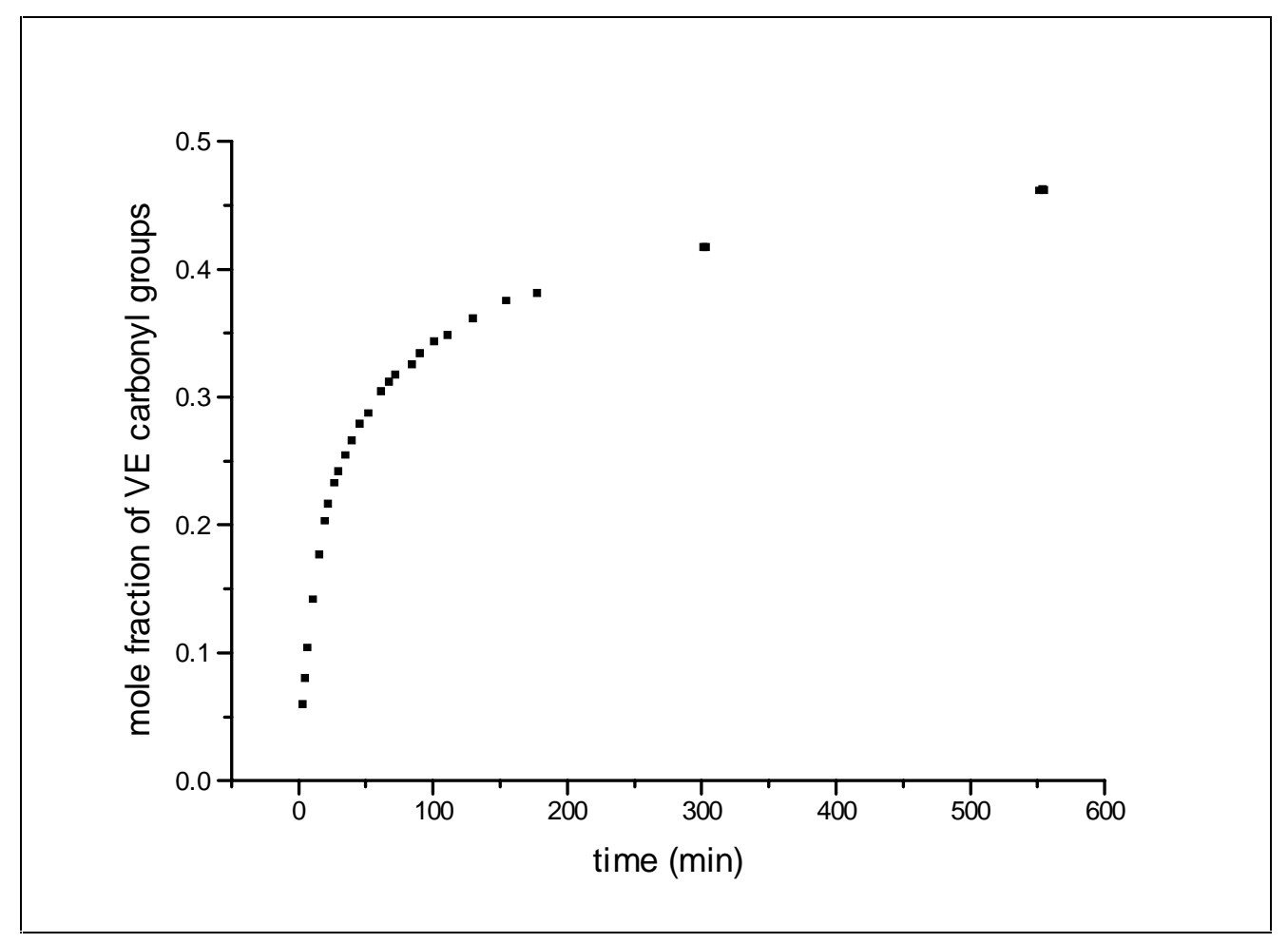

Figure 4.36: Mole fraction of VE carbonyl groups versus interdiffusion time at $\mathrm{T}=80^{\circ} \mathrm{C}$ 
In order to express the $T_{\mathrm{g}}$ as a function of the interdiffusion time, a relationship between the mole fraction of VE carbonyl groups and the mass fraction of VE has to be established.

The mole fraction of VE carbonyl groups can also be defined as:

$$
\left.\operatorname{molfVE}_{(\mathrm{C}}=\mathrm{O}\right)(\mathrm{t})=\left[\frac{\mathrm{n}_{(\mathrm{C}=O \text { in } \mathrm{VE})}}{\mathrm{n}_{(\mathrm{C}=O \text { in } V E)}+\mathrm{n}_{(\mathrm{C}=O \text { in } P V P)}}\right]
$$

where $\mathrm{n}$ stands for the number of moles

There are 9910 carbonyl groups in one mole of PVP and two carbonyl groups in one mole of VE (see Figures 3.1 and 3.2). The number of carbonyl groups in one mole of PVP is indeed equal to the number of repeat units in the polymer.

Then

$$
\operatorname{molfVE}(\mathrm{C}=0)(\mathrm{t})=\left[\frac{2 *\left(\frac{\mathrm{mVE}_{\mathrm{VE}}}{\mathrm{MVE}_{\mathrm{VE}}}\right)}{2 *\left(\frac{\mathrm{m}_{\mathrm{VE}}}{\mathrm{MVE}_{\mathrm{VE}}}\right)+9910 *\left(\frac{\mathrm{mPVP}}{\mathrm{M}_{\mathrm{PVP}}}\right)}\right]
$$

where $\mathrm{m}_{\mathrm{VE}}$ is the mass of the VE

$\mathrm{m}_{\mathrm{PVP}} \quad$ is the mass of the PVP

$\mathrm{M}_{\mathrm{VE}}$ is the molecular weight of the VE. It is equal to $690 \mathrm{~g} / \mathrm{mol}$

$\mathrm{M}_{\mathrm{PVP}}$ is the molecular weight of the PVP. It is equal to $1,100,000 \mathrm{~g} / \mathrm{mol}$

Finally

$$
\left.\operatorname{molfVE}_{(\mathrm{C}}=0\right)(\mathrm{t})=\left[\frac{\mathrm{mVE}}{\operatorname{mvE}+\left(\frac{9910}{2}\right) \cdot\left(\frac{\mathrm{MVE}_{\mathrm{VE}}}{\mathrm{MPVP}_{\mathrm{PV}}}\right) \cdot \mathrm{mPVP}}\right]
$$


The mass fraction of $\mathrm{VE}$, $\operatorname{mass}_{\mathrm{VE}}(\mathrm{t})$, is defined as follows:

$$
\operatorname{massfve}(t)=\left[\frac{\operatorname{mVE}}{\operatorname{mVE}+\operatorname{mPVP}}\right]
$$

Let's take $m_{V E}+m_{P V P}=100 \mathrm{~g}$. Then, from the value of the mass fraction of VE, one can calculate $\mathrm{m}_{\mathrm{VE}}$ and $\mathrm{m}_{\mathrm{PVP}}$ from equation (4.3.j), and finally calculate the mole fraction of VE carbonyl groups from (4.3.I). Figure 4.37 indicates the mass fraction of VE as a function of the mole fraction of VE carbonyl groups.

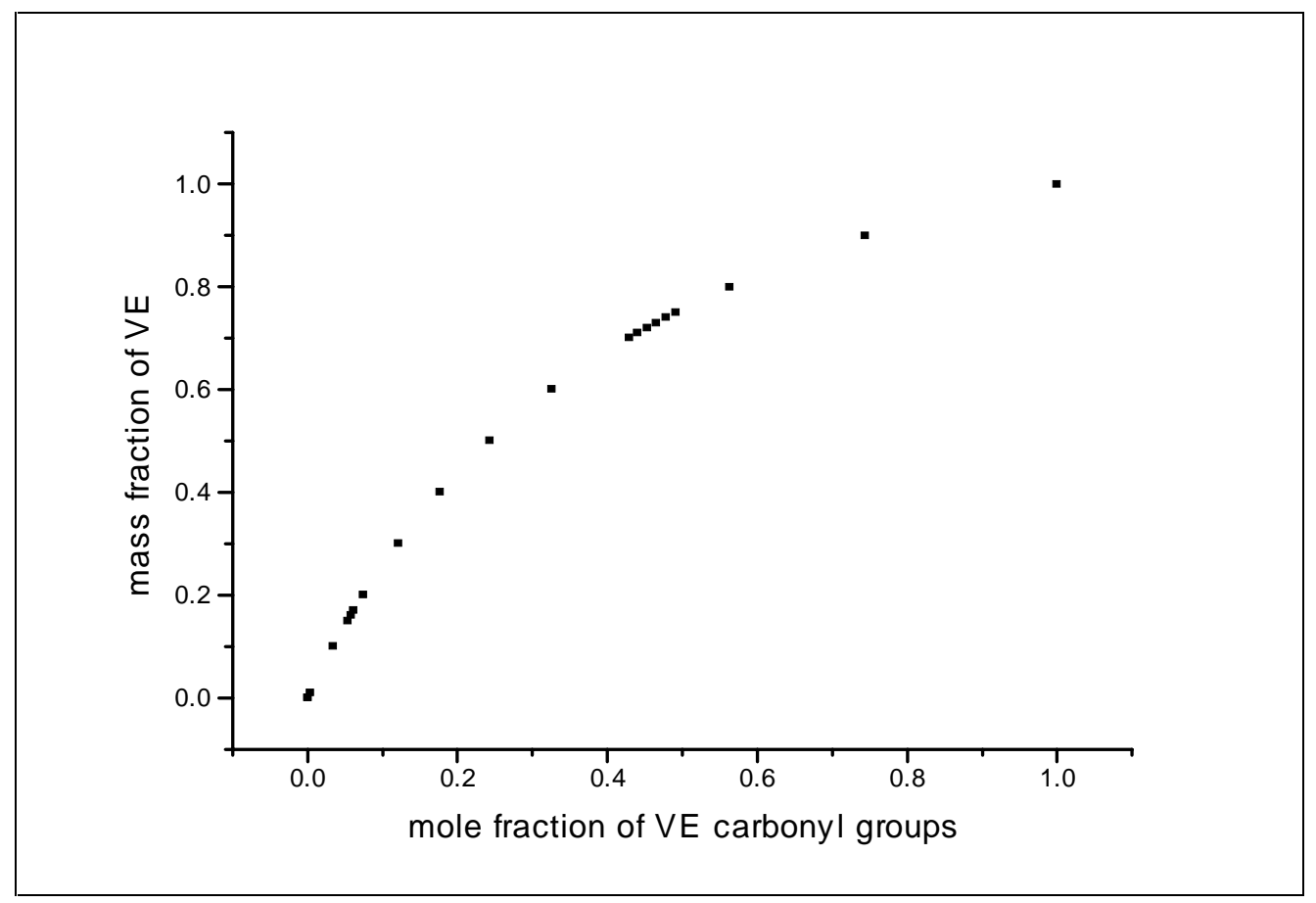

Figure 4.37: Mass fraction of VE versus mole fraction of VE carbonyl groups

The data points of Figure 4.37 were fit with a polynomial regression of order 4; the correlation coefficient, R, was equal to 0.99998 . This polynomial had the expression: 


$$
y=0.00212+2.94871 x-4.56824 x^{2}+4.11307 x^{3}-1.49605 x^{4}
$$

Using this polynomial, one is able to calculate the mass fraction of VE from the value of molf $_{\mathrm{VE}(\mathrm{C}=\mathrm{O})}(\mathrm{t})$. The data points of Figure 4.36 were converted to the mass fraction of VE as a function of time, as demonstrated in Figure 4.38.

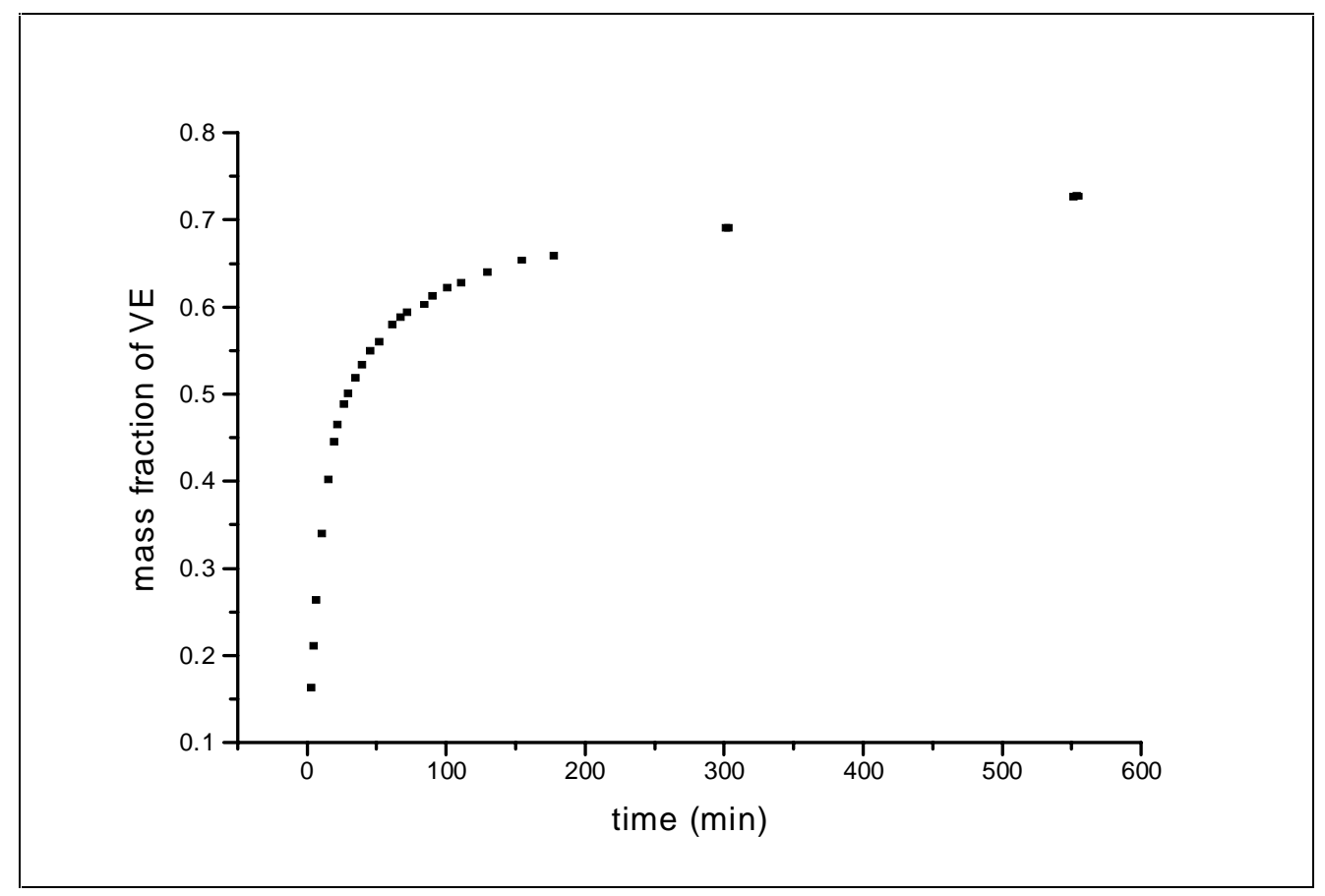

Figure 4.38: Mass fraction of VE versus interdiffusion time at $\mathrm{T}=80^{\circ} \mathrm{C}$

The relationship between the mass fraction of VE and the $\mathrm{T}_{\mathrm{g}}$ can be obtained from the Fox equation (4.3.c). The results obtained at $80^{\circ} \mathrm{C}$ for the evolution of the $T_{g}$ as a function of the interdiffusion time are shown in Figure 4.39. An interesting conclusion can be deducted from Figure 4.39. The polymer became rubbery after $22 \mathrm{~min}$ of diffusion at $\mathrm{T}=$ $80^{\circ} \mathrm{C}$. No obvious discontinuities were noticed before this particular time in Figures 4.10, $4.11,4.12$, and 4.13 . 


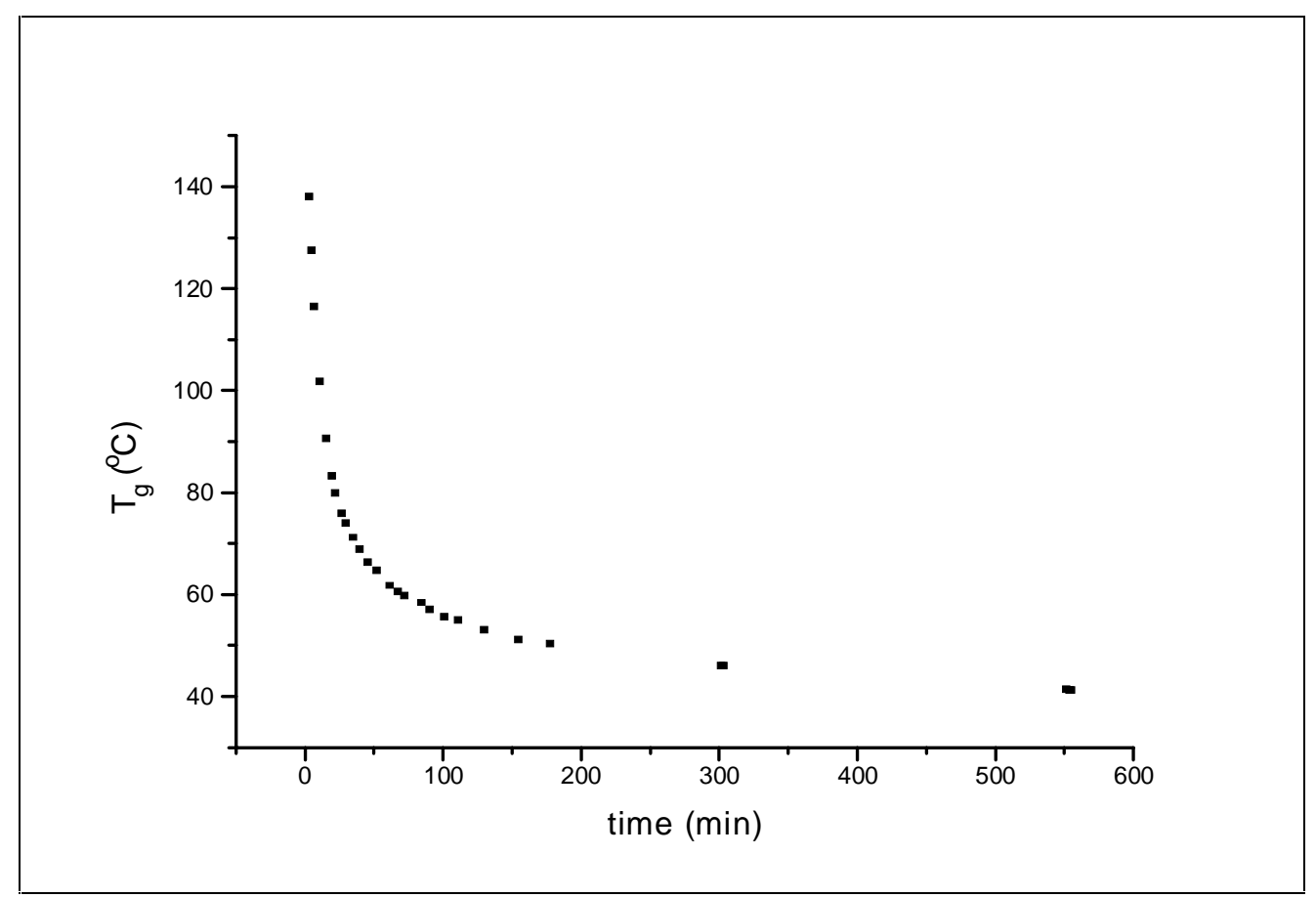

Figure 4.39: $\mathrm{T}_{\mathrm{g}}$ versus interdiffusion time at $\mathrm{T}=80^{\circ} \mathrm{C}$

The analysis was repeated for different temperatures. The evolution of the mass fraction of $\mathrm{VE}$ and the glass transition temperature with time at three different temperatures is reported in Figures 4.40 and 4.41, respectively. Figure 4.40 shows the effect of temperature on the mass fraction of VE. The higher the temperature, the faster the equilibrium is reached. As a consequence, the $T_{g}$ decreases more rapidly at higher temperature (see Figure 4.41). From Figure 4.41, one is able to determine the particular time, $\tau$, at which the temperature and the $\mathrm{T}_{\mathrm{g}}$ are identical. The results are summarized in Table 4.13. At $\tau$, the material changes from a glassy to a rubbery state. 


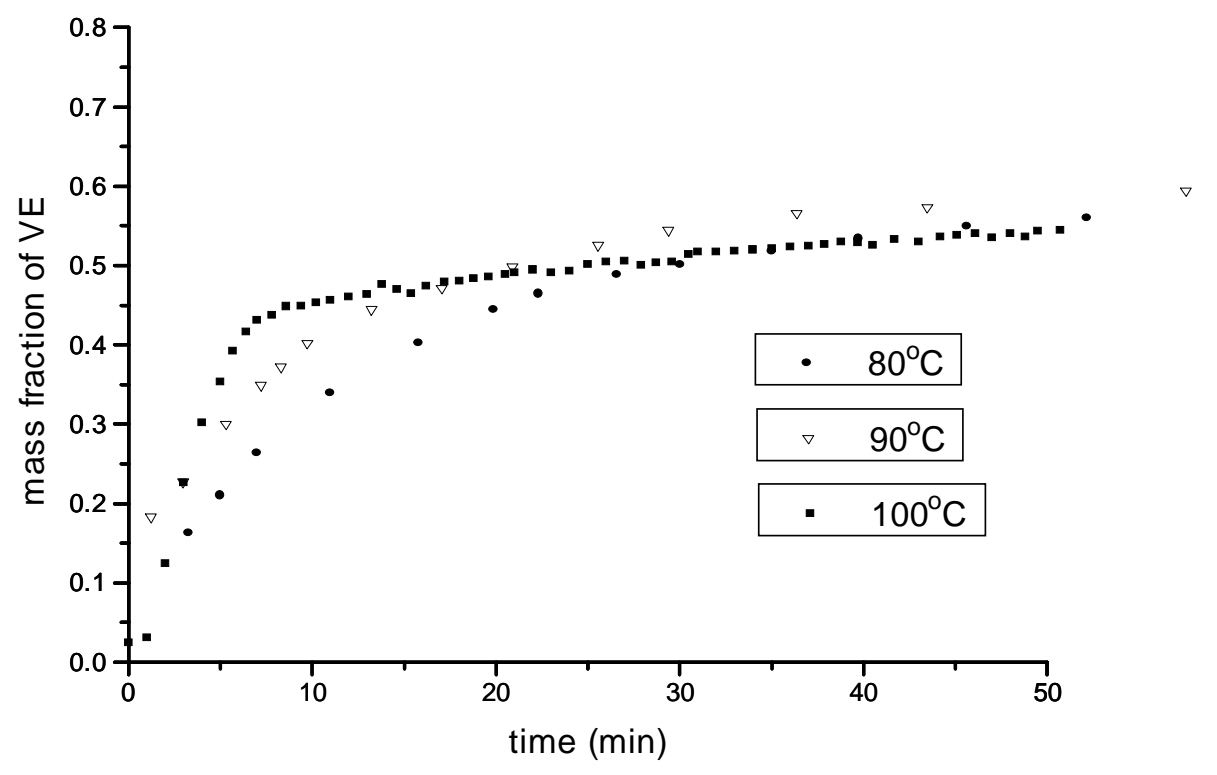

Figure 4.40: Mass fraction of VE versus time for different temperatures

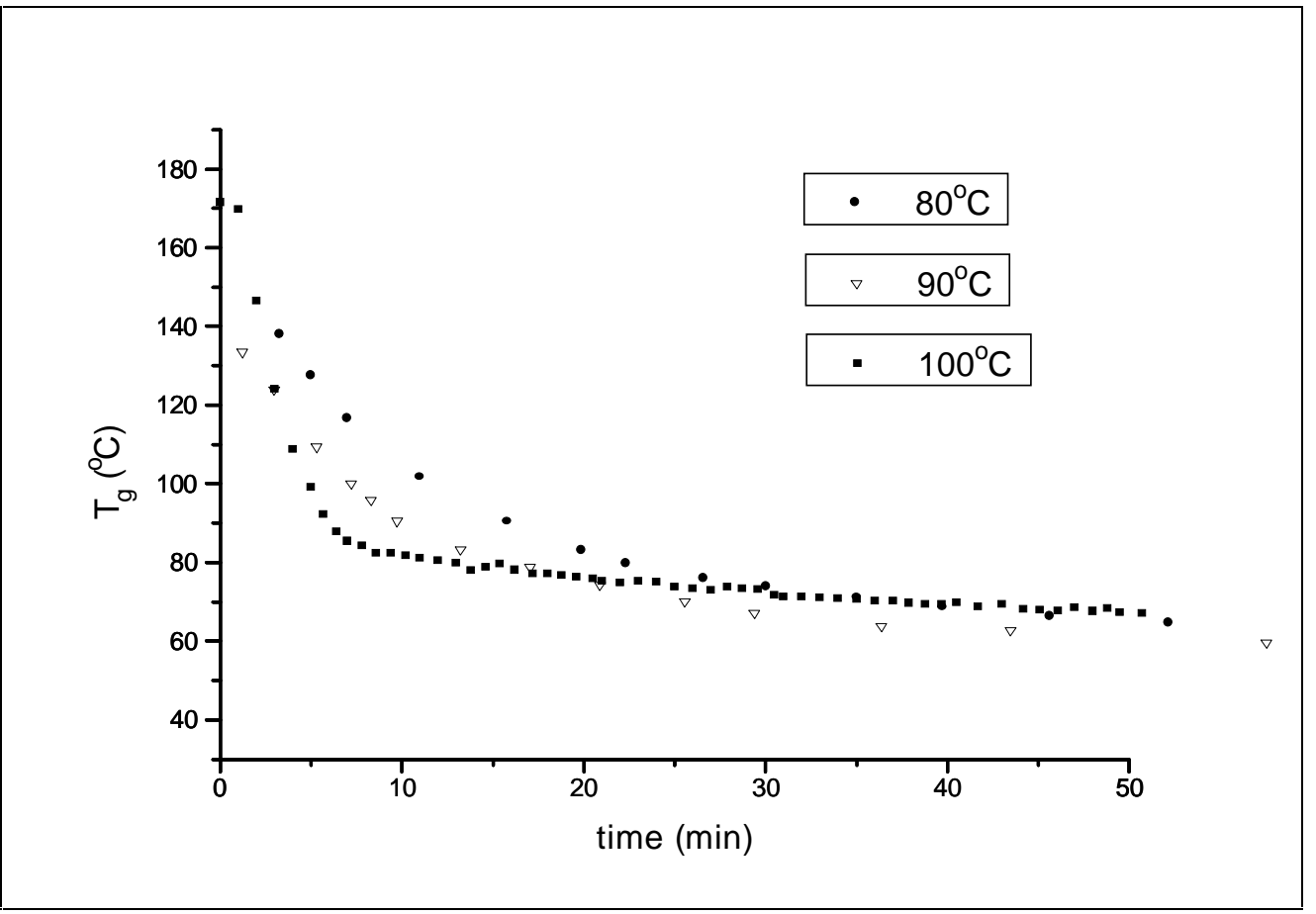

Figure 4.41: $\mathrm{T}_{\mathrm{g}}$ versus time for different temperatures 
Table 4.13: Interdiffusion time for which $\mathrm{T}=\mathrm{T}_{\mathrm{g}}$

\begin{tabular}{|r|r|}
\hline \hline temperature $\left({ }^{\circ} \mathrm{C}\right)$ & time $(\mathrm{min})$ \\
\hline 80 & 22 \\
\hline 90 & 10 \\
\hline 100 & 5 \\
\hline \hline
\end{tabular}

While monitoring the absorbance with time, $\tau$ occurs at approximately $\left(\mathrm{A}_{\alpha} / 2\right)$ for the $1717 \mathrm{~cm}^{-1}$ peak of the VE. Thus, the change from a glassy to a rubbery material will probably have a critical effect on the value of the diffusion coefficient. The curvefit at $100^{\circ} \mathrm{C}$, shown in Figure 4.30 , looks probably worse than the one at $80^{\circ} \mathrm{C}$, illustrated in Figure 4.10 , because the state of the material changes very rapidly at higher temperatures, and hence the value of the diffusion coefficient can evolve continuously. Actually, a distinct point was observed in the early part of the curvefit of Figure 4.30. At $t=\tau=5$ min at $T=$ $100^{\circ} \mathrm{C}$, the curvefit intercepts the experimental data points, as shown in Figure 4.42.

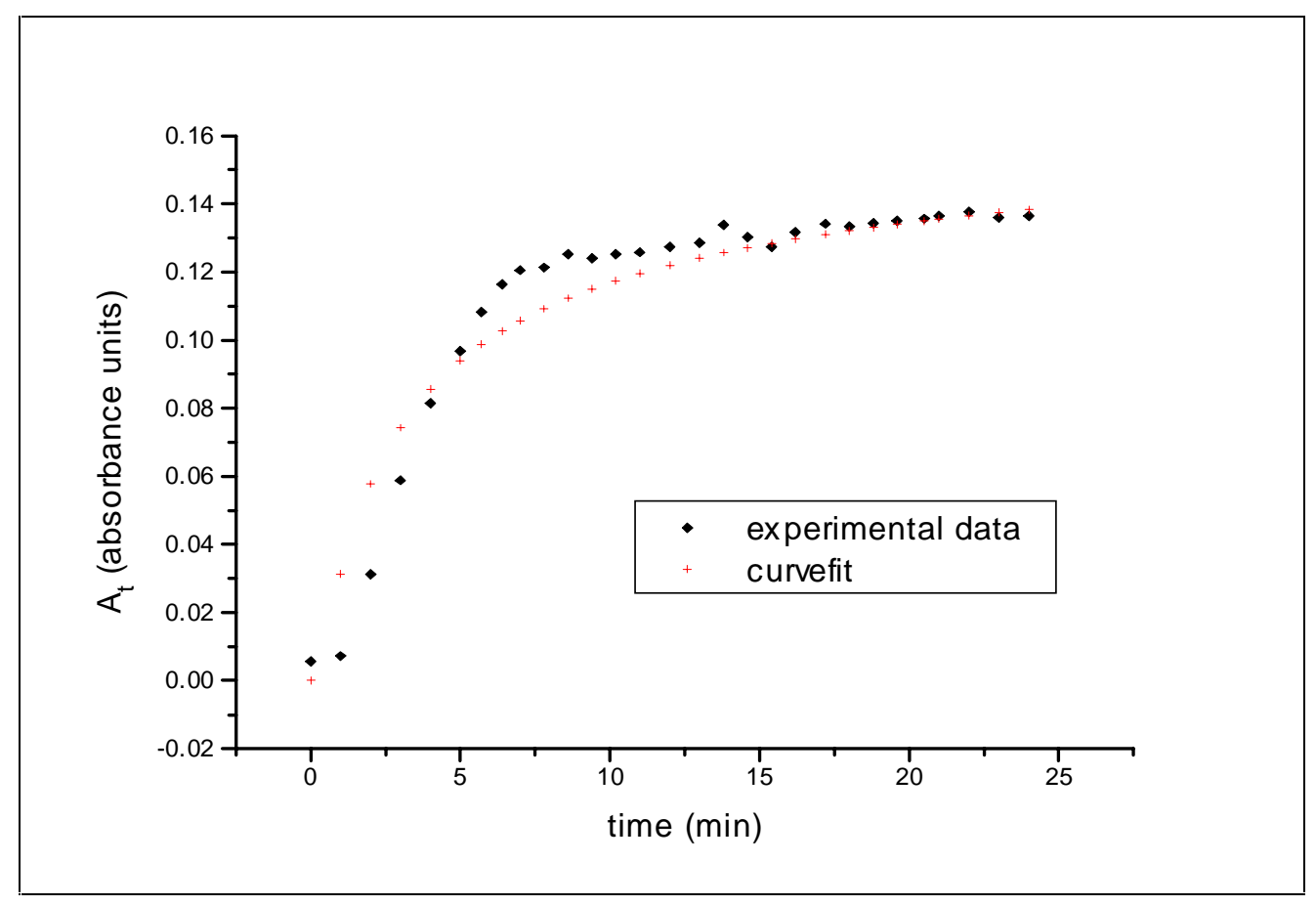

Figure 4.42: Zoom in the early part of Figure 4.30 
If the five data points recorded before $\tau$ are canceled for the $1717 \mathrm{~cm}^{-1}$ peak at $100^{\circ} \mathrm{C}$, the curvefit looks much better, as shown in Figure 4.43, than when the entire data set is used in the curvefit.

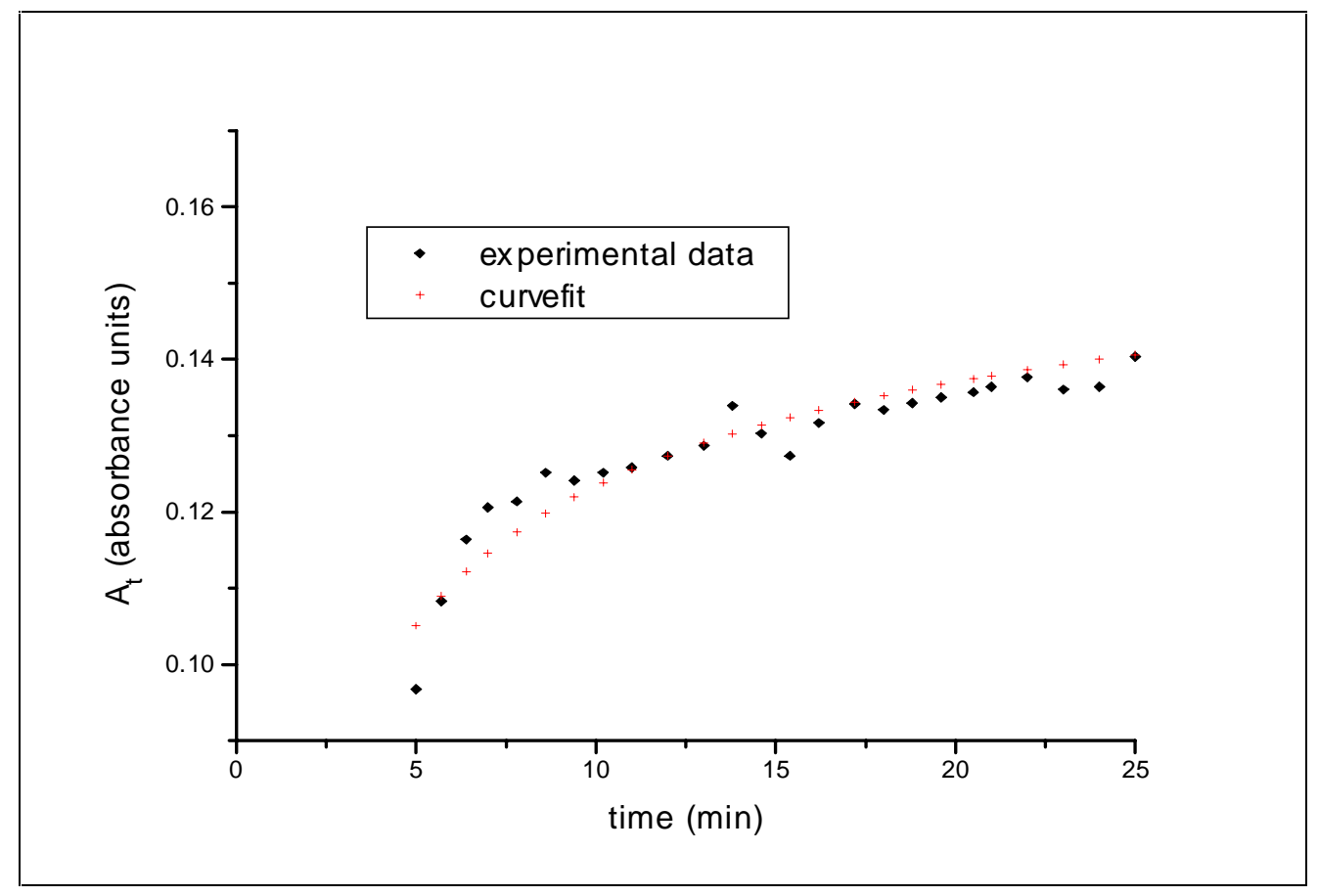

Figure 4.43: Curvefit of the $1717 \mathrm{~cm}^{-1}$ peak at $100^{\circ} \mathrm{C}$ cropping the first five data points

When curvefitting the truncated data set, the value of the diffusion coefficient increases from 2.14. $10^{-8} \mathrm{~cm}^{2} \cdot \mathrm{s}^{-1}$ to $3 \cdot 49 \cdot 10^{-8} \mathrm{~cm}^{2} \cdot \mathrm{s}^{-1}$. However, the error introduced with the data point at $\mathrm{t}=\tau$ is still high. If the data point obtained at $\mathrm{t}=\tau$ is removed, then the curvefit is even better, as shown in Figure 4.44. The value of the diffusion coefficient is then equal to 3.65. $10^{-8} \mathrm{~cm}^{2} \cdot \mathrm{s}^{-1}$. 


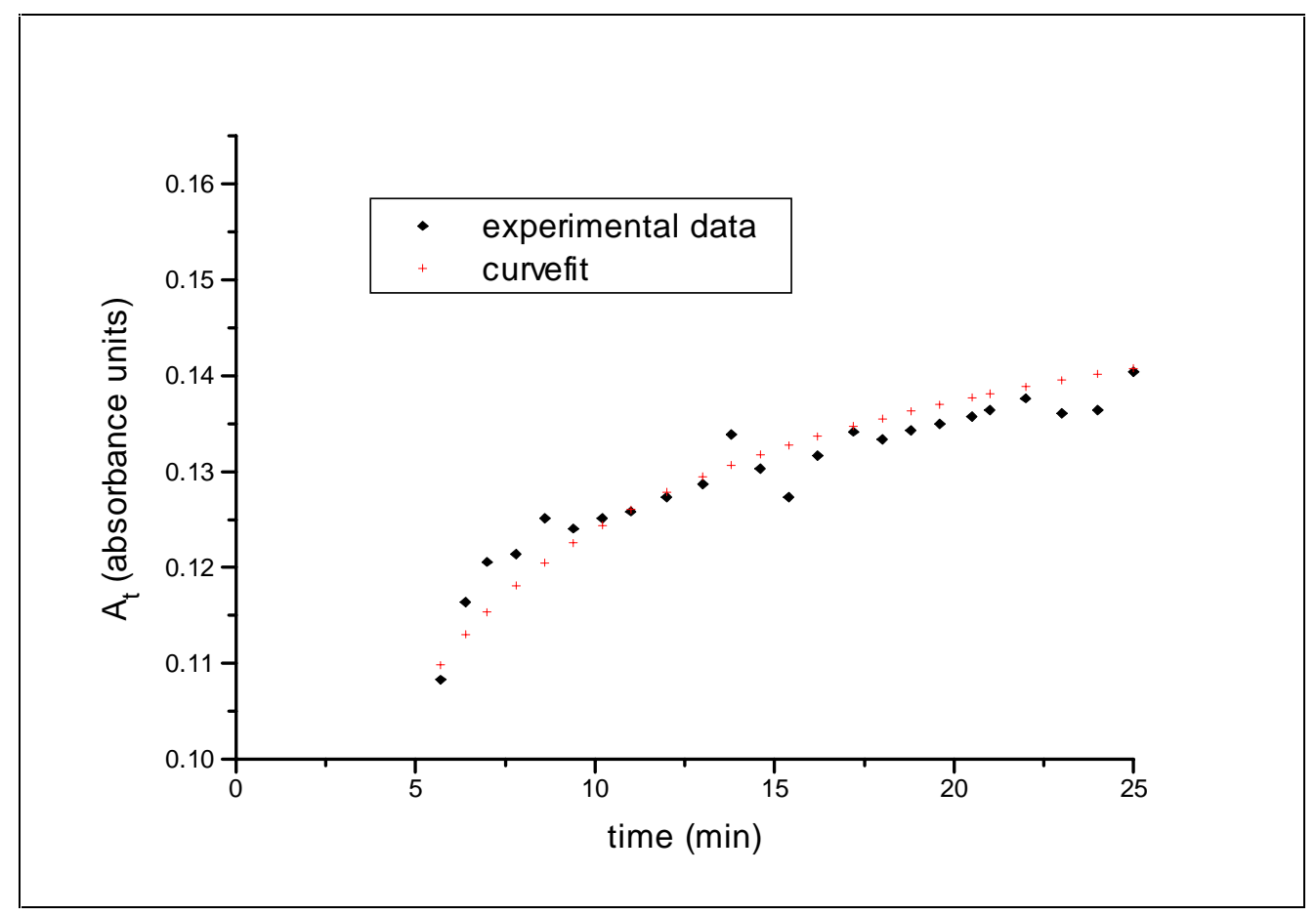

Figure 4.44: Curvefit of the $1717 \mathrm{~cm}^{-1}$ peak at $100^{\circ} \mathrm{C}$ cropping the first six data points

Mass transport is limited primarily by the $\mathrm{T}_{\mathrm{g}}$. Below the $\mathrm{T}_{\mathrm{g}}$, the polymer is hard, brittle, glassy, and only vibrational and short-range rotational motions are possible. Only the portions of chains containing a few monomers are able to move in microscopic regions. At the $\mathrm{T}_{\mathrm{g}}$, the polymer softens and becomes rubbery. Above the $\mathrm{T}_{\mathrm{g}}$, rapid molecular motions separate the chains from each other, because of the increase in free volume, and hence make reptation and diffusion easier. Molecular motion cannot take place without the presence of free volume, which increases with the incorporation of VE into the PVP, causing plasticization. The increase of free volume with temperature is dillustrated in Figure 4.45. $\mathrm{v}_{0, \mathrm{G}}$ and $\mathrm{v}_{0, \mathrm{R}}$ are the volumes of the glassy and the rubbery states, respectively, extrapolated to $0 \mathrm{~K}$, and $\mathrm{v}_{\mathrm{f}}$ is the specific free volume at $\mathrm{T}=\mathrm{T}_{\mathrm{g}}$. 
According to the WLF (Williams-Landel-Ferry) equation, the expression of the fractional free volume as a function of temperature for a temperature above $\mathrm{T}_{\mathrm{g}}$ is given by [133]:

$$
f=f_{o}+\alpha_{f} .\left(T-T_{g}\right)
$$

where $\mathrm{f}$ is the free volume fraction. It is equal to the ratio of the average free volume in the polymer divided by the total sample volume $\mathrm{f}_{\mathrm{o}}$ is the free volume fraction at $\mathrm{T}_{\mathrm{g}}$ it is equal to 0.025 for all polymers $\alpha_{f}=\left(\alpha_{R}-\alpha_{G}\right)$ is the expansion coefficient of the free volume $\alpha_{\mathrm{G}}$ and $\alpha_{\mathrm{R}}$ represent the thermal expansion coefficients in the glassy and rubbery states, respectively

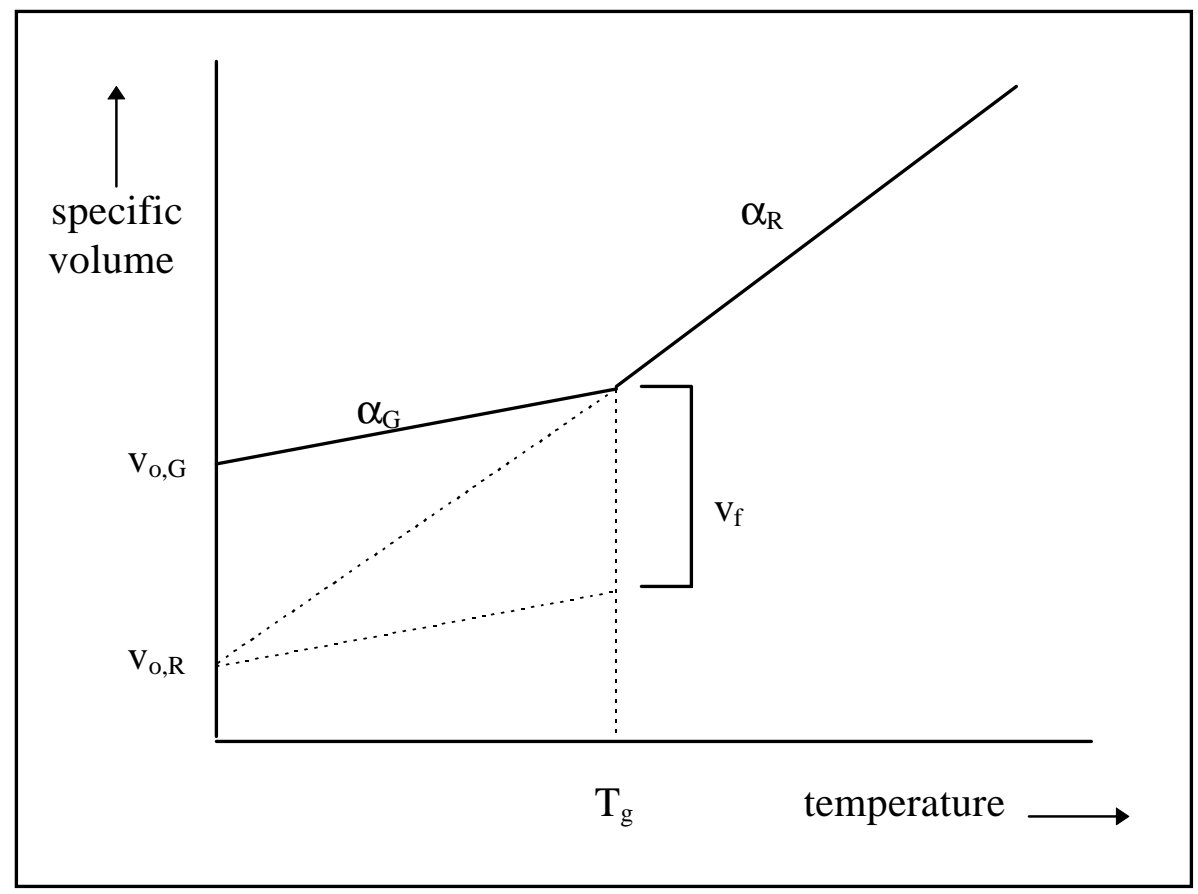

Figure 4.45: Diagram illustrating free volume (from [134]): 
The depression in the $\mathrm{T}_{\mathrm{g}}$ must be accounted for in the diffusion model. Diffusion in amorphous glassy polymers generally follows case II diffusion, whereas diffusion in rubbery polymers is expected to obey Fick's law. For the case II diffusion, the diffusion is very rapid compared to the polymer relaxation time. The diffusion coefficient is independent of the concentration profile, since it depends on the relaxation of the polymer material. The front of the interface moves at a constant velocity, separating the glassy from the plasticized region. On the other hand, the Fickian diffusion occurs when the rate of diffusion is much lower than the relaxation rate of the polymer. The case II is based on a random walk with strong interactions and a moving interface, whereas the Fickian behavior has no interactions. It has been established that when the uptake of a component is proportional to the time $\mathrm{t}$, the diffusion is referred as case II diffusion, whereas when the uptake is proportional to $t^{(1 / 2)}$, the diffusion is Fickian [102]. In order to check whether case II diffusion occured in our system, plots of the absorbance as a function of time and square root of time were generated. The plots observed for the peak at $1507 \mathrm{~cm}^{-1}$ at $\mathrm{T}=100^{\circ} \mathrm{C}$ are shown in Figures 4.46 and 4.47. The measured initial uptake part was directly proportional to time, as shown in Figure 4.46. However, if the first experimental point was removed in Figure 4.47, the initial uptake would have also been directly proportional to the square root of time. The fact that this line does not go through the origin is probably due to an experimental error rather than an induction period. The higher the temperature, the longer is the initial part proportional to the square root of time, as shown in Figure 4.48. Initially, the uptake changes linearly with time, as illustrated in Figure 4.46, after which there is a transition in the slope. If we take the first linear section of the curve and extrapolate it, the time at which it intercepts with the curve is called the duration time. The duration time decreased linearly with temperature, as demonstrated in Figure 4.49. 


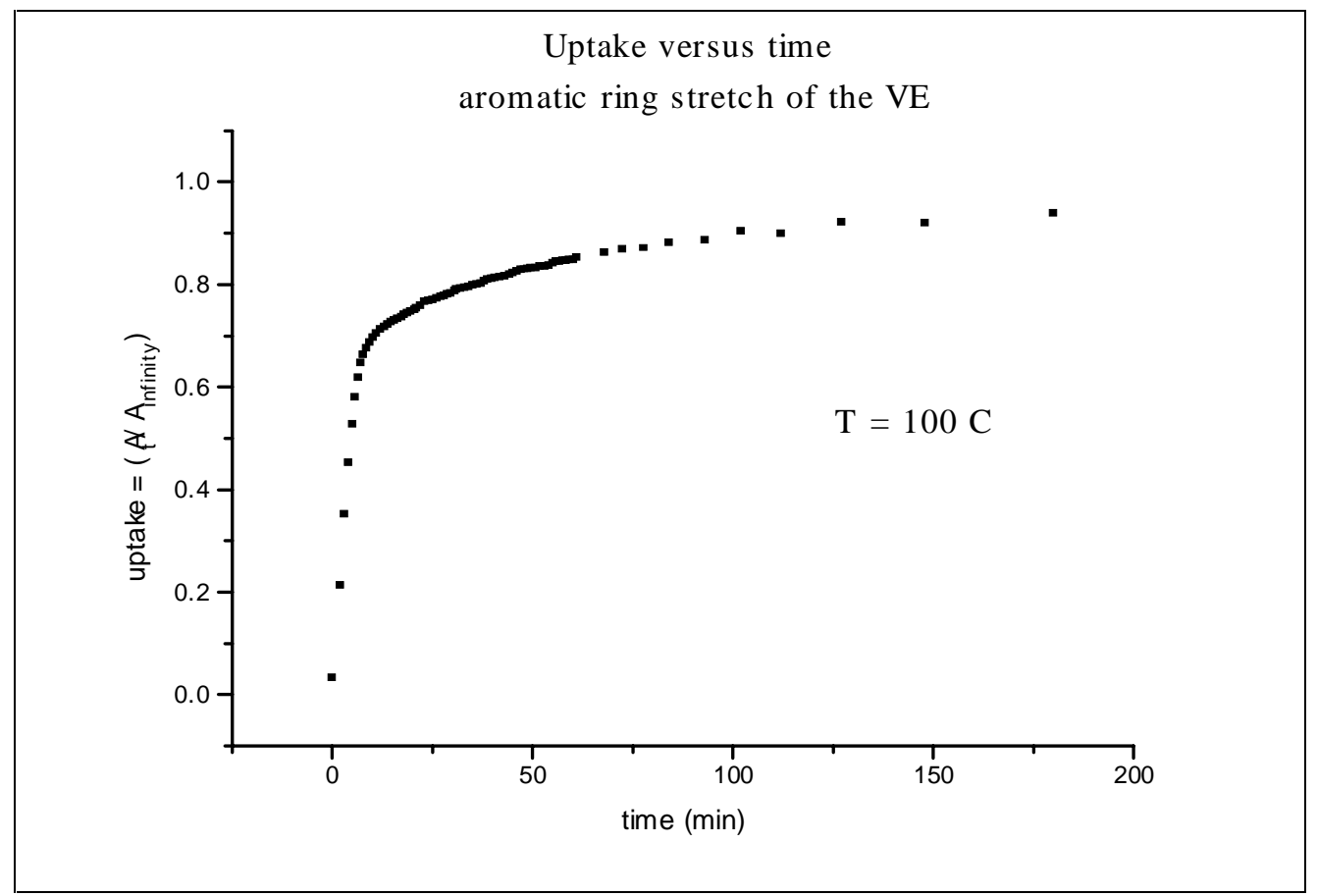

Figure 4.46: Uptake versus time for the peak at $1507 \mathrm{~cm}^{-1}$ at $\mathrm{T}=100^{\circ} \mathrm{C}$

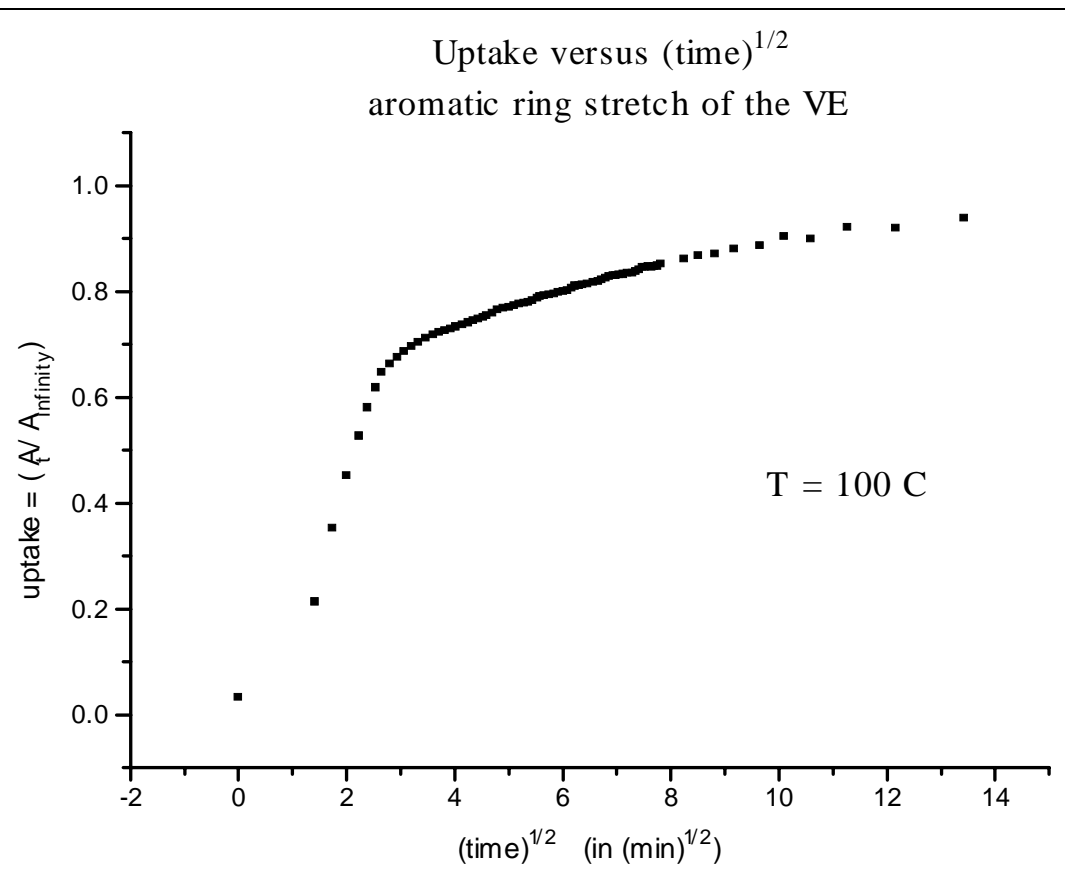

Figure 4.47: Uptake versus (time) ${ }^{1 / 2}$ for the peak at $1507 \mathrm{~cm}^{-1}$ at $\mathrm{T}=100^{\circ} \mathrm{C}$ 


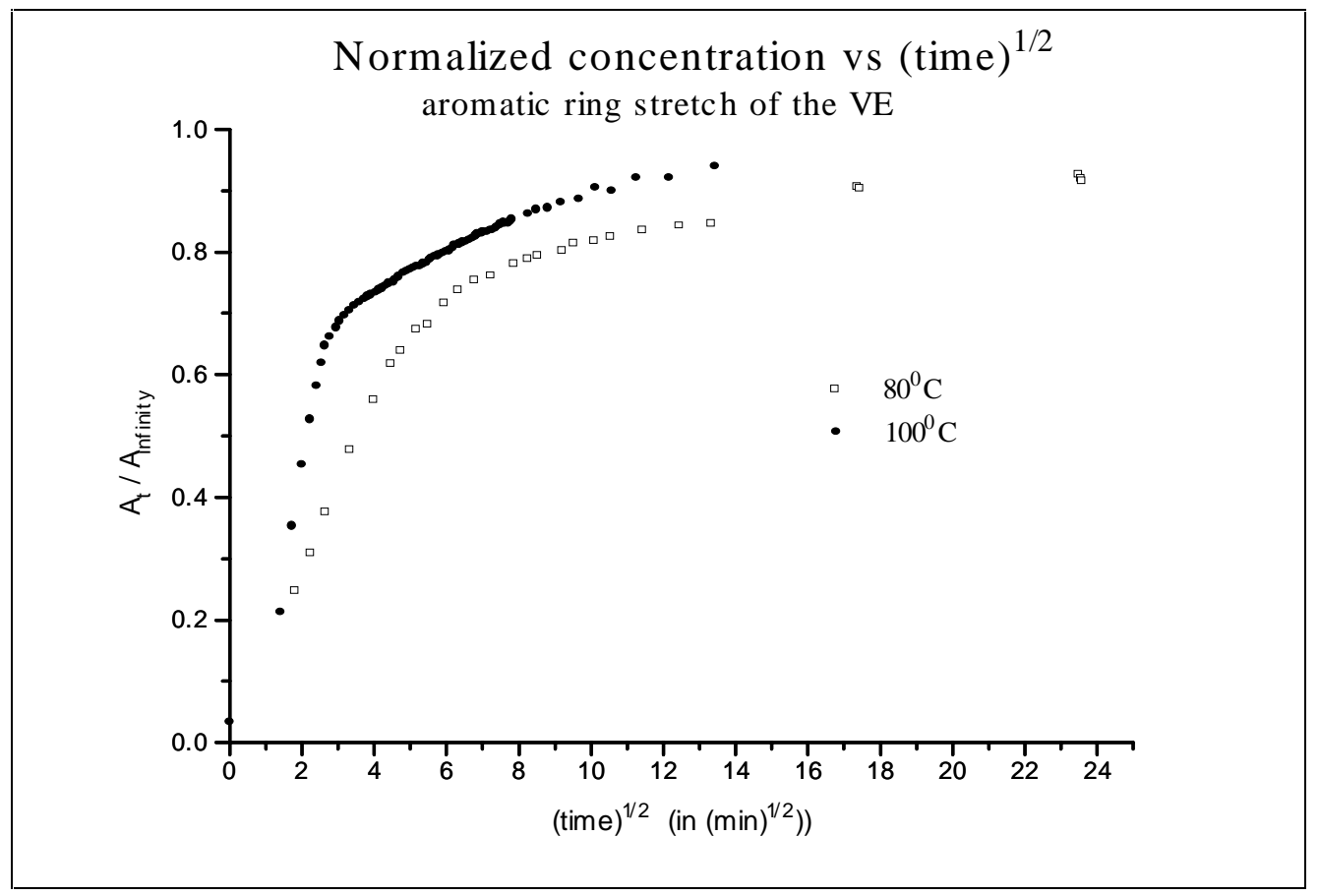

Figure 4.48: Uptake versus (time) ${ }^{1 / 2}$ at $80^{\circ} \mathrm{C}$ and $100^{\circ} \mathrm{C}$

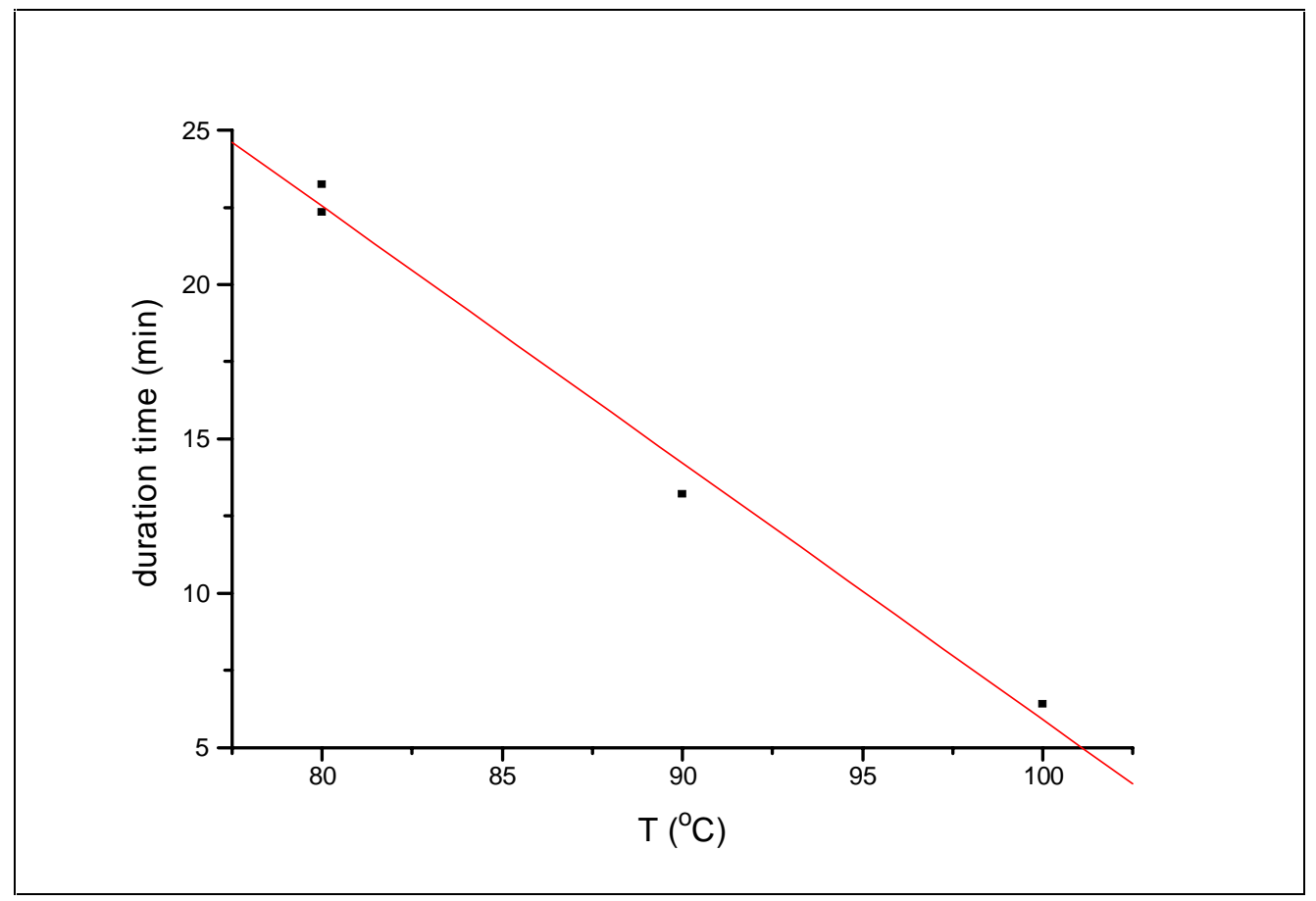

Figure 4.49: Duration time versus temperature 
Most researchers tried to model the case II diffusion by non-Fickian constitutive relations. However, a recent model introduced by Rossi, Pincus, and De Gennes [161], assumes that Fick's law can describe the transport in the glassy and the plasticized regions by two different diffusion coefficients. The only problem appears to be that the diffusion coefficient changes by many orders of magnitude at the interface between glassy and plasticized region. Therefore, instead of trying to develop a non-Fickian model, some modifications of Fick's law should be made.

Samus and Rossi [158] suggested a Fermi function form for the expression of the diffusion coefficient in the transition region between the glassy and the rubbery state. Its expression is given by:

$$
\mathrm{D}(\phi)=\mathrm{D}_{\mathrm{o}}+\frac{\mathrm{D}_{1}-\mathrm{D}_{\mathrm{o}}}{1+\exp (-\mathrm{L}(\phi-\tilde{\phi}))}
$$

where $\mathrm{D}(\phi)$ is the diffusion coefficient in the transition region

$\mathrm{D}_{\mathrm{o}}$ is the diffusion coefficient in the glassy phase

$\mathrm{D}_{1}$ is the diffusion coefficient in the rubbery phase

$\mathrm{L}$ is a parameter controlling the size of the transition region

$\phi$ is the concentration

$\widetilde{\phi}$ is the concentration above which plasticization occurs

Unfortunately, it was not possible to distinguish three regions in our case and curvefit the plots of the absorbance versus time we got, since the Fortran program "diff.for", given in Appendix $\mathrm{C}$, requires values of $\mathrm{A}_{\mathrm{o}}$ and $\mathrm{A}_{\propto}$, values which could not be estimated.

Sheamur et al. [96] suggested a model for the case where the concentration was close to the binodal curve and the temperature was above the glass transition temperature. Storey et al. [162] noticed that the diffusion coefficient for di-n-hexyl phthalate in PVC followed an Arrhenius relationship above and below the $\mathrm{T}_{\mathrm{g}}$. However, there was a change of activation energy at the $T_{\mathrm{g}}$. The activation energy for the diffusion was about three times 
lower in the glassy state. Three distinct stages of plasticizer uptake by PVC were then distinguished. Initially, a non-Fickian induction period, attributed to the change from a glassy to a rubbery material, was observed. Then a rapid uptake was noticed, and finally a swelling equilibrium was reached. In a following paper [146] concerning the diffusion of bis (2-ethylhexyl) phthalate in PVC, three stages were again observed, but the attributions were different. The first stage was considered as an induction period, the second one as the Fickian diffusion of plasticizer into the glassy polymer, and the third one as the Fickian diffusion into the rubbery PVC. A discontinuity was observed between the second and the third stages.

\subsubsection{3 - Swelling}

When the small molecules of VE penetrate into the PVP, the PVP swells, as established by the fast theory of diffusion, and outlined in Subsection 2.1.3. This swelling occurs because the PVP does not dissolve in the VE, and because the PVP is too sluggish to relax the density by diffusion. The swelling stress, induced by the invasion of the VE, leads to the movement of the PVP in the direction opposite to the VE flux. A very strong concentration dependence of the mutual diffusion coefficient may exist. In order to account for swelling, a three-dimensional equation, and not the one-dimensional equation (2.4.m) should have been used $[157,158]$. The swelling phenomenon could be confirmed with dynamic mechanical analysis [26].

\subsubsection{4 - Clustering}

As explained in Section 4.2, the VE can self-associate and also form hydrogen bonds with the PVP. However, a molecule that is interacting with itself or with a polymer does not simply diffuse through it. The Fickian diffusion does not consider the chemical interactions that occur in the system. The mobility of the penetrant may be reduced because of hydrogen bonding interactions, and the activation energy for diffusion may be higher. 


\subsubsection{5 - Concentration Dependent Diffusion Coefficient}

The diffusion model (2.4.s) used in modeling our data was developed assuming a constant mutual diffusion coefficient, and thus the values of the diffusion coefficients were concentration-averaged. However, these diffusion coefficients should be strongly dependent on concentration, because of the change in the glass transition temperature, and hence the change in the free volume of the system.

The one-dimensional Fick's law model would then have the expression:

$$
\left(\frac{\partial C}{\partial t}\right)=\frac{\partial}{\partial z}\left(D \cdot \frac{\partial C}{\partial z}\right)
$$

where $D$ is a function of concentration, and $D=f(z, C)$

One can assume that $\mathrm{D}(\mathrm{C}) \sim \mathrm{C}^{\mathrm{k}}$; as $\mathrm{C} \rightarrow 0, \mathrm{D}(\mathrm{C}) \rightarrow 0$ for $\mathrm{k}>0$, and $\mathrm{D}(\mathrm{C}) \rightarrow \propto$ for $\mathrm{k}<0$. The first case when $k>0$ is referred as hypodiffusive, whereas the second case when $k<0$ is called hyperdiffusive [29].

Barrie and Machin [163] chose several forms relating the diffusion coefficient and the concentration, some of them showing D decreasing with concentration and other functions showing D increasing with concentration. The functional dependence of D on concentration strongly affects the concentration profile in a polymeric membrane.

An exact relation between the mutual diffusion coefficient and the concentration has to be found in order to solve the differential equation (4.3.m). However, no theoretical models have been really verified. The fast and the slow theories, developed in Subsection 2.1.3, cannot be applied to our system, since those theories have been derived assuming that the self-diffusion coefficients are independent of concentration. However, this assumption is valid only provided that both polymers have similar $T_{g}$, and in the present case, the $T_{g}$ of the 
blend varies with composition. Moreover, it is likely that those theories apply only to the interdiffusion of polymers, and not in the case where a small molecule penetrates into a polymer. To have a better understanding of how a mutual diffusion coefficient and concentration are related, a method providing information on the self-diffusion coefficients should be used. The ATR spectroscopy should be employed in conjunction with another method which can measure self-diffusion coefficients. 


\section{CHAPTER 5}

\section{CONCLUSION AND FUTURE WORK}

The focus of this study was on the analysis of the molecular interdiffusion across a poly(vinyl pyrrolidone)/vinyl ester monomer (PVP/VE) interface. The primary objective of this work was to experimentally measure diffusion coefficients by Fourier Transform Infrared Attenuated Total Reflectance (FTIR-ATR) spectroscopy. Diffusion coefficients were determined by studying variations in infrared bands as a function of time, and by using a Fickian model. The values of the diffusion coefficients obtained were consistent with the range of values found in the literature for diffusion in polymers. The value of the diffusion coefficient increased with increasing the temperature, as expected by the Arrhenius equation. Hydrogen bonding interactions were also characterized.

However, the Fickian diffusion model used in this study did not seem to be really appropriate for this particular (PVP/VE) system. Emphasis should therefore be placed on developing a new model. The best way to further develop the analysis and to find a more suitable model would be to take into account plasticization, swelling and hydrogen bonding via a concentration dependent diffusion coefficient or an additional non-Fickian component in the model.

It would be interesting to confirm the results for the (PVP/VE) system by using variable angle of incidence for ATR spectroscopy, and thus probe different penetration depths, and also by studying a symmetrical configuration, i. e. by casting a film of VE

directly on a ATR crystal and by coating it with PVP. The interface region could also be examined by an optical microscope or EMP analysis, and thus an estimation of the interfacial thickness as a function of time could be obtained. 
Further analysis is needed to get more realistic data for the (sizing material/polymer matrix) interphase. As mentioned in the introduction, the vinyl ester monomer is usually diluted with $30 \mathrm{wt} \%$ of styrene. It would be interesting, as a first approach, to measure the diffusion of the styrene itself in the PVP, and thus to draw some conclusions on the importance of the nature of the styrene diffusant on the PVP (size, shape, chemical nature). Actually, it has been established that the PVP is incompatible with the polystyrene [164], and one can assume that the (PVP/styrene) system will probably have limited miscibility. Then, one should study the diffusion of the PVP and a mixture of (VE and styrene) at different concentrations. From these results, one should be able to determine the influence of a multicomponent solvent, as well as the influence of its composition. The ability of the VE to diffuse in the PVP can be drastically modified, indeed, by trace amounts of other molecules. Finally, the diffusion between the sizing material and the thermoset matrix could be investigated, and hence the mechanical properties of the composite. 


\section{REFERENCES}

1. M. Xie, C. L. Weitzsacker, M. Rich, L. T. Drzal, The interactions of vinyl ester matrix to carbon fiber surfaces, Proceedings of the 20th Annual "Anniversary" Meeting of the Adhesion Society, Hilton Head island, South Carolina, February 23-26, Lawrence T. Drzal, Henry P. Schreiber, Editors, 555-557 (1997).

2. J. J. Lesko, Interphase properties and their effects on the compression mechanics of polymeric composites, Dissertation, Department of Engineering Science and Mechanics, Virginia Polytechnic Institute and State University, August, 1994.

3. J. J. Lesko, A. Rau, J. S. Riffle, The effects of interphase properties on the durability of woven carbon/vinyl ester matrix composites, Proc. 10th Tech. Conf. of the American Society for Composites, 53-62 (1995).

4. B. K. Larson, L. T. Drzal, Glass fibre sizing/ matrix interphase formation in liquid composite moulding: effects on fibre/matrix adhesion and mechanical properties, Composites, 25, 7, 711-721 (1994).

5. S. S. Voyutskii, Autoadhesion and adhesion of high polymers, Wiley, New York, 1963.

6. J. J. Lesko, R. E. Swain, J. M. Cartwright, J. W. Chin, K. L. Reifsnider, D. A. Dillard, J. P. Wightman, Interphases developed from fiber sizings and their chemical structural relationship to composite compressive performance, J. Adhesion, 45, 43-57 (1994).

7 . C. D. Han, K.-W. Lem, Chemorheology of thermosetting resins IV. The chemorheology and curing kinetics of vinyl ester resin, Journal of Applied Polymer Science, 29, 1879-1902 (1984).

8. R. Blosser, J. Florio Jr., P. Donti, Continuous resin transfer molding ${ }^{\mathrm{TM}}$ of high quality, low cost, constant cross section, composite structural elements, 39th International SAMPE Symposium, April 11-14, 2, 1961-1972 (1994).

9. X. Dirand, B. Hilaire, E. Lafontaine, B. Mortaigne, M. Nardin, Crosslinking of vinyl ester matrix in contact with different surfaces, Composites, 25, 7, 645-652 (1994). 
10. R. E. Young, Unsaturated Polyester technology, Edited by Paul F. Bruins, Gordon \& Breach, New York, 1976.

11. H. Li, A. C. Rosario, S. V. Davis, T. Glass, T. V. Holland, R. M. Davis, J. J. Lesko, J. S. Riffle, J. Florio, Network formation of vinyl ester-styrene composite matrix resins, submitted.

12. M. E. Kelly, Unsaturated Polyester technology, Edited by Paul F. Bruins, Gordon \& Breach, New York, 1976.

13. G. Gündüz, Unsaturated polyesters resins (Overview), Polymeric Materials Encyclopedia, J. C. Salamone, editor, CRC Press, Boca Raton, 11, 8469-8476, 1996.

14. N. S. Broyles, Sizing and characterization of carbon fibers with aqueous waterdispersible polymeric interphases, Master Thesis, Department of Chemical Engineering, Virginia Polytechnic Institute and State University, February 1996.

15 . J. S. Riffle et al., Designed polymeric interphase materials, July 8-9, National Sience Foundation, Site Review, 1996.

16. H. T. Oyama, H. Li, S. V. Davis, J-P Wightman, J. S. Riffle, Interface between vinyl ester resin and poly(vinyl pyrrolidone), NSF report, 1996.

17 . J. I. Goldstein, D. E. Newbury, P. Echlin, D. C. Joy, A. D. Romig, Jr., C. E. Lyman, C. Fiori, E. Lifshin, Scanning electron microscopy and $x$-ray microanalysis, 2nd ed., Plenum Press, New York, 1992.

18. S. J. B. Reed, Electron microprobe analysis, 2nd ed., Cambridge University Press, Cambridge, 1993.

19. J. G. Van Alsten, Diffusion Measurements in Polymers Using IR Attenuated Total Reflectance Spectroscopy, Trends in Polymer Science, 3, 8, 272 (1995).

20 . J. G. Van Alsten and S. R. Lustig, Polymer Mutual Diffusion Measurements Using Infrared ATR Spectroscopy, Macromolecules, 25, 5069-5073 (1992).

21. E. Jabbari and N. A. Peppas, Use of ATR-FTIR To Study Interdiffusion in Polystyrene and Poly(vinyl methyl ether), Macromolecules, 26, 2175-2186 (1993).

22. S. R. Lustig, J. G. Van Alsten, and B. Hsiao, Polymer Diffusion in Semicrystalline Polymers 1. poly(ether imide)/Poly(aryl ether ketone ketone), Macromolecules, 26, 3885-3894 (1993). 
23. J. G. Van Alsten, S. R. Lustig, and B. Hsiao, Polymer Diffusion in Semicrystalline Polymers. 2. Atactic Polystyrene-d Transport into Atactic and Isotactic Polystyrene, Macromolecules, 28, 3672-3680 (1995).

24. E. Jabbari, N. A. Peppas, Molecular weight and polydispersity effects on interdiffusion at the interface between polystyrene and poly(vinyl methyl ether), Journal of Materials Science, 29, 3969-3978 (1994).

25 . E. Jabbari, N. A. Peppas, Comparison of interdiffusion at polystyrene-poly(vinyl methyl ether) and polystyrene-poly(isobutyl vinyl ether) interfaces, Polymer international, 38, 65-69, (1995).

26. E. Jabbari and N. A. Peppas, Temperature effects on interdiffusion at glassy/rubbery interfaces, J. Adhesion, 43, 101-119 (1993).

27. MRS Bulletin; A Publication of the Materials Research Society, 21, 1, 1-53, (1996).

28. Richard P. Wool, Polymer Interfaces- Structure and Strength, Hanser Publishers, 1995.

29. F. Brochard-Wyart, Fundamentals of Adhesion, Edited by Lieng-Huang Lee, Plenum Press, New York, 181-206, 1991.

30 . K. Binder, H. Sillescu, Diffusion polymer-polymer, Encyclopedia of Polymer Science and Engineering, 2nd Edition, H. F. Mark, N. M. Bikales, C. G. Overberger, G. Menges, Editors, John Wiley and Sons, New York, Supplement Volume, 297-315. 1989.

31. W. J. Koros, M. W. Hellums, Transport properties, Encyclopedia of Polymer Science and Engineering, 2nd Edition, H. F. Mark, N. M. Bikales, C. G. Overberger, G. Menges, Editors, John Wiley and Sons, New York, Supplement Volume, 724-802, 1989.

32. E. Jabbari, N. A. Peppas, Polymer-polymer Interdiffusion and Adhesion, J. M. S.-Rev. Macromol. Chem. Phys. , C34(2), 205-241 (1994).

33. J. Klein, Evidence for reptation in an entangled polymer melt, Nature, 271, 143-145 (1978).

34. J. Klein and B. J. Briscoe, Diffusion of large molecules in polymers: a measuring technique based on microdensitometry in the infrared, Polymer, 17, 6, 481-484 (1976). 
35. J. Klein and B. J. Briscoe, Technique for study of diffusion of large molecules in polymers based on infrared microdensitometry, Nature, 257, 386-387 (1975).

36. E. A. Jordon, R. C. Ball, A. M. Donald, L. J. Fetters, R. A. L. Jones, J. Klein, Mutual diffusion in blends of long and short entangled polymer chains, Macromolecules, 21, 235-239 (1988).

37. J. V. Seggern, S. Klotz and H.-J. Cantow, Probing the interface in blends of high and low molecular weight polystyrene, Macromolecules, 22, 8, 3328-3332 (1989).

38 . E. A. Jordan, R. C. Ball, A. M. Donald, L. J. Fetters, R. A. L. Jones and J. Klein, Mutual diffusion in blends of long and short entangled polymer chains, Macromolecules, 21, 1, 235-239 (1988).

39 . F. P. Price, P. T. Gilmore, E. L. Thomas, R. L. Laurence, Polymer/polymer diffusion. I. Experimental technique, J. Polym. Sci., Polym. Symp. Ed. , 63, 33-44 (1978).

40 . R. S. Raghava and R. W. Smith, Adhesion through molecular entanglements in polymer interfaces, J. Polym. Sci., Polym. Phys. Ed. , 27, 12, 2525-2551 (1989).

41 . P. T. Gilmore, R. Falabella and R. L. Laurence, Polymer/polymer diffusion. 2. Effect of temperature and molecular weight on macromolecular diffusion in blends of poly(vinyl chloride) and poly( $\varepsilon$-caprolactane), Macromolecules, 13, 4, 880-883 (1980).

42. S. Koizumi, H. Hasegawa and T. Hashimoto, Mutual diffusion of block polymer and homopolymer. Visualisation using microdomain as a probe, Macromolecules, 23, 11, 2955-2962 (1990).

43 . S. Klotz, J. Von Seggern, M. Kung and H.-J. Cantow, Imaging polymer interfaces by element specific electron microscopy and electron energy-loss spectroscopy, Polym. Commun. , 31, 8, 332-335 (1990).

44 . E. Jabbari and N. A. Peppas, Polym. Bull. , 27, 305 (1991).

45. E. Jabbari and N. A. Peppas, Proc. Adhes. Soc., 15, 113 (1992).

46. M. Ye, R. J. Composto and R. S. Stein, Modified optical Schlieren technique for measuring the mutual diffusion coefficient in polymer blends, Macromolecules, 23, 22, 4830-4834 (1990).

47 . B. B. Sauer and D. J. Walsh, Use of neutron reflection and spectroscopic ellipsometry for the study of the interface between miscible polymer films, Macromolecules, 24, 22, 5948-5955 (1991). 
48 . B. B. Sauer, D. J. Walsh, Effect of solvent casting on reduced entanglement density in thin films studied by ellipsometry and neutron scattering, Macromolecules, 27, 2, 432-440 (1994).

49 . J. Kanetakis and G. Fytas, Mutual diffusion in compatible polymer blends, Macromolecules, 22, 8, 3452-3458 (1989).

50 . P. J. Mills, P. F. Green, C. J. Palmstrom, J. W. Mayer and E. J. Kramer, Polydispersity effects on diffusion in polymers: concentration profiles of dpolystyrene measured by forward recoil spectrometry, J. Polym. Sci. Polym. Phys., 24, 1, 1-9 (1986).

51. F. Bruder, R. Brenn, B. Stuhn and G. R. Strobl, Interdiffusion in the partially miscible polymer blend of deuterated polystyrene and poly(styrene-co-bromo styrene), Macromolecules, 22, 12, $4434-4437$ (1989).

52. F. Bruder and R. Brenn, Measuring the binodial by interdiffusion in blends of deuterated polystyrene and poly(styrene-co-4-bromo-styrene), Macromolecules, 24, 20, 5552-5557 (1991).

53. P. F. Green and E. J.Kramer, Matrix effects on the diffusion of long chain polymers, Macromolecules, 19, 1108-1116 (1986).

54 . E. Kim, E. J. Kramer, J. O. Osby, D. J. Walsh, Mutual diffusion and thermodynamics in the blends of polystyrene and tetramethylbisphenol-A polycarbonate, Journal of Polymer Science: Part B: Polymer Physics, 33, 467-478 (1995).

55 . E. Kim, E. J. Kramer, W. C. Wu, P. D. Garrett, Diffusion in blends of poly(methyl methacrylate) and poly(styrene-co-acrylonitrile), Polymer, 35, 26, 5706-5715 (1994).

56 . R. J. Composto, E. J. Kramer, D. M. White, Fast macromolecules control mutual diffusion in polymer blends, Nature London, 328, 234-236 (1987).

57 . E. J. Kramer, P. Green, C. J. Palmstrøm, Interdiffusion and marker movements in concentrated polymer-polymer diffusion couples, Polymer, 25, 473-480 (1984).

58 . P. F. Green, C. J. Palmstrom, J. W. Mayer and E. J.Kramer, Marker displacement measurements of polymer-polymer interdiffusion, Macromolecules, 18, 501-507 (1985).

59. R. J. Composto and E. J. Kramer, Mutual diffusion studies of polystyrene and poly(xylenyl ether) using Rutherford backscattering spectrometry, Journal of materials science, 26, 2815-2822 (1991). 
60 . P. J. Mills and E. J. Kramer, Effects of molecular size on non-Fickian sorption in glassy polymers, J. Mater. Sci. , 21, 12, 4151-4156 (1986).

61. S. J. Whitlow and R. P. Wool, Investigation of diffusion in PS using secondary ion mass spectroscopy, Macromolecules, 22, 6, 2648-2652 (1989).

62. S. J. Whitlow, R. P. Wool, Diffusion of polymers at interfaces: a secondary ion mass spectroscopy study, Macromolecules, 24, 5926-5938 (1991).

63 . C. M. Roland and G. G. A. Bohm, Macromolecular diffusion and autoadhesion of polybutadiene, Macromolecules, 18, 6, 1310-1314 (1985).

64 . C. R. Bartels, B. Crist and W. W. Graessley, Self-diffusion coefficient in melts of linear polymers: chain lenght and temperature dependence for hydrogenated polybutadiene, Macromolecules, 17, 2702-2708 (1984).

65 . J. E. Anderson and J. H. Jon, Small-angle neutron scattering studies of diffusion in bulk polymers: experimental procedures, Macromolecules, 20, 7, 1544-1549 (1987).

66 . M. G. Brereton, E. W. Fischer, Ch. Herkt-Maerzky, K. Mortensen, Neutron scattering from a series of compatible polymer blends: significance of the Flory parameter, J. Chem. Phys. , 87, 10, 6144-6149 (1987).

67 . A. Karim, G. P. Felcher and T. P. Russell, Diffusion studies in polymer bilayers by neutron reflection, Polym. Prepr., 31, 2, 69-70 (1990).

68 . M. L. Femandez, J. S. Higgins, J. Penfold and C. Shackleton, Interfacial mixing of compatible polymers studied by neutron critical reflection, Polym. Prepr. , 31, 2, 71-72 (1990).

69. M. Stamm, G. Reiter, S. Huttenbach and M. Foster, Interfacial mixing of polymers as investigated by x-ray and neutron reflectometry, Polym. Prepr. , 31, 2, 73-74 (1990).

70 . J. Sokolov, X. Zhao, M. H. Rafailovich, J. M. Bloch, R. J. Composto, T. Mansfield, R. S. Stein, N. L. Yang, S. G. Greenbaum, R. A. L. Jones, E. J. Kramer and M. Sansone, X-ray fluorescence and reflectivity of PS/PBRS interfaces, Polym. Prepr. , 31, 2, 79-80 (1990).

71. P. P. Hong, F. J. Boerio, S. J. Clarson and S. D. Smith, An investigation of the interdiffusion of polystyrene and deuterated polystyrene using surface-enhanced Raman scattering, Macromolecules, 24, 17, 4770-4776 (1991). 
72 . G. Boven, R. H. G. Brinkhuis, E. J. Vorenkamp and A. J. Schouten, Interdiffusion of thin polymer layers studied by external reflection infrared spectroscopy, Macromolecules, 24, 4, 967-969 (1991).

73. E. Jabbari and N. A. Peppas, A model for interdiffusion at interfaces of polymers with dissimilar physical properties, Polymer, 36, 3, 575-586 (1995).

74. G. Rajagopalan, J. W. Gillepsie, Jr., S. H. McKnight, Interdiffusion in a poly-arylether-ether-ketone (PEEK)/epoxy system, ANTEC'96, Conference proceedings, May 5-10, Indianapolis, 1225-1230 (1996).

75 . H. Eklind, T. Hjertberg, Determination of Interdiffusion in Thin Polymer Films Using FTIR Reflection Absorption Spectroscopy, Macromolecules, 26, 5844-5851 (1993).

76. T. Buffeteau, B. Desbat, D. Eyquem, Attenuated total reflection Fourier transform infrared microspectroscopy: Theory and application to polymer samples, Vibrational Spectroscopy, 11, 29-36 (1996).

77. F. Bueche, W. M. Cashin and P. Debye, J. Chem. Phys., 20, 1956 (1952).

78. Y. Kumagai, H. Watanabe, K. Miyasaka and H. Hata, J. Chem. Eng. Japan, 12, 1-8 (1979).

79. P. Callaghan and D. Pinter, Self-diffusion of random-coil polystyrene determined by pulsed-field gradient nuclear magnetic resonance: dependence on concentration and molar mass, Macromolecules, 14, 1334-1340 (1981).

80 . S. H. Anastasiadis, K. Chrissopoulou, G. Fytas, G. Fleischer, S. Pispas, M. Pitsikalis, J. W. Mays, N. Hadjichristidis, Self-diffusivity in block copolymer solutions. 2. $\mathrm{A}_{2} \mathrm{~B}$ simple grafts, Macromolecules, 30, 8, 2445-2453 (1997).

81. R. Bachus and R. Kimmich, Molecular weight and temperature of self-diffusion coefficients in polyethylene and polystyrene melts investigated using a modified n.m.r. field-gradient technique, Polymer, 24, 964 (1983).

82. G. Fleischer, D. Geschke, J. Kärger and W. Heink, Peculiarities of self-diffusion studies on polymer systems by the NMR pulsed field gradient technique, J. Magn. Reson. , 65, 429-443 (1985).

83. G. Meier, G. Fytas, B. Momper, G. Fleischer, Interdiffusion in a homogeneous polymer blend far above its glass transition temperature, Macromolecules, 26, 5310-5315 (1993). 
84 . P. T. Callaghan and D. N. Pinder, Pulsed field gradient NMR, Directly determined polymer self diffusion coefficients compared with those derived from sedimentation or mutual diffusion, Polymer bulletin, 5, 305-309 (1981).

85. P. T. Callaghan and D. N. Pinder, Dynamics of entangled polystyrene solutions studied by pulsed field gradient nuclear magnetic resonance, Macromolecules, 13, 1085-1092 (1980).

86. L. Léger, H. Hervet and F. Rondelez, Self-diffusion in polymer solutions: a test for scaling and reptation, Phys. Rev. Letters, 42, 1681 (1979).

87 . M. Antonietti, J. Coutandin, R. Grütter and H. Sillescu, Diffusion of labeled macromolecules in molten polystyrenes studied by a holographic grating technique, Macromolecules, 17, 4, 798-802 (1984).

88. T. Lodge and B. Chapman, Applications of forced Rayleigh scattering to diffusion in polymeric fluids, Trends in polymer science, 5, 4, 122-128 (1997).

89 . L. M. Smith, B. A. Smith and H. M. MacConnell, Lateral diffusion of M-13 protein in model membranes, Biochemistry, 18, 11, 2256-2259 (1979).

90 . B. A. Smith, Measurement of diffusion in polymer films by fluorescence redistribution after pattern photobleaching, Macromolecules, 15, 2, 469-472 (1982).

91 . B. A. Smith, S. J. Mumby, E. T. Samulski and L. P. Yu, Concentration dependence of the diffusion of poly(propylene oxide) in the melt, Macromolecules, 19, 470-472 (1986).

92. U. Murschall, E. W. Fischer, C. Herkt-Maetzky, G. Fytas, Investigation of the mutual diffusion in compatible mixture of two homopolymers by photon correlation spectroscopy, Journal of polymer science: Part C: Polymer Letters, 24, 191-197 (1986).

93. T. Shiah and H. Morawetz, New fluorescence technique of characterizing polymer self-diffusion, Macromolecules, 17, $792-794$ (1984).

94 . R. W. Garbella and J. H. Wendorff, Small-angle x-ray studies on interdiffusion processes in blends of poly(methyl methacrylate) and poly(vinylidene fluoride), Makromol. Chem. Rapid Commun. , 7, 591-597 (1986).

95 . H. T. Oyama, J. J. Lesko, J. P. Wightman, Interdiffusion at the Interface between Poly(vinylpyrrolidone) and Epoxy, J. Polym. Science: Part B: Polym. Physics, 35, 331-346 (1997). 
96. T. E. Sheamur, A. S. Clough, D. W. Drew, M. G. D. Van Der Grinten, R. A. L. Jones, Interdiffusion of low molecular weight deuterated polystyrene and poly(methyl methacrylate), Macromolecules, 29, 7269-7275 (1996).

97. S. Yukioka, T. Inoue, Ellipsometric analysis of polymer-polymer interface, Polymer Communications, 32, 17-19 (1991).

98. S. Yukioka, K. Nagato, T. Inoue, Ellipsometric studies on mutual diffusion and adhesion development at polymer-polymer interfaces, Polymer, 33, 6, 1171-1176 (1992).

99 . P.-G. De Gennes, Dynamics of fluctuations and spinodal decomposition in polymer blends, J. Chem. Phys. , 72, 9, 4756-4763 (1980).

100 . F. Brochard, J. Jouffroy, P. Levinson, Polymer-polymer diffusion in melts, Macromolecules, 16, 1638-1641 (1983).

101 . K. J. Binder, Collective diffusion, nucleation, and spinodial decomposition in polymer mixtures, J. Chem. Phys. , 79, 12, 6387-6409 (1983).

102. J. Crank, The Mathematics of Diffusion, Second Edition, Clarendon Press, Oxford, 1975.

103. H. Sillescu, Relation of interdiffusion and tracer diffusion in polymer blends, Makromol. Chem., Rapid Commun., 8, 393-399 (1987).

104 . F. Feng, C. C. Han C, M. Takenaka, T. Hashimoto, Molecular weight dependence of mobility in polymer blends, Polymer, 33, 13, 2729-2739 (1992).

105 . A. Z. Akcasu, G. Nägele, R. Klein, Identification of modes in dynamic scattering from ternary polymer mixtures and interdiffusion, Macromolecules, 24, 4408-4422 (1991).

106 . M. G. Brereton, The dynamics of polymer blends: interdiffusion and the glass transition, Progress in colloid and polymer science, 91, 8-12 (1993).

107 . B. P. Straughan and S. Walker, editors, Spectroscopy, volume 2, John Wiley and Sons, 1976.

108. A. H. Fawcett, editor, Polymer spectroscopy, John Wiley and Sons, 1996.

109 . B. C. Smith, Fundamentals of Fourier Transform Infrared Spectroscopy, CRC Press, Inc., Boca Raton, 1996. 
110 . H. A. Willis, J. H. Van der Maas and R. G. J. Miller, editors, Laboratory methods in vibrational spectroscopy-3rd edition, John Wiley and Sons, 1987.

111. J. R. Durig, editor, Applications of FT-IR spectroscopy, Elsevier, 1990.

112. N. J. Harrick, Internal Reflection Spectroscopy, Harrick Scientific Corporation, New York, 1967.

113. F. M. Mirabella, Jr., editor, Internal Reflection Spectroscopy- Theory and Applications, Practical Spectroscopy Series, volume 15, 1993.

114 . F. M. Mirabella and N. J. Harrick, Internal Reflection Spectroscopy: Review and Supplement, Harrick Scientific Corporation, New York, 1985.

115. H. H. Kausch, K. Jud, Fracture mechanics and infrared measurements of the selfdiffusion of chains in polymer melt, Proc. 28th IUPAC Macromolecular Symposium, 717 (1982).

116 . N. A. Remizov, A. Y. Chalykh, V. Y. Popov, V. V. Lavrent'ev, Determination of the diffusion constants of liquids in polymers by sorption and by internal reflection spectroscopy, Polymer science U.S.S.R. , 24, 1853-1859 (1982).

117 . H. Brandth, P. Rieger, IR-ATR-spektrometrische Bestimmung des Diffusionskoeffizienten für das System Ethylacetat/Polyethylen, Experimentelle Technik der Physik, 32, 5, 413-416 (1984).

118 . J. R. Xu, C. M. Balik, Measurement of Diffusivities of Small Molecules in Polymers Using FTIR-ATR, Applied Spectroscopy, 42, 8, 1543-1548 (1988).

119 . T. P. Skourlis, R. L McCullough, Measurement of Diffusivity of a Liquid Diamine in Solid Epoxies Using Attenuated Total Reflectance Infrared Spectroscopy (FTIR-ATR), Journal of Applied Polymer Science, 52, 1241-1248 (1994).

120 . G. T. Fieldson and T. A. Barbari, Analysis of Diffusion in Polymers Using Evanescent Field Spectroscopy, AIChE Journal, 41, 4, 795-804 (1995).

121 J. G. Van Alsten and J. C. Coburn, Structural Effects on the Transport of Water in Polyimides, Macromolecules, 27, 3746-3752 (1994).

122 . K. M. Immordino, S. H. McKnight, J. W. Gillespie, Jr., Characterization of Polysulfone-Epoxy/Amine Interphase For Bonding Thermoplastic Composites, Proceedings of the 19th annual Meeting of the Adhesion Society, Myrtle Beach, South Carolina, February 18-21, Thomas C. Ward, Editor, 449-452 (1996). 
123 . R. A. Shick, J. L. Koenig, H. Ishida, Depth profiling of stratified layers using variable-angle ATR, Applied Spectroscopy, 50, 8, 1082-1088 (1996).

124 . E. K. Rideal, Diffusion in and through solids, The Cambridge Series of Physical Chemistry, 1951.

125. G. T. Fieldson and T. A. Barbari, The use of FTIR-ATR spectroscopy to characterize penetrant diffusion in polymers, Polymer, 34, 6, 1146-1153 (1993).

126. K. Cogan Farinas, L. Doh, S. Venkatraman, and R. O. Potts, Characterization of Solute Diffusion in a Polymer Using ATR-FTIR Spectroscopy and Bulk Transport Techniques, Macromolecules, 27, 5220-5222 (1994).

127. P. Y. Furlan, Hydrogenated Polybutadiene Morphology and Its Effects on Small Molecule Diffusion, Macromolecules, 25, 6516-6522 (1992).

128 . R. P. Semwal, S. Banerjee, L. R. Chauhan, A. Bhattacharya, and N. B. S. N. Rao, Study of Diffusion and Sorption of Bis-(2-chloroethyl)sulfide (SM) and Bis-(2chloroethyl)ether (OM) Through Polypropylene (PP) and Biaxial-Oriented Polypropylene (BOPP) Films by the FTIR-ATR Spectroscopic Method, Journal of Applied Polymer Science, vol. 60, 29-35 (1996).

129 . S. U. Huong, T. A. Barbari, J. M. Sloan, Diffusion of methyl ethyl ketone in polyisobutylene: comparison of spectroscopic and gravimetric techniques, Journal of Polymer Science: Part B: Polymer Physics, 35, 8, 1261-1267 (1997).

130 . K. M. Immordino, S. H. McKnight, J. W. Gillepsie, Jr., In situ evaluation of the diffusion of epoxy and amine in thermoplastic polymers, ANTEC'96, Conference proceedings, May 5-10, Indianapolis, 1214-1218 (1996).

131 . K. S. Kwan, T. C. Ward, Investigating the role of penetrant structure on its diffusion through a thermoset adhesive, Proceedings of the 20th Annual "Anniversary" Meeting of the Adhesion Society, Hilton Head island, South Carolina, February 23-26, Lawrence T. Drzal, Henry P. Schreiber, Editors, 585-587 (1997).

132 . B. V. Robinson, F. M. Sullivan, J. F. Borzelleca, S. L. Schwartz, PVP-A critical review of the kinetics and toxicology of polyvinylpyrrolidone (Povidone), Lewis Publishers, 1990.

133. E. S. Barabas, N - Vinyl amide polymers, Encyclopedia of Polymer Science and Engineering, 2nd ed., H. F. Mark, N. M. Bikales, C. G. Overberger, G. Menges, Editors, John Wiley and Sons, New York, volume 17, 204, 1989. 
134 . L. H. Sperling, Introduction to physical polymer science, Second edition, John Wiley and Sons, 1992.

135 . J. C. Seferis, Refractive indices of polymers, Polymer Handbook, Third Edition, J. Brandrup, E.H. Immergut, Editors, Wiley Interscience, VI/461, 1989.

136. A. W. Adamson, Physical Chemistry of Surfaces, Fifth Edition, Wiley Interscience, 1990.

137 . V. Offermann, P. Grosse, M. Feuerbacher, G. Dittmar, Experimental aspects of attenuated total reflectance spectroscopy in the infrared, Vibrational Spectroscopy, 8, 135-140 (1995).

138 . N. P. G. Roeges, A guide to the complete interpretation of infrared spectra of organic structures, John Wiley and Sons, 1994.

139 . N. L. Alpert, W. E. Keiser, H. A. Szymanski, IR-Theory and Practice of Infrared Spectroscopy, Plenum/Rosetta, 1973.

140 . M. Ganem, B. Mortaigne, V. Bellenger, J. Verdu, Influence of the styrene ratio on the copolymerization kinetics of dimethacrylate of diglycidyl ether of bisphenol A vinylester resins crosslinked with styrene, J. M. S. - Pure App. Chem. , A30(11), 829-848 (1993).

141 . C. H. Papadimitriou, K. Steiglitz, Combinatorial optimization, Algorithms and complexity, Prentice - Hall, Inc., Englewood Cliffs, 1982.

142 . P. Chih-ch'iian, Binary molecular diffusion coefficient, Journal of Applied Chemistry of the USSR, 62, 11, 2320-2333 (1989).

143 . M. D. Lechner, D. G. Steinmeier, Sedimentation coefficients, diffusion coefficients, partial specific volumes, frictional ratios, and second virial coefficients of polymers in solution, Polymer Handbook, Third Edition, J. Brandrup, E.H. Immergut, Editors, Wiley Interscience, VII/77, 1989.

144. H. T. Oyama, personal communication.

145 . D. Arnould, R. L. Laurence, Size effects on solvent diffusion in polymers, Industrial Engineering Chemistry Research, 31, 218-228 (1992).

146. R. F. Storey, K. A. Mauritz, B. B. Cole, Diffusion of bis (2-ethylhexyl) phthalate above and below the glass transition temperature of poly(vinyl chloride), Macromolecules, 24, 450-454 (1991). 
147 . I. Quijada-Garrido, J. M. Barrales-Rienda, G. Frutos, Diffusion of Erucamide (13-cis-Docosenamide) in Isotactic Polypropylene, Macromolecules, 29, 7164-7176 (1996).

148 . P. T. Gilmore, R. Falabella, R. L. Laurence, Polymer/polymer diffusion. 2. Effect of temperature and molecular weight on macromolecular diffusion in blends of poly(vinyl chloride) and poly(e-caprolactone), Macromolecules, 13, 4, 880-883 (1980).

149 . S. Wu, H.-K. Chuang, C. Dae Han, Diffuse interface between polymers: structure and kinetics, Journal of polymer science: Polymer physics edition, 24, 143-159 (1986).

150 . V. Janarthanan, G. Thyagarajan, Miscibility studies in blends of poly(N-vinyl pyrrolidone) and poly(methyl methacrylate) with epoxy resin: a comparison, Polymer, 33, 17, 3593-3597 (1992).

151. M. M. Coleman, J. F. Graf, P. C. Painter, Specific interactions and the miscibility of polymer blends, Technomic Publishing Co., Inc., Lancaster, 1991.

152. Sadtler Research Laboratories, Infrared spectra handbook of common organic solvents, Division of Bio-Rad Laboratories, Inc., Philadelphia, Pennsylvania, 1983.

153 . R. T. Graf, J. L. Koenig, H. Ishida, Comparison of FTIR transmission, specular reflectance, and attenuated total reflectance spectra of polymers, Polymer preprints, division of polymer chemistry, American Chemical Society, 25, 2, 188-189 (1984).

154 . J. C. Seferis, Refractive indices of polymers, Polymer Handbook, Third Edition, J. Brandrup, E.H. Immergut, Editors, Wiley Interscience, VI/451, 1989.

155 . R. T. Graf, J. L. Koenig, H. Ishida, Optical constant determination of thin polymer films in the infrared, Applied Spectroscopy, 39, 3, 405-408 (1985).

156 . H. Ishida, Quantitative surface FTIR spectroscopic analysis of polymers, Rubber Chemistry and Technology, 60, 3, 497 (1987).

157 . G. Rossi, Macroscopic description of solvent diffusion in polymeric materials, Trends in Polymer Science, 4, 10, 337-342 (1996).

158 . M. A. Samus and G. Rossi, Methanol Absorption in Ethylene-Vinyl Alcohol Copolymers: Relation between Solvent Diffusion and Changes in Glass Transition temperature in Glassy Polymeric Materials, Macromolecules, 29, 2275-2288 (1996). 
159 . J. K. Sears, N. W. Touchette, Plasticizers, Encyclopedia of Polymer Science and Engineering, 2nd Edition, H. F. Mark, N. M. Bikales, C. G. Overberger, G. Menges, Editors, John Wiley and Sons, New York, Supplement Volume, 568-647, 1989.

160 . J. K. Sears, N. W. Touchette, J. R. Darby, Plasticizers, Applied Polymer Science, R. W. Tess and G. W. Poehlein, Editors, ACS Symposium Series, Washington D. C., 611-641, 1985.

161 . G. Rossi, P. A. Pincus, P.-G. De Gennes, A Phenomenological Description of Case-II Diffusion in Polymeric Materials, Europhysics Letters, 32, 5, 391-396 (1995).

162 . R. F. Storey, K. A. Mauritz, B. D. Cox, Diffusion of various dialkyl phthalate plasticizers in PVC, Macromolecules, 22, 1, 289-294 (1989).

163 . J. A. Barrie and D. Machin, Concentration dependence of the time lag for diffusion, Journal of polymer science, A2, 5, 1300-1304 (1967).

164 . J. R. Isasi, L. Cesteros, I. Katime, Study of the miscibility and specific interactions between poly(1-vinyl-2-pyrrolidone) and poly(vinyl formal), Polymer, 34, 11, 2374-2379 (1993). 


\section{APPENDICES}

Appendix A: Nomenclature

Appendix B: Example of results obtained by curvefitting

the peak at $1508 \mathrm{~cm}^{-1}$ at $\mathrm{T}=80^{\circ} \mathrm{C}$

Appendix C: The Fortran program "diff.for"

Appendix D: The Fortran program "curvefit.for"

Appendix E: The Fortran program "conc2.for" 


\section{APPENDIX A}

\section{NOMENCLATURE}

The following terms have been published by the American Society for Testing and Materials (ASTM) in the 1967 ASTM Book of Standards.

\section{Internal Reflection Spectroscopy (IRS)}

The technique of recording optical spectra by placing a sample material in contact with a transparent medium of greater refractive index and measuring the reflectance (single or multiple) from the interface, generally at angles of incidence greater than the critical angle.

\section{Attenuated Total Reflection (ATR)}

Reflection which occurs when an absorbing coupling mechanism acts in the process of total internal reflection to make the reflectance less than unity.

Note: In this process, if an absorbing sample is placed in contact with the reflecting surface, the reflectance for total internal reflection will be attenuated to some value between greater than zero and unity in regions of the spectrum where absorption of the radiant power can take place.

\section{Internal Reflection Element (IRE)}

The transparent optical element used in Internal Reflection Spectroscopy for establishing the conditions necessary to obtain the internal reflection spectra of materials. 


\section{APPENDIX B}

EXAMPLE OF RESULTS OBTAINED BY CURVEFITTING THE PEAK AT $1508 \mathrm{CM}^{-1}$ AT $\mathrm{T}=80^{\circ} \mathrm{C}$

The peak at $1508 \mathrm{~cm}^{-1}$ corresponds to the aromatic ring stretch of the VE.

The best fit was generally obtained with a Gaussian peak.

\begin{tabular}{|c|c|c|c|c|c|c|c|}
\hline file & $\mathrm{t}(\min )$ & $\mathrm{X}^{2}$ & $\mathrm{~cm}^{-1}$ & height & $\mathrm{cm}^{-1}$ & height & $\mathrm{T}$ \\
\hline A1 & 1.17 & noise & & & & & 49 \\
\hline $\mathrm{A} 2$ & 4 & noise & & & & & 76 \\
\hline A3 & 6.17 & noise & & & & & 79 \\
\hline A4 & 12.25 & noise & & & & & 80 \\
\hline$\widehat{A 5}$ & 19.5 & 0.436172 & 1506.86 & 0.00594763 & 1493.45 & 0.0934199 & 78 \\
\hline A6 & 28.25 & 0.619334 & 1508.66 & 0.0742403 & 1493.92 & 0.0931828 & 80 \\
\hline A7 & 30 & 0.742594 & 1508.67 & 0.0924043 & 1494.07 & 0.0925582 & 81 \\
\hline A8 & 32 & 0.79859 & 1508.66 & 0.11265 & 1494.19 & 0.0921158 & 80 \\
\hline A9 & 36 & 0.666602 & 1508.63 & 0.142598 & 1494.47 & 0.092062 & 80 \\
\hline$\overline{B 1}$ & 40.75 & 0.103324 & 1508.61 & 0.167071 & 1494.8 & 0.092713 & 80 \\
\hline $\bar{B} 2$ & 44.83 & 0.137663 & 1508.44 & 0.184615 & 1494.91 & 0.0905812 & 80 \\
\hline B3 & 47.33 & 0.557941 & 1508.59 & 0.191171 & 1495.29 & 0.0944785 & 79 \\
\hline B4 & 51.58 & 0.491155 & 1508.52 & 0.201435 & 1495.38 & 0.0947849 & 80 \\
\hline B5 & 55 & 0.444148 & 1508.59 & 0.204018 & 1495.75 & 0.098046 & \\
\hline B6 & 60 & 0.444208 & 1508.51 & 0.214243 & 1495.8 & 0.0976184 & \\
\hline B7 & 64.75 & 0.477401 & 1508.48 & 0.220703 & 1495.92 & 0.0980001 & \\
\hline B8 & 70.67 & 0.467842 & 1508.47 & 0.225304 & 1496.16 & 0.0993705 & \\
\hline B9 & 77.17 & 0.423184 & 1508.48 & 0.227623 & 1496.49 & 0.10198 & \\
\hline $\mathrm{C} 1$ & 86.5 & 0.442267 & 1508.45 & 0.233328 & 1496.73 & 0.103494 & \\
\hline $\mathrm{C} 2$ & 92.67 & 0.420859 & 1508.43 & 0.235909 & 1496.9 & 0.104592 & \\
\hline C3 & 97.42 & 0.0718378 & 1508.42 & 0.237495 & 1497.02 & 0.10533 & \\
\hline
\end{tabular}




\begin{tabular}{|l|r|r|r|r|r|r|r|}
\hline C4 & 109.5 & 0.423067 & 1508.4 & 0.239659 & 1497.17 & 0.106548 & \\
\hline C5 & 115.42 & 0.419163 & 1508.37 & 0.243291 & 1497.34 & 0.107608 & \\
\hline C6 & 126.33 & 0.378431 & 1508.34 & 0.24471 & 1497.53 & 0.10904 & \\
\hline C7 & 136 & 0.395282 & 1508.34 & 0.246456 & 1497.67 & 0.109452 & \\
\hline C8 & 155 & 0.398331 & 1508.29 & 0.249638 & 1497.91 & 0.112634 & \\
\hline C9 & 179.83 & 0.361753 & 1508.25 & 0.252026 & 1498.21 & 0.115073 & \\
\hline D1 & 202.5 & 0.359331 & 1508.19 & 0.25303 & 1498.55 & 0.121441 & \\
\hline D2 & 326.25 & 0.377638 & 1508.04 & 0.271071 & 1498.53 & 0.123076 & \\
\hline D3 & 328.25 & 0.386393 & 1508.04 & 0.270165 & 1498.58 & 0.123532 & \\
\hline D4 & 576.33 & 0.352657 & 1507.92 & 0.276875 & 1499.07 & 0.129909 & \\
\hline D5 & 579 & 0.0632274 & 1507.99 & 0.274927 & 1499.22 & 0.128569 & \\
\hline D6 & 580 & 0.361031 & 1507.97 & 0.273771 & 1499.25 & 0.129575 & \\
\hline
\end{tabular}




\section{APPENDIX C \\ THE FORTRAN PROGRAM "DIFF.FOR" \\ Program written with the help of Dr. Sukhtej S. Dhingra}

CCCCCCCCCCCCCCCCCCCCCCCCCCCCCCCCCCCCCCCCCCCCCCCCCCCCCC $\mathrm{C}$ DIFF.FOR C

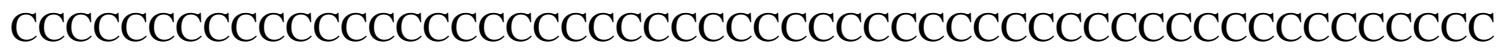

c program to get D and Ainfinity for diffusion in polymers

c Program for curve fitting using two parameter SIMPLEX optimization algorithm

program simplex

dimension $\mathrm{C}(5), \mathrm{E}(5), \mathrm{P}(5,5), \mathrm{R}(5), \mathrm{X}(5)$

integer $\mathrm{H}$

real K

c initial values

$\mathrm{N}=2$

$\mathrm{K}=0.20$

$\mathrm{N} 1=\mathrm{N}+1$

c set initial guesses for D and Ainfinity

c $\quad X(1)$ is the diffusion coefficient D

c $\mathrm{X}(2)$ is the $\mathrm{A}$ (infinity) variable

c $\quad X(1)$ and (X2) have to be in the same order of magnitude

c the exponents of $\mathrm{D}$ and Ainfinity will be defined later in this program

$\mathrm{X}(1)=145.5012$

$X(2)=320.5925$

c Initialise simplex

do $\mathrm{j}=1, \mathrm{~N}$

$P(1, j)=X(j)$

end do 


$$
\begin{aligned}
& \text { do } i=2, N 1 \\
& \text { do } j=1, N \\
& P(i, j)=X(j) \\
& \text { end do }
\end{aligned}
$$

c set the step below

c take first 1.5 , then $1.1,1.01$, and finally 1.001

$$
\begin{aligned}
& \mathrm{P}(\mathrm{i}, \mathrm{i}-1)=1.001 * \mathrm{X}(\mathrm{i}-1) \\
& \text { if }(\operatorname{abs}(\mathrm{X}(\mathrm{i}-1)) \cdot 1 \mathrm{t} .(1.0 \mathrm{E}-12)) \text { then }
\end{aligned}
$$$$
\text { write }\left(6,{ }^{*}\right) \text { 'Reaching lower limit' }
$$$$
\mathrm{P}(\mathrm{i}, \mathrm{i}-1)=0.00001
$$

end if

end do

c Find PL, PH

$11 \mathrm{~L}=1$

$\mathrm{H}=1$

do $\mathrm{i}=1, \mathrm{~N} 1$

do $\mathrm{j}=1, \mathrm{~N}$

$$
X(j)=P(i, j)
$$

end do

c $\quad$ write $(6, *)$ 'Hi!'

$\mathrm{E}(\mathrm{i})=\operatorname{error}(\mathrm{X})$

if ( $\mathrm{E}(\mathrm{i}) \cdot \mathrm{lt} \cdot \mathrm{E}(\mathrm{L})) \mathrm{L}=\mathrm{i}$

if $(\mathrm{E}(\mathrm{i}) \cdot \mathrm{gt} \cdot \mathrm{E}(\mathrm{H})) \mathrm{H}=\mathrm{i}$

end do

c find $\mathrm{PNH}$

$71 \mathrm{NH}=\mathrm{L}$

do $\mathrm{i}=1, \mathrm{~N} 1$

if $((\mathrm{E}(\mathrm{i}) \cdot \mathrm{ge} . \mathrm{E}(\mathrm{NH}))$.and.(i.ne.H)) then

$\mathrm{NH}=1$

end if

end do

c calculate centroid

$$
\begin{aligned}
& \text { do } \mathrm{j}=1, N 1 \\
& \mathrm{C}(\mathrm{j})=-\mathrm{P}(\mathrm{H}, \mathrm{j}) \\
& \text { do } \mathrm{i}=1, \mathrm{~N} 1 \\
& \mathrm{C}(\mathrm{j})=\mathrm{C}(\mathrm{j})+\mathrm{P}(\mathrm{i}, \mathrm{j})
\end{aligned}
$$

end do

$\mathrm{C}(\mathrm{j})=\mathrm{C}(\mathrm{j}) / \mathrm{N}$

end do 
c reflect

61 do $\mathrm{j}=1, \mathrm{~N}$

$\mathrm{R}(\mathrm{j})=1.9985 * \mathrm{C}(\mathrm{j})-0.9985 * \mathrm{P}(\mathrm{h}, \mathrm{j})$

end do

c write $\left(6,{ }^{*}\right)$ 'REFLECTING !'

$\mathrm{ER}=\operatorname{error}(\mathrm{R})$

c reflect again

if (ER.lt.E(L)) go to 41

if (ER.gt.E(H)) go to 51

21 do $\mathrm{j}=1, \mathrm{~N}$

$\mathrm{P}(\mathrm{H}, \mathrm{j})=\mathrm{R}(\mathrm{j})$

end do

$\mathrm{E}(\mathrm{H})=\mathrm{ER}$

if (ER.gt.E(NH)) go to 61

$\mathrm{H}=\mathrm{NH}$

go to 71

c Expand

$41 \mathrm{~L}=\mathrm{H}$

do $\mathrm{j}=1, \mathrm{~N}$

$\mathrm{X}(\mathrm{j})=1.95 * \mathrm{R}(\mathrm{j})-0.95 * \mathrm{C}(\mathrm{j})$

end do

c write $\left(6,{ }^{*}\right)$ 'EXPANDING !'

$\mathrm{EX}=\operatorname{error}(\mathrm{X})$

if (EX.lt.ER) go to 81

do $\mathrm{j}=1, \mathrm{~N}$

$\mathrm{P}(\mathrm{L}, \mathrm{j})=\mathrm{R}(\mathrm{j})$

end do

$\mathrm{E}(\mathrm{L})=\mathrm{ER}$

goto 91

81 do $\mathrm{j}=1, \mathrm{~N}$

$P(L, j)=X(j)$

end do

$\mathrm{E}(\mathrm{L})=\mathrm{EX}$

91 do $\mathrm{j}=1, \mathrm{~N}$

c write $\left(3,{ }^{*}\right) \mathrm{P}(\mathrm{L}, \mathrm{j})$

end do

c write $\left(3,{ }^{*}\right) \mathrm{E}(\mathrm{L})$

goto 71 


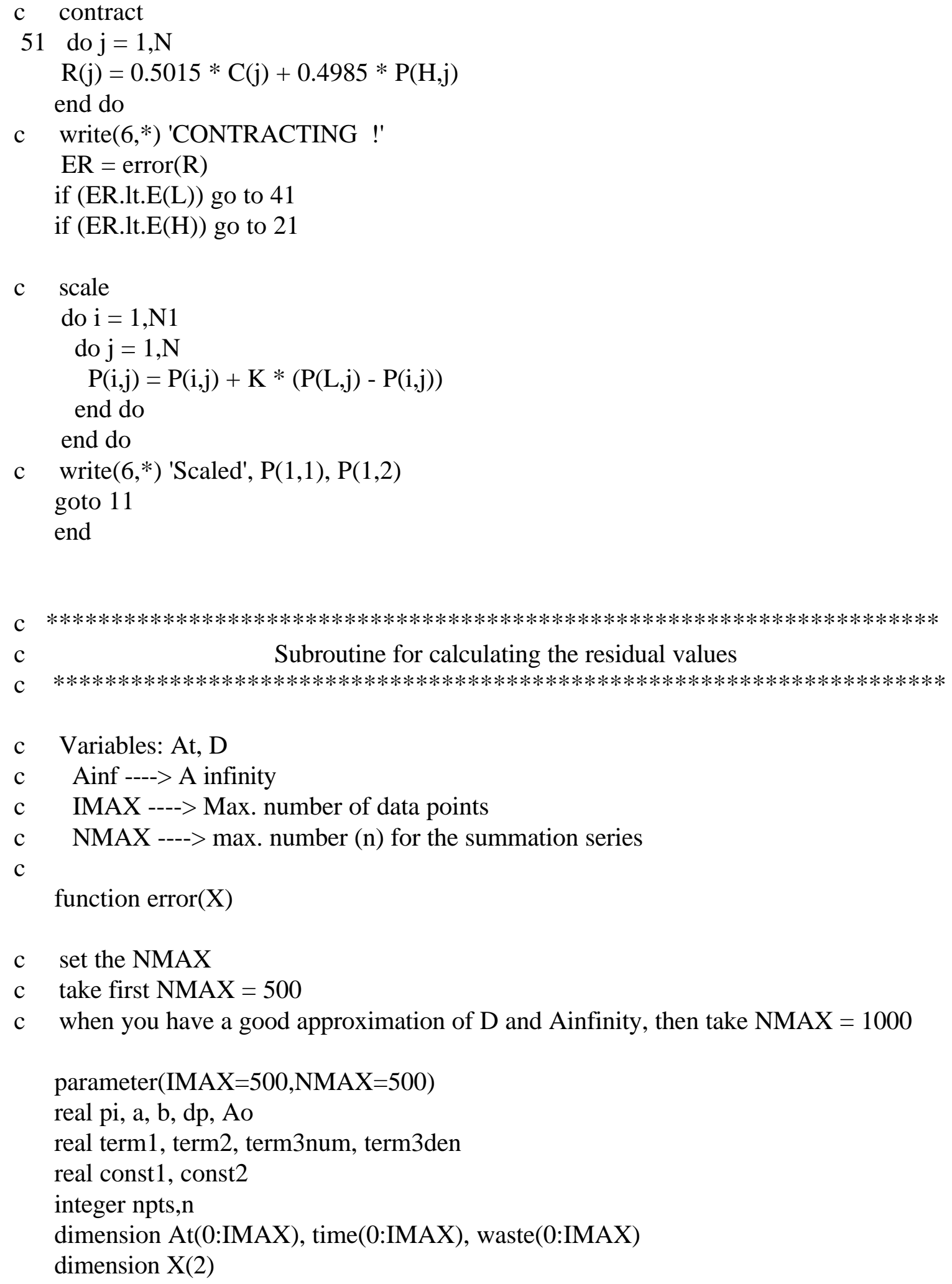


c set the parameters

c a, b and dp are in $\mathrm{m}$

$\mathrm{pi}=3.141592654$

$\mathrm{a}=0.0005$

$\mathrm{b}=0.000003$

$\mathrm{dp}=1.2540932 \mathrm{E}-06$

Ao $=0.68329$

c MAKE SURE YOUR DATA FILE IS NAMED "fort.99"

npts $=0$

do $\mathrm{j}=0,500$

$\operatorname{read}\left(99,{ }^{*}\right.$, end $\left.=101\right)$ time(j), waste(j), At(j)

c change the origin for the time

time $(\mathrm{j})=$ time $(\mathrm{j})-40$

npts $=$ npts +1

end do

101 continue

rewind(99)

c set the exponents of $\mathrm{X}(1)$ and $\mathrm{X}(2)$

c $\quad \mathrm{D}$ is in $\mathrm{m} 2 . \mathrm{s}-1$

$\mathrm{D}=\mathrm{X}(1)^{*} 1.00 \mathrm{E}-15$

Ainf $=\mathrm{X}(2) * 1.00 \mathrm{E}-03$

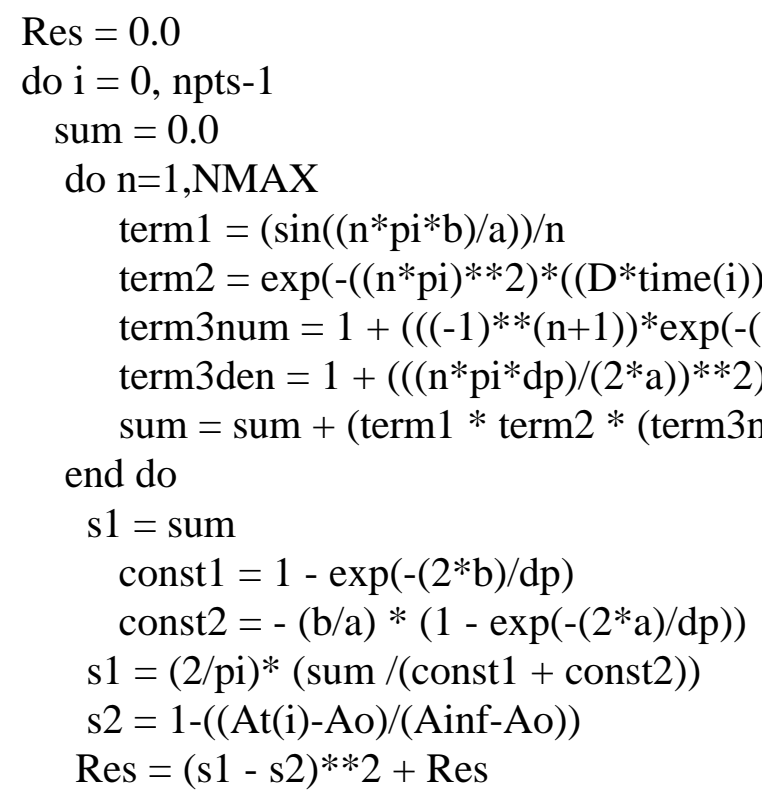


end do

error $=\operatorname{Res} * 1.0 \mathrm{E}+5$

write $\left(6,{ }^{*}\right) \mathrm{X}(1), \mathrm{X}(2)$, error

c $\quad$ write $\left(96,{ }^{*}\right) \mathrm{D}$, Ainf, Res

write $(6, *)$

return

end 


\section{APPENDIX D}

\section{THE FORTRAN PROGRAM “CURVEFIT.FOR"}

Program written with the help of Dr. Sukhtej S. Dhingra

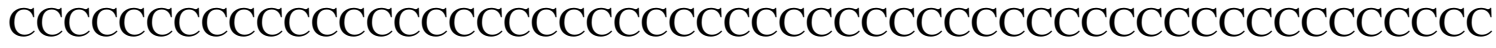
C CURVEFIT.FOR C

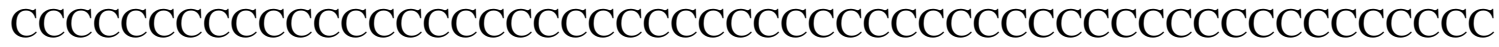

c Program that calculates the value of the absorbance as a function of time

c for the values of D and Ainfinity found by using the program "diff.for"

c $\quad$ Ainf ----> A infinity

c IMAX ----> Max. number of data points

c NMAX ----> max. number (n) for the summation series

program curvefit

parameter $(\mathrm{IMAX}=500, \mathrm{NMAX}=5000)$

real pi, a, b, dp, Ao

real term1, term2, term3num, term3den

real const 1 , const 2

integer npts, $n$

dimension At(0:IMAX), time(0:IMAX), waste(0:IMAX)

dimension Atcal(0:IMAX)

c set the parameters

c $\quad \mathrm{a}, \mathrm{b}$ and dp are in $\mathrm{m}$

$\mathrm{pi}=3.141592654$

$\mathrm{a}=0.0005$

$\mathrm{b}=0.000003$

$\mathrm{dp}=1.2540932 \mathrm{E}-06$

Ao $=0.68329$

c MAKE SURE YOUR DATA FILE IS NAMED "fort.99"

npts $=0$

do $\mathrm{j}=0,500$ 
$\operatorname{read}\left(99,{ }^{*}\right.$, end=101) time(j), waste(j), At(j)

c change the origin for the time

time $(\mathrm{j})=$ time $(\mathrm{j})-40$

npts $=$ npts +1

end do

101 continue

c set the values of D and Ainfinity found by the program "diff.for"

c $\quad \mathrm{D}$ is in $\mathrm{m} 2 . \mathrm{s}-1$

$\mathrm{D}=1.455012 \mathrm{E}-13$

Ainf $=0.3205932$

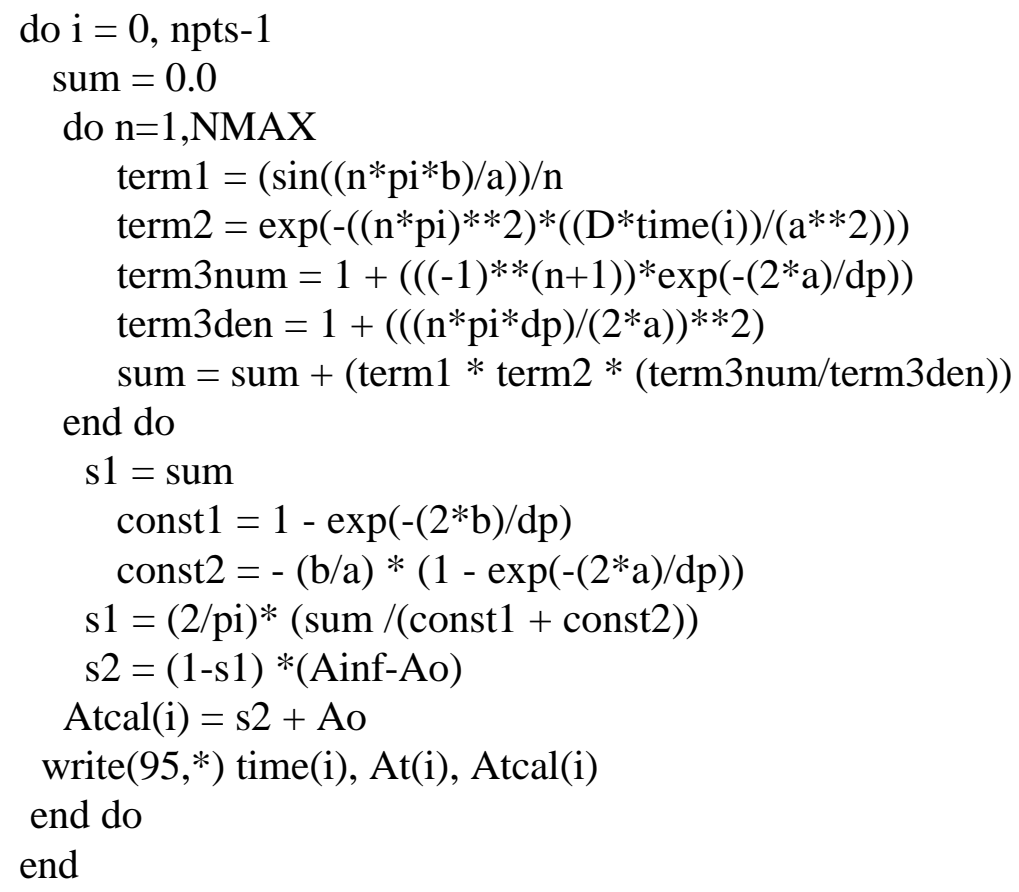




\section{THE FORTRAN PROGRAM “CONC2.FOR”}

СССССССССССССССССССССССССССССССССССССССССССССССССССССC

C CONC2.FOR C

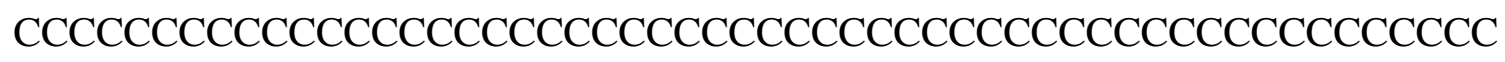

c program to get the concentration changes with time for the PVP at a given distance

c IMAX ----> Max. number of data points

c NMAX ----> max. number (n) for the summation series

program concentration

parameter(IMAX $=500, \mathrm{NMAX}=5000)$

real pi, a, b, D, t, z, C

real term1, term2, term3

integer $n$

c set the parameters

pi $=3.141592654$

c $\quad a$ and $b$ are in $m$

$\mathrm{a}=0.0007$

$\mathrm{b}=0.000003$

c D is the average diffusion coefficient for the PVP

c $\quad \mathrm{D}$ is in $\mathrm{m} 2 . \mathrm{s}-1$

$\mathrm{D}=1.45 \mathrm{E}-12$

c $\quad \mathrm{z}$ is the distance we want to look at

c $\quad z$ is in $m$

$\mathrm{z}=1.3569 \mathrm{E}-06$

c MAKE SURE YOUR DATA FILE IS NAMED "fort.99"

101 continue

c $\quad t$ is the time in $s$ 
do $\mathrm{t}=400,480,10$

c the second time is the time at which the VE appears in the distance of interest $\mathrm{C}=1$

c $\quad \mathrm{C}$ is the normalized concentration of the PVP

c $\quad \mathrm{C}$ in this program is in fact $\mathrm{C}(\mathrm{z}, \mathrm{t}) / \mathrm{Co}$

c before the VE reaches the distance of interest, the normalized concentration is1 write $(95, *)$ t,C

end do

c as soon as the VE reaches the distance of interest, we use the expression derived for

c Fick's law

do $\mathrm{t}=480,1200,10$

$$
\begin{aligned}
\text { sum } & =0.0 \\
\text { do } n & =1, \text { NMAX }
\end{aligned}
$$

term $1=(\sin ((n *$ pi*b $) / a)) / n$

term $2=\cos \left(\left(\mathrm{n}^{*} \mathrm{pi}^{*} \mathrm{z}\right) / \mathrm{a}\right)$

term $3=\exp \left(-(\mathrm{n} * * 2) *(\mathrm{pi} * * 2) * \mathrm{D} *(\mathrm{t}-480) /\left(\mathrm{a}^{* *}\right)\right)$

$\operatorname{sum}=\operatorname{sum}+($ term $1 *$ term $2 *$ term 3$)$

end do

$\mathrm{C}=((\mathrm{b} / \mathrm{a})+((2 / \mathrm{pi}) * \mathrm{sum}))$

write $\left(95,{ }^{*}\right)$ t,C

end do

end 


\section{VITA}

Christelle Marie Laot was born on April 4, 1973, in Provins, France. She grew up in Brittany, region which has been culturally and geographically distinct from the main bulk of the country.

After graduation from high school in 1991 with a Baccalauréat in Mathematics and Physics, she started the five-year engineering program at the Université de Technologie de Compiègne (UTC) in the north-east of Paris. After two years of general engineering, she joined the Department of Chemical Engineering.

While working six months (Fall 1994) for Sicof Inc. on a research project supervised by the European Union, which consisted of perfecting a polymeric coating with semiconductors in order to protect the Acropolis, she was selected to complete her fifth and last year of her undergraduate education as an exchange student in the United States. She participated in the UTC-Virginia Polytechnic Institute and State University exchange program and enrolled in August 1995 in the Master of Science program in Chemical Engineering at Virginia Tech, Blacksburg, Virginia.

In January 1997, after six months of research, she submitted a report to her Grande Ecole and expects to receive soon her Diplôme d'Ingénieur in Génie des Procédés Industriels with emphasis on the fields of quality and environment.

Upon completion of the requirements for a Master of Science degree, Christelle will start working on mixed gas transport through polymeric membranes for her $\mathrm{PhD}$ degree in Chemical Engineering at Virginia Tech.

Christelle Marie Laot 